NBSIR 82-2562

A System for Fire Safety
Evaluation for Multifamily Housing

U.S. DEPARTMENT OF COMMERCE

National Bureau of Standards

National Engineering Laboratory

Center for Fire Research

Washington, DC 20234

September 1982

Interim Report

red for:

Department of Housing and Urban Development lington, DC 20410 



\section{A SYSTEM FOR FIRE SAFETY \\ EVALUATION FOR MULTIFAMILY HOUSING}

H. E. Nelson and A. J. Shibe

U.S. DEPARTMENT OF COMMERCE

National Bureau of Standards

National Engineering Laboratory

Center for Fire Research

Washington, DC 20234

September 1982

Interim Report

Prepared for:

The Department of Housing and Urban Development Washington, DC 20410

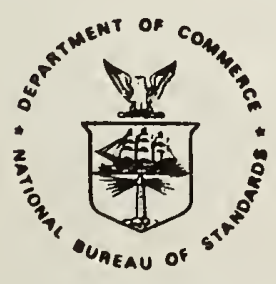

U.S. DEPARTMENT OF COMMERCE, Malcolm Baldrige, Secretary NATIONAL BUREAU OF STANDARDS, Ernest Ambler, Director 



\section{PREFACE}

This report describes a new Fire Safety Evaluation System for Multifamily Housing, developed specifically to identify alternative combinations of widely accepted fire protection systems and building design features that provide a level of fire safety equivalent to that required by the HUD Minimum Property Standards, March 1980. This work is an extension of research conducted by the Center for Fire Research with support from the Department of Health and Human Services towards development of a Fire Safety Evaluation System for Health Care Facilities.

The Design Concepts Research Group, Center for Fire Research, National Engineering Laboratory, National Bureau of Standards, performed the research described in this report under the sponsorship of the Department of Housing and Urban Development. 

PREFACE . . . . . . . . . . . . . . . . . . .

LIST OF FIGURES . . . . . . . . . . . . . . . . . . v v

ABSTRACT . . . . . . . . . . . . . . . . . . . . 1

1. INTRODUCTION . . . . . . . . . . . . . . . . . 1

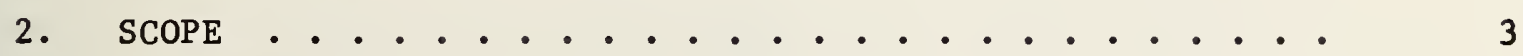

3. PURPOSE . . . . . . . . . . . . . . . . . . . 3

4. EQUIVALENCY CONCEPT . . . . . . . . . . . . . . . 4

5. PROJECT METHODOLOGY . . . . . . . . . . . . . 5

6. SYSTEM DESCRIPTION . . . . . . . . . . . . . . 7

6.1 Capabilities and Limitations . . . . . . . . 7

6.2 Safety Parameters .............. 9

6.3 Safety Parameters Evaluation . . . . . . . . . 11

6.4 Redundant Safety Subsystem . . . . . . . . . . 11

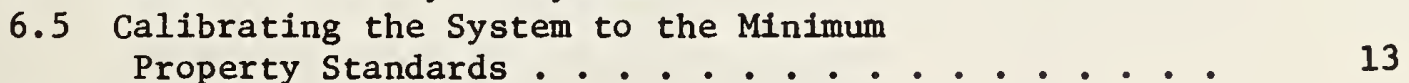

7. COMPUTER ANALYSIS . . . . . . . . . . . . . . 14

8. FIRE SAFETY EVALUATION WORKSHEET FOR

MULTIFAMILY HOUSING . . . . . . . . . . . . . 15

9. SUMMARY . . . . . . . . . . . . . . . 16

10. REFERENCES . . . . . . . . . . . . . . . . 86

APPENDIX A - NBS Delphi Group . . . . . . . . . . . 87

APPENDIX B - Peer Group . . . . . . . . . . . . . . . 9 93

APPENDIX C - Computer Program . . . . . . . . . . . . 97

APPENDIX D - Instructional Manual . . . . . . . . . . 133 



\section{LIST OF FIGURES}

Page

Figure 1. Fire Safety Evaluation Worksheets . . . . . . . . . . 17

Figure 2. Safety Parameters . . . . . . . . . . . . . . . 24

Figure 3. Safety Parameter Matrix . . . . . . . . . . . 25

Figure 4a. MPS Requirements Multifamily Housing $\leq 3$ story $->8$ units floor . . . . . . . . . . . .

Figure 4b. Mandatory Safety Requirements Multifamily Housing $\leq 3$ story - $>8$ units floor . . . . . . . . . . . .

Figure 5a. MPS Requirements Multifamily Housing 4-6 story - > 8 units/floor . . . . . . . . . . .

Figure 5b. Mandatory Safety Requirements Multifamily Housing 4-6 story - > 8 units/floor . . . . . . . . . . . .

Figure 6a. MPS Requirements Multifamily Housing $>6$ story - > 8 units/floor ..............

Figure 6b. Mandatory Safety Requirement Multifamily Housing $>6$ story - > 8 units/floor .............

Figure 7a. MPS Requirements Multifamily Housing $\leq 3$ story $-\leq 8$ units/floor ..............

Figure 7b. Mandatory Safety Requirements Multifamily Housing $\leq 3$ story $-\leq 8$ units/floor . . . . . . . . . . .

Figure 8a. MPS Requirements Multifamily Housing 4-6 story $-\leq 8$ units/floor .............

Figure 8b. Mandatory Safety Requirements Multifamily Housing 4-6 story $-\leq 8$ units/floor.

Figure 9a. MPS Requirements Multifamily Housing $>6$ story $-\leq 8$ units/floor

Figure 9b. Mandatory Safety Requirements Multifamily Housing $>6$ story $-\leq 8$ units/floor . . . . . . . . . . .

Figure 10a. MPS Requirements Housing for the Elderly $\leq 3$ story $->8$ units/floor ...............

Figure 10b. Mandatory Safety Requirements Housing for the Elderly $\leq 3$ story - > 8 units/floor 


\section{LIST OF FIGURES (Continued)}

Page

Figure 11a. MPS Requirements Housing for the Elderly

4-6 story - > 8 units/floor . . . . . . . . . .

Figure 1lb. Mandatory Safety Requirements Housing for the Elderly

4-6 story - > 8 units/floor . . . . . . . . . . .

Figure 12a. MPS Requirements Housing for the Elderly

$>6$ story - > 8 units/floor . . . . . . . . . . .

Figure 12b. Mandatory Safety Requirements Housing for the Elderly

$>6$ story - > 8 units/floor .............

Figure 13a. MPS Requirements Housing for the Elderly

$\leq 3$ story $-\leq 8$ units/floor . . . . . . . . . . .

Figure 13b. Mandatory Safety Requirements Housing for the Elderly $\leq 3$ story $-\leq 8$ units/floor ..............

Figure 14a. MPS Requirements Housing for the Elderly 4-6 story $-\leq 8$ units/floor . . . . . . . . . . .

Figure 14b. Mandatory Safety Requirements Housing for the Elderly 4-6 story $-\leq 8$ units/floor . . . . . . . . . . .

Figure 15a, MPS Requirements Housing for the Elderly $>6$ story $-\leq 8$ units/floor . . . . . . . . . . .

Figure 15b. Mandatory Safety Requirements Housing for the Elderly $>6$ story $-\leq 8$ units/floor . . . . . . . . . . .

Figure 16a. MPS Requirements Rehab. Multifamily Housing $\leq 3$ story - > 8 units/floor . . . . . . . . . . .

Figure 16b. Mandatory Safety Requirements Rehab. Multifamily Housing $\leq 3$ story - $>8$ units/floor . . . . . . . . . . .

Figure 17a. MPS Requirements Rehab. Multifamily Housing 4-6 story - > 8 units/floor . . . . . . . . . . .

Figure 17b. Mandatory Safety Requirements Rehab. Multifamily Housing 4-6 story - > 8 units/floor . . . . . . . . . . .

Figure 18a. MPS Requirements Rehab. Multifamily Housing $>6$ story - > 8 units/floor . . . . . . . . . . .

Figure 18b. Mandatory Safety Requirements Rehab. Multifamily Housing $>6$ story - > 8 units/floor 


\section{LIST OF FIGURES (Continued)}

Page

Figure 19a. MPS Requirements Rehab. Multifamily Housing

1-2 story $-\leq 8$ units/floor . . . . . . . . . . .

Figure 19b. Mandatory Safety Requirements Rehab. Multifamily Housing 1-2 story $-\leq 8$ units/floor . . . . . . . . . . . .

Figure 20a. MPS Requirements Rehab. Multifamily Housing 3 story $-\leq 8$ units/floor . . . . . . . . . . .

Figure 20b. Mandatory Safety Requirements Rehab. Multifamily Housing 3 story $-\leq 8$ units/floor . . . . . . . . . . . .

Figure 21a. MPS Requirements Rehab. Multifamily Housing 4-6 story $-\leq 8$ units/floor . . . . . . . . . . .

Figure 21b. Mandatory Safety Requirements Rehab. Multifamily Housing 4-6 story $-\leq 8$ units/floor . . . . . . . . . . .

Figure 22a. MPS Requirements Rehab. Multifamily Housing

$>6$ story $-\leq 8$ units/floor . . . . . . . . . . .

Figure 22b. Mandatory Safety Requirements Rehab. Multifamily Housing $>6$ story $-\leq 8$ units/floor . . . . . . . . . . .

Figure 23a. MPS Requirements Rehab. Housing for the Elderly $\leq 3$ story - > 8 units/floor . . . . . . . . . . . .

Figure 23b. Mandatory Safety Requirements Rehab. Housing for the Elderly $\leq 3$ story $->8$ units/floor . . . . . . . . .

Figure 24a. MPS Requirements Rehab. Housing for the Elderly 4-6 story $->8$ units/floor ..............

Figure 24b. Mandatory Safety Requirements Rehab. Housing for the Elderly 4-6 story $->8$ units/floor ..........

Figure 25a. MPS Requirements Rehab. Housing for the Elderly $>6$ story $-\leq 8$ units/floor ..............

Figure 25b. Mandatory Safety Requirements Rehab. Housing for the Elderly $>6$ story $-\leq 8$ units/floor ...........

Figure 26a. MPS Requirements Rehab. Housing for the Elderly 1-2 story $-\leq 8$ units/floor . . . . . . . . . . .

Figure 26b. Mandatory Safety Requirements Rehab. Housing for the Elderly 1-2 story $-\leq 8$ units/floor . . . . . . . . 
Figure 27a. MPS Requirements Rehab. Housing for the E1derly 3 story $-\leq 8$ units/floor . . . . . . . . . . .

Figure 27b. Mandatory Safety Requirements Rehab. Housing for the Elderly 3 story $-\leq 8$ units/floor . . . . . . . . .

Figure 28a. MPS Requirements Rehab. Housing for the Elderly 4-6 story $-\leq 8$ units/floor . . . . . . . . . . .

Figure 28b. Mandatory Safety Requirements Rehab. Housing for the Elderly 4-6 story $-\leq 8$ units/floor . . . . . . . .

Figure 29a. MPS Requirements Rehab. Housing for the Elderly $>6$ story - > 8 units/floor .............

Figure 29b. Mandatory Safety Requirements Rehab. Housing for the Elderly > 6 story $->8$ units/floor . . . . . . . .

Figure 30a. MPS Requirements Existing Multifamily and Housing for the Elderly 1-2 story .. . . . . . . . . . . .

Figure 30b. Mandatory Safety Requirements Existing Multifamily and Housing for the Elderly $1-2$ story ............

Figure 31a. MPS Requirements Existing Multifamily and Housing for the Elderly 3 story ..................

Figure 31b. Mandatory Safety Requirements Existing Multifamily and Housing for the Elderly 3 story . . . . . . . . .

Figure 32a. MPS Requirements Existing Multifamily and Housing for the Elderly 4-6 story . . . . . . . . . . . . .

Figure 32b. Mandatory Safety Requirements Existing Multifamily and Housing for the Elderly 4-6 story . . . . . . . . .

Figure 33a. MPS Requirements Existing Multifamily and Housing for the Elderly > 6 story . . . . . . . . . . . . . .

Figure 33b. Mandatory Safety Requirements Existing Multifamily and Housing for the Elderly > 6 story . . . . . . . . . 
A SYSTEM FOR FIRE SAFETY EVALUATION OF MULTIFAMILY HOUSING

An Interim Report

H. E. Nelson and A. J. Shibe

Abstract

A qualitative evaluation system for grading multifamily housing in terms of fire safety has been developed and is ready for testing in Department of Housing and Urban Development field offices. The system is designed to be used to identify various combinations of widely accepted fire safety equipment and building construction features that provide a level of safety equal to or greater than that achieved by strict conformance to the explicit requirements of the HUD Minimum Property Standards. In this evaluation, equivalent safety performance is gauged in terms of overall level of safety provided rather than by a component by component comparison.

\section{INTRODUCTION}

Identifying levels of safety in existing buildings and providing costeffective minimum levels of fire safety in multifamily residences are major concerns of the Department of Housing and Urban Development. Rising construction and operating costs, coupled with more stringent building codes, are forcing the builders of multifamily residences to assess carefully the alternative means by which they may design, construct, or rehabilitate their buildings. 
The National Bureau of Standards, Center for Fire Research, with support from the Department of Health and Human Services (HHS), has previously developed a method for determining how combinations of several widely accepted fire safety practices could be used to provide a level of safety equivalent to that required by the Life Safety Code promulgated by the National Fire Protection Association (NFPA). The method is called the Fire Safety Evaluation System for Health Care Facilities. A detailed description can be found in H. E. Nelson and A. J. Shibe, "A System for Fire Safety Evaluation of Health Care Facilities", NBSIR-78-1555-1 [1]*. HHS has adopted that system in their fire safety requirements for institutions receiving funds for the support of Medicaid and Medicare clients. The NFPA has included the system as part of the 1981 Life Safety Code [2]. The system described in this document is an extension of the concepts used in the health care facility system.

The Department of Housing and Urban Development's (HUD) standard criteria for multifamily residences are stipulated in the "Minimum Property Standards for Multifamily Housing" (MPS) [3]. The MPS identify minimum levels of fire safety in new bulldings designed or built with financial support from HUD. While the MPS prescribe specific approaches to fire safety in new multifamily buildings, they also allow "equivalent" solutions. But, the MPS neither define alternative approaches nor provide a mechanism for evaluating equivalency among the alternatives. For rehabilitation construction and for existing buildings, the MPS do not provide specific fire safety criteria but give general guidance on how to achieve the desired levels of fire safety.

* Numbers in brackets refer to literature references listed at the end of this paper. 
The remainder of this report describes a new Fire Safety Evaluation System (FSES) for Multifamily Housing developed specifically to determine alternative combinations of widely accepted fire safety systems and arrangements that provide a level of safety equivalent to that required by the 1980 MPS. The system is intended to evaluate designs for proposed new buildings, for renovation of existing bulldings, or for existing buildings as they stand.

\section{SCOPE}

This evaluation system applies to multifamily residences only. The total system covers all aspects of bullding fire safety currently covered by the MPS. A few of the items related to built-in utility systems and to roofs and balconies have been excluded from the grading portion of the system. An attachment to the basic evaluation system provides separate consideration for these items. In order to demonstrate equivalent fire safety, the building must both satisfy the requirements in this attachment and equal or excel the minimum point scores in the grading portion of the evaluation system.

\section{PURPOSE}

The purpose of the evaluation system is to provide a rationally based mechanism applicable to multifamily facilities for:

a. Evaluating an existing facility to determine how the actual level of fire safety in that facility compares with the level of fire safety that would be provided by strict conformance to the requirements of the 1980 MPS. 
b. Evaluating various alternative approaches available to upgrade an existing facility to a level of fire safety that meets or exceeds the level prescribed by the MPS.

c. Evaluating a proposed new facility design to determine how its level of fire safety would compare to that required for such a facility by the MPS.

\section{EQUIVALENCY CONCEPT}

The evaluation system provides a method for determining the design features needed to provide equivalence to the 1980 MPS. Equivalency, for the purpose of the system, is judged by comparing the total fire safety level prescribed by the MPS for multifamily facilities to the actual safety level in a particular design or an actual facility.

Equivalency determination is based on the concept that, while the MPS documents do not include a specific statement of the level of safety provided, 1t is possible by examining the MPS requirements to impute one. This imputed level becomes a base line for comparing the level of safety provided by conformance to MPS requirements with the level of safety provided by alternative systems of safeguards. This comparison is made on the basis of the total safety performance of the building, including all of 1 ts safeguards, without making direct comparisons between a specific standard requirement for an element and the corresponding element as it exists in the building. 
Evaluation of equivalency of fire safety involves consideration of two separate concepts:

a. General Fire Safety Requirement - the ability of the building and its fire protection systems to provide measures of general safety commensurate with MPS requirements.

b. Safety Redundancy - in-depth (redundant) protection through the simultaneous provision" of alternative approaches to safety such as fire control, egress, and refuge. Each approach independently provides at least a minimum level of protection. The design of the complete fire safety system incorporating all the alternatives is intended to ensure that the failure of a single protection device or method will not result in a failure of the entire system. This is further discussed in section 6.4 .

\section{PROJECT METHODOLOGY}

Development of the fire safety evaluation system consisted of three operations: system design, professional judgement review and critique, and system testing.

a. System Design. This consisted of: (1) analysis of the stated requirements of the 1980 MPS in terms of the specific functional contribution to fire safety of each MPS requirement, (2) organization of the results of this analysis into a format suitable for soliciting professional judgements of the 
comparative worth of the fundamental requirements relative to the MPS objectives for multifamily facilities, (3) development of a computer program to evaluate alternative designs and fire protection systems, and (4) incorporation of systems changes resulting from the iterative professional judgement review and system tests.

b. Professional Judgement Participation and Review. Professional judgement input was asked and received from two different groups: (1) an NBS group, through the mechanism of a "Delphi" exercise (see Appendix A for description of the NBS Delphi operation), and (2) an outside "Peer" review group (see Appendix B). The Delphi group (an ad hoc group of fire protection experts in the NBS Center for Fire Research) refined the format, established initial values of the safety parameters, and provided judgement values for selecting individual safety parameters in the redundancy evaluations. The outside Peer group consisted of prominent persons in the field of regulation and specification of fire safety requirements in residential facilities. The Peer group provided broad-based technical information and judgemental insight for improving both the format and the final values assigned to the safety parameters and the redundancy factors.

c. System Testing. The testing involved a series of exercises to determine the validity of the fire evaluation system. These exercises included both field tests of actual facilities by NBS 
personnel and computer analysis of hypothetical alternative design systems. (The Department of Housing and Urban Development is planning to further test the system using their engineering staff in the HUD regional offices.)

\section{SYSTEMS DESCRIPTION}

\subsection{Capabilities and Limitations}

The system that has evolved from this effort provides a means of mixing recognized and proven fire safety systems and approaches during facility design and then evaluating these mixes in terms of the overall fire safety performance of the facility. The system permits comparative evaluations of the fire risks and fire safety factors implicit in individual facilities or designs. Those features (parameters) that are in excess of minimum safety requirements are given appropriate credit, reflecting the degree of additional safety provided. This is in contrast to strict adherence to MPS where no credit is given for meeting higher safety levels than the minimum for a given feature. The credit, however, is limited in its application to those areas of fire safety impact where the safeguards provide credible improvements in safety. Conversely, features that increase one or more aspects of fire risk are appropriately charged for their detrimental impact on safety. The FSES solution is intended to be an assessment of total safety performance as compared to measuring compliance with a minimum safety level. The result provides opportunity for cost reduction, wider choice of design alternatives, and operational flexibility at greater levels than currently available through strict, literal compliance with the MPS requirements. 
The Fire Safety Evaluation Worksheet (FSES) is the instrument used to determine equivalency and is illustrated in Figure 1. The worksheet in Figure 1 is supported by a glossary, included as Appendix $D$ in this report.

The most important limitations of the evaluation system are:

a. As presently developed the evaluation system applies only to the 1980 MPS, although it could readily be revised to meet changes in the MPS.

b. The results are expressed in equivalency to the level of safety achieved by the MPS, and should not be construed as a measure of total or absolute fire safety.

c. The system, like all existing methods for regulating or evaluating fire safety, is only partially supported by technical information or statistics. The professional judgement of experts in a series of balanced peer-consensus groups is used to bridge the gaps in reproducible, reliable technical knowledge.

d. In general the evaluation system is limited to evaluating the interrelationships of those fire safety methodologies and approaches that are defined in the MPS. There is no basis in the system for accommodating completely innovative approaches (such as automatic venting at the point of fire or the use of halogenated gases as a general protection system). 
e. While the parameter measurements of the system cover built-in structural materials and elements, space arrangement, and fire protection systems and devices, the system does not provide alternate approaches to meeting the MPS requirements for:

(1) Utilities such as heating, air conditioning, electrical, and incinerator systems.

(2) Balcony and Roof Requirements.

(3) Administrative activities such as emergency plans and fire drills.

When using the evaluation system, the additional fire safety requirements in these areas are applied in the traditional manner of explicit conformance with the established standards and requirements as described and/or referenced in the MPS.

\subsection{Safety Parameters}

The safety parameters are measures of those building and fire protection features that bear upon the safety of residents (and other occupants) who may be in the building at the time of a fire. 
The safety parameters were selected by examining the specific element requirements of the 1980 MPS, and by evaluating the contribution of various elements of the standard to safety. The selected safety parameters were modified first by the NBS Delphi panel and later by the Peer group. The selected safety parameters are shown in Figure 2, Safety Parameters.

Each of the safety parameters was analyzed. Where the current standard requirements recognize several levels of a parameter (e.g॰, the MPS recognizes seven different levels of construction), all have been included in the matrix. In addition, conditions likely to be encountered in situations failing to meet the explicit requirements, and conditions exceeding those required by the code but available for increased protection were also listed. Figure 3 shows the final "matrix" form of the 14 selected safety parameters, each having three to eight subdivisions.

The safety parameters are designed to constitute a complete assembly of those basic building features for which equivalency can be expressed in determining the level of safety in a multifamily facility. An additional series of items required by the MPS but outside the scope of the equivalency covered by the listed safety parameters relate primarily to building utilities, operational procedures and areas per floor (see discussion in section 6.1.e). A companion check list covering these items has been prepared as part of the evaluation system worksheets. This check list is included in Figure 1 as Subfigure $1 \mathrm{f}$. 


\subsection{Safety Parameter Evaluation}

A Delphi type group was established to bring the most informed judgement and experience together to assess the relative impact on general safety of each of the parameters in each of the potential conditions listed.

Each member of the group was provided with copies of an initial matrix similar to the one shown in Figure 3. Each person then evaluated the relative importance with respect to fire safety of each item in the entire matrix of parameters without consultation with other members of the group. The members of the Delphi group were advised that the risk being considered covered new and existing multifamily facilities and that the objective was a system to measure equivalency with the safety level provided by the 1980 MPS. The value judgements made by this group are, therefore, considered to be based on their understanding of the character and needs of residents in multifamily facilities.

\subsection{Redundant Safety Subsystems}

A basic principle of the MPS is that there will be a redundancy of protection so that the failure of a single protection device or method will not result in failure of the entire safety system. In addition, the development of a redundant approach, as used in this safety evaluation system, avoids the pitfall of traditional approaches sometimes used in grading systems where al1 of the elements are considered mutually exclusive of each other and a single total score determines acceptability. It is possible under such a system to fail to detect the absence of a critical element. The evaluation 
system establishes redundancy on the basis of 1n-depth coverage of principal fire safety methodologies. The redundant methodologies used in the system are those related to fire safety through fire control, egress, and refuge.

The redundant methodologies were chosen after examination of decision tree approaches $[4,5]$. These approaches divide fire protection into elements. Those elements related to "Manage Fire" (1.e, the control of fuel and arrangement, compartmentation, and other mechanisms of containment of fire and its impacts, extinguishment suppressions and other means of terminating fire development) are incorporated into Fire Control.

"Manage Exposed" (i.e., the provision of safe location of refuge either by evacuation or by establishment of safe areas of refuge) was subdivided into two redundancy methodologies, egress and refuge.

Each member of the Delphi groups identified the Importance of each safety parameter relative to the separate fire safety methodologies of fire control, egress, and refuge. These group results were processed and analyzed by project staff at NBS and then reviewed in subsequent conference meetings of the Delphi group. By this process, the parameters that have a significant impact on each of the redundant methodologies were identified. It was found that many of the parameters affect more than one of the methodologies. In the judgement of the group, sprinklers, separations of living units, vertical openings, and protection of hazardous areas impact on all three. Figure Ic shows the breakdown in terms of which parameters apply to which methodologies. 


\subsection{Calfbrating the System to the Minimum Property Standards}

The system as developed can be used to obtain scores for four individual safety factors. These factors are entitled general fire safety, fire control, egress provided, and refuge provided. A minimum score defining equivalency to the MPS for each of the four safety factors is required as the base of reference for determining equivalency by this system. This section describes how these minimum values were set.

The MPS provides explicit requirements for New Multifamily Housing and for New Housing for the Elderly. The MPS, however, provides only general guidance on how to achieve the desired fire safety levels for Rehabilitation Multifamily Housing, Rehabilitation Housing for the Elderly, Existing Multifamily Housing and Existing Housing for the Elderly. HUD officials who were also members of the Peer group, translated the general guidelines into specific requirements matching the parameters in the FSES.

For each set of specific MPS requirements, there is a corresponding set of requirements in Table 1 (Figure $1 \mathrm{~b}$ ) that corresponds to each of the 14 safety parameters. For example, Figure $4 \mathrm{a}$ shows the requirements for Multifamily Housing with three or fewer stories and more than 8 units per floor: a circle is placed in each box that corresponds to the requirements. The mininmum values are determined by using Table 2 (Figure 1c). The circled values in Table 1 (see Figure 4a) are transferred to the appropriate unshaded blocks in Table 2 (Figure 1c). Where the block contains a $\div 2$, one half of the value shown in Figure $1 \mathrm{~b}$ is entered. The four columns are each summed. For example, the requirements in Figure $4 \mathrm{a}$ are transferred to Figure $4 \mathrm{~b}$ and 
the minimum score for fire control is 5.5 , for egress provided is 8 , for refuge provided is 8 , and for general fire safety is 7 . These minimum safety requirements define the minimum scores for equivalence in multifamily housing with three or fewer stories, and more than 8 units per floor.

Fire safety requirements for each set of requirements with subdivisions for building height and number of living units were determined using this approach. The resulting values are the basis of equivalency for each area of requirement. The final resultant values constitute the Mandatory Safety Requirements shown in Figures $1 d-1$ and $1 d-2$. The specific parameter values and the results are shown in Figures 4 through 33. Equivalency for any specific facility requires that the scores for the evaluated facility in each of the four individual safety factors (i.e., General Fire Safety, Fire Control, Egress Provided, and Refuge Provided) equal or exceed score achleved for the set of MPS requirements that apply to that specific type and class of facility.

\section{COMPUTER ANALYSIS}

The evaluation system is theoretically capable of evaluating each of about 3 billion combinations of the 14 safety parameters and variations (but practical arrangements in existing buildings and future designs are of the order of a few thousand). A computer program, described in Appendix C, was developed for verifying the original proposed system as well as to analyze potential proposed changes. The program can generate all alternative combinations of parameter values (1.e., safety features) that the FSES will indicate as equivalent to the MPS. 
By using the computer program, the evaluator can review all acceptable solutions for a given bullding configuration and can also be assured that answers are not blased by individual preference. The printouts of the building arrangements can be easily analyzed by an experienced individual to establish acceptability of solutions. The computer generated building configurations, based on the FSES guidelines, were used by the NBS staff and the consultant group to compare MPS levels of safety to the FSES proposed levels of safety.

\section{FIRE SAFETY EVALUATION WORKSHEET FOR MULTIFAMILY HOUSING}

All of the parameters, varlables, and formulas for determining the facility safety equivalency with the MPS are contained in a self-instructing form. A separate manual (Appendix D) was prepared to assist in completion of the evaluation form. The manual provides expanded discussions and definitions of various items in the evaluation sheet to assist the surveyor or reviewer when questions of definition, interpretations, or meaning arise.

HUD particlpants in this project suggested including an additional safety parameter level for Parameter 9, "Separation of Living Units from Each Other and from Common Spaces". This spectal level covers a configuration defined as wall separations having a two-hour or greater fire resistance with any doors in such walls being class "C" or better (fire doors having a fire resistance rating of 45 minutes). It is intended that the safety parameter value for this additional parameter level is to be awarded only where the building construction is Type 1 or Type 2a. A preliminary computer analysis shows that parameter levels between 6 and 10 produce bullding configurations allowing a 
range of types of alternative systems not otherwise available. The proposed HUD field tests may also produce a number of building configurations which will include this type of superior separation. Based on the results of HUD field tests and with additional computer analysis, NBS will present to the peer group safety values for the superior separation environment for their appraisal. After that group has made their appraisal for the appropriate action on inclusion and safety value, NBS will recommend the necessary changes in Table 1, "Safety Parameter Values".

\section{SUMMARY}

The conclusions resulting from this study are briefly stated as follows:

a. A method has been developed and described for generating equivalency to a specified set of occupancy safety requirements. This method is based on the articulation of levels of building safety and on the redundancy of safeguards. This method can provide the necessary flexibility enabling a designer to achieve minimum cost solutions for a specified level of safety.

b. The described method "System for Fire Safety Evaluation of Multifamily Housing" is a specific example of an equivalency approach. The system provides equivalency to the minimum life safety requirements for the 1980 MPS and can be revised to reflect later editions of the MPS. 


\section{FIRE SAFETY EVALUATION WORKSHEET}

FOR MULTIFAMILY RESIDENCES

BUILDING IDENTIFICATION

EVALUATOR DATE

Complete one worksheet for each building evaluated.

STEP 1. Complete the building identification, evaluator and date entries above.

Figure 1a. Fire Safety Evaluation Worksheet 
Step 2. Determine Sately Parameter Values - Table 1.

A. Seiect and circie the parameter value for each safely parameter in Table 1 that best describes the conditions in the building. Choose oniy one value for each of the 14 satety paramelers. If two or more appear to appiy, choose the one with the lowest point value.

\section{Table 1. SAFETY PARAMETER VALUES}

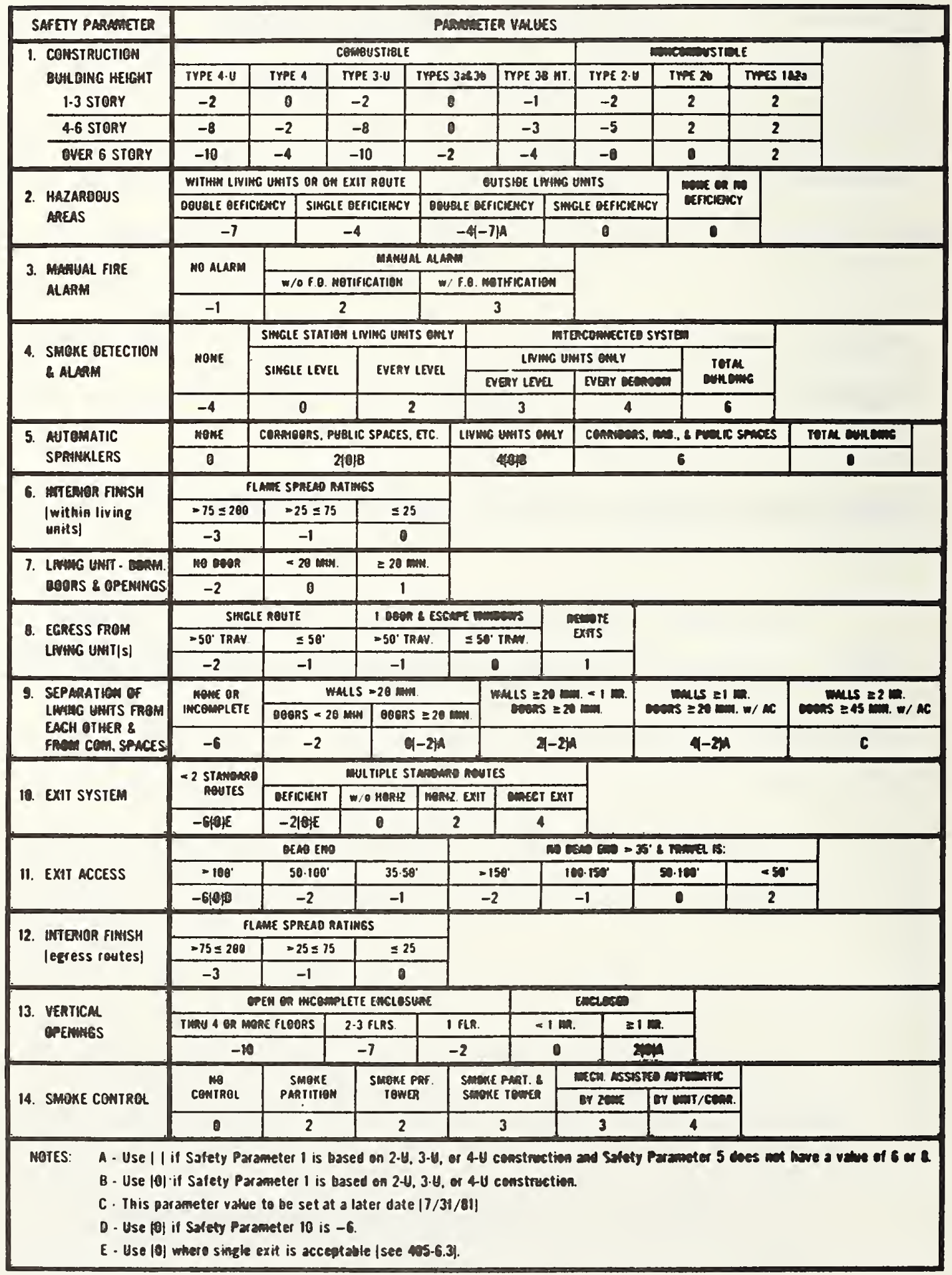

Figure 1b. Fire Safety Evaluation Worksheet 
STEP 3. COMPUTE INDIVIDUAL SAFETY EVALUATIONS - USE TABLE 2

1. Transfer each of the 14 circled safety parameter values on Table 1 to every unshaded block in the line with the corresponding safety parameter in Table 2. Where the block is indicated $(\div 2)$ enter only one-half the value shown in Table 1.

2. Add the four columns, keeping in mind that any negative numbers deduct.

3. Transfer the resulting values for $S_{1}, S_{2}, S_{3}$ and $S_{4}$ to table on page 3 of this worksheet.

\section{Table 2. INDIVIDUAL SAFETY EVALUATIONS}

\begin{tabular}{|c|c|c|c|c|}
\hline SAFETY PARAWETER & $\begin{array}{l}\text { FHeE } \\
\text { COATREL } \\
\left(S_{1} \mid\right.\end{array}$ & 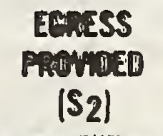 & 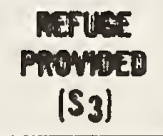 & $\begin{array}{l}\text { CEMERAL FIRE } \\
\text { SAFETY PROHDED } \\
\text { (S4) }\end{array}$ \\
\hline \multicolumn{5}{|l|}{ 1. CONSTRUCTIAH } \\
\hline 2. HAZARPDQUS AREAS & & $\div 2$ & & \\
\hline 3. MAMUAL FIRE MLAMA & $\div 2$ & & & \\
\hline $\begin{array}{l}\text { 4. SHOKE DETECTIOA \& } \\
\text { ALARH }\end{array}$ & $\div 2$ & & & \\
\hline 5. AUTOAATIC SPRWHLES & & $\div 20,3$ & $(\div 2) A$ & \\
\hline 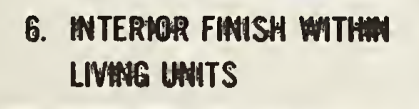 & $\div 2$ & & & \\
\hline \multicolumn{5}{|l|}{$\begin{array}{l}\text { 7. LIVHE UNIT - BORH } \\
\text { DOORS \& OPENHWSS }\end{array}$} \\
\hline \multicolumn{5}{|l|}{$\begin{array}{l}\text { 8. EGRESS FRON LIVING } \\
\text { UNTIS) }\end{array}$} \\
\hline $\begin{array}{l}\text { 9. SEPARATION OF LIVHA } \\
\text { UANTS FROM EACH OTHER } \\
\text { \& FROA COAMON SPACES }\end{array}$ & & $\div 2$ & & \\
\hline 10. EXIT SYSTEM & & & $\div 2$ & \\
\hline \multicolumn{5}{|l|}{ 11. EXIT ACCESS } \\
\hline \multicolumn{5}{|l|}{$\begin{array}{l}\text { 12. INTERIOR FINASH } \\
\text { (egress routes) }\end{array}$} \\
\hline 13. VERTICAL BPEMAGS & $\div 2$ & & & \\
\hline \multicolumn{5}{|l|}{ 14. SMOKE CONTROL } \\
\hline TOTAL & $S_{1}=$ & $\mathbf{s}_{2}=$ & $s_{3}=$ & $S_{4}=$ \\
\hline \multicolumn{5}{|c|}{ 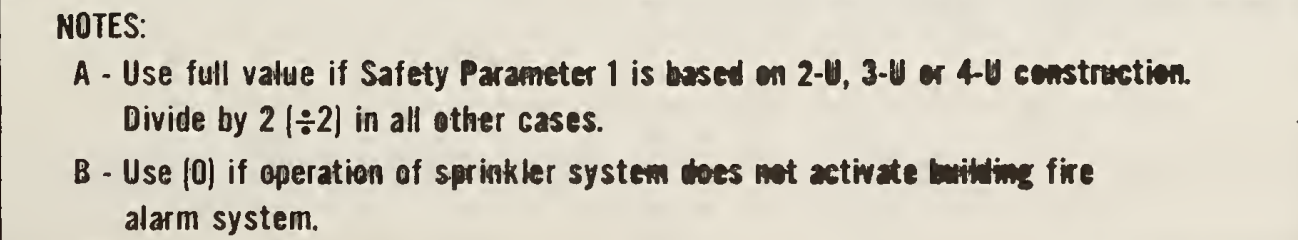 } \\
\hline
\end{tabular}

Figure 1c. Fire Safety Evaluation Worksheet 
STEP 4. DETERMINE MANDATORY SAFETY REQUIREMENT VALUES - USE TABLE 3

A. Using the classification of the building (i.e., New, Rehabilitation or Existing) and the building height, circle the appropriate value in each of the four columns in Table 3 .

B. Transfer the circled values for $S_{a}, S_{b}, S_{c}$, and $S_{d}$ to the blanks marked $S_{a}, S_{b}, S_{c}$, and $S_{d}$ in Table 4.

\section{Table 3A. MANDATORY SAFETY REQUIREMENTS}

\section{(multi-family housing)}

\begin{tabular}{|c|c|c|c|c|c|c|c|c|}
\hline \multirow{2}{*}{$\begin{array}{l}\text { BUHLOW'S } \\
\text { HEIGHT }\end{array}$} & \multicolumn{2}{|c|}{$\begin{array}{l}\text { CEMTROA } \\
\text { REQUANEMEMT } \\
\left(S_{\mathrm{a}}\right)\end{array}$} & \multicolumn{2}{|c|}{ 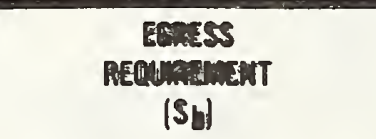 } & \multicolumn{2}{|c|}{ 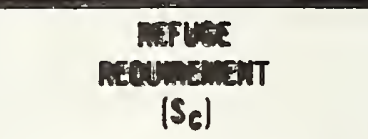 } & \multicolumn{2}{|c|}{ 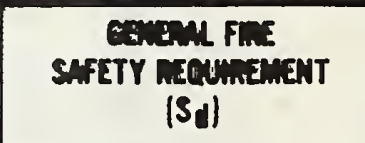 } \\
\hline & $\begin{array}{c}>8 \\
\text { UNIS/FLOQR }\end{array}$ & $\begin{array}{c}\leq 8 \\
\text { UntS/FLon }\end{array}$ & $\begin{array}{c}>8 \\
\text { UATIS/FLOER }\end{array}$ & $\begin{array}{c}\leq 8 \\
\text { undS/FLeen }\end{array}$ & $\begin{array}{c}>8 \\
\text { units/Flem }\end{array}$ & $\begin{array}{c}\leq 8 \\
\text { unts/Flean }\end{array}$ & $\begin{array}{c}>8 \\
\text { unTs/FLcen }\end{array}$ & $\begin{array}{c}\leq 8 \\
\text { UNTS/FLOOR }\end{array}$ \\
\hline$\leq 3$ STORY & 5.5 & 5.5 & 8 & 6 & 8 & 6 & 7 & 5 \\
\hline 4-6 STORY & 8 & 8 & 10 & 8 & 9 & 7 & 10 & 8 \\
\hline$>6$ STORY & 10 & 10 & 10 & 8 & 11 & 9 & 12 & 10 \\
\hline
\end{tabular}

Table 3B. MANDATORY SAFETY REQUIREMENTS (housing for the elderly)

\begin{tabular}{|c|c|c|c|c|c|c|c|c|}
\hline \multirow{2}{*}{$\begin{array}{l}\text { BUHLDHG } \\
\text { HEIGHT }\end{array}$} & \multicolumn{2}{|c|}{$\begin{array}{l}\text { COATROL } \\
\text { REQUHREATEAT } \\
\text { (Sal }\end{array}$} & \multicolumn{2}{|c|}{ 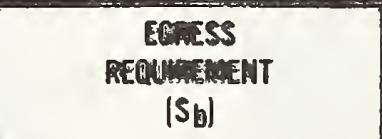 } & \multicolumn{2}{|c|}{ 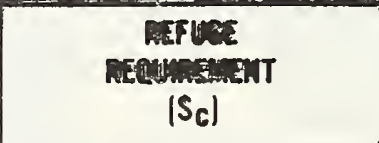 } & \multicolumn{2}{|c|}{ 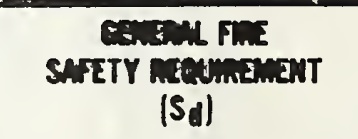 } \\
\hline & $\begin{array}{c}>8 \\
\text { UTS/FLOOR }\end{array}$ & $\begin{array}{c}\leq 8 \\
\text { UAUTS/FLCER }\end{array}$ & $\begin{array}{c}>8 \\
\text { UUTS/FLOBR }\end{array}$ & $\begin{array}{c}\leq 8 \\
\text { uwTS/FL }\end{array}$ & $\begin{array}{c}>8 \\
\text { UTIS/FLen }\end{array}$ & $\begin{array}{c}\leq 8 \\
\text { ums/TLeen }\end{array}$ & $\begin{array}{c}>8 \\
\text { murs/rten. }\end{array}$ & $\begin{array}{c}\leq 8 \\
\text { uns/floan }\end{array}$ \\
\hline$\leq 3$ STERY & 6.5 & 8.5 & 8 & 6 & 8 & 6 & 9 & 7 \\
\hline 4-6 STORY & 9 & 9 & 10 & 8 & 9 & 7 & 12 & 10 \\
\hline >6 STORY & 11 & 11 & 10 & 8 & 11 & 9 & 14 & 12 \\
\hline
\end{tabular}

Figure 1d-1. Fire Safety Evaluation Worksheet 


\section{Table 3C. MANDATORY SAFETY REQUIREMENTS (REHABILITATION MULTIFAMILY HOUSING)}

\begin{tabular}{|c|c|c|c|c|c|c|c|c|}
\hline \multirow{2}{*}{$\begin{array}{c}\text { BUILDING } \\
\text { HEIGHT }\end{array}$} & \multicolumn{2}{|c|}{$\begin{array}{c}\text { CONTROL } \\
\text { REQUIREMENT } \\
\left(S_{a}\right)\end{array}$} & \multicolumn{2}{|c|}{$\begin{array}{c}\text { EGRESS } \\
\text { REQUIREMENT } \\
\left(S_{b}\right)\end{array}$} & \multicolumn{2}{|c|}{$\begin{array}{c}\text { REFUGE } \\
\text { REQUIREMENT } \\
\left(S_{c}\right)\end{array}$} & \multicolumn{2}{|c|}{$\begin{array}{c}\text { GENERAL FIRE } \\
\text { SAFETY REQUIREMENT } \\
\left(S_{d}\right)\end{array}$} \\
\hline & $\begin{array}{c}>8 \\
\text { UNITS/FLOOR }\end{array}$ & $\begin{array}{c}\leq 8 \\
\text { UNTS/FLOOR }\end{array}$ & $\begin{array}{c}>8 \\
\text { UNITS/FLORR }\end{array}$ & \begin{tabular}{c|}
$\leq 8$ \\
UNITS/FLOOR
\end{tabular} & $\begin{array}{c}8 \\
\text { UNITS/FLOOR }\end{array}$ & $\begin{array}{c}\leq 8 \\
\text { UNITS/FLOOR }\end{array}$ & $\begin{array}{c}>8 \\
\text { UNTS/FLOOR }\end{array}$ & $\begin{array}{c}\leq 8 \\
\text { UNITS/FLOOR }\end{array}$ \\
\hline 1-2 STORY & 5.5 & -5 & 5 & -3 & 7 & -4 & 4 & -9 \\
\hline 3 STORY & 5.5 & 5.5 & 5 & 3 & 7 & 5 & 4 & 2 \\
\hline 4-6 STORY & 8 & 6 & 7 & 4 & 8 & 5 & 7 & 3 \\
\hline$>6$ STORY & 10 & 8 & 7 & 4 & 10 & 7 & 9 & 5 \\
\hline
\end{tabular}

\section{Table 3D. MANDATORY SAFETY REQUIREMENTS (REHABILITATION ELDERLY HOUSING)}

\begin{tabular}{|c|c|c|c|c|c|c|c|c|}
\hline \multirow{2}{*}{$\begin{array}{l}\text { BUILDING } \\
\text { HEIGHT }\end{array}$} & \multicolumn{2}{|c|}{$\begin{array}{l}\text { CONTROL } \\
\text { REQUIREMENT } \\
\left(S_{a}\right)\end{array}$} & \multicolumn{2}{|c|}{$\begin{array}{c}\text { EGRESS } \\
\text { REQUIREMENT } \\
\left(S_{b}\right)\end{array}$} & \multicolumn{2}{|c|}{$\begin{array}{c}\text { REFUGE } \\
\text { REQUIREMENT } \\
\text { (Sc) }\end{array}$} & \multicolumn{2}{|c|}{$\begin{array}{c}\text { GENERAL FIRE } \\
\text { SAFETY REQUIREMENT } \\
\left(S_{d}\right)\end{array}$} \\
\hline & $\begin{array}{c}>8 \\
\text { UNTS/FLOOR }\end{array}$ & $\begin{array}{c}\leq 8 \\
\text { UNITS/FLOOR }\end{array}$ & $\begin{array}{c}>8 \\
\text { UNIS/FLOOR }\end{array}$ & $\begin{array}{c}\leq 8 \\
\text { UNTS/FLOOR }\end{array}$ & $\begin{array}{c}>8 \\
\text { UNTS/FLOOF }\end{array}$ & $\begin{array}{c}\leq 8 \\
\text { UNTS/ROOP }\end{array}$ & $\begin{array}{c}>8 \\
\text { UNITS/FLOOR }\end{array}$ & $\begin{array}{c}\leq 8 \\
\text { UNTS/FLOOR }\end{array}$ \\
\hline 1-2 STORY & 6.5 & -4 & 5 & -3 & 7 & -4 & 6 & -7 \\
\hline 3 STORY & 6.5 & 6.5 & 5 & -3 & 7 & -4 & 6 & 4 \\
\hline 4-6 STORY & 9 & 7 & 7 & 4 & 8 & 5 & 9 & 5 \\
\hline$>6$ STORY & 11 & 9 & 7 & 4 & 10 & 7 & $\pi$ & 7 \\
\hline
\end{tabular}

\section{Table 3E. MANDATORY SAFETY REQUIREMENTS (EXISTING MULTIFAMILY \& ELDERLY HOUSING)}

\begin{tabular}{|c|c|c|c|c|c|c|c|c|}
\hline \multirow{2}{*}{$\begin{array}{c}\text { BUILDING } \\
\text { HEIGHT }\end{array}$} & \multicolumn{2}{|c|}{$\begin{array}{l}\text { CONTROL } \\
\text { REQUIREMENT } \\
\left(S_{a}\right)\end{array}$} & \multicolumn{2}{|c|}{$\begin{array}{c}\text { EGRESS } \\
\text { REQUIREMENT } \\
\left(S_{b}\right)\end{array}$} & \multicolumn{2}{|c|}{$\begin{array}{l}\text { REFUGE } \\
\text { REQUIREMENT } \\
\left(S_{c}\right)\end{array}$} & \multicolumn{2}{|c|}{$\begin{array}{l}\text { GENERAL FIRE } \\
\text { SAFETY REQUIREMENT } \\
\left(S_{d}\right)\end{array}$} \\
\hline & $\begin{array}{c}>8 \\
\text { UNITS/ROOR }\end{array}$ & $\begin{array}{c}\leq 8 \\
\text { UNTS/FLOOR }\end{array}$ & $\begin{array}{c}>8 \\
\text { UNTS/FLOOR }\end{array}$ & $\begin{array}{c}\leq 8 \\
\text { UNTS/FLOR }\end{array}$ & $\begin{array}{c}>8 \\
\text { UNTS/FLOR }\end{array}$ & $\begin{array}{c}\leq 8 \\
\text { UNIS/FDOR }\end{array}$ & $\begin{array}{c}>8 \\
\text { UNITS/ROOR }\end{array}$ & $\begin{array}{c}\leq 8 \\
\text { UNISS/FOOR }\end{array}$ \\
\hline 1-2 STORY & -5 & -5 & -5 & -5 & -5 & -5 & -11 & -11 \\
\hline 3 STORY & 4 & 4 & 0 & 0 & 5 & 5 & -1 & -1 \\
\hline 4-6 STORY & 6.5 & 6.5 & 3 & 3 & 5 & $5^{\prime}$ & 2 & 2 \\
\hline$>6 \mathrm{STORY}$ & 7.5 & 7.5 & 3 & 3 & 7 & 7 & 4 & 4 \\
\hline
\end{tabular}


STEP 5 FIRE SAFETY EQUIVALENCY EVALUATION

A. Perform the indicated subtractions in Table 4. Enter the differences in the appropriate answer blocks.

B. For each row check "YES" if the value in the answer block is zero or greater. Check "NO" if the value in tne answer block is a negative number.

\section{Table 4. FIRE SAFETY EQUIVALENCY EVALUATION}

\begin{tabular}{|c|c|c|c|c|c|}
\hline $\begin{array}{l}\text { CONTROL } \\
\text { PROYHDED }\end{array}$ & $\left|S_{1}\right|$ & less & $\begin{array}{l}\text { MEQURPED } \\
\text { CONTROL }\end{array}\left|S_{\mathrm{a}}\right|$ & $\geq 0$ & $\begin{array}{l}s_{1} \\
\square-\square=\square\end{array}$ \\
\hline $\begin{array}{l}\text { EGRESS } \\
\text { PROHIDED }\end{array}$ & $\mid s_{2}$ & less & $\begin{array}{l}\text { REQUMVED } \\
\left.\text { EGRESS } \mid S_{b}\right)\end{array}$ & $\geq 0$ & $\square^{S_{2}}-\square^{S_{b}}={ }^{D}$ \\
\hline $\begin{array}{l}\text { REFUGE } \\
\text { PROVIDED }\end{array}$ & $I_{3}$ & less & $\begin{array}{l}\text { REQUURED } \\
\text { REFUGE }\left(S_{C}\right)\end{array}$ & $\geq 0$ & ${ }^{s_{3}}-S^{s_{c}}-{ }^{R}=\square^{R}$ \\
\hline $\begin{array}{l}\text { GENERAL } \\
\text { FIME } \\
\text { SAFETY }\end{array}$ & $1 S_{4}$ & less & $\begin{array}{l}\text { REQUIRED } \\
\text { GEM. FRRE }\left(S_{d d}\right) \\
\text { SAFETY }\end{array}$ & $\geq 0$ & ${ }^{S_{4}}-{ }^{S_{\mathrm{d}}}=$ \\
\hline
\end{tabular}

CONCLUSIONS :

1. [ ] A1] of the checks in Table 4 are in the "YES" coiumn. The level of fire safety is at least equivalent to that prescribed by the "'inimum Property Standards .*

2. [ ] One or more of the checks in Table 4 are in the "No" column. The level of fire safety is not shown by this system to be equivalent to that prescribed by the Minimum Property Standards.

* The equivalency covered by this worksheet includes the majority of considerations covered by the Minimum Property Standards. There are a few considerations that are not evaluated by this method. These must be considered separately. These additional considerations are covered in Table 5 .

This worksheet has been prepared by the Fire Safety Engineering Division, Center for Fire liesearch, NBS. 
(The following items are required by the MPS as fire safety features but are beyond the scope of equivalency evaluation of the Fire Safety Evaluation System. They must be accounted for separately.)

\begin{tabular}{|c|c|c|c|}
\hline & REQUIREMENTS & YES & No \\
\hline 1. & $\begin{array}{l}\text { Access to the Roof }(405-6.4) \text {. } \\
\text { In buildings } 3 \text { or more stories in height, having slopes < } 20 \text { degrees, } \\
\text { is adequate access provided? }\end{array}$ & & \\
\hline 2. & $\begin{array}{l}\text { Balconies and Porches }(405-11) \text {. } \\
\text { Are balconies and porches non-combustible or meeting the limitations } \\
\text { for combustible installations? }\end{array}$ & & \\
\hline 3. & $\begin{array}{l}\text { Roof Covering }(405-12) \text {. } \\
\text { Does roof covering meet the classification requirements based on type } \\
\text { of construction and building separation? }\end{array}$ & & \\
\hline 4. & $\begin{array}{l}\text { Standpipes }(405-15) \text {. } \\
\text { Do buildings } 5 \text { stories or greater in height have class I service } \\
\text { standpipes meeting NFPA No. } 14 \text { ? }\end{array}$ & & \\
\hline 5. & $\begin{array}{l}\text { Mechanical System ( } 515 \& 615) \text {. } \\
\text { Do mechanical systems (ventilation, heating, cooling, plumbing, water } \\
\text { heating and gas piping) meet the requirement and referenced standards? }\end{array}$ & & \\
\hline 6. & $\begin{array}{l}\text { Electrical ( } 516 \& 616) \text {. } \\
\text { Does the electrical system meet the National Electrical Code? }\end{array}$ & & \\
\hline 7. & $\begin{array}{l}\text { Elevator Capture }(614-2.1) \text {. } \\
\text { Are elevators equipped with emergency capture meeting the ANSI } \\
\text { elevator Code? }\end{array}$ & & \\
\hline 8. & Areas per floors meet the limitations set in Table 4-5.2. & & \\
\hline 9. & $\begin{array}{l}\text { In buildings requiring elevators }(402-9.3) \text {, there is either one } \\
\text { elevator in each smoke compartment or the elevator(s) are accessed } \\
\text { through a lobby separated from each smoke compartment by } 1-\mathrm{hr} \text {. wall } \\
\text { with } 3 / 4-\mathrm{hr} \text {. doors per } 402-9.2 \text {. }\end{array}$ & & \\
\hline 10. & Incinerators and trash chutes conform with NFPA 82 . & & \\
\hline 11. & $\begin{array}{l}\text { The distance of travel to an exit stairway or exterior door from } \\
\text { any point within a boller room or a doorway to an exit corridor } \\
\text { does not exceed } 50 \text { feet. }\end{array}$ & & \\
\hline
\end{tabular}

Figure 1f. Fire Safety Evaluation Worksheet 


\begin{tabular}{|l|}
\hline SAFETY PARAMETER \\
\hline $\begin{array}{l}\text { 1. CONSTRUCIION } \\
\text { BUILDING HEIGHT } \\
\text { 1-3 STORY }\end{array}$ \\
\hline 46 STORY \\
\hline OVER 6 STORY \\
\hline 2. HAZARDOUS \\
AREAS
\end{tabular}

Figure 2. Safety Parameters 


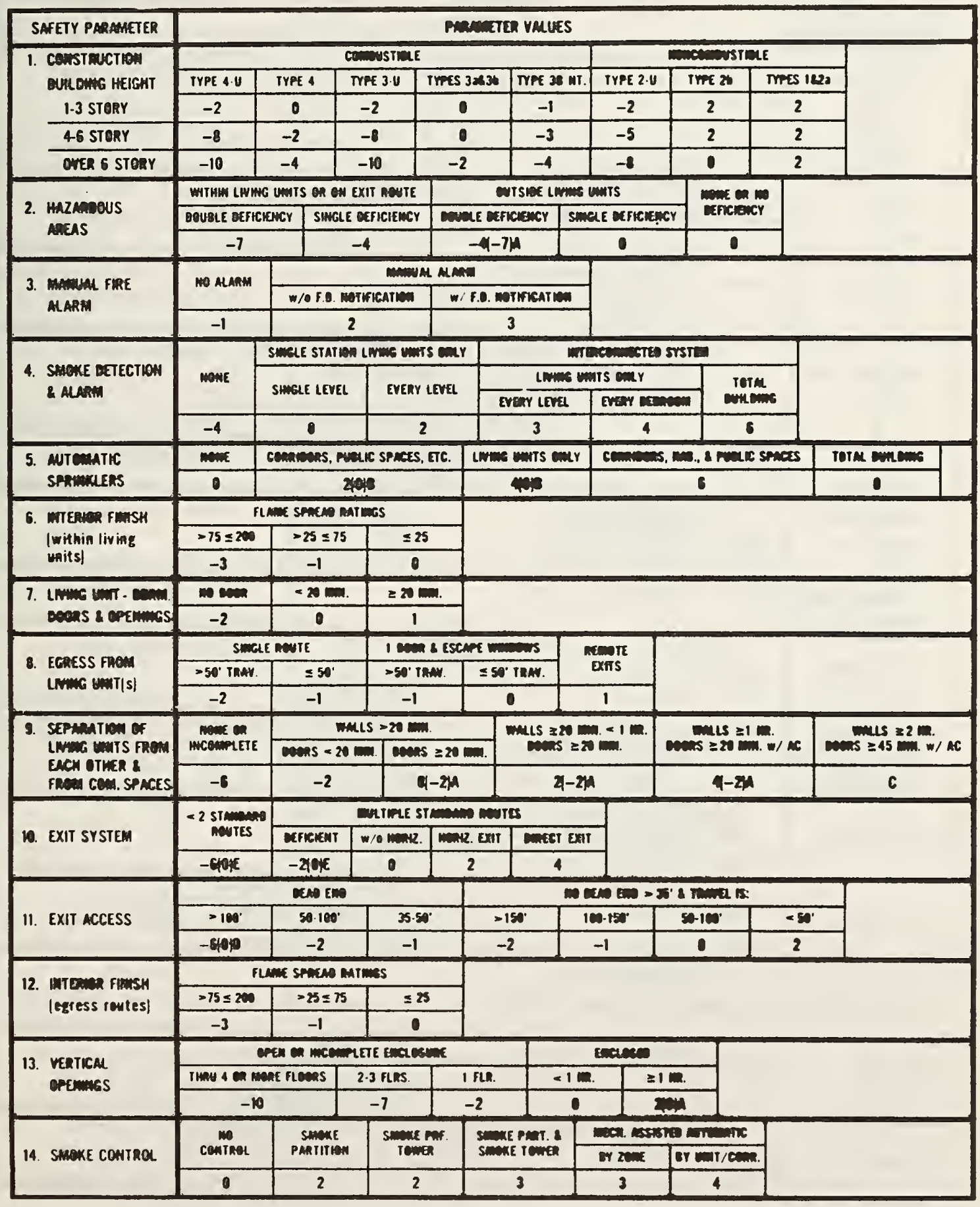

Figure 3. Safety Parameter Matrix 


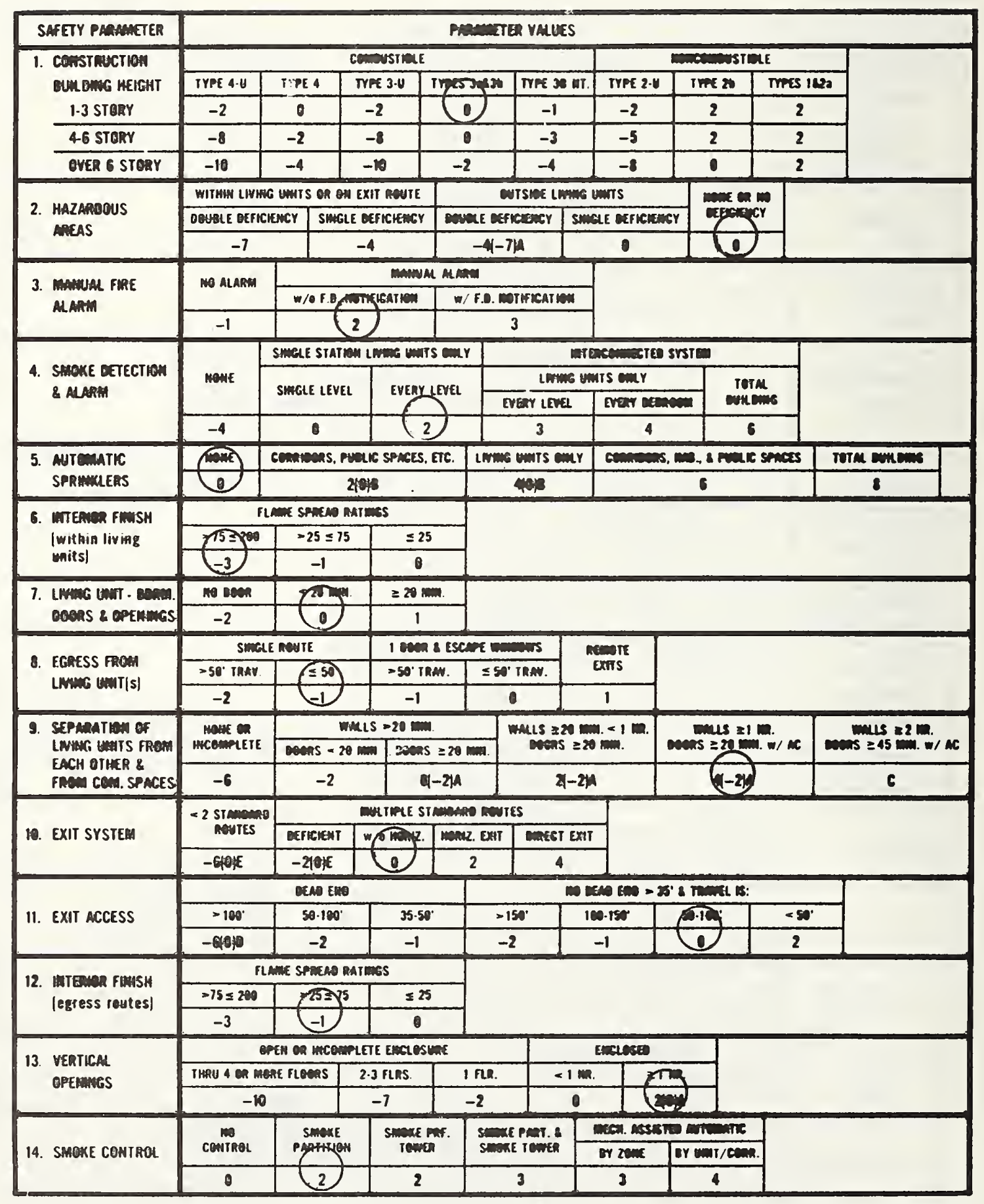

Figure 4a. MPS Requirements

Multifamily Housing

$\leq 3$ story $->8$ units/floor 


\begin{tabular}{|c|c|c|c|c|}
\hline SAFETY PAMAMETER & $\underset{\left|s_{1}\right|}{\operatorname{Fin}}$ & $\begin{array}{c}\text { Eestss } \\
\text { Promed } \\
{\left[S_{2} \mid\right.}\end{array}$ & $\begin{array}{l}\text { PEFueE } \\
\text { PROMUED } \\
\left|s_{3}\right|\end{array}$ & $\begin{array}{l}\text { GEMERAL FIPE } \\
\text { SAFETY PROWBED } \\
\text { (S4) }\end{array}$ \\
\hline 1. Construction & 0 & & 0 & 0 \\
\hline 2. HAZAMDOUS MAEAS & 0 & +20 & 0 & 0 \\
\hline 3. HAMUML FIDE ALAMA & $\div 21$ & 2 & & 2 \\
\hline $\begin{array}{l}\text { 4. SHOKE DETECTIEM \& } \\
\text { ALARA }\end{array}$ & $\div 2 \quad 1$ & 2 & & 2 \\
\hline 5. AUTOMATIC SPRMULERS & 0 & $\div 21000$ & $(\div 2) \mathrm{A} 0$ & 0 \\
\hline 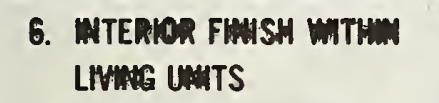 & $\div 2-1.5$ & & & -3 \\
\hline $\begin{array}{l}\text { 7. LIVHG UNIT - BDDA } \\
\text { DOORS \& OPEMUAS }\end{array}$ & & & 0 & 0 \\
\hline $\begin{array}{l}\text { 8. EGRESS FROA LIYHAG } \\
\text { UNTISI }\end{array}$ & & -1 & & -1 \\
\hline $\begin{array}{l}\text { 9. SEPARATION OF LIUMG } \\
\text { UAITS FROM EACH OTHER } \\
\text { \& FROA COMAMON SPACES }\end{array}$ & 4 & $\div 2 \quad 2$ & 4 & 4 \\
\hline 10. EXIT SYSTEMA & & 0 & $\div 20$ & 0 \\
\hline 11. EXIT ACCESS & & 0 & & 0 \\
\hline $\begin{array}{l}\text { 12. WTERIOR FINSH } \\
\text { (egress routes) }\end{array}$ & & -1 & & -1 \\
\hline 13. VERTICAL OPEMHISS & $\div 2 \quad 1$ & 2 & 2 & 2 \\
\hline 14. SMOKE CONTROL & & 2 & 2 & 2 \\
\hline TOTAL & $S_{1}=5.5$ & $S_{2}=8$ & $S_{3}=$ & $S_{4}=7$ \\
\hline \multicolumn{5}{|c|}{$\begin{array}{l}\text { MOTES: } \\
\text { A - Use full value if Safety Parameter } 1 \text { is based on } 2-U, 3-U \text { or } 4-U \text { construction. } \\
\text { Divide by } 2(\div 2) \text { in all other cases. } \\
\text { B - Use (0) if operation of sprinkler system does not activate building fire } \\
\text { alarm system. }\end{array}$} \\
\hline
\end{tabular}

Figure 4b. Mandatory Safety Requirements Multifamily Housing

$\leq 3$ story - > 8 units/floor 


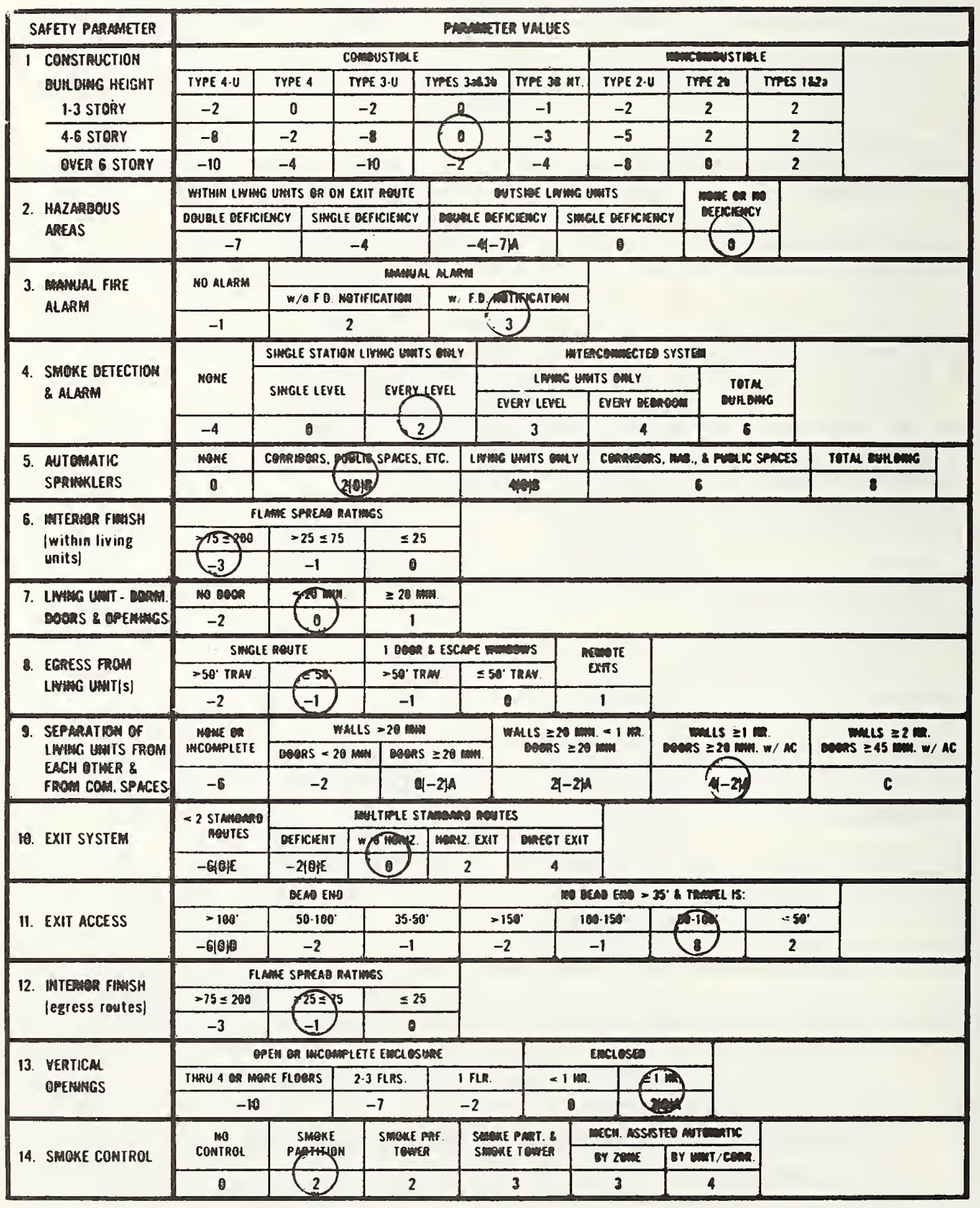

Figure 5a. MPS Requirements

Multifamily Housing

4-6 story $->8$ units/floor 


\begin{tabular}{|c|c|c|c|c|}
\hline SAFETY AMAMIETER & $\underset{\left[S_{1}\right]}{\text { Fism }}$ & 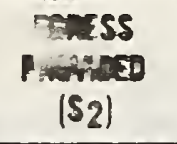 & $\begin{array}{c}\text { mexes } \\
\text { momacts } \\
\left(s_{3}\right)\end{array}$ & $\begin{array}{l}\text { GEMERA FIDE } \\
\text { SAFETY PIFYUDED } \\
\text { (S4) }\end{array}$ \\
\hline 1. Cowsthuction & 0 & & 0 & 0 \\
\hline 2. HZZMOOUS MEAS & 0 & +20 & 0 & 0 \\
\hline 3. MHULAL FIRE MLMM & $\div 2 \quad 1.5$ & 3 & & 3 \\
\hline 4. SUMXE BETECTIM \& & $\div 21$ & 2 & & 2 \\
\hline 5. ANTOMATIC SFRMULERS & 2 & $\div 2(0) 131$ & $(\div 2) \mathrm{A} 1$ & 2 \\
\hline $\begin{array}{l}\text { 6. MTERAOA FUHSH WTIM } \\
\text { LIVHG LAMTS }\end{array}$ & $\div 2-1.5$ & & & -3 \\
\hline $\begin{array}{l}\text { 7. LIVANG UAHT - BRAM } \\
\text { DOORS \& OPEMHAS }\end{array}$ & & & 0 & 0 \\
\hline $\begin{array}{l}\text { 8. EGRESS FROA LIVMM } \\
\text { UNIT/SI }\end{array}$ & & -1 & & -1 \\
\hline $\begin{array}{l}\text { 9. SEPARATION OF LIVHUG } \\
\text { UAITS FROM EACH OTHER } \\
\text { \& FROH COAMOA SPACES }\end{array}$ & 4 & $\div 2 \quad 2$ & 4 & 4 \\
\hline 10. EXIT SYSTEM & & 0 & $\div 2$ & 0 \\
\hline 11. EXT ACCESS & & 0 & & 0 \\
\hline $\begin{array}{l}\text { 12. HTERTOR FIAHSH } \\
\text { (egress routes) }\end{array}$ & & -1 & & -1 \\
\hline 13. VERTICAL OPENMUSS & $\div 21$ & 2 & 2 & 2 \\
\hline 14. SWOKE CONTROL & & 2 & 2 & 2 \\
\hline TOTAL & $S_{1}=8$ & $s_{2}=10$ & $S_{3}=9$ & $S_{4}=10$ \\
\hline $\begin{array}{l}\text { WOTES: } \\
\text { A - Use full value if Safet } \\
\text { Divide by } 2(\div 2) \text { in all } \\
\text { B - Use }(0) \text { if operation of } \\
\text { alarm system. }\end{array}$ & $\begin{array}{l}\text { Parameter } 1 \text { i } \\
\text { her cases. } \\
\text { riakler syste }\end{array}$ & $\begin{array}{l}\text { based on 2-U } \\
\text { does not ac }\end{array}$ & $\begin{array}{l}3-U \text { or } 4-U \mathrm{CC} \\
\text { vate building }\end{array}$ & istruction. \\
\hline
\end{tabular}

Figure 5b. Mandatory Safety Requirements

Multifamily Housing

4-6 story > 8 units/floor 


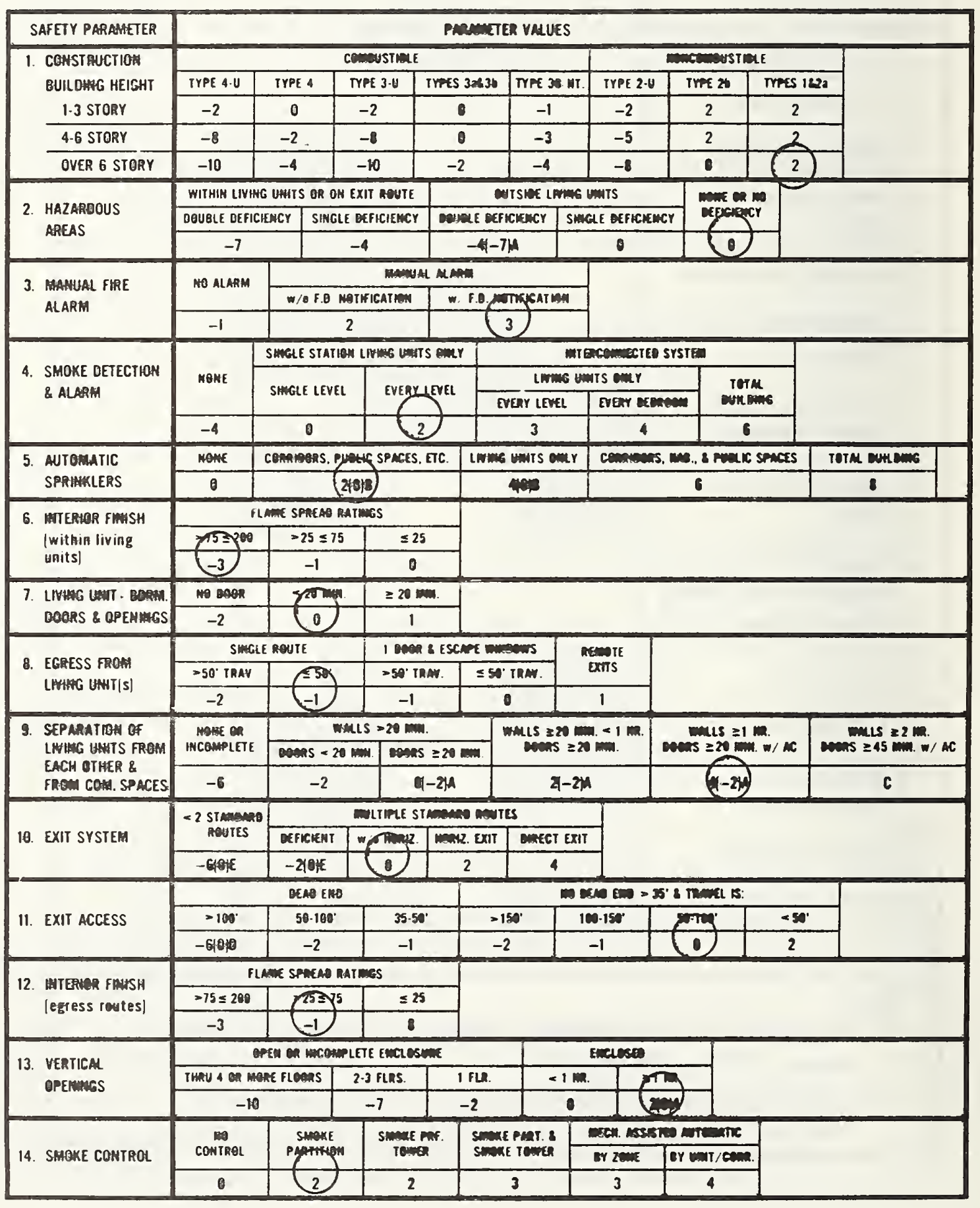

Figure 6a. MPS Requirements

Multifamily Housing

$>6$ story - > 8 units/floor 


\begin{tabular}{|c|c|c|c|c|}
\hline SAFETY PARAMETER & $\begin{array}{l}\text { Fine } \\
\text { cominae } \\
{\left[S_{1}\right]}\end{array}$ & $\begin{array}{l}\text { Ecruss } \\
\text { PRavezo } \\
{\left[S_{2}\right]}\end{array}$ & $\begin{array}{l}\text { PEFUCE } \\
\text { PRoNDED } \\
(\$ 3)\end{array}$ & $\begin{array}{l}\text { GEMERAL FIDE } \\
\text { SAFETY PROWDED } \\
\text { (S4) }\end{array}$ \\
\hline 1. CONSTRUCTION & $\dot{2}$ & & 2 & 2 \\
\hline 2. HAZMEOUS MNEAS & 0 & $\div 2 \quad 0$ & 0 & 0 \\
\hline 3. MAMUML FInE MAmm & $\div 2 \quad 1.5$ & 3 & & 3 \\
\hline $\begin{array}{l}\text { 4. SMOKE DETECTIMN \& } \\
\text { ALARIM }\end{array}$ & $\div 2 \quad 2$ & 2 & & 2 \\
\hline 5. AUTOAMATC SFAMULLERS & 1 & $\div 210981$ & $(\div 2) \mathrm{A} \quad 1$ & 2 \\
\hline $\begin{array}{l}\text { 6. HTERIOR FIASH WTHM } \\
\text { LIVHG UNTS }\end{array}$ & $\div 2 \quad 1.5$ & & & -3 \\
\hline $\begin{array}{l}\text { 7. LIVIAG UNT - BRRA } \\
\text { DOORS \& OPEIMISS }\end{array}$ & & & 0 & 0 \\
\hline $\begin{array}{l}\text { 8. EGRESS FROAM LIVHNG } \\
\text { UNIT(s) }\end{array}$ & & -1 & & -1 \\
\hline $\begin{array}{l}\text { 9. SEPARATHON OF LIVING } \\
\text { UNITS FROM EACH OTHER } \\
\text { \& FROA COMAON SPACES }\end{array}$ & 4 & $\div 2$ & 4 & 4 \\
\hline 10. EXIT SYSTEM & & 0 & $\div 2$ & 0 \\
\hline 11. EXIT ACCESS & & 0 & & 0 \\
\hline $\begin{array}{l}\text { 12. INTERAOR FIWSH } \\
\text { (egress routes] }\end{array}$ & & -1 & & -1 \\
\hline 13. VERTICAL OPENHWS & $\div 2$ & 2 & 2 & 2 \\
\hline 14. SMOKE CONTROL & & 2 & 2 & 2 \\
\hline TOTAL & $S_{1}=10$ & $S_{2}=10$ & $s_{3}=11$ & $S_{4}=$ \\
\hline \multicolumn{5}{|c|}{$\begin{array}{l}\text { NOTES: } \\
\text { A - Use full value if Safety Parameter } 1 \text { is based on } 2-U, 3-U \text { or } 4-U \text { construction. } \\
\text { Divide by } 2(\div 2) \text { in all other cases. } \\
\text { B - Use }(0) \text { if operation of sprinkler system does not activate building fire } \\
\text { alarm system. }\end{array}$} \\
\hline
\end{tabular}

Figure 6b. Mandatory Safety Requirements

Multifamily Housing

$>6$ story $>8$ units/floor 


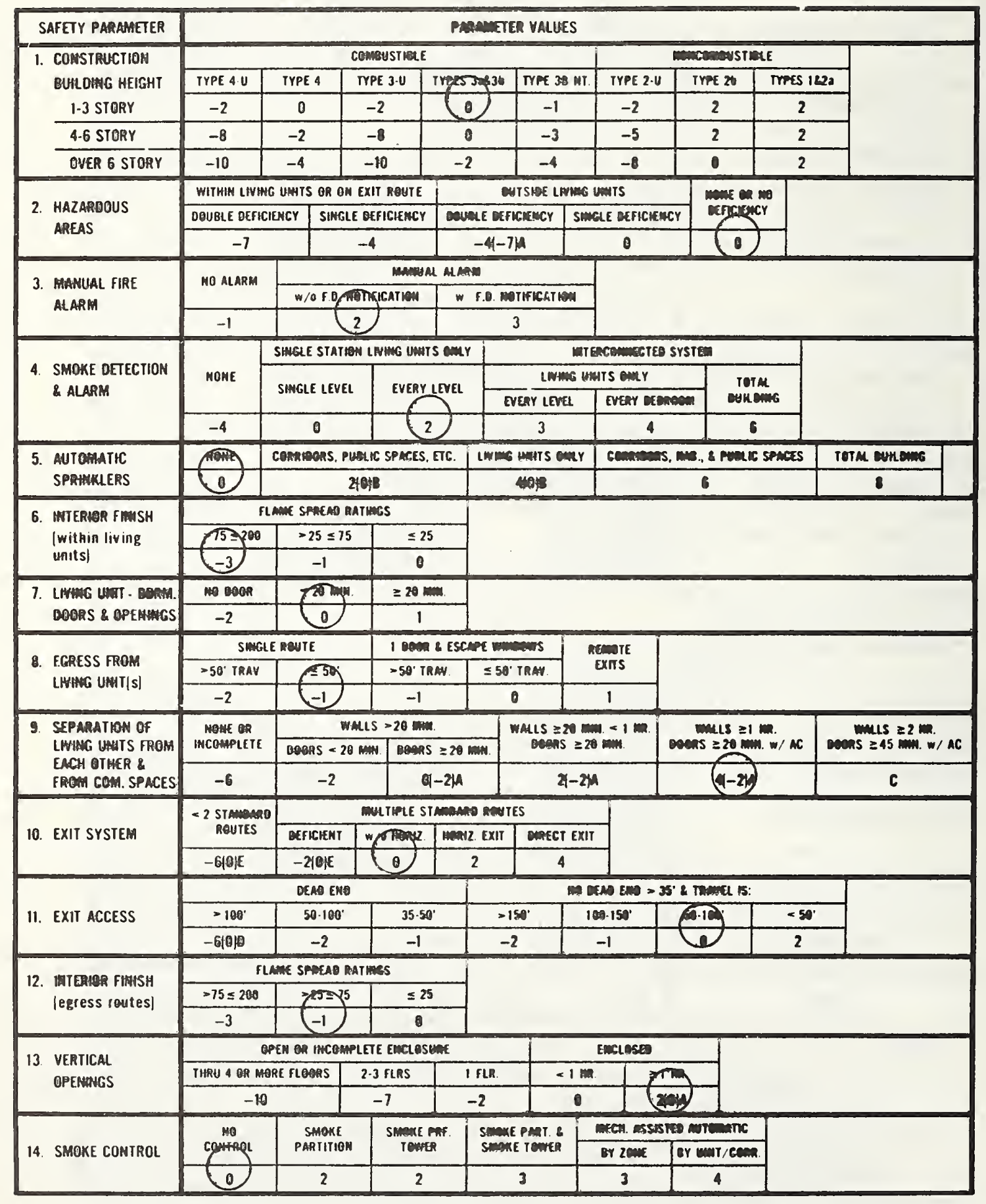

Figure 7a. MPS Requirements

Multifamily Housing

$\leq 3$ story $-\leq 8$ units/floor 


\begin{tabular}{|c|c|c|c|c|}
\hline SAFETY PAMAMETER & $\underset{\left[s_{1} \mid\right.}{\operatorname{comiz}}$ & 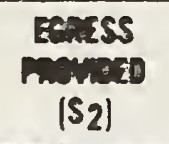 & 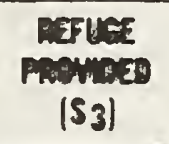 & $\begin{array}{l}\text { GELUEAML FIOE } \\
\text { SAFETY PAOMOED } \\
\text { (S4) }\end{array}$ \\
\hline 1. COASTRUCTIEH & 0 & & 0 & 0 \\
\hline 2. HAZnows ameAs & 0 & $\div 2$ & 0 & 0 \\
\hline 3. Мамиц FIE MUMm & $\div 21$ & 2 & & 2 \\
\hline $\begin{array}{l}\text { 4. SHEXE DETECTIEA \& } \\
\text { ALAMI }\end{array}$ & $\div 21$ & 2 & & 2 \\
\hline 5. AUTOMATIC SFmumLERS & 0 & $\div 21000$ & $1 \div 21 \mathrm{~A}_{0}$ & 0 \\
\hline 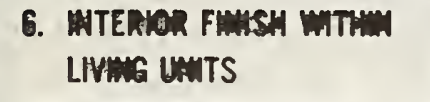 & $\div 2-1.5$ & & & -3 \\
\hline $\begin{array}{l}\text { 7. LIVHG UNT - BOAN } \\
\text { DOORS \& OPENMUSS }\end{array}$ & & & 0 & 0 \\
\hline $\begin{array}{l}\text { 8. EGPESS FROA LIVIMG } \\
\text { UWIT(s) }\end{array}$ & & -1 & & -1 \\
\hline $\begin{array}{l}\text { 9. SEPARATION OF LIUMG } \\
\text { UNITS FROA EACH OTHER } \\
\text { \& FROA COM }\end{array}$ & 4 & $\div 2$ & 4 & 4 \\
\hline 10. EXIT SYSTEH & & 0 & $\div 2$ & 0 \\
\hline 11. EXTT ACCESS & & 0 & & 0 \\
\hline $\begin{array}{l}\text { 12. ATERTOR FIAMSH } \\
\text { (egress routes) }\end{array}$ & & -1 & & -1 \\
\hline 13. VERTCAL OPEAMUSS & $\div 2 \quad 1$ & 2 & 2 & 2 \\
\hline 14. SMOKE CONTROL & (1: & 0 & 0 & 0 \\
\hline TOTAL & $s_{1}=5.5$ & $s_{2}=$ & $S_{3}=6$ & $S_{4}=$ \\
\hline \multicolumn{5}{|c|}{$\begin{array}{l}\text { MOTES: } \\
\text { A - Use full value if Safety Parameter } 1 \text { is based on 2-U, 3-U or } 4-U \text { construction. } \\
\text { Divide by } 2[\div 2) \text { in all other cases. } \\
\text { B - Use (0) if operation of sprinkler system does not activate building fire } \\
\text { alarm system. }\end{array}$} \\
\hline
\end{tabular}

Figure 7b. Mandatory Safety Requirements Multifamily Housing

$\leq 3$ story $\leq 8$ units/floor 


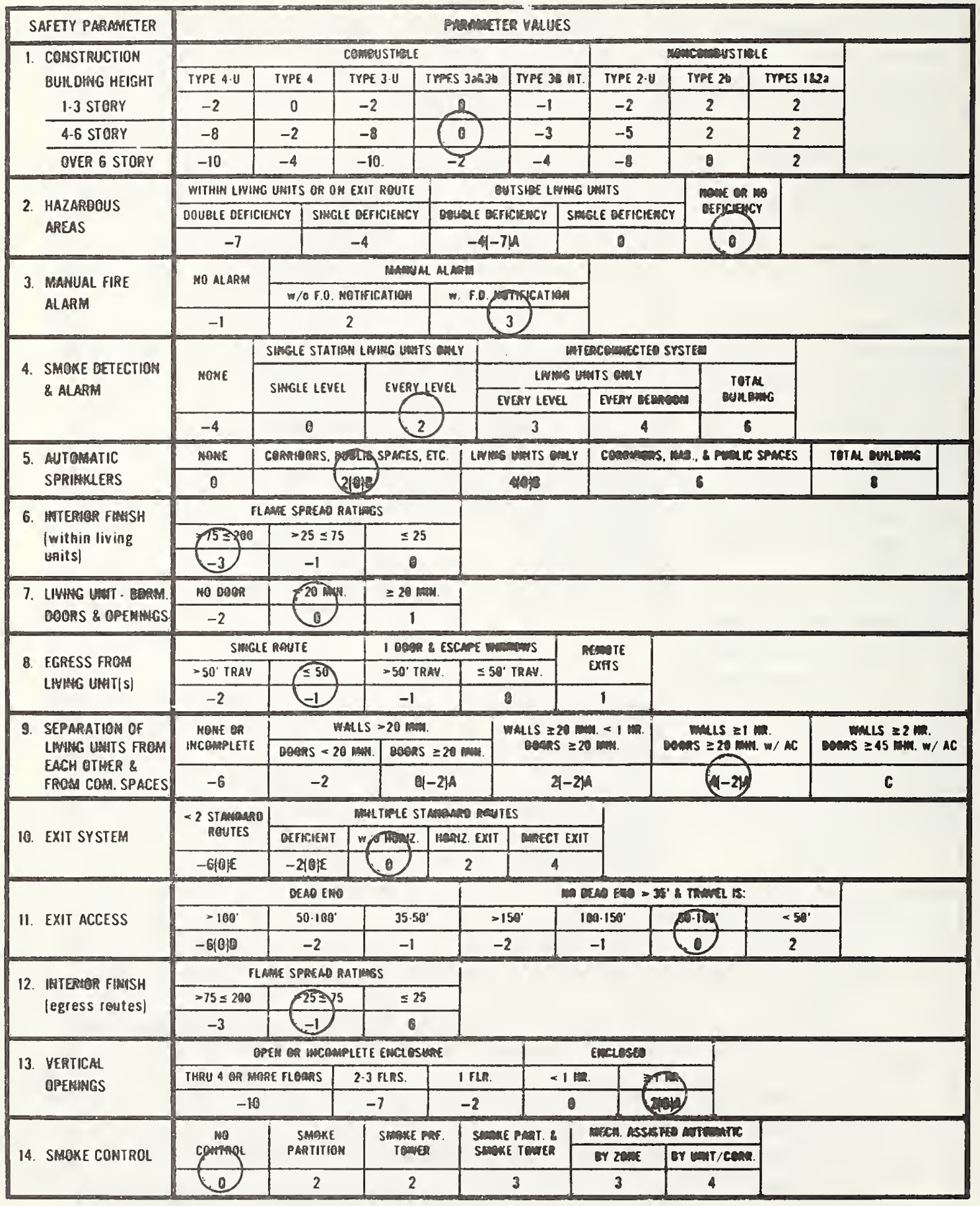

Figure 8a. MPS Requirements

Multifamily Housing

4-6 story $-\leq 8$ unit/floor 


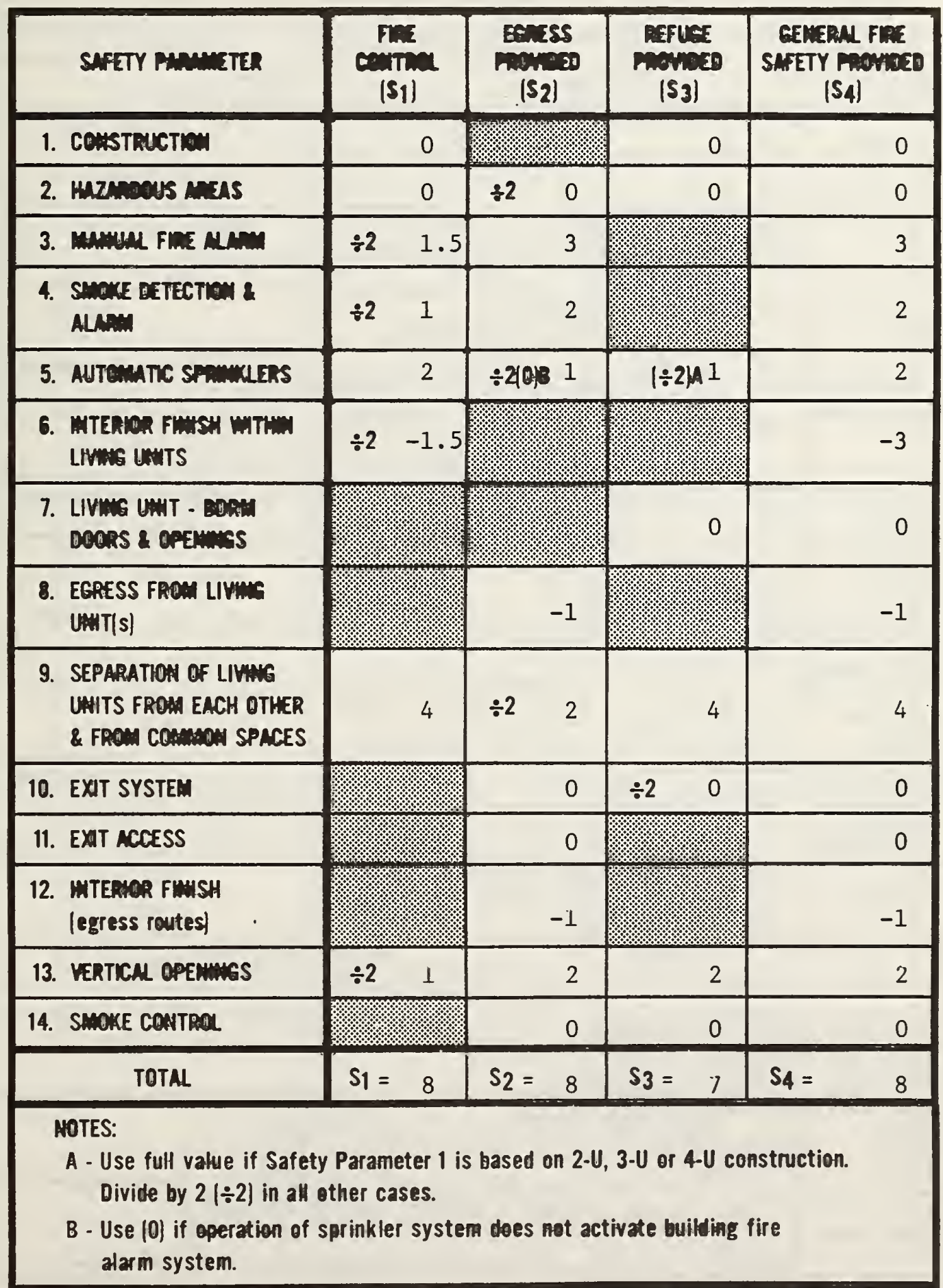

Figure 8b. Mandatory Safety Requirements Multifamily Housing 4-6 story - $\leq 8$ units/floor 


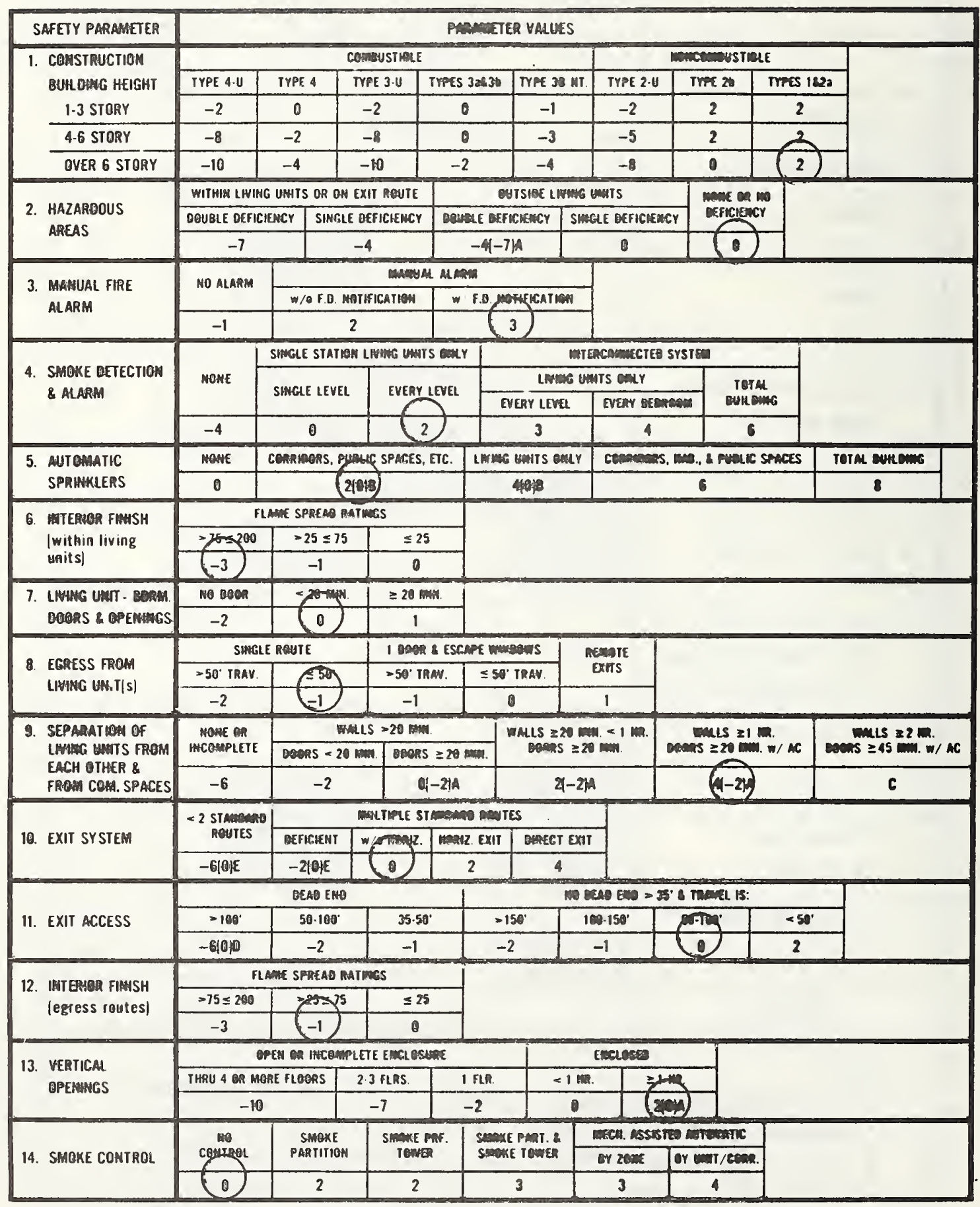

Figure 9a. MPS Requirements

Multifamily Housing

$>6$ story $-\leq 8$ units/floor 


\begin{tabular}{|c|c|c|c|c|}
\hline SAFETY PAMAMETER & $\underset{\left|s_{1}\right|}{\operatorname{conim}}$ & $\begin{array}{l}\text { EenEss } \\
\text { Pmimat } \\
{\left[S_{2} \mid\right.}\end{array}$ & 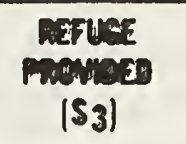 & $\begin{array}{l}\text { GEHEAM FITE } \\
\text { SAFETY PROWAED } \\
\text { (SA) }\end{array}$ \\
\hline 1. COHSTRUCTIOH & 2 & & 2 & 2 \\
\hline 2. HAZMmous MMEAS & 0 & $\div 2$ & 0 & 0 \\
\hline 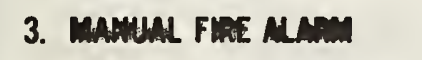 & $\div 2 \quad 1.5$ & 3 & 㤅 & 3 \\
\hline $\begin{array}{l}\text { 4. SMEXE DETECTEA \& } \\
\text { ALAMI }\end{array}$ & $\div 2 \quad 1$ & 2 & & 2 \\
\hline 5. AUTCMATIC SPMUMALERS & 2 & $\div 21018 \quad 1$ & $(\div 2) A_{1}$ & 2 \\
\hline 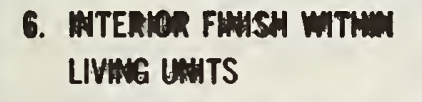 & $\div 2-1.5$ & & & -3 \\
\hline $\begin{array}{l}\text { 7. LIVHAG UNT - RORH } \\
\text { DOORS \& OPEMWGS }\end{array}$ & & & 0 & 0 \\
\hline $\begin{array}{l}\text { 8. EGRESS FROM LIVIMAC } \\
\text { UANT(S) }\end{array}$ & & -1 & & -1 \\
\hline $\begin{array}{l}\text { 9. SEPARATION OF LIVAMG } \\
\text { UAITS FROM EACH OTHER } \\
\text { \& FR COM SPACES }\end{array}$ & 4 & $\div 2$ & 4 & 4 \\
\hline 10. EXIT SYSTEM & 网 & 0 & $\div 2$ & 0 \\
\hline 11. EXIT ACCESS & 痹 & 0 & & 0 \\
\hline $\begin{array}{l}\text { 12. NTEAHOR FIA-SH } \\
\text { (egress routes| }\end{array}$ & & -1 & & -1 \\
\hline 13. VERTICAL OPEAHMS & $\div 2 \quad 1$ & 2 & 2 & 2 \\
\hline 14. SHOKE CONTROL & & 0 & 0 & 0 \\
\hline TOTAL & $S_{1}=10$ & $s_{2}=$ & $s_{3}=$ & $S_{4}=$ \\
\hline \multicolumn{5}{|l|}{$\begin{array}{l}\text { MOTES: } \\
\text { A - Use full vatue if Safe } \\
\text { Divide by } 2(\div 2) \text { in all }\end{array}$} \\
\hline
\end{tabular}

Figure 9b. Mandatory Safety Requirements Multifamily Housing $>6$ story $-\leq 8$ units/floor 


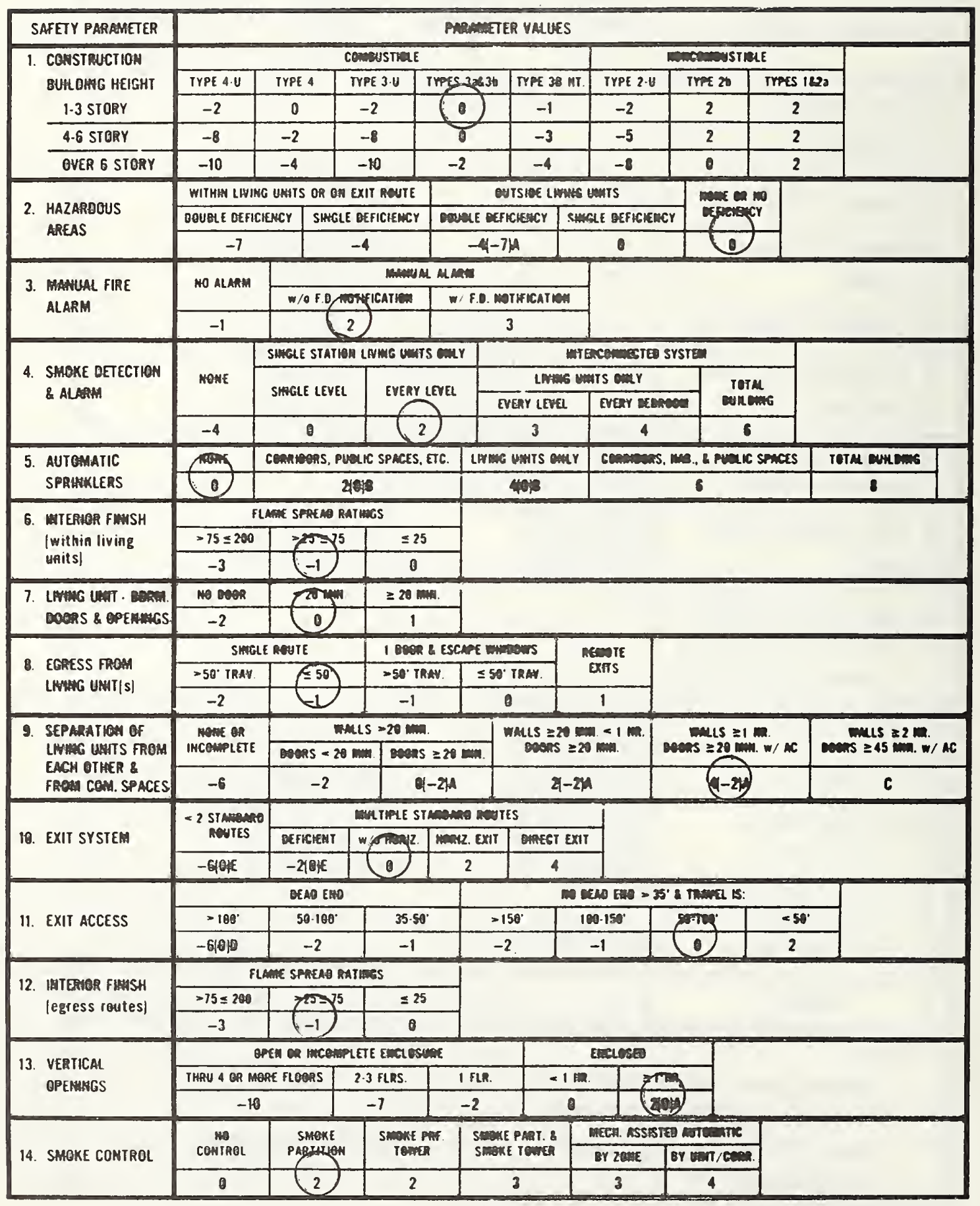

Figure 10a. MPS Requirements

Housing for the Elderly

$\leq 3$ story - > 8 units/floor 


\begin{tabular}{|c|c|c|c|c|}
\hline SAFETY PAMMETER & $\underset{\left(S_{1}\right)}{\operatorname{Fin}}$ & 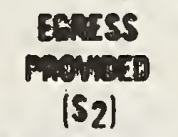 & 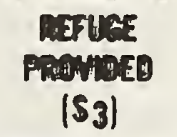 & $\begin{array}{l}\text { GEEERRM FIDE } \\
\text { SAFETY PROWBEO } \\
\text { (S4) }\end{array}$ \\
\hline 1. constauction & $\dot{0}$ & & 0 & 0 \\
\hline 2. HIZnarus MEAS & 0 & $\div 2$ & 0 & 0 \\
\hline 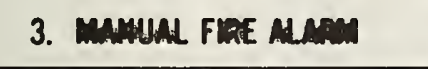 & $\div 2 \quad 1$ & 2 & & 2 \\
\hline $\begin{array}{l}\text { 4. SUaxe BETECTIAH \& } \\
\text { AUMH }\end{array}$ & $\div 2 \quad 1$ & 2 & & 2 \\
\hline 5. AUTOMATKC SPTLAKLERS & 0 & $\div 210 \mid 180$ & $(\div 2) A 0$ & 0 \\
\hline $\begin{array}{l}\text { 6. HTERIER FHASH WTHM } \\
\text { LIWHG UATS }\end{array}$ & $\div 2 \quad-.5$ & & & -1 \\
\hline $\begin{array}{l}\text { 7. LIVING UNT - BOAM } \\
\text { DOORS \& OPEMWMS }\end{array}$ & & & 0 & 0 \\
\hline $\begin{array}{l}\text { 8. EGRESS FROM LIVMUG } \\
\text { UAMT/S) }\end{array}$ & & -1 & & -1 \\
\hline $\begin{array}{l}\text { 9. SEPARATION OF LIVIAG } \\
\text { UAITS FROM EACH OTHER } \\
\text { \& FROH COWMOA SPACES }\end{array}$ & 4 & $\div 2$ & 4 & 4 \\
\hline 10. EXIT SYSTEM & & 0 & $\div 2$ & 0 \\
\hline 11. EXT ACCESS & & 0 & & 0 \\
\hline $\begin{array}{l}\text { 12. IMTERTOR FIASH } \\
\text { (egress routes) }\end{array}$ & & -1 & & -1 \\
\hline 13. VERTICAL OPEAMHGS & $\div 2 \quad 1$ & 2 & 2 & 2 \\
\hline 14. SMOKE CONTROL & & 2 & 2 & 2 \\
\hline TOTAL & $S_{1}=6.5$ & $S_{2}=8$ & $s_{3}=8$ & $S_{4}=$ \\
\hline $\begin{array}{l}\text { NOTES: } \\
\text { A - Use full value if Safe } \\
\text { Divide by } 2(\div 2) \text { in at } \\
\text { B - Use }(0) \text { if operation of } \\
\text { alarm system. }\end{array}$ & $\begin{array}{l}\text { Parameter } 1 \text { is } \\
\text { her cases. } \\
\text { rinkler syster }\end{array}$ & $\begin{array}{l}\text { based on } 2-1 \\
\text { dees not ac }\end{array}$ & $\begin{array}{l}3-U \text { or } 4-U \mathrm{C} \\
\text { vate building }\end{array}$ & $\begin{array}{l}\text { nstruction. } \\
\text { fire }\end{array}$ \\
\hline
\end{tabular}

Figure 10b. Mandatory Safety Requirements Housing for the Elderly $\leq 3$ story - > 8 units/floor 


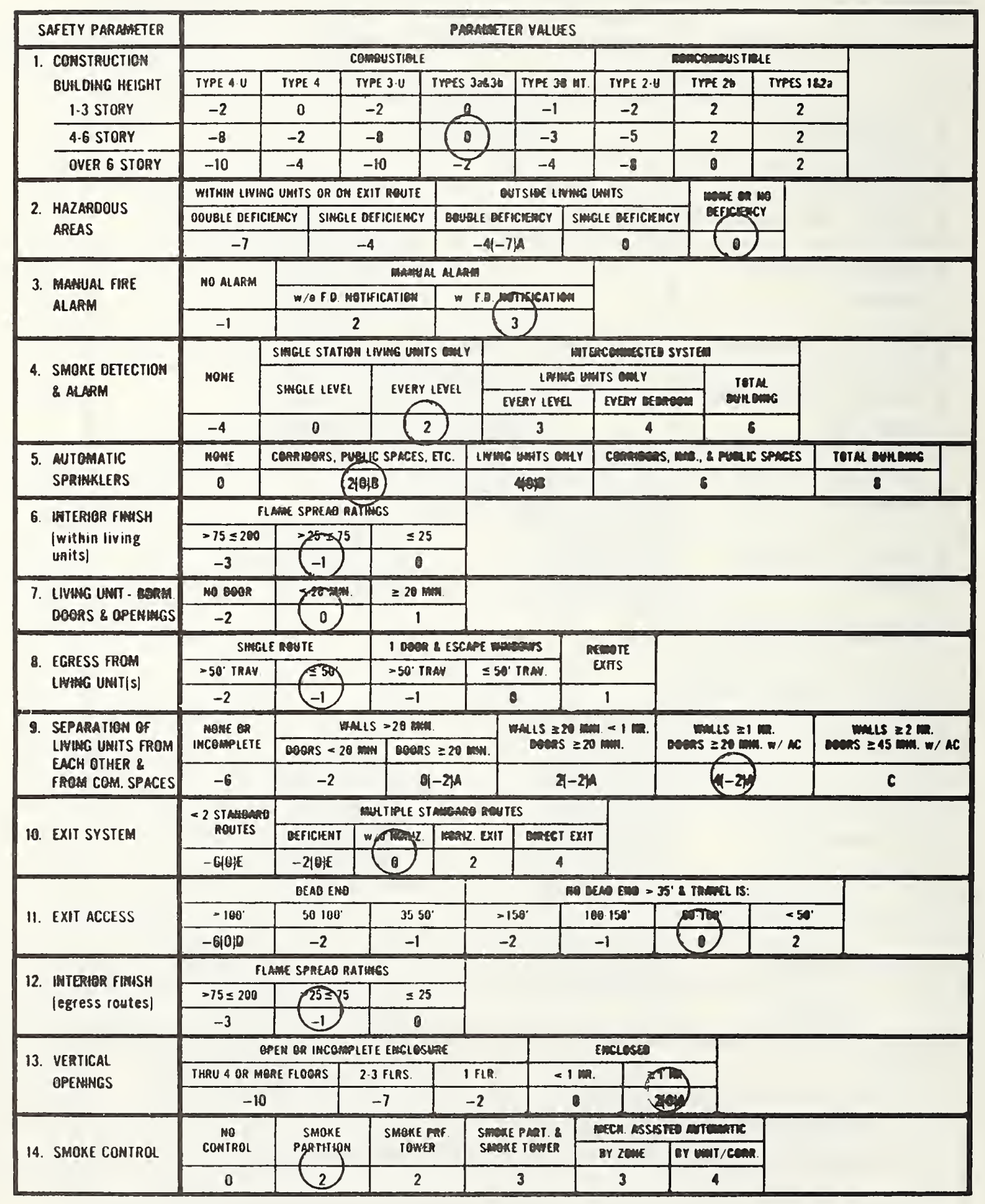

Figure 11a. MPS Requirements

Housing for the Elderly

4-6 story - > 8 units/floor 


\begin{tabular}{|c|c|c|c|c|}
\hline SAFETY PANAMETER & 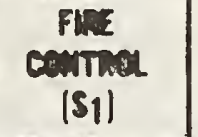 & 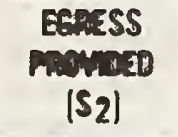 & 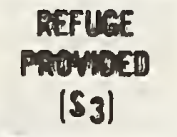 & $\begin{array}{l}\text { GENERAL FIPE } \\
\text { SAFETY PRBMDED } \\
\text { (S4) }\end{array}$ \\
\hline 1. COAstruction & 0 & & 0 & 0 \\
\hline 2. MAZAmBaUS APHS & 0 & $\div 2$ & 0 & 0 \\
\hline 3. МANULL FIRE MANA & $\div 2 \quad 1.5$ & 3 & & 3 \\
\hline $\begin{array}{l}\text { 4. SUQRE BETECTIEN \& } \\
\text { ALAMA }\end{array}$ & $\div 2$ & 2 & & 2 \\
\hline 5. AUTOMATIC SPRMULLRS & 2 & $\div 2|0| B \quad 1$ & $(\div 2) A 1$ & 2 \\
\hline $\begin{array}{l}\text { 6. WTERIOR FIAISH MTTMM } \\
\text { LIUANG LAITS }\end{array}$ & $\div 2 \quad-.5$ & & & -1 \\
\hline $\begin{array}{l}\text { 7. LIVHG UAT - BORM } \\
\text { DOORS \& OPEMMISS }\end{array}$ & & & 0 & 0 \\
\hline $\begin{array}{l}\text { 8. EGRESS FROM LIVMAG } \\
\text { UNIT/S] }\end{array}$ & & -1 & & -1 \\
\hline $\begin{array}{l}\text { 9. SEPARATION OF LIVMAG } \\
\text { UAITS FROM EACH OTHER } \\
\text { \& FROM COMMOM SPACES }\end{array}$ & 4 & $\div 2$ & 4 & 4 \\
\hline 10. EXIT SYSTEM & & 0 & $\div 2$ & 0 \\
\hline 11. EXIT ACCESS & & 0 & & 0 \\
\hline $\begin{array}{l}\text { 12. INTERIOR FINSH } \\
\text { legress routes| }\end{array}$ & & -1 & & -1 \\
\hline 13. VERTICAL OPEAHAGS & $\div 2$ & 2 & 2 & 2 \\
\hline 14. SHOKE CONTROL & & 2 & 2 & 2 \\
\hline TOTAL & $S_{1}=9$ & $s_{2}=10$ & $S_{3}=9$ & $S_{4}=$ \\
\hline \multicolumn{5}{|c|}{$\begin{array}{l}\text { NOTES: } \\
\text { A - Use full value if Safety Parameter } 1 \text { is based on } 2-U, 3-U \text { or } 4-U \text { construction. } \\
\text { Divide by } 2(\div 2) \text { in all other cases. } \\
\text { B - Use (0) if operation of sprinkler system does not activate building fire } \\
\text { alarm system. }\end{array}$} \\
\hline
\end{tabular}

Figure 11b. Mandatory Safety Requirements Housing for the Elderly 4-6 story > 8 units/floor 


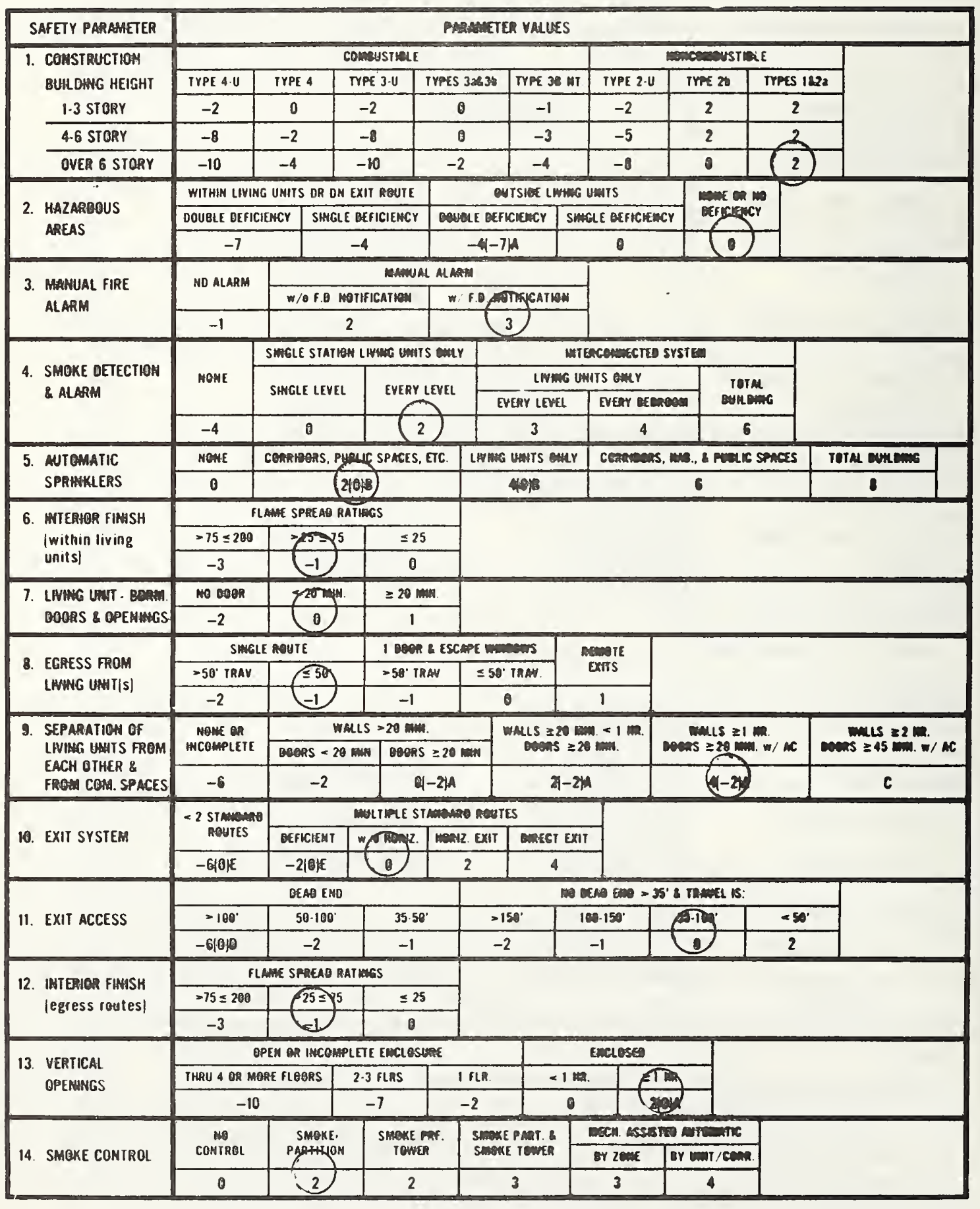

Figure 12a. MPS Requirements

Housing for the Elderly

$>6$ story - > 8 units/floor 


\begin{tabular}{|c|c|c|c|c|}
\hline SAFETY PANAMTER & $\begin{array}{l}\text { Fing } \\
\text { coitinat } \\
\left|s_{1}\right|\end{array}$ & $\begin{array}{l}\text { Eencess } \\
\text { finvisd } \\
{\left[\$_{2}\right]}\end{array}$ & $\begin{array}{l}\text { PEFUEE } \\
\text { PROHISB } \\
\left(\$_{3}\right)\end{array}$ & $\begin{array}{l}\text { GEAERAL FHEE } \\
\text { SAFETY PROVOED } \\
\text { (S4) }\end{array}$ \\
\hline 1. ConstRuction & 2 & & 2 & 2 \\
\hline 2. HAZARDOUS AREAS & 0 & $\div 2$ & 0 & 0 \\
\hline 3. MUHUA FLE MMP & $\div 2 \quad 1.5$ & 3 & & 3 \\
\hline $\begin{array}{l}\text { 4. SHGKE BETECTION \& } \\
\text { ALAEA }\end{array}$ & $\div 2$ & 2 & & 2 \\
\hline 5. AUTOMATC SPMULERS & 2 & $\div 2(0) B 1$ & $(\div 2) A_{1}$ & 2 \\
\hline 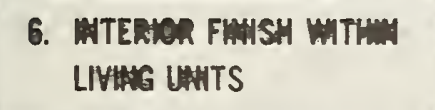 & $\div 2 \quad-.5$ & & & -1 \\
\hline $\begin{array}{l}\text { 7. LIVHG LAHT - BDPM } \\
\text { DOORS \& OPEAMASS }\end{array}$ & & & 0 & 0 \\
\hline $\begin{array}{l}\text { 8. EGRESS FROM LIVMM } \\
\text { UAIT|S| }\end{array}$ & & -1 & & -1 \\
\hline $\begin{array}{l}\text { 9. SEPARATON OF LIUAAG } \\
\text { UAITS FRON EACH OTHER } \\
\text { \& FROA COMHON SPACES }\end{array}$ & 4 & $\div 2$ & 4 & 4 \\
\hline 10. EXIT SYSTEMA & & 0 & $\div 2$ & 0 \\
\hline 11. EXIT ACCESS & & 0 & & 0 \\
\hline $\begin{array}{l}\text { 12. WTEROR FHASH } \\
\text { (egresS routes) }\end{array}$ & & -1 & & -1 \\
\hline 13. VERTICAL OPEAMUS & $\div 2$ & 2 & 2 & 2 \\
\hline 14. SAOKE CONTROL & & 2 & 2 & 2 \\
\hline TOTAL & $S_{1}=11$ & $s_{2}=10$ & $s_{3}=11$ & $S_{4}=$ \\
\hline $\begin{array}{l}\text { NOTES: } \\
\text { A - Use full value if Safet } \\
\text { Divide by } 2[\div 2] \text { in all } \\
\text { B - Use }(0) \text { if operation of } \\
\text { alarm system. }\end{array}$ & $\begin{array}{l}\text { arameter } 1 \text { is } \\
\text { ner cases. } \\
\text { rinkler systen }\end{array}$ & $\begin{array}{l}\text { based on } 2-U \\
\text { does not ac }\end{array}$ & 3.U or $4-U \mathrm{C}$ & $\begin{array}{l}\text { Istruction. } \\
\text { ire }\end{array}$ \\
\hline
\end{tabular}

Figure 12b. Mandatory Safety Requirements

Housing for the Elderly

$>6$ story - > 8 units/floor 


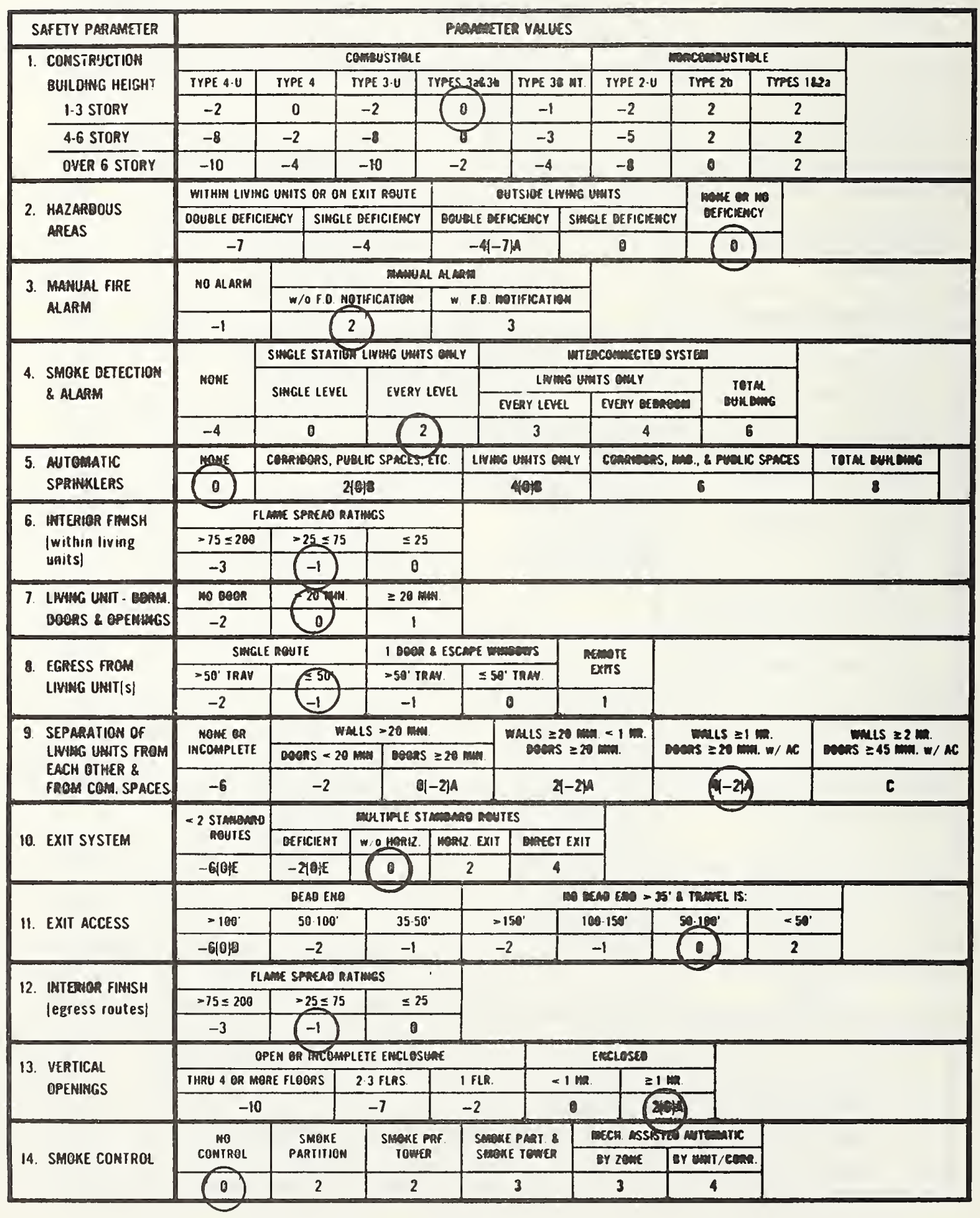

Figure 13a. MPS Requirements

$$
\begin{aligned}
& \text { Housing for the Elderly } \\
& \leq 3 \text { story }-\leq 8 \text { units/floor }
\end{aligned}
$$




\begin{tabular}{|c|c|c|c|c|c|c|}
\hline SAFETY PARAMETER & Com & & Eans & & $\begin{array}{l}\text { REFHE } \\
\text { PRONDED } \\
\text { (\$3) }\end{array}$ & $\begin{array}{l}\text { GEREPAML FIOE } \\
\text { SAFETY PROHOED } \\
\text { (S4) }\end{array}$ \\
\hline 1. comsteructien & & 0 & & & 0 & 0 \\
\hline 2. HAZAMEaUS AMEAS & & 0 & $\div 2$ & 0 & 0 & 0 \\
\hline 3. MANLAL FIEE MAAT & $\div 2$ & 1 & & 2 & & 2 \\
\hline $\begin{array}{l}\text { 4. SHAKE DETECTIGW \& } \\
\text { ALARM }\end{array}$ & $\div 2$ & 1 & & 2 & & 2 \\
\hline 5. AUTOMATIC SPFAMULLERS & & 0 & $\div 2 / 018$ & 0 & $1 \div 2 \mid \mathrm{A} 0$ & 0 \\
\hline $\begin{array}{l}\text { 6. WTERIOA FIMISH WTHN } \\
\text { LIVHA UNHTS }\end{array}$ & $\div 2$ & -.5 & & & & -1 \\
\hline $\begin{array}{l}\text { 7. LIVIAG UNIT - BORHA } \\
\text { DOORS \& OPEMMGS }\end{array}$ & & & & & 0 & 0 \\
\hline $\begin{array}{l}\text { 8. EGRESS FROA LIVIACE } \\
\text { UNIT(S) }\end{array}$ & & & & -1 & & -1 \\
\hline $\begin{array}{l}\text { 9. SEPARATION OF LIVING } \\
\text { UNITS FROM EACH OTHER } \\
\text { \& FROM COAON SPACES }\end{array}$ & & 4 & $\div 2$ & 2 & 4 & 4 \\
\hline 10. EXIT SYSTEM & & & & 0 & $\div 2$ & 0 \\
\hline 11. EXIT ACCESS & & & & 0 & & 0 \\
\hline $\begin{array}{l}\text { 12. INTER+OR FINISH } \\
\text { (egress routes) }\end{array}$ & & & & -1 & & -1 \\
\hline 13. VERTICAL OPEHAGS & & 1 & & 2 & 2 & 2 \\
\hline 14. SHOKE CONTROL & & & & 0 & 0 & 0 \\
\hline TOTAL & $s_{1}=$ & 6.5 & $S_{2}=$ & 6 & $S_{3}=6$ & $S_{4}=$ \\
\hline \multicolumn{7}{|c|}{$\begin{array}{l}\text { NOTES: } \\
\text { A - Use full value if Safety Parameter } 1 \text { is based on } 2-U, 3-U \text { or } 4-U \text { construction. } \\
\text { Divide by } 2(\div 2) \text { in all other cases. }\end{array}$} \\
\hline
\end{tabular}

Figure 13b. Mandatory Safety Requirements

Housing for the Elderly

$\leq 3$ story $-\leq 8$ units/floor 


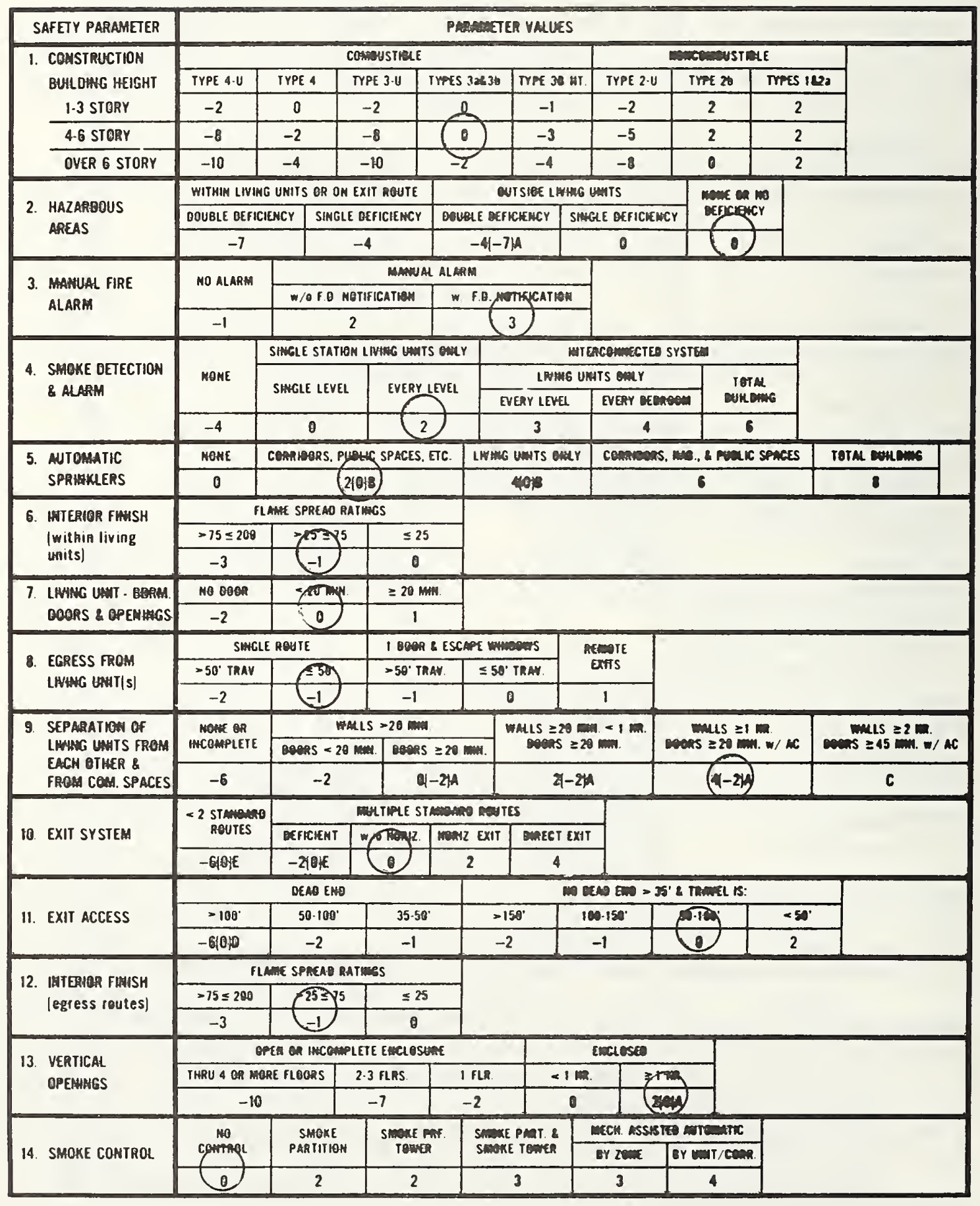

Figure 14a. MPS Requirements

Housing for the Elderly

4-6 story $-\leq 8$ units/floor 


\begin{tabular}{|c|c|c|c|c|c|c|}
\hline SHFETY PAMAMETER & centin & & $\begin{array}{l}\text { Eegess } \\
\text { Prowneg } \\
{\left[S_{2} \mid\right.}\end{array}$ & & $\begin{array}{l}\text { REFUEE } \\
\text { PMONDED } \\
\text { (\$ } 3 \text { ) }\end{array}$ & $\begin{array}{l}\text { GEAERAL FIDE } \\
\text { SAFETY PROMBED } \\
\text { (S4) }\end{array}$ \\
\hline 1. CONSTRUCTIOA & & 0 & 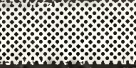 & & 0 & 0 \\
\hline 2. HMZARBQUS MEEAS & & 0 & $\div 2$ & 0 & 0 & 0 \\
\hline 3. MAMUUL FIRE MLAM & & 1.5 & & 3 & & 3 \\
\hline $\begin{array}{l}\text { 4. SHOKE DETECTION \& } \\
\text { ALARE }\end{array}$ & $\div 2$ & 1 & & 2 & & 2 \\
\hline 5. AUTOMATIC SPRWMLLERS & & 2 & $\div 21018$ & 1 & {$[\div 2\} \mathrm{A} \quad 1$} & 2 \\
\hline 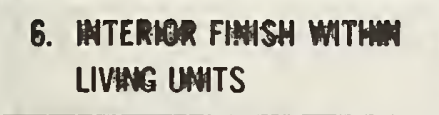 & & -.5 & & & & -1 \\
\hline $\begin{array}{l}\text { 7. LIVING UNIT - BPPA } \\
\text { DOORS \& OPEAMASS }\end{array}$ & & & & & 0 & 0 \\
\hline $\begin{array}{l}\text { 8. EGRESS FROMA LIVIHG } \\
\text { UNIT(S) }\end{array}$ & & & & -1 & & -1 \\
\hline $\begin{array}{l}\text { 9. SEPARATION OF LIVING } \\
\text { UNITS FROM EACH OTHER } \\
\text { \& FROM COMMON SPACES }\end{array}$ & & 4 & & 2 & 4 & 4 \\
\hline 10. EXIT SYSTEM & & & & 0 & $\div 2$ & 0 \\
\hline 11. EXIT ACCESS & & & & 0 & & 0 \\
\hline $\begin{array}{l}\text { 12. INTERIOR FIWHSH } \\
\text { (egress routes) }\end{array}$ & & & & -1 & & -1 \\
\hline 13. VERTICAL OPEAMAGS & $\div 2$ & $\perp$ & & 2 & 2 & $\dot{2}$ \\
\hline 14. SMOKE CONTROL. & & 原 & & 0 & 0 & 0 \\
\hline TOTAL & $S_{1}=$ & 9 & $s_{2}=$ & 8 & $s_{3}=$ & $S_{4}=$ \\
\hline \multicolumn{7}{|c|}{$\begin{array}{l}\text { MOTES: } \\
\text { A - Use full value if Safety Parameter } 1 \text { is based on } 2-U, 3-U \text { or } 4-U \text { construction. } \\
\text { Divide by } 2(\div 2) \text { in all other cases. } \\
\text { B - Use }(0) \text { if eperation of sprinkler system does net activate building fire } \\
\text { alarm system. }\end{array}$} \\
\hline
\end{tabular}

Figure 14b. Mandatory Safety Requirements Housing for the Elderly 4-6 story $-\leq 8$ units/floor 


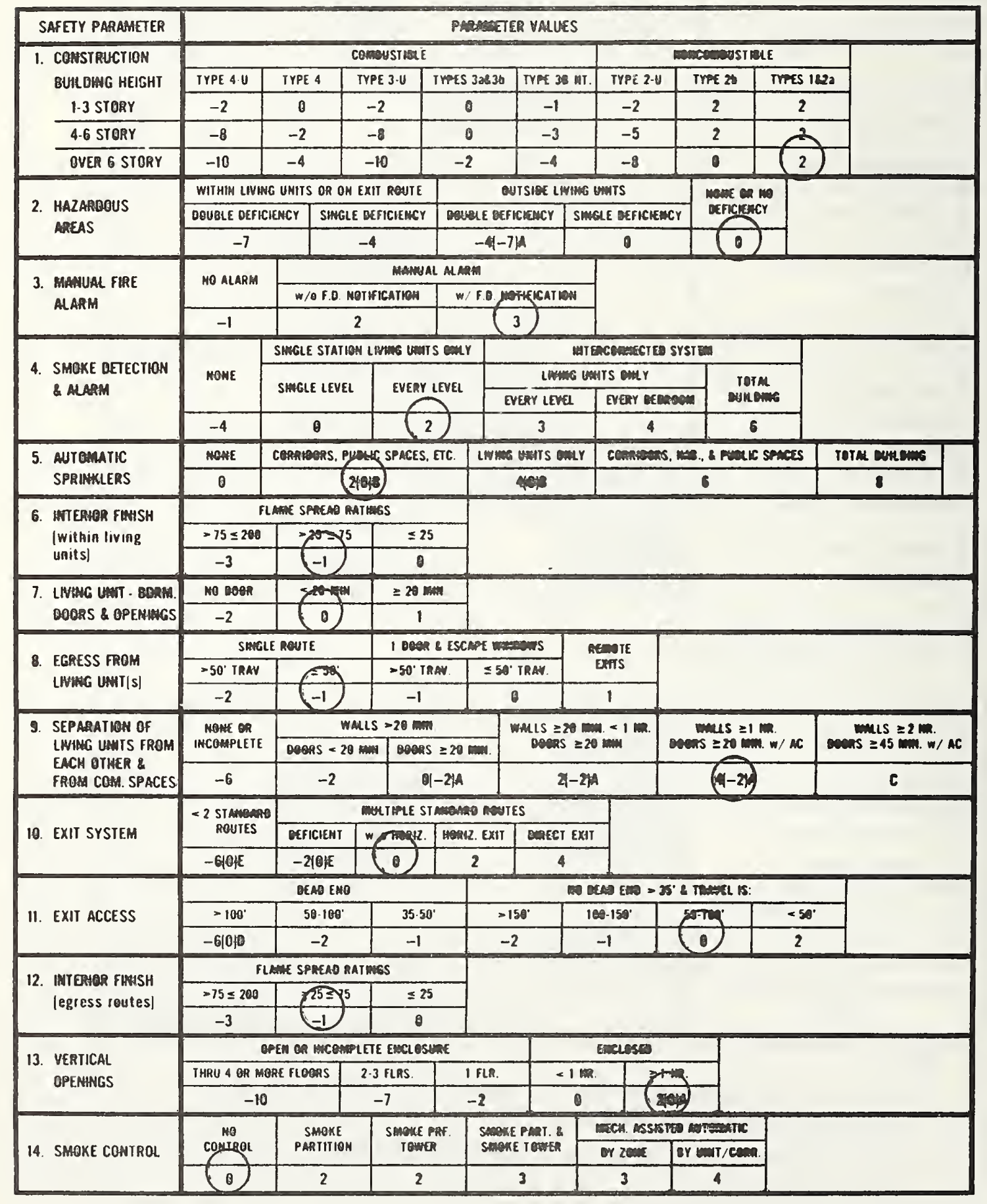

Figure 15a. MPS Requirements

Housing for the Elderly

$>6$ story $-\leq 8$ units/floor 


\begin{tabular}{|c|c|c|c|c|}
\hline SAFETY PANMMTER & $\underset{\left|S_{1}\right|}{\operatorname{Fin})^{2}}$ & 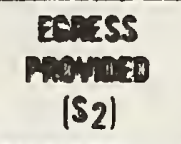 & 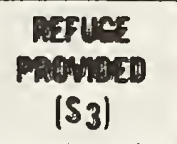 & $\begin{array}{l}\text { GEWERAL FIOE } \\
\text { SAFETY PROVIDED } \\
\text { (S4) }\end{array}$ \\
\hline 1. CONSTRuCtian & 2 & & 2 & 2 \\
\hline 2. HAZAMDOUS APEAS & 0 & $\div 2$ & 0 & 0 \\
\hline 3. MANUAL FIRE MLAM & $\div 2 \quad 1.5$ & 3 & & 3 \\
\hline $\begin{array}{l}\text { 4. SHOKE BETECTION \& } \\
\text { ALADIH }\end{array}$ & $\div 2 \quad 1$ & 2 & & 2 \\
\hline 5. AUTCAATIC SPMWmaLES & 2 & $\div 2(0) B_{B} \quad 1$ & $(\div 2) A_{1}$ & 2 \\
\hline $\begin{array}{l}\text { 6. WTERIDR FIMISH WITMM } \\
\text { LIVHG UNTS }\end{array}$ & -.5 & & & -1 \\
\hline $\begin{array}{l}\text { 7. LIVIAG UNIT - BDARA } \\
\text { DOORS \& OPEMUES }\end{array}$ & & & 0 & 0 \\
\hline $\begin{array}{l}\text { 8. EGRESS FROM LIVHAG } \\
\text { UNIT/S) }\end{array}$ & & -1 & & -1 \\
\hline $\begin{array}{l}\text { 9. SEPARATION OF LIVING } \\
\text { UNITS FROM EACH OTHER } \\
\text { \& FROM COMAON SPACES }\end{array}$ & 4 & $\div 2$ & 4 & 4 \\
\hline 10. EXIT SYSTEM & & 0 & $\div 2$ & 0 \\
\hline 11. EXIT ACCESS & & 0 & & 0 \\
\hline $\begin{array}{l}\text { 12. WTERIOR FIAISH } \\
\text { (egress routes| }\end{array}$ & & -1 & & -1 \\
\hline 13. VERTICAL OPEMHAS & $\div 2$ & 2 & 2 & 2 \\
\hline 14. SHOKE CONTROL & & 0 & 0 & 0 \\
\hline TOTAL. & $S_{1}=11$ & $s_{2}=$ & $S_{3}=$ & $S_{4}=$ \\
\hline \multicolumn{5}{|c|}{$\begin{array}{l}\text { NOTES: } \\
\text { A - Use full value if Safety Parameter } 1 \text { is based on } 2-U, 3-U \text { or } 4-U \text { construction. } \\
\text { Divide by } 2(\div 2) \text { in all other cases. } \\
\text { B - Use (0) if operation of sprinkler system does not activate building fire } \\
\text { alarm system. }\end{array}$} \\
\hline
\end{tabular}

Figure 15b. Mandatory Safety Requirements

Housing for the Elderly

$>6$ story $\leq 8$ units/floor 


\begin{tabular}{|c|c|c|c|c|c|c|c|c|c|c|c|c|}
\hline SAFETY PARAMETER & \multicolumn{11}{|c|}{ PARARTER VAUES } & \\
\hline \multirow{3}{*}{$\begin{array}{l}\text { 1. CONSIRUCTION } \\
\text { 8UILDHG HEIGHT } \\
\text { I-3 STORY }\end{array}$} & \multicolumn{8}{|c|}{ COMSUSTHEL } & \multicolumn{3}{|c|}{ DencennSTILLE } & \\
\hline & TYPE 4.U & IXas 4 & \multicolumn{2}{|c|}{ TYPE 3.U } & \multicolumn{2}{|c|}{ TYPES $3283 \mathrm{th}$} & \multicolumn{2}{|c|}{ TYPE ЗЕ НT. } & TYPE 2-U & TYPE $2 b$ & THPES 182 & \\
\hline & -2 & 0 & \multicolumn{2}{|c|}{-2} & \multicolumn{2}{|c|}{ (3) } & \multicolumn{2}{|l|}{-1} & -2 & 2 & 2 & \\
\hline 4-6 SIORY & -8 & -2 & \multicolumn{2}{|c|}{-8} & \multicolumn{2}{|c|}{0} & \multicolumn{2}{|l|}{-3} & -5 & 2 & 2 & \\
\hline OVER 6 STORY & -10 & -4 & \multicolumn{2}{|c|}{-10} & \multicolumn{2}{|c|}{-2} & \multicolumn{2}{|l|}{-4} & -8 & $\theta$ & 2 & \\
\hline \multirow{3}{*}{$\begin{array}{l}\text { 2. HAZARGOUS } \\
\text { AREAS }\end{array}$} & WITHIM LIVING & G UNITS O & ExI & REUTE & & & YTSWE LA & Ning u & Uants & mome on in & & \\
\hline & OQUBLE OEFICH & ENCY & E OE & ICIENCY & 0our & ME DCF & ICAEACY & SHAG & SLE OEFICIEN & & & \\
\hline & -7 & & -4 & & & $-4 \mid-7$ & & & $\theta$ & & & \\
\hline 3 MUIHA & HO ALARA & & & MaAUA & IL ACA & & & & & & & \\
\hline J. WI ARM & & $w / 0 F$ & ans & CATLON & & F.B. & THKICATHE & & & & & \\
\hline & -1 & & 2 & & & & 3 & & & & & \\
\hline & & SIMGLE & ON L & HNG UMT & is outy & & & HTE & ACOLUESTED & SYSTE & & \\
\hline 4. SMOKE DETECTION & NONE & Simcis & & cusay & IFYFI & & LWoine & 16 tem & MTS ency & & & \\
\hline & & Sing & & errent & & & VERY LEWEL & & EVERY BEE & rees & & \\
\hline & -4 & 0 & & 2 & & & 3 & & 4 & 6 & & \\
\hline 5. AUTOMAIIC & rowg & CORAIOER & vet I & SPACES. & ETC. & IW & UWTS OW: & & coneteans & m. \& Punc & SPACES & тотм еURеm. \\
\hline SPRINKLERS & 0 & & 2어바은 & & & & 4018 & & & 6 & & 3 \\
\hline 6. INTERHOR FINSH & & AHE SPRE & & & & & & & & & & \\
\hline (within living & $15=200$ & -25 & & $\leq 25$ & & & & & & & & \\
\hline แคits] & -3 & -1 & & 0 & & & & & & & & \\
\hline 7. LAMAG UATT - BOAM. & NO 000 R & $<20$ & & $\geq 28 \mathrm{~m}$ & & & & & & & & \\
\hline DOOAS \& OPENUAS & -2 & 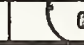 & & 1 & & & & & & & & \\
\hline & SAHGLE & E ROUTE & & 1000 & $2 \mathrm{ESC}$ & APE vim & eows & & EmIE & & & \\
\hline 8. EGRESS FROM & $=50^{\circ}$ TRAV & & & $-50^{\circ} \mathrm{TH}$ & & $\leq 50^{\circ}$ & TRAY. & & Exrs & & & \\
\hline & -2 & & & -1 & & 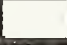 & $\theta$ & & 1 & & & \\
\hline 9. SEPARATIOAN OF & Mane On & & MLS & $-20 \mathrm{mat}$ & & & waLs $\geq 2$ & $\min$ & a $<1 \mathrm{~mm}$. & & & mus $\geq 2 \mathrm{~m}$. \\
\hline LIVING UHTS FROM & INCOMPLETE & 000RS & $\operatorname{mon}$ & Beons & $\geq 20$ & min. & & & & & & EMens $\geq 45$ mm. $w / A C$ \\
\hline FROA COM. SPACES & -6 & - & & & $|-2| A$ & & & $-2)$ & & $(-2)$ & & C \\
\hline & < 2 Stmaseno & & & IIPLE SI & Than & No newt & & & & & & \\
\hline 10. EXIT SYSTEM & ROUTES & Ogfortit & $w$ & o КеQ1z. & now & 12. EXIT & enect & EXIT & & & & \\
\hline & $-6 \mid 0\} E$ & $(213)$ & & 0 & & 2 & 4 & & & & & \\
\hline & & OEAO & & & & & & $m E$ & EAB ENO $>3$ & g' 8 Timat is: & & \\
\hline 11. EXIT ACCESS & $=160^{\circ}$ & 50.1 & & 35.50 & & $\rightarrow 1$ & $150^{\circ}$ & & $90-159$ & $50-160^{\circ}$ & $<50^{\circ}$ & \\
\hline & $-6|0| 0$ & -2 & & -1 & & - & & & -1 & $c$ & 2 & \\
\hline & & AaME SPAE & & & & & & & & & & \\
\hline [egress routes] & $-75 \leq 200$ & $7^{25}$ & & $\leq 25$ & & & & & & & & \\
\hline & -3 & & & 0 & & & & & & & & \\
\hline & & PEK ON M & PLET & Encles & & & & & EEcLOSEB & & & \\
\hline OPENHAS & THRU 4 OR MO & PAE FLGAR: & & FLES. & & $1 \mathrm{Fta}$ & & $1 \mathrm{~m}$. & & $\rightarrow$ & & \\
\hline & -10 & & & & & -2 & & $\theta$ & & $\because \sqrt{2}$ & & \\
\hline & me & & & Smone & & semen & E PRTI \& & & nectin. Assis & Fi) wroturs & & \\
\hline 14. SMOKE CONTROL & CONTROL & & & Towe & & Swen & E TEWER & & er zene & BY Lat/cenn. & & \\
\hline & 0 & & & 2 & & & 3 & & 3 & 4 & & \\
\hline
\end{tabular}

Figure 16a. MPS Requirements

Rehab. Multifamily Housing

$\leq 3$ story - > 8 units/floor 


\begin{tabular}{|c|c|c|c|c|}
\hline SAFETY PARAMETER & 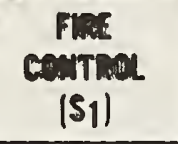 & $\begin{array}{l}\text { Echess } \\
\text { monact } \\
\left(S_{2}\right)\end{array}$ & 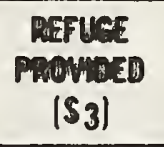 & $\begin{array}{l}\text { GEAEPAL FIPE } \\
\text { SAFETY PRBWAED } \\
\text { (S4) }\end{array}$ \\
\hline 1. CONSTRUCTIOA & 0 & & 0 & 0 \\
\hline 2. HAZMDOUS AREAS & 0 & $\div 2$ & 0 & 0 \\
\hline 3. MANUML FIRE MLAM & $\div 2 \quad 1$ & 2 & & 2 \\
\hline $\begin{array}{l}\text { 4. SMAKE DETECTIMA \& } \\
\text { ALARA }\end{array}$ & $\div 2 \quad 1$ & 2 & & 2 \\
\hline 5. AUTOMATIC SPAMmLLERS & 0 & $\div 2|0| B \quad 0$ & $\left(\div 2 \mid A_{0}\right.$ & 0 \\
\hline $\begin{array}{l}\text { 6. WTERIOR FIASH WTTHA } \\
\text { LIVANG UAHTS }\end{array}$ & $\div 2-1.5$ & & & -3 \\
\hline $\begin{array}{l}\text { 7. LIVING UNIT - BAIAM } \\
\text { DOORS \& OPEMHISS }\end{array}$ & & & 0 & 0 \\
\hline $\begin{array}{l}\text { 8. EGRESS FROM LIWMA } \\
\text { UNIT(S) }\end{array}$ & & -1 & & -1 \\
\hline $\begin{array}{l}\text { 9. SEPARATION OF LIVHAG } \\
\text { UNITS FROM EACH OTHER } \\
\text { \& FROA COMAOA SPACES }\end{array}$ & 4 & $\div 2$ & 4 & 4 \\
\hline 10. EXIT SYSTEM & & -2 & $\div 2-1$ & -2 \\
\hline 11. EXIT ACCESS & & -1 & & -1 \\
\hline $\begin{array}{l}\text { 12. WTERIOR FHASSH } \\
\text { |egress routes| }\end{array}$ & & -1 & & -1 \\
\hline 13. VERTICAL OPEAWUSS & $\div 2 \quad 1$ & 2 & 2 & 2 \\
\hline 14. SMOKE CONTROL & & 2 & 2 & 2 \\
\hline TOTAL. & $S_{1}=5.5$ & $S_{2}=5$ & $S_{3}=7$ & $S_{4}=$ \\
\hline $\begin{array}{l}\text { NOTES: } \\
\text { A - Use full value if Safet } \\
\text { Divide by } 2(\div 2) \text { in all } \\
\text { B - Use }(0) \text { if operation of } \\
\text { alarm system. }\end{array}$ & $\begin{array}{l}\text { Parameter } 1 \mathrm{i} \\
\text { her cases. } \\
\text { rinkler syste }\end{array}$ & $\begin{array}{l}\text { based on } 2-U \\
\text { does not ac }\end{array}$ & $\begin{array}{l}\text { 3-U or } 4-U \mathrm{C} \\
\text { ate building }\end{array}$ & $\begin{array}{l}\text { nstruction. } \\
\text { fire }\end{array}$ \\
\hline
\end{tabular}

Figure 16b. Mandatory Safety Requirements

Rehab. Multifamily Housing

$\leq 3$ story $->8$ units/floor 


\begin{tabular}{|c|c|c|c|c|c|c|c|c|c|c|}
\hline SAFETY PAMMATETER & \multicolumn{9}{|c|}{ PMONETER VALUES } & \\
\hline \multirow{3}{*}{$\begin{array}{l}\text { 1. CONSTRUCTEN } \\
\text { BULOHG HEIGHT } \\
\text { 1.3 STERY }\end{array}$} & \multicolumn{6}{|c|}{ comoustale } & \multicolumn{3}{|c|}{ MOHCEMUUSTIELE } & \\
\hline & TYPE 4.U & TYPE 4 & \multicolumn{2}{|c|}{ TYPE 3-U } & TYPES 3ab3b & TYPE $3 B$ НT. & TYPE 2-U & TYPE $2 b$ & TYPES 162a & \\
\hline & -2 & $\theta$ & \multicolumn{2}{|c|}{-2} & 息 & -1 & -2 & 2 & 2 & \\
\hline $4-6$ STERY & -8 & -2 & \multicolumn{2}{|c|}{-8} & $\theta$ & -3 & -5 & 2 & 2 & \\
\hline OWER G STOUY & -10 & -4 & -1 & & -2 & -4 & -8 & 0 & 2 & \\
\hline & WITHAN LIVMGG & G Units ar & Exir & HOUTE & & ITSIOE LWANG & Unts & NONE OR HC & & \\
\hline $\begin{array}{l}\text { 2. MatamiouUs } \\
\text { AfEAS }\end{array}$ & BOUGLE DEFICHE & \begin{tabular}{l|l} 
ENCY & $\mathrm{S}$ \\
\end{tabular} & If oft & chewer & Botente ACF & \begin{tabular}{l|l} 
crewar & SH \\
\end{tabular} & MGLE BEFICHEM & & & \\
\hline & -7 & & -4 & & $-4 \mid-7$ & & 0 & $0)$ & & \\
\hline & MD & & & mavidu & 14 ALAR A & & & & & \\
\hline ALARM & overem m & $w / 0 F$ & MeTIF & CATHE & $w / 5.0 / 6$ & mitcation & & & & \\
\hline & -1 & & 2 & & & 3) & & & & \\
\hline & & SIHCLE ST & $\mathrm{LH}$ & IWG UWTS & S EmLY & & IERCOWWECTED & SYSTEH & & \\
\hline 4. SHOKE DETECTION & NONE & SHAG & & & & LAVING U & MITS ONLY & & & \\
\hline & & SHGLE L & & EVERYL & [Evtht & IERY LEVEL & EVERY BEE & BULOH & & \\
\hline & -4 & $\theta$ & & 2 & ) & 3 & 4 & 6 & & \\
\hline 5. NUTBRATIC & Howe & cenericons & yeut & SPACES. E & เพพาด & UNITS ONLY & COARIBORS & S. HAB., \& PUBLICS & SPACES & TOTAL BUILOMG \\
\hline SPRHKLERS & 0 & & $2(\theta)$ & & & 4018 & & 6 & & 8 \\
\hline 6. HTERIOR FIMSH & & AME SPAEI & RATHAC & & & & & & & \\
\hline I within living & $-75=200$ & -25 & & $\leq 25$ & & & & & & \\
\hline & -3 & -1 & & 0 & & & & & & \\
\hline 7. LIVHG UNT - BBRA. & 1000009 & 20 & & $\geq 20 \mathrm{mHM}$ & & & & & & \\
\hline DOORS \& OPENHES & -2 & 0 & & 1 & & & & & & \\
\hline & SHGLE & E ROUTE & & $1000 R$ & 2 ESCAPE WHAC & oows & REMOTE & & & \\
\hline 8. EGRESS FROM & $=50^{\prime}$ TRAV & $=3$ & & $=50^{\circ}$ TRA & \begin{tabular}{l|l} 
RAV. & $\leq 50^{\circ}$ \\
\end{tabular} & IRAV & ExITs & & & \\
\hline & -2 & -1 & & -1 & $\theta$ & 9 & 1 & & & \\
\hline 9. SEPARATION OF & NONE OR & & MALS & -20 & & NALLS $\geq 20 \mathrm{~K}$ & $\operatorname{AMN}<1 \mathrm{HR}$ & WALLS $\geq 1 \mathrm{H}$ & & WALLS $\geq 2$ HR \\
\hline LIVIAG UAMTS FREM & IHCOMPLETE & Doons & $0 \mathrm{man}$ & Doons & $\geq 20$ ตัต & DOORS $\geq$ & $20 \min$ & $000 \mathrm{RS} \geq 20$ Mim & & OOORS $\geq 45 \mathrm{MIN} \sim \mathrm{AC}$ \\
\hline FROM COM. SPACES & -6 & - & & & $(-2) A$ & $21-2$ & & $(4-21)$ & & c \\
\hline & - 2 STANOARO & & & TIPLE STA & TANDARO ROUTE & & & & & \\
\hline 10. EXIT SYSTEM & ROUTES & OPflche & & O HORIZ. & HGRIZ EXIT & OHECT EXI & & & & \\
\hline & $-6 \mid$ 이 & 2101 & & 0 & 2 & 4 & & & & \\
\hline & & QEAD & & & & & DEAO END $>3$ & 35.8 TRAVEL IS & & \\
\hline 11. EXIT ACCESS & $-100^{\circ}$ & $50-16$ & & 25.50 & -15 & & $100 \cdot 150^{\circ}$ & $50-100^{\circ}$ & $=50^{\circ}$ & \\
\hline & $-6|0| 0$ & -2 & & -1 & -2 & & -1 & 0 & 2 & \\
\hline & & AHE SPREA & RATINC & & & & & & & \\
\hline 12. & $-75 \leq 200$ & 255 & & $\leq 25$ & & & & & & \\
\hline & -3 & -1 & & 0 & & & & & & \\
\hline & & EN OR IHC & APLET & ENCLOSUR & & & ENCLOSEO & & & \\
\hline OPENANGS & THRU 4 QR MOR & RE FLOORS & & FLRS. & $1 \mathrm{FLR}$. & $=1 \mathrm{H}$ & & $\mathrm{ThM}$ & & \\
\hline & -10 & & & 7 & -2 & 0 & & प101ब & & \\
\hline & no & smo: & & SMOKE PR & SMOKE & PART 1 & MECH. ASSIS & TEO AUTOMATIC & & \\
\hline 14. SMOKE CONTROL & CONTROL & & & TOWER & & E TOWER & BY ZONE & BY UNIT CORR. & & \\
\hline & 0 & & & 2 & & 3 & 3 & 4 & & \\
\hline
\end{tabular}

Figure 17a. NIPS Requirements

Rehab. Multifamily Housing

4-6 story - > 8 units/floor 


\begin{tabular}{|c|c|c|c|c|}
\hline SAFETY PARMMETER & $\begin{array}{l}\text { Fline } \\
\text { conthel } \\
{\left[S_{1}\right]}\end{array}$ & $\begin{array}{l}\text { EERESS } \\
\text { PIOMTED } \\
\text { [\$2] }\end{array}$ & $\begin{array}{l}\text { PaEFUGE } \\
\text { PREVHAED } \\
{\left[S_{3}\right]}\end{array}$ & $\begin{array}{l}\text { GENERAL FIRE } \\
\text { SAFETY PROWDED } \\
\text { (S4) }\end{array}$ \\
\hline 1. CONSTRUCTIOA & 0 & & 0 & 0 \\
\hline 2. HAZARDOUS AREAS & 0 & $\div 2$ & 0 & 0 \\
\hline 3. MAFUAL FIRE MLAM & $\div 2 \quad 1.5$ & 3 & & 3 \\
\hline $\begin{array}{l}\text { 4. SHOKE DETECTIMN \& } \\
\text { ALARM }\end{array}$ & $\div 2 \quad 1$ & 2 & & 2 \\
\hline 5. AUTOMATKC SPRMMLERS & 2 & $\div 2|0| B \quad 1$ & $(\div 2) \mid A$ & 2 \\
\hline $\begin{array}{l}\text { 6. HTERIOR FHASH WITHM } \\
\text { LIVIAG UNTS }\end{array}$ & $\div 2-1.5$ & & & -3 \\
\hline $\begin{array}{l}\text { 7. LIVING UNIT - BORM } \\
\text { DOORS \& OPEAMES }\end{array}$ & & & 0 & 0 \\
\hline $\begin{array}{l}\text { 8. EGRESS FROM LIVHG } \\
\text { UNIT(S) }\end{array}$ & & -1 & & -1 \\
\hline $\begin{array}{l}\text { 9. SEPARATION OF LIVIAG } \\
\text { UNITS FROM EACH OTHER } \\
\text { \& FROM COMMON SPACES }\end{array}$ & 4 & $\div 2$ & 4 & 4 \\
\hline 10. EXIT SYSTEM & & -2 & -1 & -2 \\
\hline 11. EXIT ACCESS & & -1 & & -1 \\
\hline $\begin{array}{l}\text { 12. MTEROR FWASH } \\
\text { (egress routes) }\end{array}$ & & -1 & & -1 \\
\hline 13. VERTICAL OPEAMAGS & $\div 2$ & 2 & 2 & 2 \\
\hline 14. SMOKE CONTROL & & 2 & 2 & 2 \\
\hline TOTAL & $S_{1}=8$ & $S_{2}=$ & $s_{3}=8$ & $S_{4}=$ \\
\hline \multicolumn{5}{|c|}{$\begin{array}{l}\text { NOTES: } \\
\text { A - Use full value if Safety Parameter } 1 \text { is based on } 2-U, 3-U \text { or } 4-U \text { construction. } \\
\text { Divide by } 2[\div 2] \text { in all other cases. } \\
\text { B - Use }[0] \text { if operation of sprinkler system does not activate building fire } \\
\text { alarm system. }\end{array}$} \\
\hline
\end{tabular}

Figure 17b. Mandatory Safety Requirements Rehab. Multifamily Housing 4-6 story - > 8 units/floor 


\begin{tabular}{|c|c|c|c|c|c|c|c|c|c|c|c|}
\hline SAFETY PMRMATER & \multicolumn{10}{|c|}{ PMAMETER VALUES } & \\
\hline \multirow{3}{*}{$\begin{array}{l}\text { 1. CENSTIUCTEN } \\
\text { BUH DHE HEIGHT } \\
\text { 1-3 STORY }\end{array}$} & \multicolumn{7}{|c|}{ comoustate } & \multicolumn{3}{|c|}{ MOACoHBUSTIBLE } & \\
\hline & TYPE 4.J & TYPE 4 & \multicolumn{2}{|c|}{ TYFE 3.4} & \multicolumn{2}{|c|}{ TYPES 3ak3 } & TYPE 3B MT. & TYPE 2.V & \multicolumn{2}{|r|}{ TYPES 162a } & \\
\hline & -2 & 0 & \multicolumn{2}{|c|}{-2} & \multicolumn{2}{|c|}{6} & -1 & -2 & 2 & 2 & \\
\hline 4.6 STERY & -8 & -2 & \multicolumn{2}{|c|}{-8} & \multicolumn{2}{|c|}{0} & -3 & -5 & 2 & 2 & \\
\hline OVER 6 STENY & -10 & -4 & \multicolumn{2}{|c|}{-10} & -2 & & -4 & -8 & 0 & 2 & \\
\hline & WITHAN LIVIAT & LWTS of & EXIT & ROUTE & & & JTSLE LWHA & Uaits & HONE OA $\mathrm{N}$ & & \\
\hline 2. HALAHEOUS & DofSA OEFICIE & ENCY & E BEF & ICHENCY & Dour & OLE BEF & \begin{tabular}{|l|l|} 
ICLENCY & SHAY \\
\end{tabular} & GE DEFICIEA & & & \\
\hline & -7 & & -4 & & & $-4 \mid-7)$ & & 0 & 0 & & \\
\hline & MO ALARH & & & MANUA & IL ALAR & & & & & & \\
\hline ALARM & & $w / 0 F$ & NIIFI & CATIOM & $w$ & F.D. & HELCATION & & & & \\
\hline & -1 & & 2 & & & & 3 & & & & \\
\hline & & SHELE ST & ON LH & ING UNAT & IS OAEY & & NTE & ERCONAECTED & SYSTEM & & \\
\hline 4. SMOKE DETECTION & MONE & & & & & & LAYMG UA & NITS OHLY & & & \\
\hline & & SIHGLE L & & EVERY & LEVEL & & VERY LEVL & EVERY BEO & EUILOI & DING & \\
\hline & -4 & 0 & & 2 & & & 3 & 4 & 6 & & \\
\hline 5. ANTEMATHC & MONE & Conerieon & ע日yc & SPACES. & ETC. & LHWAC & UNITS DHLY & CORRIDDRS & S. HAB., \& PUBLIC & SPACES & TOTAL BUILOING \\
\hline SPRHAXLERS & 0 & & $2|\theta| 8$ & & & & $4|0| B$ & & 6 & & 8 \\
\hline 6. WITERIOR FAUSH & & AME SPAE & & & & & & & & & \\
\hline Iwithin living & $=25-200$ & -25 & & $\leq 25$ & & & & & & & \\
\hline units] & $(-3)$ & -1 & & 0 & & & & & & & \\
\hline 7. LIVHG UAT - BORM. & HO DDOR & 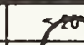 & & $\geq 20 \mathrm{M}$ & & & & & & & \\
\hline DOORS \& OPENINGS & -2 & & & 1 & & & & & & & \\
\hline & SHGLE & E ROUTE & & $1000 R$ & \& ESC & APE WHE & DDWS & REMDIE & & & \\
\hline $\begin{array}{l}\text { 8. FGRESS FROAN } \\
\text { INME UMTIS| }\end{array}$ & $>50^{\circ}$ TRAY & Fت & & $>50^{\circ} \mathrm{TR}$ & & $\leq 50^{\circ}$ & TRAY. & ExITS & & & \\
\hline & -2 & -1 & & -1 & & 8 & 0 & 1 & & & \\
\hline 9. SEPARATIOA OF & MONE OR & & ALLS & $>20 \mathrm{MHF}$ & & & WALLS $\geq 2 D$ MI & $\mathrm{IH} .=1 \mathrm{HR}$. & WALLS $\geq 1 \mathrm{H}$ & & WALLS $\geq 2$ MR \\
\hline LHANG UWTS FROM & INCDMPLETE & DOQRS & MIN. & DODRS & $s \geq 20$ & MHN. & OODRS $\geq 2$ & & ODORS $\geq 20 \mathrm{MH}$. & 1. $w A C$ & $000 R S \geq 45$ MIM \\
\hline EROM COM. SPACES & -6 & - & & & $(-2) A$ & & $2|-2|$ & & $(41-2)$ & & C \\
\hline & $=2$ STAMDARD & & & TIPLE ST & TANOAR & D ROUTE & & & & & \\
\hline 10. EXIT SYSTEM & ROUTES & OEELCH & & O HORIZ. & HDRI & 2. EXIT & DIRECT EXIT & & & & \\
\hline & $-6 ! 0 \mid E$ & $(-2 \mid \theta)$ & & 0 & & 2 & 4 & & & & \\
\hline & & OCAO & & & & & HD D & DEAD END $>3$ & $35^{\circ} \&$ TRAVEL IS & & \\
\hline 11. EXIT ACCESS & $=100^{\circ}$ & 50.1 & & 35.50 & & $>1$ & $50^{\circ}$ & \begin{tabular}{|l|l|}
$00 \cdot 150^{\circ}$ & \\
\end{tabular} & $50 \cdot 100^{\circ}$ & $<50^{\circ}$ & \\
\hline & $-6|0| B$ & -2 & & -1 & & -2 & & -1 & 0 & 2 & \\
\hline & & AME SPRE & ATINE & & & & & & & & \\
\hline 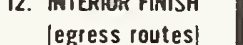 & $-75 \leq 200$ & 25 & & $\leq 25$ & & & & & & & \\
\hline & -3 & & & 0 & & & & & & & \\
\hline & & DEN OR INC & PLETE & EMCLDSL & & & & EMCLDSED & & & \\
\hline 13. VERTLAL & THRU 4 OR MOR & RE FLOORS & $2 \cdot 3$ & FLRS. & & FLR. & $<1 \mathrm{HR}$ & & Am & & \\
\hline & -10 & & - & 7 & & -2 & 0 & & 1015 & & \\
\hline & no & SMO & & SMOKE P & & SMOKE & PART. & MECH ASSIS & TED AUTOMATIC & & \\
\hline 14. SMOKE CONTROL & CDNTRDL & & & Тลพธ & & SMDKE & E TOWER & BY $20 \mathrm{HE}$ & BY UNIT, CORR. & & \\
\hline & 0 & & & 2 & & & 3 & 3 & 4 & & \\
\hline
\end{tabular}

Figure 18a. MPS Requirements

Rehab. Multifamily Housing

$>6$ story - > 8 units/floor 


\begin{tabular}{|c|c|c|c|c|c|}
\hline SAFETY PAMAMETER & $\begin{array}{l}\text { FHe } \\
\text { couttive } \\
{\left[S_{1} \mid\right.}\end{array}$ & $\begin{array}{l}\text { EERES } \\
\text { mavin: } \\
\left|S_{2}\right|\end{array}$ & & $\begin{array}{l}\text { PEFUCE } \\
\text { PROMDEED } \\
\text { [S } 3 \text { ] }\end{array}$ & $\begin{array}{l}\text { GENERAL FIRE } \\
\text { SAFETY PRQVIDED } \\
{\left[S_{4}\right]}\end{array}$ \\
\hline 1. constauctian & 2 & & & 2 & 2 \\
\hline 2. HAZMMEOUS ANEAS & 0 & $\div 2$ & 0 & 0 & 0 \\
\hline 3. MANUAL FWE MMAM & $\div 2 \quad 1.5$ & & 3 & & 3 \\
\hline $\begin{array}{l}\text { 4. SMOKE BETECTIBA \& } \\
\text { ALARA }\end{array}$ & $\div 2 \quad 1$ & & 2 & & 2 \\
\hline 5. AUTOMATKC SPRUAKLERS & 2 & $\div 2(0) B$ & 1 & $(\div 2) \mathrm{A} 1$ & 2 \\
\hline $\begin{array}{l}\text { 6. INTERIOR FHAISH WITLMN } \\
\text { LIVAG UNITS }\end{array}$ & $\div 2-1.5$ & & & & -3 \\
\hline $\begin{array}{l}\text { 7. LIVING UAT - BDAW } \\
\text { DOORS \& OPEMMES }\end{array}$ & & & & 0 & 0 \\
\hline $\begin{array}{l}\text { 8. EGRESS FRON LIVMAG } \\
\text { UNIT/S| }\end{array}$ & & & -1 & & -1 \\
\hline $\begin{array}{l}\text { 9. SEPARATION OF LIVING } \\
\text { UNITS FROM EACH OTHER } \\
\text { \& FROM COMMON SPACES }\end{array}$ & 4 & $\div 2$ & 2 & 4 & 4 \\
\hline 10. EXIT SYSTEM & & & -2 & $\div 2 \quad-1$ & -2 \\
\hline 11. EXIT ACCESS & & & -1 & & -1 \\
\hline $\begin{array}{l}\text { 12. INTERIOR FWNSH } \\
\text { (egress routes) }\end{array}$ & & & -1 & & -1 \\
\hline 13. VERTICAL OPENWAGS & $\div 2 \quad 1$ & & 2 & 2 & 2 \\
\hline 14. SMOKE CONTROL & & & 2 & 2 & 2 \\
\hline TOTAL & $S_{1}=10$ & $S_{2}=$ & 7 & $S_{3}=10$ & $S_{4}=$ \\
\hline $\begin{array}{l}\text { NOTES: } \\
\text { A - Use full value if Safet } \\
\text { Divide by } 2(\div 2) \text { in all } \\
\text { B - Use }(0) \text { if operation of } \\
\text { alarm system. }\end{array}$ & $\begin{array}{l}\text { Parameter } 1 \text { is } \\
\text { her cases. } \\
\text { rinkler syster }\end{array}$ & $\begin{array}{l}\text { based on } \\
\text { does ne }\end{array}$ & & $\begin{array}{l}3-U \text { or } 4-U \text { co } \\
\text { vate building }\end{array}$ & fire \\
\hline
\end{tabular}

\footnotetext{
Figure 18b. Mandatory Safety Requirements Rehab. Multifamily Housing $>6$ story - > 8 units/floor
} 


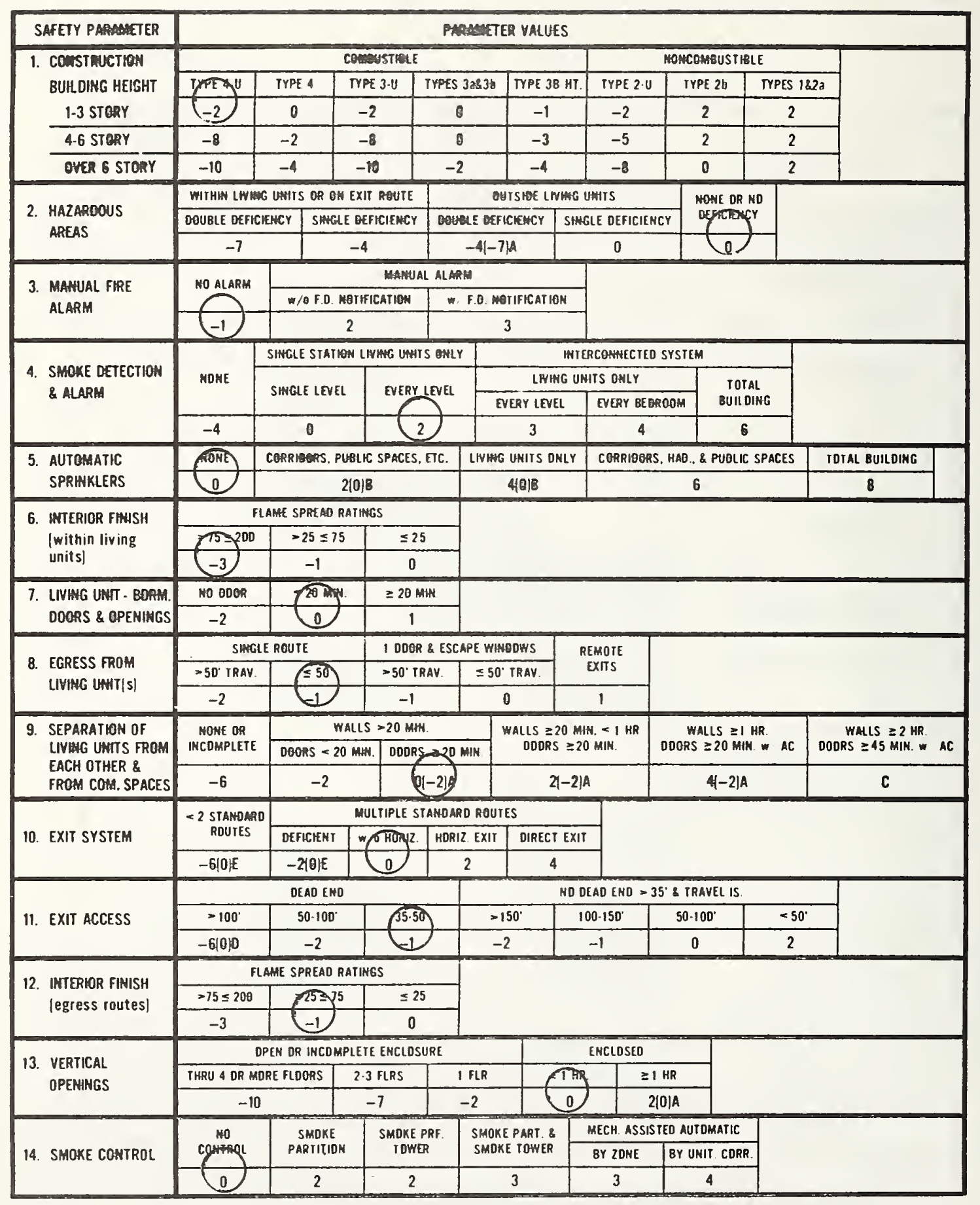

Figure 19a. MPS Requirements

Rehab. Multifamily Housing

1-2 story $-\leq 8$ units/floor 


\begin{tabular}{|c|c|c|c|c|c|}
\hline SAFETY PAANIETER & $\begin{array}{l}\text { Finim } \\
\text { contine } \\
\left(S_{1}\right)\end{array}$ & \multicolumn{2}{|c|}{$\begin{array}{l}\text { Eanss } \\
\text { Pewnozo } \\
\left(S_{2}\right)\end{array}$} & $\begin{array}{l}\text { REFLEE } \\
\text { PAOMEED } \\
\left(S_{3} \mid\right.\end{array}$ & $\begin{array}{l}\text { GEMERAL FHE } \\
\text { SAFETY PROVDED } \\
\text { (S4) }\end{array}$ \\
\hline 1. Comstauction & -2 & & & -2 & -2 \\
\hline 2. HAZMaguS ANEAS & 0 & $\div 2$ & 0 & 0 & 0 \\
\hline 3. MANEAL FITE MLAN & $\div 2-.5$ & & -1 & & -1 \\
\hline $\begin{array}{l}\text { 4. SHOXE DETECTIOA \& } \\
\text { ALAR }\end{array}$ & $\div 2 \quad 1$ & & 2 & & 2 \\
\hline 5. AUTOMATIC SPRAMULERS & 0 & $\div 2(0 ; B$ & & $(\div 2) A 0$ & 0 \\
\hline $\begin{array}{l}\text { 6. HTERIOR FHANSH WTIMM } \\
\text { LIVUAG UNTS }\end{array}$ & $\div 2-1.5$ & & & & -3 \\
\hline $\begin{array}{l}\text { 7. LIVIAG UWT - BORA } \\
\text { DOORS \& OPENHES }\end{array}$ & & & & 0 & 0 \\
\hline $\begin{array}{l}\text { 8. EGRESS FROA LIVHAG } \\
\text { UNIT(S) }\end{array}$ & & & -1 & & -1 \\
\hline $\begin{array}{l}\text { 9. SEPARATION OF LIVHIG } \\
\text { UNITS FROM EACH OTHER } \\
\text { \& FROM COMMON SPACES }\end{array}$ & -2 & $\div 2$ & -1 & -2 & -2 \\
\hline 10. EXIT SYSTEM & & & 0 & $\div 2$ & 0 \\
\hline 11. EXIT ACCESS & & & -1 & & -1 \\
\hline $\begin{array}{l}\text { 12. INTEROR FINISH } \\
\text { (egress routes) }\end{array}$ & & & -1 & & -1 \\
\hline 13. VERTICAL OPENHASS & $\div 20$ & & 0 & 0 & 0 \\
\hline 14. SHOKE CONTROL & & & 0 & 0 & 0 \\
\hline TOTAL & $S_{1}=-5$ & $S_{2}=$ & -3 & $S_{3}=-4$ & $S_{4}=$ \\
\hline $\begin{array}{l}\text { NOTES: } \\
\text { A - Use full value if Safet } \\
\text { Divide by } 2(\div 2) \text { in all } \\
\text { B - Use }(0) \text { if operation of } \\
\text { alarm system. }\end{array}$ & $\begin{array}{l}\text { Parameter } 1 \text { i } \\
\text { her cases. } \\
\text { rinkler syste }\end{array}$ & $\begin{array}{l}\text { based } 0 \\
\text { does } n\end{array}$ & $2 \cdot U$ & $\begin{array}{l}3-\mathrm{U} \text { or } 4-\mathrm{U} \mathrm{CC} \\
\text { vate building }\end{array}$ & istruction. \\
\hline
\end{tabular}

Figure 19b. Mandatory Safety Requirements Rehab. Multifamily Housing 1-2 story - $\leq 8$ units/floor 


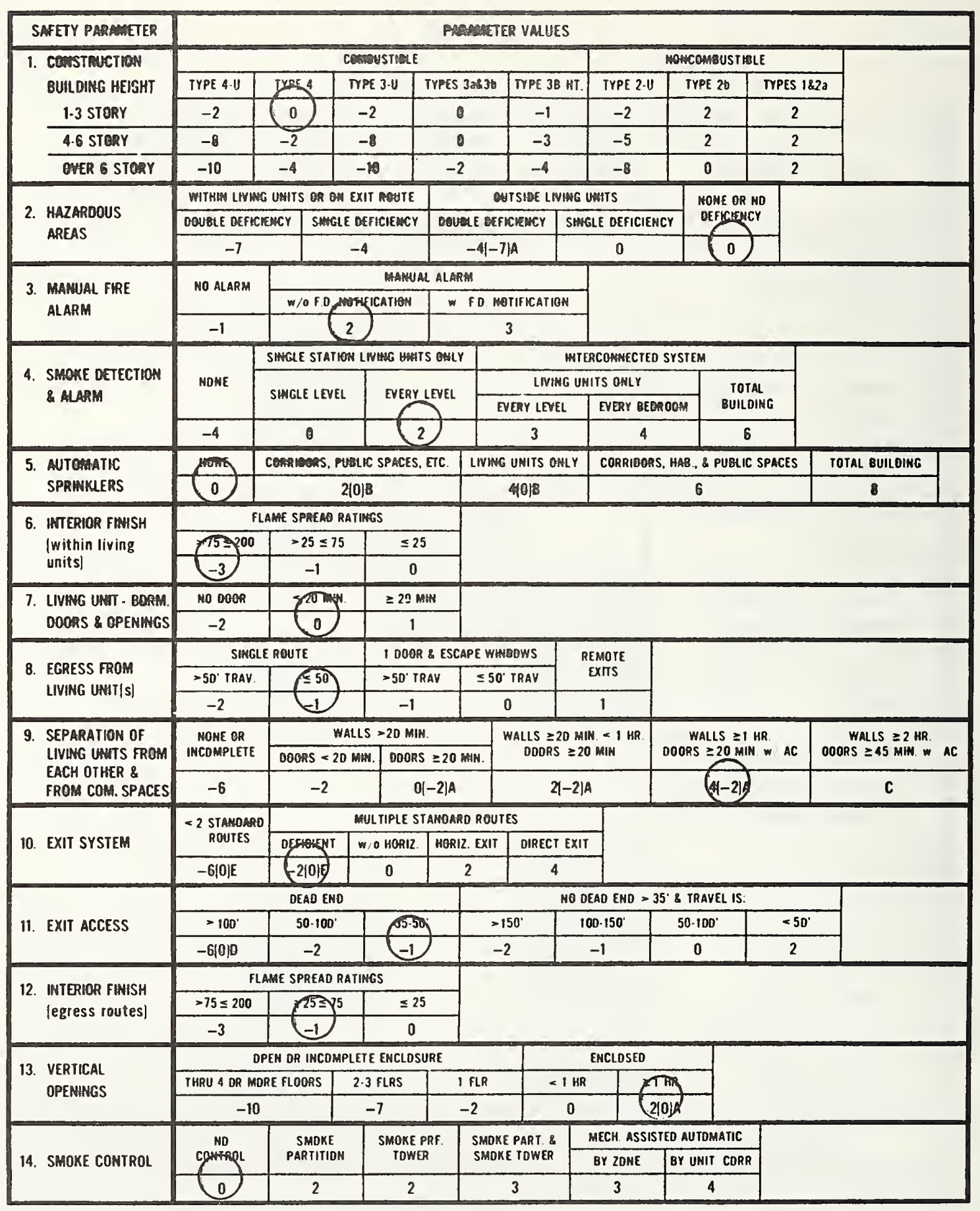

Figure 20a. MPS Requirements

Rehab. Multifamily Housing

3 story $-\leq 8$ units/floor 


\begin{tabular}{|c|c|c|c|c|c|}
\hline SAFETY PAMMIIETER & $\begin{array}{l}\text { Fing: } \\
\text { (S1) }\end{array}$ & 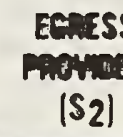 & & $\begin{array}{l}\text { REFUEE } \\
\text { PROHUED } \\
\text { (S3) }\end{array}$ & $\begin{array}{l}\text { GENERAL FHEE } \\
\text { SAFETY PROVDEE } \\
\text { (S4) }\end{array}$ \\
\hline 1. ConstRuctine & 0 & & & 0 & 0 \\
\hline 2. HAZMEams MEAS & 0 & $\div 2$ & 0 & 0 & 0 \\
\hline 3. MANUAL FIAE MAM & $\div 2 \quad I$ & & 2 & & 2 \\
\hline $\begin{array}{l}\text { 4. SMBAE BETECTIAH \& } \\
\text { ALAM }\end{array}$ & $\div 2 \quad 1$ & & 2 & & 2 \\
\hline 5. AUTOMATIC SFMmLLERS & 0 & $\div 2(0) B$ & 0 & {$[\div 2] \mathrm{A}_{0}$} & 0 \\
\hline $\begin{array}{l}\text { 6. WTERIOR FIMASH WMTIM } \\
\text { LIVHA UNTS }\end{array}$ & $\div 2-1.5$ & & & & -3 \\
\hline $\begin{array}{l}\text { 7. LIVHA UNT - BDPHA } \\
\text { DOORS \& OPEHUES }\end{array}$ & & & & 0 & 0 \\
\hline $\begin{array}{l}\text { 8. EGRESS FROA LIVHA } \\
\text { UAHT|S| }\end{array}$ & & & -1 & & -1 \\
\hline $\begin{array}{l}\text { 9. SEPARATHON OF LIMIM } \\
\text { UNITS FROM EACH OTHER } \\
\text { \& FROM COMHON SPACES }\end{array}$ & 4 & $\div 2$ & 2 & 4 & 4 \\
\hline 10. EXIT SYSTEMA & & & -2 & -1 & -2 \\
\hline 11. EXIT ACCESS & & & -1 & & -1 \\
\hline $\begin{array}{l}\text { 12. INTERIOR FINISH } \\
\text { (egress routes) }\end{array}$ & & & -1 & & -1 \\
\hline 13. VERTICAL OPEAHAGS & $\div 2 \quad 1$ & & 2 & 2 & 2 \\
\hline 14. SMOKE CONTROL & & & 0 & 0 & 0 \\
\hline TOTAL & $S_{1}=5.5$ & $S_{2}=$ & 3 & $S_{3}=$ & $S_{4}=$ \\
\hline \multicolumn{6}{|c|}{$\begin{array}{l}\text { A - Use full value if Safety Parameter } 1 \text { is based on } 2-U, 3-U \text { or } 4-U \text { construction. } \\
\text { Divide by } 2(\div 2) \text { in all other cases. } \\
\text { B - Use (0) if operation of sprinkler system does not activate building fire }\end{array}$} \\
\hline
\end{tabular}

Figure 20b. Mandatory Safety Requirements Rehab. Multifamily Housing 3 story $-\leq 8$ units/floor 


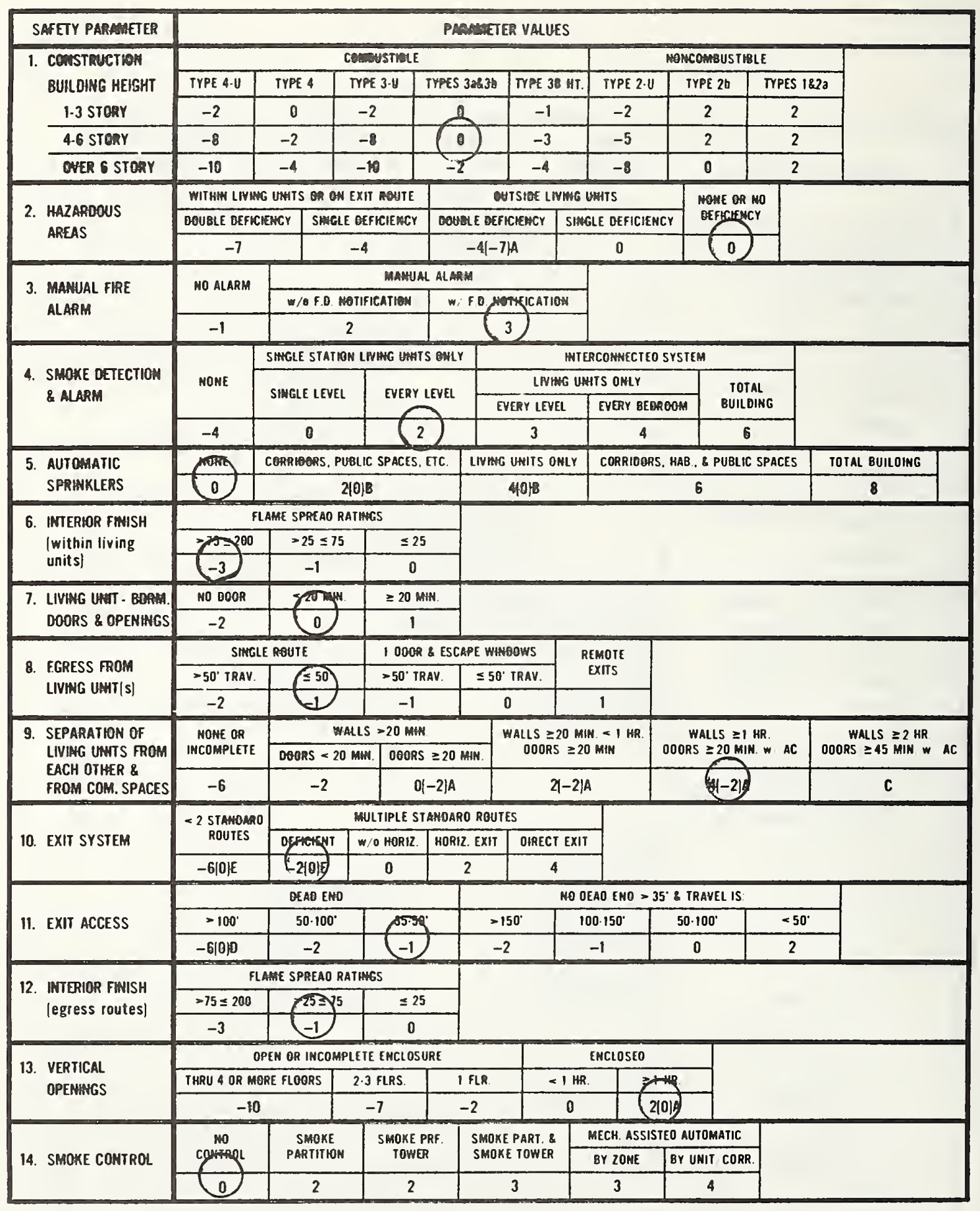

Figure 2la. MPS Requirements

Rehab. Multifamily Housing

4-6 story $-\leq 8$ units/floor 


\begin{tabular}{|c|c|c|c|c|}
\hline SAFETY PAMAMETER & $\underset{\text { Coimitine }}{\text { Pint }}$ & 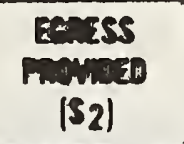 & 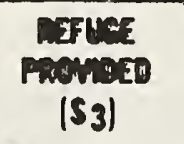 & $\begin{array}{l}\text { GEAERA FIAE } \\
\text { SAFETY PROWDEB } \\
\text { (S4) }\end{array}$ \\
\hline 1. constmetray & 0 & & 0 & 0 \\
\hline 2. HZZMans MNAS & 0 & $\div 2$ & 0 & 0 \\
\hline 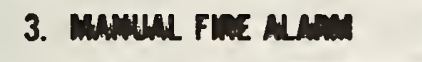 & $\div 2 \quad 1.5$ & 3 & & 3 \\
\hline $\begin{array}{l}\text { 4. SUAKE BETECTIN \& } \\
\text { ALANM }\end{array}$ & $\div 2 \quad 1$ & 2 & & 2 \\
\hline 5. AUTGMATIC SFrmmLERS & 0 & $\div 2(0) B \quad 0$ & $(\div 2) A 0$ & 0 \\
\hline 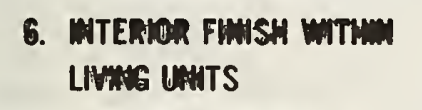 & $\div 2-1.5$ & & & -3 \\
\hline 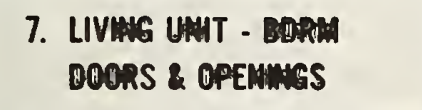 & & & 0 & 0 \\
\hline $\begin{array}{l}\text { 8. EGPESS FROM LIYMA } \\
\text { UAHT|S| }\end{array}$ & & -1 & & -1 \\
\hline $\begin{array}{l}\text { 9. SEPARATION OF LIVIHG } \\
\text { UAITS FROM EMCH OTHER } \\
\text { \& FROA COMMOA SPACES }\end{array}$ & 4 & $\div 2$ & 4 & 4 \\
\hline 10. EXIT SYSTEMA & & -2 & $\div 2$ & -2 \\
\hline 11. EXIT ACCESS & & -1 & & -1 \\
\hline $\begin{array}{l}\text { 12. HTERAQR FMASH } \\
\text { (egress routes) }\end{array}$ & & -1 & & -1 \\
\hline 13. VERTICM OPEMHES & $\div 2 \quad 1$ & 2 & 2 & 2 \\
\hline 14. SMOKE CONTROL & & 0 & 0 & 0 \\
\hline TOTAL & $S_{1}=6$ & $S_{2}=4$ & $S_{3}=$ & $S_{4}=$ \\
\hline $\begin{array}{l}\text { NOTES: } \\
\text { A - Use full value if Safe } \\
\text { Divide by } 2(\div 2) \text { in al } \\
\text { B - Use (0) if operation of } \\
\text { alarm system. }\end{array}$ & $\begin{array}{l}\text { arameter } 1 \text { is } \\
\text { ler cases. } \\
\text { rinkler syste }\end{array}$ & does not act & rate building & istruction. \\
\hline
\end{tabular}

Figure 21b. Mandatory Safety Requirements

Rehab. Multifamily Housing

4-6 story $-\leq 8$ units/floor 


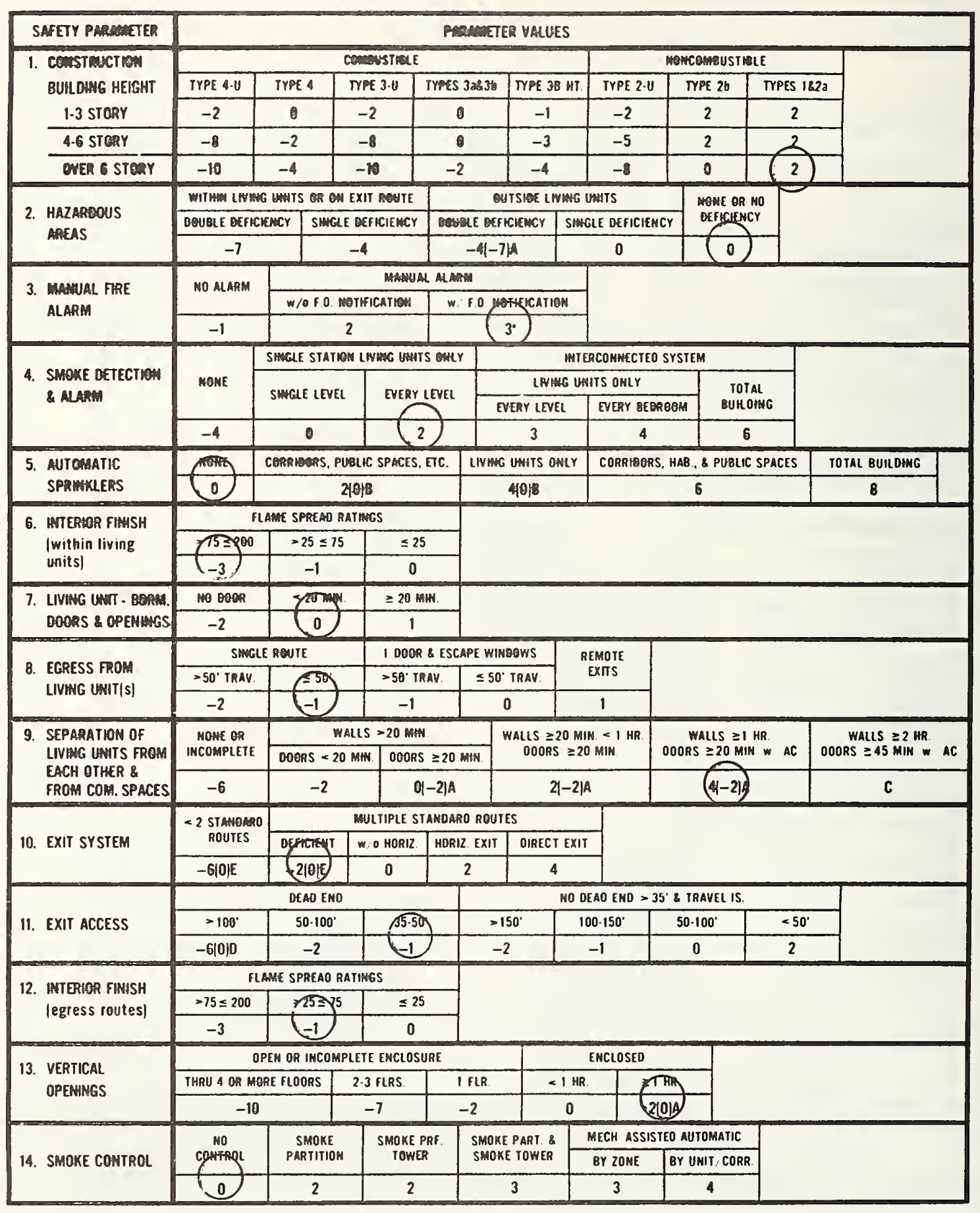

Figure 22a. MPS Requirements

Rehab. Multifamily Housing

$>6$ story $-\leq 8$ units/floor 


\begin{tabular}{|c|c|c|c|c|c|}
\hline SAFETY PARAmETER & $\begin{array}{l}\text { Fime } \\
\text { contmal } \\
\left(S_{1}\right)\end{array}$ & $\begin{array}{l}\text { EAmess } \\
\text { Proming } \\
\text { (S2) }\end{array}$ & & $\begin{array}{l}\text { REFUEE } \\
\text { PAOMAED } \\
\left(S_{3}\right)\end{array}$ & $\begin{array}{l}\text { GEMERM FIRE } \\
\text { SAFETY PRONIOED } \\
\text { (S4) }\end{array}$ \\
\hline 1. construction & 2 & & & 2 & 2 \\
\hline 2. Hazimanus mEEAS & 0 & $\div 2$ & 0 & 0 & 0 \\
\hline 3. MAMLAL FIRE MLPM & $\div 2 \quad 1.5$ & & 3 & & 3 \\
\hline $\begin{array}{l}\text { 4. SHOXE DETECTIZN \& } \\
\text { ALAMA }\end{array}$ & $\div 2 \quad 1$ & & 2 & & 2 \\
\hline 5. AUTOMATIC SFmiKLERS & 0 & $\div 2|0| B$ & 0 & $(\div 2) \mathrm{A} 0$ & 0 \\
\hline $\begin{array}{l}\text { 6. WTERAOR FINISH WTIMN } \\
\text { LIVING UNTS }\end{array}$ & $\div 2-1.5$ & & & & -3 \\
\hline $\begin{array}{l}\text { 7. LIVIMG UAT - BQRM } \\
\text { DOORS \& OPENMGS }\end{array}$ & & & & 0 & 0 \\
\hline $\begin{array}{l}\text { 8. EGRESS FROA LIVMG } \\
\text { UAHT(S) }\end{array}$ & & & -1 & & -1 \\
\hline $\begin{array}{l}\text { 9. SEPARATION OF LIVIAG } \\
\text { UNITS FROM EACH OTHER } \\
\text { \& FROM COMAON SPACES }\end{array}$ & 4 & $\div 2$ & 2 & 4 & 4 \\
\hline 10. EXIT SYSTEM & & & -2 & $\div 2 \quad-1$ & -2 \\
\hline 11. EXIT ACCESS & & & -1 & & -1 \\
\hline $\begin{array}{l}\text { 12. WTERHOR FMISH } \\
\text { (egresS routes) }\end{array}$ & & & -1 & & -1 \\
\hline 13. VERTICAL OPENHGS & $\div 2 \quad 1$ & & 2 & 2 & 2 \\
\hline 14. SHOKE CONTRQL & & & 0 & 0 & 0 \\
\hline TOTAL & $S_{1}=8$ & $S_{2}=$ & 4 & $S_{3}=\%$ & $S_{4}=$ \\
\hline $\begin{array}{l}\text { NOTES: } \\
\text { A - Use full value if Safet } \\
\text { Divide by } 2(\div 2) \text { in all } \\
\text { B - Use }(0) \text { if operation of } \\
\text { alarm system. }\end{array}$ & $\begin{array}{l}\text { arameter } 1 \text { i } \\
\text { her cases. } \\
\text { rinkler syste }\end{array}$ & $\begin{array}{l}\text { based on } \\
\text { does not }\end{array}$ & & ate building & istruction. \\
\hline
\end{tabular}

Figure 22b. Mandatory Safety Requirements

Rehab. Multifamily Housing

$>6$ story $-\leq 8$ units/floor 


\begin{tabular}{|c|c|c|c|c|c|c|c|c|c|c|c|}
\hline SAFETY PAMAMETER & \multicolumn{10}{|c|}{ PARMAETER YALUES } & \\
\hline \multirow{3}{*}{$\begin{array}{l}\text { 1. CONSTRUCTIN } \\
\text { BUILOAG HEIGHT } \\
\text { 1-3 STEAY }\end{array}$} & \multicolumn{7}{|c|}{ comoustuale } & \multicolumn{3}{|c|}{ HONCOABUSTHBLE } & \\
\hline & TYPE 4-U & $27+5$ & \multicolumn{2}{|c|}{ TYPE 3.H } & \multicolumn{2}{|c|}{ TFFES 32t3t } & TYFE $38 \mathrm{HT}$. & TYPE 2.U & TYPE $2 \mathrm{~h}$ & TYPES 182a & \\
\hline & -2 & (0) & \multicolumn{2}{|c|}{-2} & \multicolumn{2}{|l|}{0} & -1 & -2 & 2 & 2 & \\
\hline 4.6 STear & -8 & -2 & \multicolumn{2}{|c|}{-8} & 0 & & -3 & -5 & 2 & 2 & \\
\hline ONER S STENY & -10 & -4 & -6 & & -2 & & -4 & -8 & 0 & 2 & \\
\hline & WITHA LNWG & Units 8 & ExוT & ReyTE & & & TSHEE LHWW & 86 youts & MOME OR $\mathrm{K}$ & & \\
\hline $\begin{array}{l}\text { 2. HAZARLLOUS } \\
\text { AREAS }\end{array}$ & Devete BefiCiel & EMCY & IE BEFI & EHEWY & Bevele & DEFic & Cuswcy & HWLLE DEFICIEN & 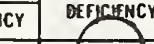 & & \\
\hline & -7 & & -4 & & & $4-74$ & & $\theta$ & 0 & & \\
\hline 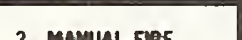 & 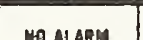 & & & Mamal & A MARA & & & & & & \\
\hline 3. WARM & 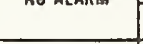 & $w / 0$ & HOFIF & CATEM & $w / F$ & 9. $\mathrm{mit}$ & TIFICATHON & & & & \\
\hline & -1 & & $2)$ & & & 3 & & & & & \\
\hline & & SWEG $S$ & ON LN & แats & $s$ enty & & & НTERCONWECTED & SYSTEM & & \\
\hline 4. SMOKE BETECTION & MONE & SHEAE I & & EYERY LE & LFYFI & & LAIMG & UNITS ONLY & & & \\
\hline & & & & EVERY LE & & & ERY LEVL & EVRY BED & BUHLOH & & \\
\hline & -4 & 0 & & 2 & ) & & 3 & 4 & 6 & & \\
\hline 5. AUTGMATIC & rong & comater & vetic & SPACES, E & ETC. & What 1 & UWITS OMLY & CORRIOOAS & S, MAB., \& PUBLIC & SPACES & TOTAL BUILONG \\
\hline SPRWKKLERS & $(0)$ & & $2\langle 0| B$ & & & & 4018 & & 6 & & 8 \\
\hline 6. NTERLOR FWHSH & & AAE SPRE & RATWE & & & & & & & & \\
\hline I within living & $-75 \leq 200$ & $\sqrt{25}$ & & $\leq 25$ & & & & & & & \\
\hline & -3 & 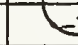 & & 0 & & & & & & & \\
\hline 7. LIYUGG UNTI - BERA. & 1100008 & $\sqrt{20}$ & & $\geq 20 \mathrm{~mm}$ & & & & & & & \\
\hline DOORS \& OPENHGS & -2 & 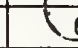 & & 1 & & & & & & & \\
\hline & SHGLE & E REUTE & & 1000 \& 8 & 8. ESCAPE & E WINO & bows & REMOTE & & & \\
\hline 8. ECRESS FROM & $=50^{\circ}$ TRAV & $E$ & & $=59^{\circ} \mathrm{TRA}$ & & $\leq 50^{\circ} 1$ & TRAV. & Extrs & & & \\
\hline & -2 & & & -1 & & 0 & & 1 & & & \\
\hline 9. SEPAAATION OF & MONE OR & & HALLS & -20 MN. & & & YALLS $\geq 20$ & MIN $<1$ MR. & WALLS $\geq 1 \mathrm{H}$ & & WALLS $\geq 2 \mathrm{HR}$. \\
\hline LIVHG UATS FROM & INCOMPLETE & De00RS & $0 \mathrm{ntw}$. & BCORS: & $\geq 20 \mathrm{MIF}$ & & DDOPS = $=2$ & $=20$ MIN & DOORS $\geq 20 \mathrm{MiN}$ & & DOORS $\geq 45$ MiK. $w, A C$ \\
\hline FROH COH. SPACES & -6 & & & & $-2 \mid A$ & & $x-2$ & & $(4-216)$ & & C \\
\hline & = 2 STAMOARO & & & TIPLE STA & ANDARD R & ROUTES & & & & & \\
\hline 10. EXIT SYSTEM & ROUTES & Oefriet & & HORIZ. & HOR12 E & EXIT & OHRECT EX & & & & \\
\hline & $-6|0| E$ & -210 & & 0 & 2 & & 4 & & & & \\
\hline & & DEAB & & & & & & D DEAD END > 3 & $35^{\prime}$ \& TRAVEL IS: & & \\
\hline 11. EXIT ACCESS & $=100^{\circ}$ & 50 . & & As.sp: & & -15 & & $100 \cdot 150^{\circ}$ & $50 \cdot 100^{\circ}$ & $=50^{\circ}$ & \\
\hline & $-6 / 010$ & - & & $(-1)$ & & -2 & & -1 & 0 & 2 & \\
\hline 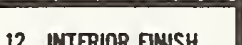 & & AAF SPRE & RATIMG & & & & & & & & \\
\hline $\begin{array}{l}\text { 12. Antess routes] } \\
\text { [egress fins }\end{array}$ & $-75 \leq 200$ & 25 & & $\leq 25$ & & & & & & & \\
\hline & -3 & - & & 0 & & & & & & & \\
\hline & & DEN OR IN & IPIETE & ENCLOSUR & & & & ENCLOSED & & & \\
\hline $\begin{array}{l}\text { 13. VRENALAL } \\
\text { OPENAMGS }\end{array}$ & THRU 4 OR MOR & RE FLOOR & $2-3$ & FLRS. & $1 \mathrm{FL}$ & & -11 & \begin{tabular}{l|l} 
HR. & 31 \\
\end{tabular} & & & \\
\hline & -10 & & - & 7 & -2 & & 0 & & 1010) & & \\
\hline & No & & & SHOKE PR & & SMOKE & PART. \& & MECH. ASSIS & TED AUTOMATIC & & \\
\hline 14. SMOKE COHTROL & COHTROL & & & TOWER & & SMOKE & TOWER & BY ZDNE & BY UNIT/CORR. & & \\
\hline & 0 & 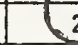 & & 2 & & & 3 & 3 & 4 & & \\
\hline
\end{tabular}

Figure 23a. MPS Requirements

Rehab. Housing for the Elderly

$\leq 3$ story - > 8 units/floor 


\begin{tabular}{|c|c|c|c|c|}
\hline SAFETY PAPMITER & $\begin{array}{l}\text { Finiz } \\
\text { ceutreal } \\
\left|S_{1}\right|\end{array}$ & 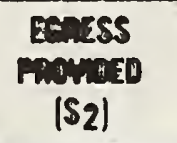 & $\begin{array}{l}\text { PEFUEF } \\
\text { PRANAED } \\
\text { [\$3] }\end{array}$ & $\begin{array}{l}\text { GENERAL FIEE } \\
\text { SAFETY PROMDED } \\
\left(\mathrm{S}_{4}\right)\end{array}$ \\
\hline 1. constanctian & $\dot{0}$ & & 0 & 0 \\
\hline 2. HAZMODOS MREAS & 0 & $\div 2$ & 0 & 0 \\
\hline 3. MUMUL FINE MUN & $\div 21$ & 2 & & 2 \\
\hline 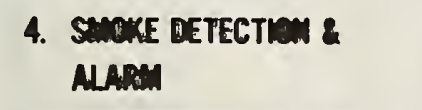 & $\div 2 \quad 1$ & 2 & & 2 \\
\hline 5. AUTOMATIC SFammLERS & 0 & $\div 2|\theta| B \quad 0$ & $(\div 2) \mathrm{A} 0$ & 0 \\
\hline 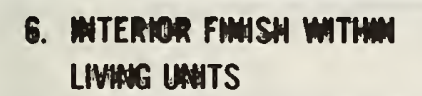 & $\div 2-.5$ & & & -1 \\
\hline $\begin{array}{l}\text { 7. LIVHE LANT - BORM } \\
\text { DOORS \& OPEAWAS }\end{array}$ & & & 0 & 0 \\
\hline $\begin{array}{l}\text { 8. EGRESS FROM LIMUG } \\
\text { UNWT(S) }\end{array}$ & & -1 & & -1 \\
\hline $\begin{array}{l}\text { 9. SEPARATION OF LIVING } \\
\text { UNITS FROM EACH OTHER } \\
\text { \& FROM COMMON SPACES }\end{array}$ & 4 & $\div 2$ & 4 & 4 \\
\hline 10. EXIT SYSTEM & & -2 & $\div 2 \quad-1$ & -2 \\
\hline 11. EXIT ACCESS & & -1 & & -1 \\
\hline $\begin{array}{l}\text { 12. INTEROR FMASH } \\
\text { (egress routes) }\end{array}$ & & -1 & & -1 \\
\hline 13. VERTICAL OPENMES & $\div 2$ & 2 & 2 & 2 \\
\hline 14. SMOKE CONTROL & & 2 & 2 & 2 \\
\hline TOTAL & $S_{1}=6.5$ & $S_{2}=$ & $S_{3}=$ & $S_{4}=$ \\
\hline $\begin{array}{l}\text { NOTES: } \\
\text { A - Use full value if Safe } \\
\text { Divide by } 2(\div 2) \text { in all } \\
\text { B - Use }(0) \text { if operation of } \\
\text { alarm system. }\end{array}$ & $\begin{array}{l}\text { arameter } 1 \text { is } \\
\text { jer cases. } \\
\text { rinkler syster }\end{array}$ & based on 2-U & late building & $\begin{array}{l}\text { struction. } \\
\text { ire }\end{array}$ \\
\hline
\end{tabular}

Figure 23b. Mandatory Safety Requirements Rehab. Housing for the Elderly $\leq 3$ story - > 8 units/floor 


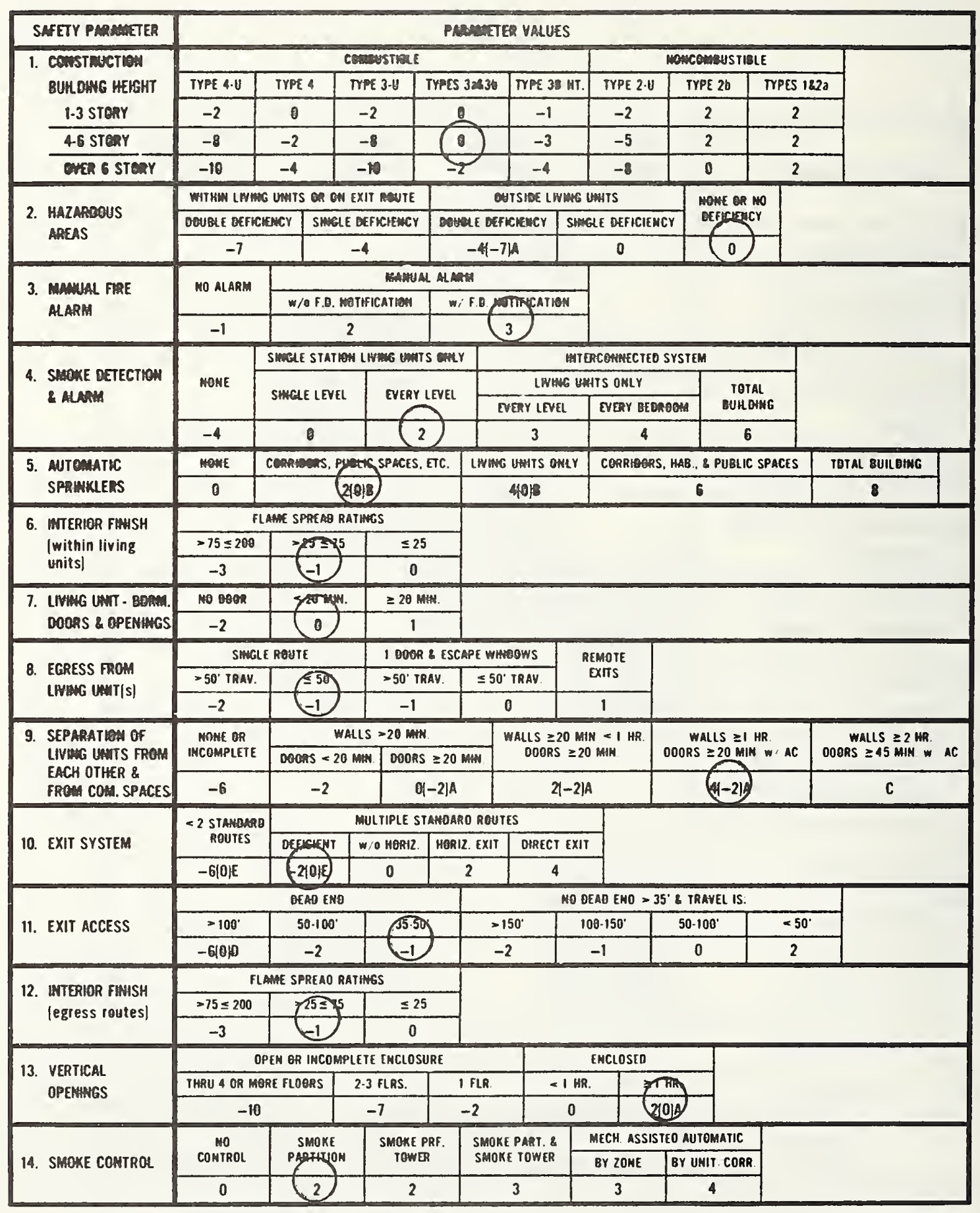

Figure 24a. MPS Requirements

Rehab. Housing for the Elderly

4-6 story - > 8 units/floor 


\begin{tabular}{|c|c|c|c|c|}
\hline SAFETY PAMAMETER & $\begin{array}{l}\text { Fimiz } \\
\text { Caitimil } \\
{\left[S_{1}\right]}\end{array}$ & 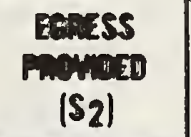 & 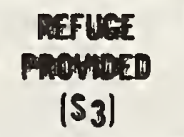 & $\begin{array}{l}\text { GENERAL FIOE } \\
\text { SAFETY PROVIDED } \\
\text { (S4) }\end{array}$ \\
\hline 1. Constauction & 0 & & 0 & 0 \\
\hline 2. HAZMEBOUS ANEAS & 0 & $\div 2$ & 0 & 0 \\
\hline 3. МАMUML FIAE MAMM & $\div 2 \quad 1.5$ & 3 & & 3 \\
\hline $\begin{array}{l}\text { 4. SMERE BETECTIAN \& } \\
\text { ALAMM }\end{array}$ & $\div 2 \quad 1$ & 2 & & 2 \\
\hline 5. AUTOAMATC SFOMUKLERS & 2 & $\div 2|0|$ ojB $\quad 1$ & $(\div 2) A$ & 2 \\
\hline $\begin{array}{l}\text { 6. WTERLER FWWSH WTHM } \\
\text { LIMANG LMTS }\end{array}$ & $\div 2-.5$ & & & -1 \\
\hline $\begin{array}{l}\text { 7. LIVHG UNIT - BDPA } \\
\text { DOORS \& OPEALASS }\end{array}$ & & & 0 & 0 \\
\hline $\begin{array}{l}\text { 8. EGRESS FROH LIVIING } \\
\text { UNIT/S) }\end{array}$ & & -1 & & -1 \\
\hline $\begin{array}{l}\text { 9. SEPARATION OF LIVIG } \\
\text { UNITS FROM EACH OTHER } \\
\text { \& FROM COMMOM SPACES }\end{array}$ & 4 & $\div 2$ & 4 & 4 \\
\hline 10. EXIT SYSTEM & & -2 & $\div 2 \quad-1$ & -2 \\
\hline 11. EXIT ACCESS & & $-\dot{1}$ & & -1 \\
\hline $\begin{array}{l}\text { 12. INTEROR FWWSH } \\
\text { (egresS routes) }\end{array}$ & & -1 & & -1 \\
\hline 13. VERTICA OPEAMHSS & $\div 2$ & 2 & 2 & 2 \\
\hline 14. SMOKE CONTROL & & 2 & 2 & 2 \\
\hline TOTAL & $S_{1}=$ & $S_{2}=$ & $s_{3}=$ & $S_{4}=$ \\
\hline \multicolumn{5}{|c|}{$\begin{array}{l}\text { NOTES: } \\
\text { A - Use full value if Safety Parameter } 1 \text { is based on } 2-U, 3-U \text { or } 4-U \text { construction. } \\
\text { Divide by } 2(\div 2) \text { in all other cases. } \\
\text { B - Use }(0) \text { if operation of sprinkler system does not activate building fire }\end{array}$} \\
\hline
\end{tabular}

Figure 24b. Mandatory Safety Requirements Rehab. Housing for the Elderly 4-6 story - > 8 units/floor 


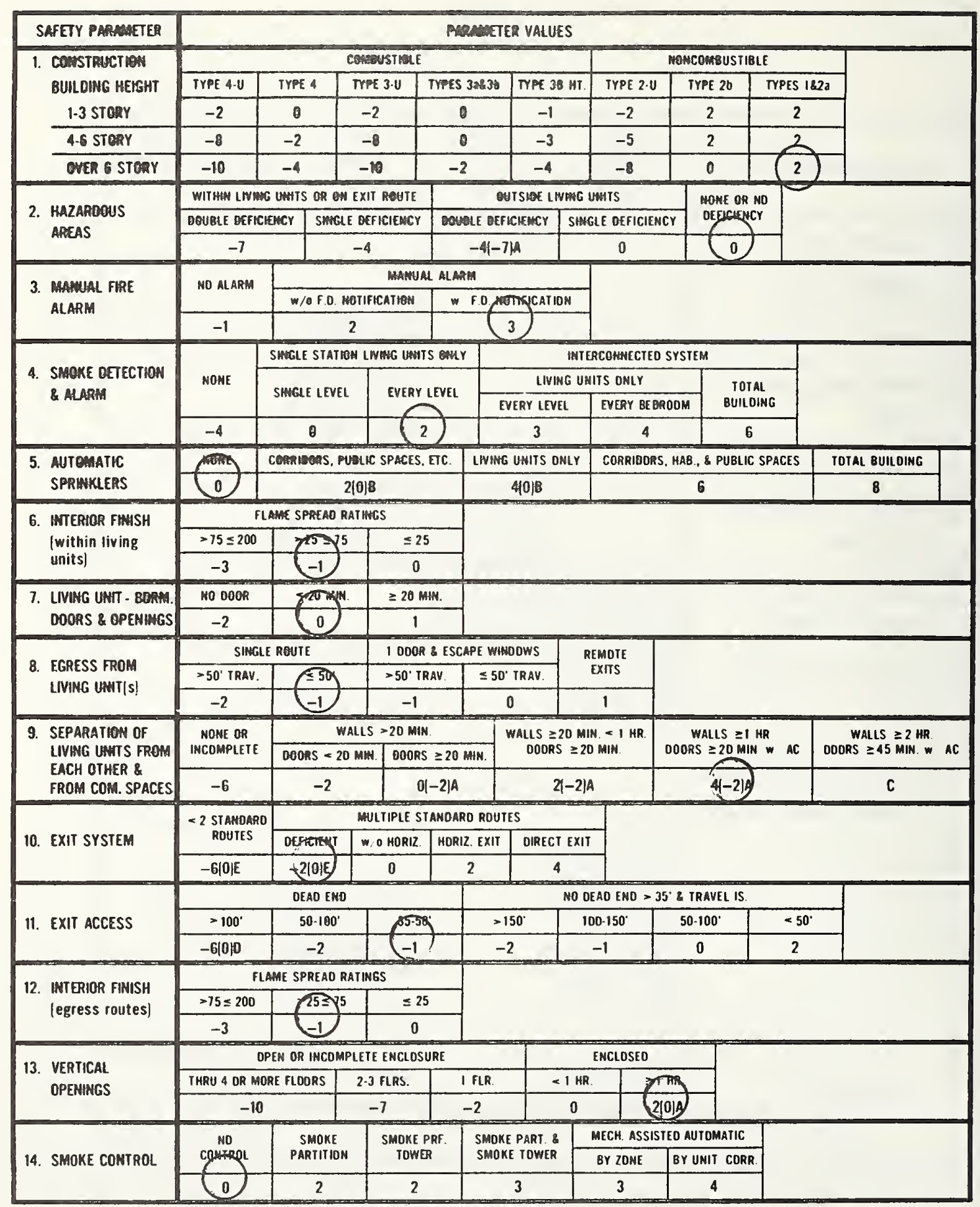

Figure 25a. MPS Requirements

Rehab. Housing for the Elderly

$>6$ story $-\leq 8$ units/floor 


\begin{tabular}{|c|c|c|c|c|c|}
\hline SAFETY PAMUNETER & 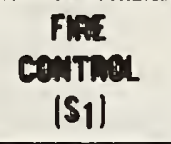 & $\begin{array}{l}\text { Eentss } \\
\text { mise }\end{array}$ & & $\begin{array}{l}\text { PEFUAE } \\
\text { ProvULED } \\
\text { [S3] }\end{array}$ & $\begin{array}{l}\text { GENERAL FIRE } \\
\text { SAFETY PROVIBED } \\
\text { (S4) }\end{array}$ \\
\hline 1. COASTRUCTIAM & 2 & & & 2 & 2 \\
\hline 2. HAZMEMUS ANEAS & 0 & $\div 2$ & 0 & 0 & 0 \\
\hline 3. MAMUAL FINE MLMAN & $\div 2 \quad 1.5$ & & 3 & & 3 \\
\hline $\begin{array}{l}\text { 4. SImOXE DETECTIOA \& } \\
\text { ALARin }\end{array}$ & $\div 2 \quad 1$ & & 2 & & 2 \\
\hline 5. AUTOMATIC SPRMAKLERS & 0 & $\div 2|0| B$ & 0 & {$[\div 2] A \quad 0$} & 0 \\
\hline 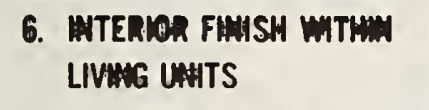 & $\div 2-.5$ & & & & -1 \\
\hline $\begin{array}{l}\text { 7. LIVHG LAIT - BORAM } \\
\text { DOORS \& OPENMES }\end{array}$ & & & & 0 & 0 \\
\hline $\begin{array}{l}\text { 8. EGRESS FROA LIYMG } \\
\text { UNIT/S| }\end{array}$ & & & -1 & & -1 \\
\hline $\begin{array}{l}\text { 9. SEPARATION OF LIMNG } \\
\text { UAITS FROM EACH OTHER } \\
\text { \& FROM COMHON SPACES }\end{array}$ & 4 & $\div 2$ & 2 & 4 & 4 \\
\hline 10. EXIT SYSTEM & & & -2 & -1 & -2 \\
\hline 11. EXIT ACCESS & & & -1 & & -1 \\
\hline $\begin{array}{l}\text { 12. WTERHOR FWWSH } \\
\text { (egress routes) }\end{array}$ & & & -1 & & -1 \\
\hline 13. VERTICAL OPEAWIGS & $\div 2 \quad 1$ & & 2 & 2 & 2 \\
\hline 14. SMOKE CONTROL & & & 0 & 0 & 0 \\
\hline TOTAL & $S_{1}=9$ & $S_{2}=$ & 4 & $S_{3}=7$ & $S_{4}=$ \\
\hline \multicolumn{6}{|c|}{$\begin{array}{l}\text { A - Use full value if Safety Parameter } 1 \text { is based on } 2-U, 3-U \text { or } 4-U \text { construction. } \\
\text { Divide by } 2(\div 2) \text { in all other cases. }\end{array}$} \\
\hline
\end{tabular}

Figure 25b. Mandatory Safety Requirements Rehab. Housing for the Elderly $>6$ story $-\leq 8$ units/floor 


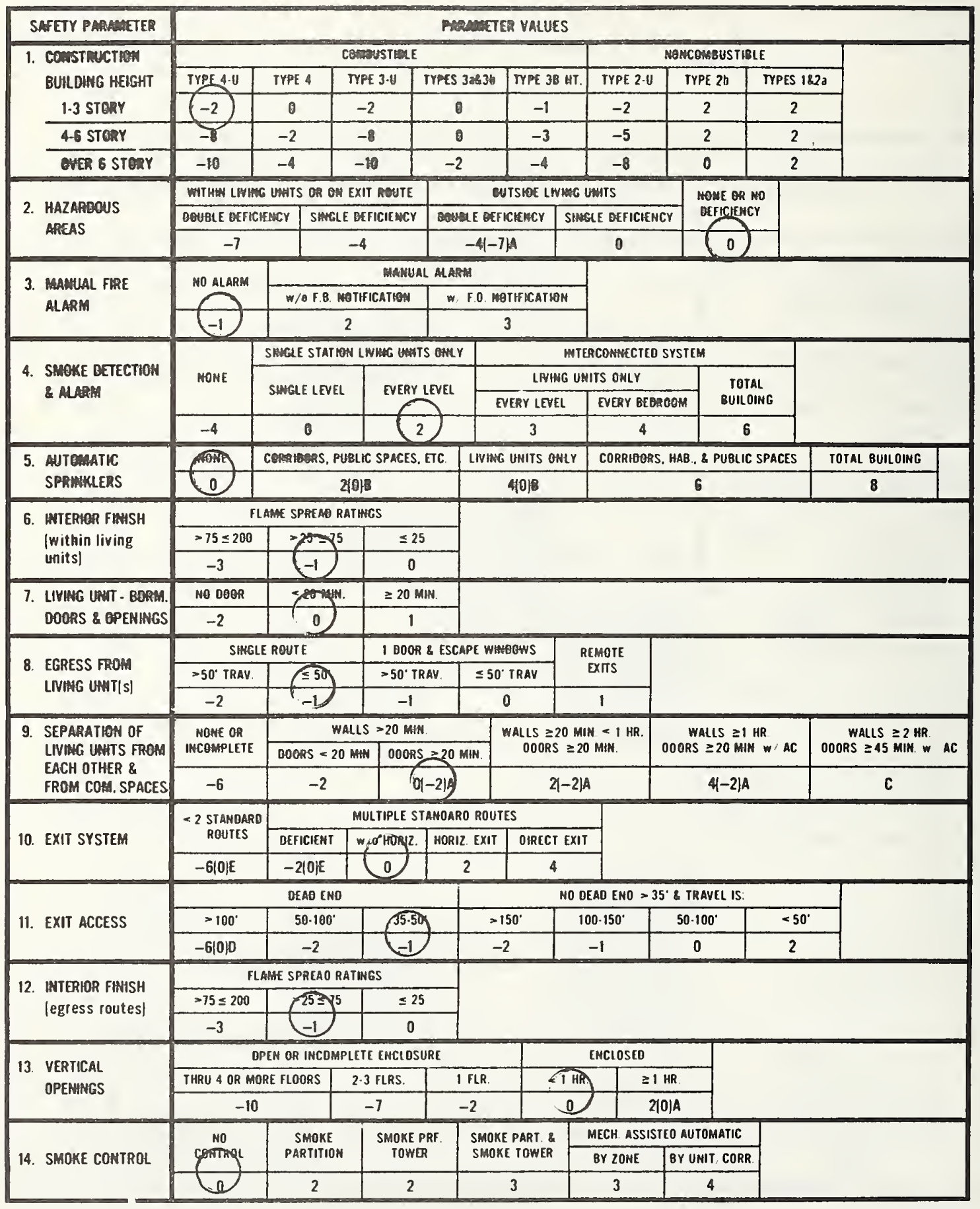

Figure 26a. MPS Requirements

Rehab. Housing for the Elderly

1-2 story - $\leq 8$ units/floor 


\begin{tabular}{|c|c|c|c|c|}
\hline SAFETY PAOMETER & 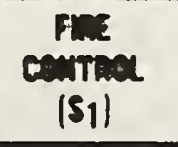 & 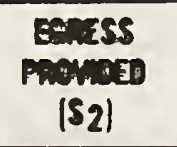 & $\begin{array}{l}\text { Drfyaz } \\
\text { PRavised } \\
\left(S_{3}\right)\end{array}$ & $\begin{array}{l}\text { GEMERUL FLEE } \\
\text { SAFETY PROMAED } \\
\text { (S4) }\end{array}$ \\
\hline 1. constractian & -2 & & -2 & -2 \\
\hline 2. Hazraans mishs & 0 & $\div 2$ & 0 & 0 \\
\hline 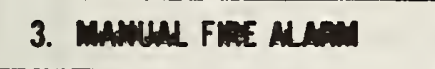 & $\div 2-.5$ & -1 & & -1 \\
\hline 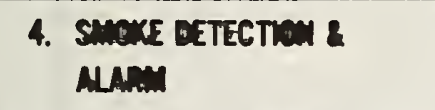 & $\div 2 \quad 1$ & 2 & & 2 \\
\hline 5. ANTCAMTIC SFAmikLERS & 0 & $\div 2 \mid$ 이: 0 & $(\div 2) A 0$ & 0 \\
\hline 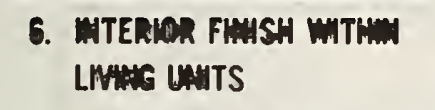 & $\div 2-.5$ & & & -1 \\
\hline $\begin{array}{l}\text { 7. LIVIMG UNAT - BQRM } \\
\text { DOORS \& OPEAMIS }\end{array}$ & & & 0 & 0 \\
\hline $\begin{array}{l}\text { 8. EGRESS FROW LIVMG } \\
\text { UHAT(S) }\end{array}$ & & -1 & & -1 \\
\hline $\begin{array}{l}\text { 9. SEPARATION OF LIVIMG } \\
\text { UWITS FROM EACH OTHER } \\
\text { \& FROM COMMON SPACES }\end{array}$ & -2 & $\div 2$ & -2 & -2 \\
\hline 10. EXIT SYSTEM & & 0 & $\div 2$ & 0 \\
\hline 11. EXIT ACCESS & & -1 & & -1 \\
\hline $\begin{array}{l}\text { 12. MTERTCR FWHSH } \\
\text { (egress routes) }\end{array}$ & & -1 & & -1 \\
\hline 13. VERTICML OPERMES & $\div 2 \quad 0$ & 0 & 0 & 0 \\
\hline 14. SHOKE CONTROL & & 0 & 0 & 0 \\
\hline TOTAL & $S_{1}=-4$ & $s_{2}=-3$ & $S_{3}=-4$ & $S_{4}=$ \\
\hline $\begin{array}{l}\text { NOTES: } \\
\text { A - Use full value if Safet } \\
\text { Divide by } 2(\div 2) \text { in all } \\
\text { B - Use }(0) \text { if operation of } \\
\text { alarm system. }\end{array}$ & $\begin{array}{l}\text { Parameter } 1 \mathrm{i} \\
\text { her cases. } \\
\text { rinkler syste }\end{array}$ & $\begin{array}{l}\text { based on 2-U } \\
\text { does not ac }\end{array}$ & $\begin{array}{l}3-U \text { or } 4-U \mathrm{co} \\
\text { ate building }\end{array}$ & $\begin{array}{l}\text { Istruction. } \\
\text { ire }\end{array}$ \\
\hline
\end{tabular}

Figure 26b. Mandatory Safety Requirements

Rehab. Housing for the Elderly

1-2 story - $\leq 8$ units/floor 


\begin{tabular}{|c|c|c|c|c|c|c|c|c|c|c|}
\hline SAFETY PARMOAETER & \multicolumn{9}{|c|}{ PRARTER VALUS } & \\
\hline \multirow{3}{*}{$\begin{array}{l}\text { 1. CENSTANCTIOA } \\
\text { BUHLHHG HEISHT } \\
\text { 1.3 STGRY }\end{array}$} & \multicolumn{6}{|c|}{ Convastiole } & \multicolumn{3}{|c|}{ MOMCOHEUSTRLE } & \\
\hline & TYPE 4.U & Jxas 4 & \multicolumn{2}{|c|}{ TYPE 3.U } & TrPES 3283a & THE 38 HT. & TYPE 2-U & TYPE 2b & TYPES 1820 & \\
\hline & -2 & $(0)$ & \multicolumn{2}{|c|}{-2} & $\theta$ & -1 & -2 & 2 & 2 & \\
\hline 4-6 STERY & -8 & -2 & \multicolumn{2}{|c|}{-8} & $\theta$ & -3 & -5 & 2 & 2 & \\
\hline OHER 6 STORY & -10 & -4 & -10 & & -2 & -4 & -8 & $\theta$ & 2 & \\
\hline & 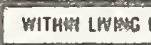 & HWTS OR O & EXIT & REUTE & & UTSLE LพIM & $G$ Uewts & Mane or $\mathrm{K}$ & & \\
\hline 2. HAZAREOUS & BQUELE BEFICIE & \begin{tabular}{l|l} 
encr & SMag \\
\end{tabular} & E EEF & Chency & DOAFE OEF & \begin{tabular}{l|l} 
ICrener & St
\end{tabular} & WGLE OEFICHEM & & & \\
\hline & -7 & & -4 & & $-41-7$ & & 0 & $(0)$ & & \\
\hline & NO ALARM & & & MANuNAL & AL Marim & & & & & \\
\hline 3. WAAUAL FRE & 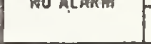 & $w / 0 F .0$ & of & ATE: & $w / f .0 . m$ & BTIFICATION & & & & \\
\hline & -1 & & 2) & & & 3 & & & & \\
\hline & & SHEQLE STATI & L & wo bers & is omir & & ITERCONHECTED & SYSTEM & & \\
\hline 4. SMENE OETECTION & HONE & 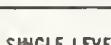 & & & & LWHA & UNITS OWLY & & & \\
\hline & & SWIGLE LEVE & & EVERY I & & VERY LEYEL & EVERY BEB & Bullo & & \\
\hline & -4 & 0 & & 2 & 2) & 3 & 4 & 6 & & \\
\hline 5. AUTOAAATK & 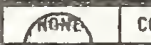 & COARIOenS, P & UBLIC & SPACES, & Livine & UNITS ONLY & CORRIQBRS & 5. HAB., \& PUBLIC & SPACES & TOTAL BUILDHG \\
\hline SPRWHKLERS & 0 & & 2 2아용 & & & $401 B$ & & 6 & & 8 \\
\hline 6. WTERLOR FAWSH & & AAC SPREAD & ATHAS: & & & & & & & \\
\hline (within living & $>75 \leq 200$ & $-25 \leq 7$ & & $\leq 25$ & & & & & & \\
\hline & -3 & $(-1)$ & & 0 & & & & & & \\
\hline 7. LIVHAG LAFT - BDRH. & NO 806 A & seons & & $\geq 20$ 베 & & & & & & \\
\hline DOORS \& OPENIMGS & -2 & 0 & & 1 & & & & & & \\
\hline & SINGLE & R.OUTE & & $1000 R$ & 28 ESCAPE WHA & 4Bews & REMOTE & & & \\
\hline 8. EGRESS FROH & $>50^{\circ}$ TRAY. & $=3 x$ & & $>50^{\prime} \mathrm{TR}$ & \begin{tabular}{l|l} 
RAY & $\leq 50$ \\
\end{tabular} & TRAY. & Exrs & & & \\
\hline & -2 & $(-1)$ & & -1 & & 0 & 1 & & & \\
\hline 9. SEPARATION OF & NONE OR & & ALLS & $-20 \mathrm{~min}$ & & WALLS $\geq 20$ & MIN $<1$ HR. & WALLS $\geq 1$ & & WALLS $\geq 2$ HR \\
\hline LINENG UNATS FROM & INCOMPLETE & DOens $<2$ & MAN. & BOORS & $S \geq 20$ min. & & $20 \mathrm{mIN}$ & $000 R S \geq 20$ MIN & 1. AC & OOORS $\geq 45$ MF. \\
\hline FROM COH. SPACES & -6 & -2 & & & $\{-2\} A$ & $21-2$ & & $(4-211)$ & & C \\
\hline & = 2 STAMOMRO & & mut & TIPLE ST & TANOARO ROUT & & & & & \\
\hline 10. EXIT SYSTEM & RQUTES & offrempis & & HORIZ. & HORIZ EXIT & OIRECT EX & & & & \\
\hline & $-6[0\} E$ & -21018 & & 0 & 2 & 4 & & & & \\
\hline & & DEAD ENI & & & & & OEAO ENO $>3$ & 5. TRAVEL IS: & & \\
\hline 11. EXIT ACCESS & $-100^{\circ}$ & $50.100^{\circ}$ & & 15.50 & & $150^{\circ}$ & \begin{tabular}{l|l}
$100 \cdot 150^{\circ}$ & \\
\end{tabular} & $50 \cdot 100^{\circ}$ & $<50^{\circ}$ & \\
\hline & $-6|0| 0 \mid 0$ & -2 & & -1 & -2 & & -1 & 0 & 2 & \\
\hline & & MEE SPREAO & ATING & & & & & & & \\
\hline $\begin{array}{l}\text { 12. IAIERIOR FWISA } \\
\text { |egress routes| }\end{array}$ & $\rightarrow 75 \leq 200$ & 253 & & $\leq 25$ & & & & & & \\
\hline & -3 & $(-1)$ & & 0 & & & & & & \\
\hline & & EN OR INCOM & PLETE & ENCLOSU & & & EHCLOSED & & & \\
\hline $\begin{array}{l}\text { 13. VERHCAL } \\
\text { OPENHES }\end{array}$ & THRU 4 OR MORE & RE FLOORS & 2.3 & FLRS. & $1 \mathrm{FLR}$. & -11 & \begin{tabular}{l|l} 
HR. & 71 \\
\end{tabular} & $\mathrm{HR}$ & & \\
\hline & -10 & & - & & -2 & 0 & & (01) & & \\
\hline & No & SMOKE & & SMOXE P & SHOKE & E PART. \& & MECH. ASSIST & TEO AUTOMATIC & & \\
\hline 14. SMOKE CONTROL & & PARTITIO & & TOWER & Shoox & KE TOWER & BY ZONE & BY UNIT CORR. & & \\
\hline & $(0)$ & 2 & & 2 & & 3 & 3 & 4 & & \\
\hline
\end{tabular}

Figure 27a. MPS Requirements

Rehab. Housing for the Elderly

3 story $-\leq 8$ units/floor 


\begin{tabular}{|c|c|c|c|c|}
\hline SAFETY PARAMETER & $\begin{array}{l}\text { FIRE } \\
\text { CONTROL } \\
\text { [S1] }\end{array}$ & $\begin{array}{l}\text { EGRESS } \\
\text { PROVIDED } \\
\text { (S2) }\end{array}$ & $\begin{array}{l}\text { REFUGE } \\
\text { PROVIDED } \\
\left(S_{3}\right)\end{array}$ & $\begin{array}{l}\text { GENERAL FIRE } \\
\text { SAFETY PROVIDED } \\
\left(S_{4}\right)\end{array}$ \\
\hline 1. CONSTRUCTION & 0 & & 0 & 0 \\
\hline 2. HAZARDOUS AREAS & 0 & $\div 2$ & 0 & 0 \\
\hline 3. MANUAL FIRE ALARM & $\div 2$ & 2 & & 2 \\
\hline $\begin{array}{l}\text { 4. SMOKE DETECTION \& } \\
\text { ALARM }\end{array}$ & $\div 2 \quad 1$ & 2 & & 2 \\
\hline 5. AUTOMATIC SPRINKLERS & 0 & $\div 2(0) \mathrm{B} \quad 0$ & $(\div 2) A 0$ & 0 \\
\hline $\begin{array}{l}\text { 6. INTERIOR FINISH WITHIN } \\
\text { LIVING UNITS }\end{array}$ & $\div 2-.5$ & & & -1 \\
\hline $\begin{array}{l}\text { 7. LIVING UNIT - BDRM } \\
\text { DOORS \& OPENINGS }\end{array}$ & & & 0 & 0 \\
\hline $\begin{array}{l}\text { 8. EGRESS FROM LIVING } \\
\text { UNIT/S| }\end{array}$ & & -1 & & -1 \\
\hline $\begin{array}{l}\text { 9. SEPARATION OF LIVING } \\
\text { UNITS FROM EACH OTHER } \\
\text { \& FROM COMMON SPACES }\end{array}$ & 4 & $\div 2$ & 4 & 4 \\
\hline 10. EXIT SYSTEM & & -2 & -1 & -2 \\
\hline 11. EXIT ACCESS & & -1 & & -1 \\
\hline $\begin{array}{l}\text { 12. INTERIOR FINISH } \\
\text { (egress routes) }\end{array}$ & & -1 & & -1 \\
\hline 13. VERTICAL OPENINGS & $\div 2$ & 2 & 2 & 2 \\
\hline 14. SMOKE CONTROL & & 0 & 0 & 0 \\
\hline TOTAL & $S_{1}=6.5$ & $S_{2}=$ & $S_{3}=$ & $S_{4}=$ \\
\hline \multicolumn{5}{|c|}{$\begin{array}{l}\text { NOTES: } \\
\text { A - Use full value if Safety Parameter } 1 \text { is based on } 2-U, 3-U \text { or } 4-U \text { construction. } \\
\text { Divide by } 2(\div 2) \text { in all other cases. } \\
\text { B - Use }(0) \text { if operation of sprinkler system does not activate building fire }\end{array}$} \\
\hline
\end{tabular}

Figure 27b. Mandatory Safety Requirements

Rehab. Housing for the Elderly

3 story $-\leq 8$ units/floor 


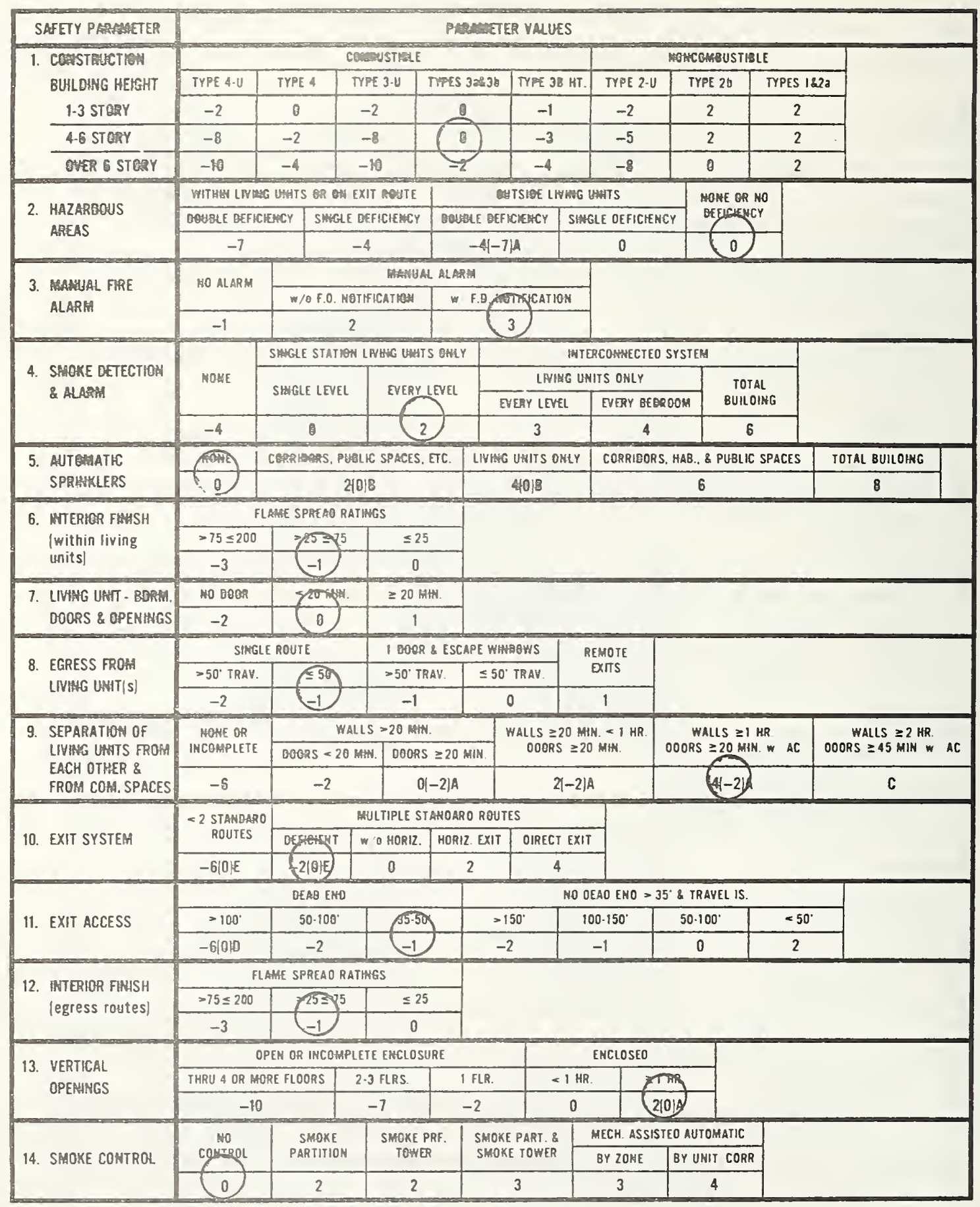

Figure 28a. MPS Requirements

Rehab. Housing for the Elderly

4-6 story $-\leq 8$ units/floor 


\begin{tabular}{|c|c|c|c|c|c|}
\hline SAFETY PARAMETER & $\begin{array}{l}\text { FIRE } \\
\text { CONTROL } \\
\text { (S1] }\end{array}$ & $\begin{array}{l}\text { EGRESS } \\
\text { PROVIDE } \\
{\left[S_{2}\right]}\end{array}$ & & $\begin{array}{l}\text { REFUGE } \\
\text { PROVIDED } \\
\text { (S3) }\end{array}$ & $\begin{array}{l}\text { GENERAL FIRE } \\
\text { SAFETY PROVIDED } \\
\text { [S4] }\end{array}$ \\
\hline 1. CONSTRUCTION & 0 & & & 0 & 0 \\
\hline 2. HAZARDOUS AREAS & 0 & $\div 2$ & 0 & 0 & 0 \\
\hline 3. MANUAL FIRE ALARM & $\div 2 \quad 1.5$ & & 3 & & 3 \\
\hline $\begin{array}{l}\text { 4. SMOKE DETECTION \& } \\
\text { ALARM }\end{array}$ & $\div 2 \quad 1$ & & 2 & & 2 \\
\hline 5. AUTOMATIC SPRINKLERS & 0 & $\div 2|0| B$ & 0 & $(\div 2) A 0$ & 0 \\
\hline $\begin{array}{l}\text { 6. INTERIOR FINISH WITHIN } \\
\text { LIVING UNITS }\end{array}$ & $\div 2-.5$ & & & & -1 \\
\hline $\begin{array}{l}\text { 7. LIVING UNIT - BDRM } \\
\text { DOORS \& OPENINGS }\end{array}$ & & & & 0 & 0 \\
\hline $\begin{array}{l}\text { 8. EGRESS FROM LIVING } \\
\text { UNIT(S) }\end{array}$ & & & -1 & & -1 \\
\hline $\begin{array}{l}\text { 9. SEPARATION OF LIVING } \\
\text { UNITS FROM EACH OTHER } \\
\text { \& FROM COMMON SPACES }\end{array}$ & 4 & $\div 2$ & 2 & 4 & 4 \\
\hline 10. EXIT SYSTEM & & & -2 & $\div 2$ & -2 \\
\hline 11. EXIT ACCESS & & & -1 & & -1 \\
\hline $\begin{array}{l}\text { 12. INTERIOR FINISH } \\
\text { (egress routes) }\end{array}$ & & & -1 & & -1 \\
\hline 13. VERTICAL OPENINGS & $\div 2 \quad 1$ & & 2 & 2 & 2 \\
\hline 14. SMOKE CONTROL & & & 0 & 0 & 0 \\
\hline TOTAL & $S_{1}=7$ & $S_{2}=$ & 4 & $s_{3}=$ & $S_{4}=$ \\
\hline \multicolumn{6}{|l|}{$\begin{array}{l}\text { NOTES: } \\
\text { A - Use full value if Safe } \\
\text { Divide by } 2(\div 2) \text { in all } \\
\text { B - Use }(0) \text { if operation of } \\
\text { alarm system. }\end{array}$} \\
\hline
\end{tabular}

Figure 28b. Mandatory Safety Requirements Rehab. Housing for the Elderly 4-6 story - $\leq 8$ units/floor 


\begin{tabular}{|c|c|c|c|c|c|c|c|c|c|c|c|}
\hline SAFETY PARAAETER & \multicolumn{10}{|c|}{ PARMETER YAUUES } & \\
\hline \multirow{3}{*}{$\begin{array}{l}\text { 1. COHSTRUCTHA } \\
\text { BULOHG HEISHT } \\
\text { 1-3 STORY }\end{array}$} & \multicolumn{7}{|c|}{ Comasstiale } & \multicolumn{3}{|c|}{ MOHCOMBUSTIGLE } & \\
\hline & IYPE 4-V & TYPE 4 & \multicolumn{2}{|c|}{ TYPE 3.4 } & \multicolumn{2}{|c|}{ TYPES $3 a \& 3 b$} & TYPE 38 НT. & TYPE $2 \cdot y$ & \multicolumn{2}{|r|}{ TYPES 162} & \\
\hline & -2 & 0 & \multicolumn{2}{|c|}{-2} & \multicolumn{2}{|c|}{0} & -1 & -2 & \multicolumn{2}{|r|}{2} & \\
\hline 4-6 STORY & -8 & -2 & \multicolumn{2}{|c|}{-8} & \multicolumn{2}{|c|}{$\theta$} & -3 & -5 & \multicolumn{2}{|l|}{2} & \\
\hline GNER 6 STORY & -10 & -4 & \multicolumn{2}{|c|}{-10} & -2 & & -4 & -8 & 0 & 2 & \\
\hline & WIT Hay LWMa & Uuts 0 & ExIT & คอแt & & & JTSBOE LNING & UWTS & & & \\
\hline 2. HAZARDOUS & DQUBAE BEFICIE & Eacy & LE DEF & ICIEECY & Bogr & ME BEF & \begin{tabular}{l|l} 
Rerency & SH \\
\end{tabular} & HLE DEFICIEN & & & \\
\hline & -7 & & -4 & & & $-4 \mid-7$ & & 0 & $(0)$ & & \\
\hline & HO & & & MaWUA & AL ALAF & & & & & & \\
\hline $\begin{array}{l}\text { 3. DAATUAL FERE } \\
\text { ALARA }\end{array}$ & & $w / 0$ & Markh & CATION & 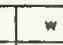 & $5.0 \%$ & FIEICAT HON & & & & \\
\hline & -1 & & 2 & & & & $\longrightarrow$ & & & & \\
\hline & & SMELE $S$ & IOW LN & IAG UMI & IS OAM & & & ERCD HAKECTED & SYSIEM & & \\
\hline 4. SWOKE DETECTION & NONE & & & & & & LAYIKG U & NITS ONLY & & & \\
\hline & & SANEL & & EVERY & NEL & & VERY LEYEL & EVERY BEBA & $200 \mathrm{M}$ & & \\
\hline & -4 & 0 & & 2 & $2 \longdiv { 2 }$ & & 3 & 4 & 6 & & \\
\hline 5. AUTOAATIC & HONE & conaren & ensic & SPACES. & ETC. & LIVAKG & UNTS ONIY & CORRIDOAS & S, HAB., \& PUBLIC & SPACES & TOTAL BUILOING \\
\hline SPRWXLERS & $\theta$ & & 24018 & & & & $40 / 8$ & & 6 & & 8 \\
\hline 6. HTERIQR FIMSH & & AME SPAE & RATHC & & & & & & & & \\
\hline Iwithin living & $>75 \leq 200$ & -25 & & $\leq 25$ & & & & & & & \\
\hline units] & -3 & & & 0 & & & & & & & \\
\hline 7. LIVNG UAT - BEAA & No 8009 & $<20$ & & $\geq 20 \mathrm{~m}$ & MIพ. & & & & & & \\
\hline DOORS \& OPENHGS & -2 & & & 1 & & & & & & & \\
\hline & SAMGLE & E ROUTE & & I DOOR & $R \&$ ESC & APE WIN & oows & REMOTE & & & \\
\hline 8. EGRESS FROM & $>50^{\circ}$ IRAV. & 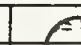 & & $>50^{\circ} \mathrm{TR}$ & RAV. & $\leq 50$ & TRAV. & ExITS & & & \\
\hline & -2 & & & -1 & & & 0 & 1 & & & \\
\hline 9. SEPARATION OF & NONE OR & & YALLS & $=20 \mathrm{MHN}$ & & & WALLS $\geq 20 \mathrm{~W}$ & $\mathrm{HN} \times 1 \mathrm{MR}$. & WALLS $\geq 1$ & HR. & WALLS $\geq 2$ HA. \\
\hline LIVING UATTS FROA & INCOMPLETE & ooons & 0 MแN. & 800RS & $S \geq 20$ & MiN. & $00085=0$ & 20 MIN. & $000 R S \geq 20$ Mir & & DOOAS $\geq 45$ MH. W \\
\hline FROM COM. SPACES & -6 & & & & $(-2) A$ & & $2\}-2$ & & $(1-213$ & & C \\
\hline & $<2$ SIANBARD & & & IIIPLE ST & TANOAF & 20 ROUT & & & & & \\
\hline 10. EXIT SYSTEM & ROUIES & 0689 & $w$ & O HORIZ. & HORI & 2. EXII & DIRECT EXI & & & & \\
\hline & $-6|0| E$ & -210 & & 0 & & 2 & 4 & & & & \\
\hline & & DEAD & & & & & No & OEAO ENO $>3$ & 35. 8 TRAVEL IS: & & \\
\hline 11. EXIT ACCESS & $>100^{\circ}$ & 50 . & & $55-50$ & & $>1$ & $50^{\circ}$ & $100-150^{\circ}$ & $50-100$ & $<50^{\circ}$ & \\
\hline & -61010 & - & & -1 & & - & & -1 & 0 & 2 & \\
\hline & & AME SPRE & RAJINE & & & & & & & & \\
\hline $\begin{array}{l}\text { 12. } \\
\text { (egress routes) }\end{array}$ & $-75 \leq 200$ & 370 & & $\leq 25$ & & & & & & & \\
\hline & -3 & - & & 0 & & & & & & & \\
\hline & & PEN OR IN & APLETE & ENCLOSL & SURE & & & ENCLOSEO & & & \\
\hline $\begin{array}{l}\text { 13. VERILAL } \\
\text { OPENANGS }\end{array}$ & THRU 4 OR $M O R$ & RE FLOOR & $2 \cdot 3$ & FLRS. & & FLR. & $<1 \mathrm{H}$ & & $\operatorname{Tin}$ & & \\
\hline & -10 & & & 7 & & -2 & 0 & & $(0) A$ & & \\
\hline & NO & & & SMOKE P & PRF. & smok & E PART. \& & MECH. ASSISI & SIED AUTOMATIC & & \\
\hline 14. SMOKE COHTROL & CONTROL & & & TOWE & & SMOK & E TOWER & BY ZONE & BY UNIT CORR & & \\
\hline & 0 & 2 & & 2 & & & 3 & 3 & $\overrightarrow{4}$ & & \\
\hline
\end{tabular}

Figure 29a. MPS Requirements

Rehab. Housing for the Elderly

$>6$ story - > 8 units/floor 


\begin{tabular}{|c|c|c|c|c|c|}
\hline SAFETY PARAMETER & $\begin{array}{l}\text { FIRE } \\
\text { CONTROL } \\
{\left[S_{1} \mid\right.}\end{array}$ & $\begin{array}{l}\text { EGRESS } \\
\text { PROVDEE } \\
\text { [S2] }\end{array}$ & & $\begin{array}{l}\text { REFUGE } \\
\text { PROVIDED } \\
\text { (S3) }\end{array}$ & $\begin{array}{l}\text { GENERAL FIRE } \\
\text { SAFETY PROVIDED } \\
\text { (S4) }\end{array}$ \\
\hline 1. CONSTRUCTION & 2 & & & 2 & 2 \\
\hline 2. HAZARDOUS AREAS & 0 & $\div 2$ & 0 & 0 & 0 \\
\hline 3. MANUAL FIRE ALARM & $\because 2 \quad 1.5$ & & 3 & & 3 \\
\hline $\begin{array}{l}\text { 4. SMOKE DETECTION \& } \\
\text { ALARM }\end{array}$ & $\div 2 \quad 1$ & & 2 & & 2 \\
\hline 5. AUTOMATIC SPRINKLERS & 2 & $\div 2(0) B$ & 1 & $(\div 2) A 1$ & 2 \\
\hline $\begin{array}{l}\text { 6. INTERIOR FINISH WITHIN } \\
\text { LIVING UNITS }\end{array}$ & $\div 2-.5$ & & & & -1 \\
\hline $\begin{array}{l}\text { 7. LIVING UNIT - BDRM } \\
\text { DOORS \& OPENINGS }\end{array}$ & & & & 0 & 0 \\
\hline $\begin{array}{l}\text { 8. EGRESS FROM LIVING } \\
\text { UNIT(S) }\end{array}$ & & & -1 & & -1 \\
\hline $\begin{array}{l}\text { 9. SEPARATION OF LIVING } \\
\text { UNITS FROM EACH OTHER } \\
\text { \& FROM COMMON SPACES }\end{array}$ & 4 & $\div 2$ & 2 & 4 & 4 \\
\hline 10. EXIT SYSTEM & & & -2 & $\div 2$ & -2 \\
\hline 11. EXIT ACCESS & & & -1 & & -1 \\
\hline $\begin{array}{l}\text { 12. INTERIOR FINISH } \\
\text { (egress routes) }\end{array}$ & & & -1 & & -1 \\
\hline 13. VERTICAL OPENINGS & $\div 2 \quad 1$ & & 2 & 2 & 2 \\
\hline 14. SMOKE CONTROL & & & 2 & 2 & 2 \\
\hline TOTAL & $S_{1}=11$ & $s_{2}=$ & 7 & $S_{3}=10$ & $s_{4}=$ \\
\hline \multicolumn{6}{|l|}{ Divide by $2(\div 2)$ in all } \\
\hline
\end{tabular}

Figure 29b. Mandatory Safety Requirements Rehab. Housing for the Elderly $>6$ story - > 8 units/floor 


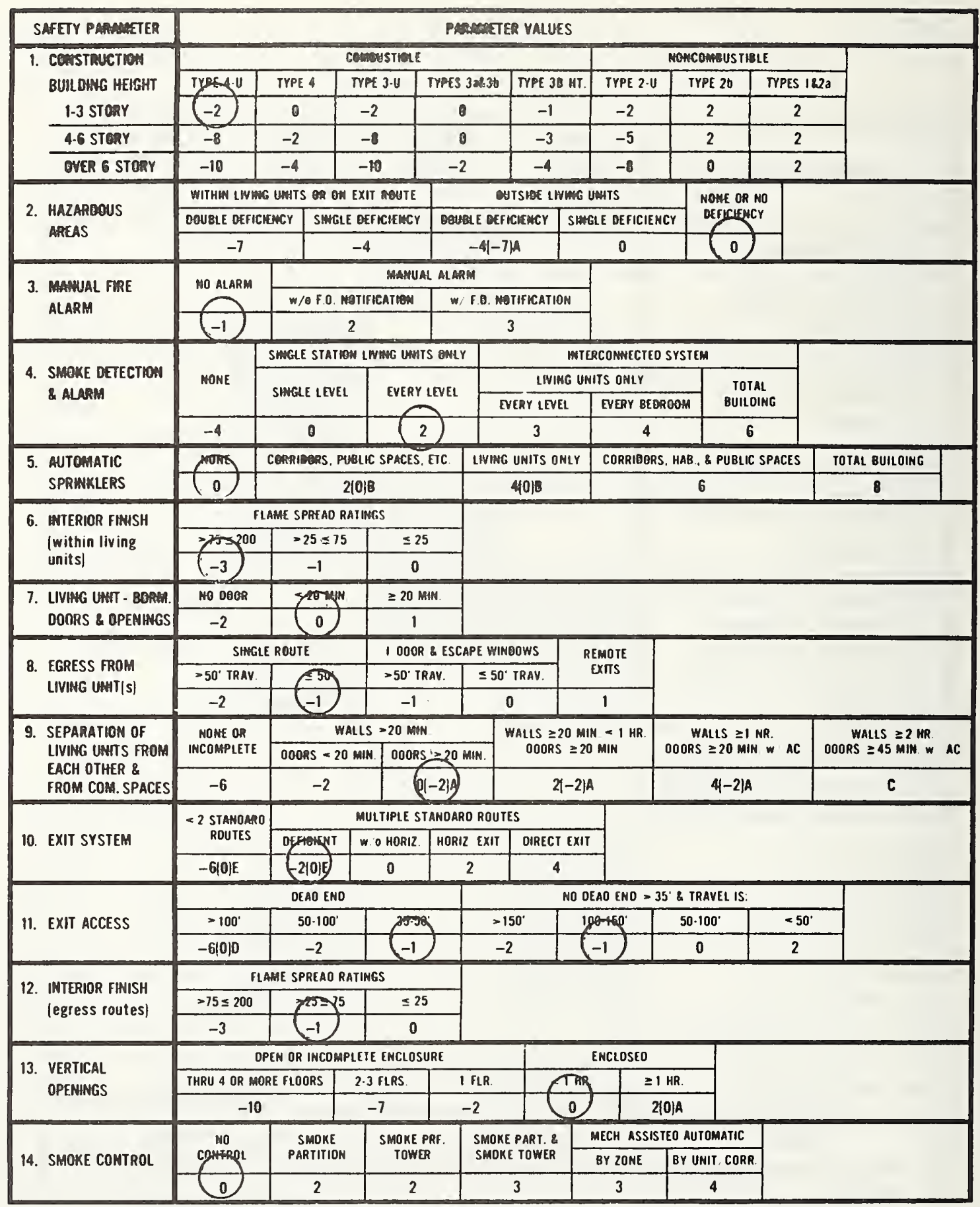

Figure 30a. MPS Requirements

Existing Multifamily and

Housing for the Elderly

1-2 story 


\begin{tabular}{|c|c|c|c|c|}
\hline SAFETY PARAMETER & $\begin{array}{l}\text { FIRE } \\
\text { COATROL } \\
{\left[S_{1} \mid\right.}\end{array}$ & $\begin{array}{l}\text { EGRESS } \\
\text { PROVDED } \\
\text { [S2] }\end{array}$ & $\begin{array}{l}\text { REFUGE } \\
\text { PROVIDED } \\
\text { (S } 3 \text { ) }\end{array}$ & $\begin{array}{l}\text { GENERAL FIRE } \\
\text { SAFETY PROVIDED } \\
\text { [S4] }\end{array}$ \\
\hline 1. CONSTRUCTION & -2 & $\sqrt{1}$ & -2 & -2 \\
\hline 2. HAZARDOUS AREAS & 0 & $\div 2 \quad 0$ & 0 & 0 \\
\hline 3. MANUAL FIRE ALARM & $\div 2-.5$ & -1 & & -1 \\
\hline $\begin{array}{l}\text { 4. SMOKE DETECTION \& } \\
\text { ALARM }\end{array}$ & $\div 2 \quad 1$ & 2 & & 2 \\
\hline 5. AUTOMATIC SPRINKLERS & 0 & $\div 2|0| B \quad 0$ & $(\div 2) \mathrm{A} 0$ & 0 \\
\hline $\begin{array}{l}\text { 6. INTERIOR FINISH WITHIN } \\
\text { LIVING UNITS }\end{array}$ & $\div 2-1.5$ & & & -3 \\
\hline $\begin{array}{l}\text { 7. LIVING UNIT - BDRM } \\
\text { DOORS \& OPENINGS }\end{array}$ & & & 0 & 0 \\
\hline $\begin{array}{l}\text { 8. EGRESS FROM LIVING } \\
\text { UNIT(S) }\end{array}$ & & -1 & & -1 \\
\hline $\begin{array}{l}\text { 9. SEPARATION OF LIVING } \\
\text { UNITS FROM EACH OTHER } \\
\text { \& FROM COMMON SPACES }\end{array}$ & -2 & -1 & -2 & -2 \\
\hline 10. EXIT SYSTEM & & -2 & $\div 2$ & -2 \\
\hline 11. EXIT ACCESS & & -1 & & -1 \\
\hline $\begin{array}{l}\text { 12. INTERIOR FINISH } \\
\text { (egress routes) }\end{array}$ & & -1 & & -1 \\
\hline 13. VERTICAL OPENINGS & $\div 2$ & 0 & 0 & 0 \\
\hline 14. SMOKE CONTROL & & 0 & 0 & 0 \\
\hline TOTAL & $S_{1}=-5$ & $S_{2}=-5$ & $S_{3}=$ & $S_{4}=$ \\
\hline \multicolumn{5}{|c|}{$\begin{array}{l}\text { NOTES: } \\
\text { A - Use full value if Safety Parameter } 1 \text { is based on } 2-U, 3-U \text { or } 4-U \text { construction. } \\
\text { Divide by } 2(\div 2) \text { in all other cases. } \\
\text { B - Use }(0) \text { if operation of sprinkler svstem does not activate building fire }\end{array}$} \\
\hline
\end{tabular}

Figure 30b. Mandatory Safety Requirements

Existing Multifamily and

Housing for the Elderly

1-2 story 


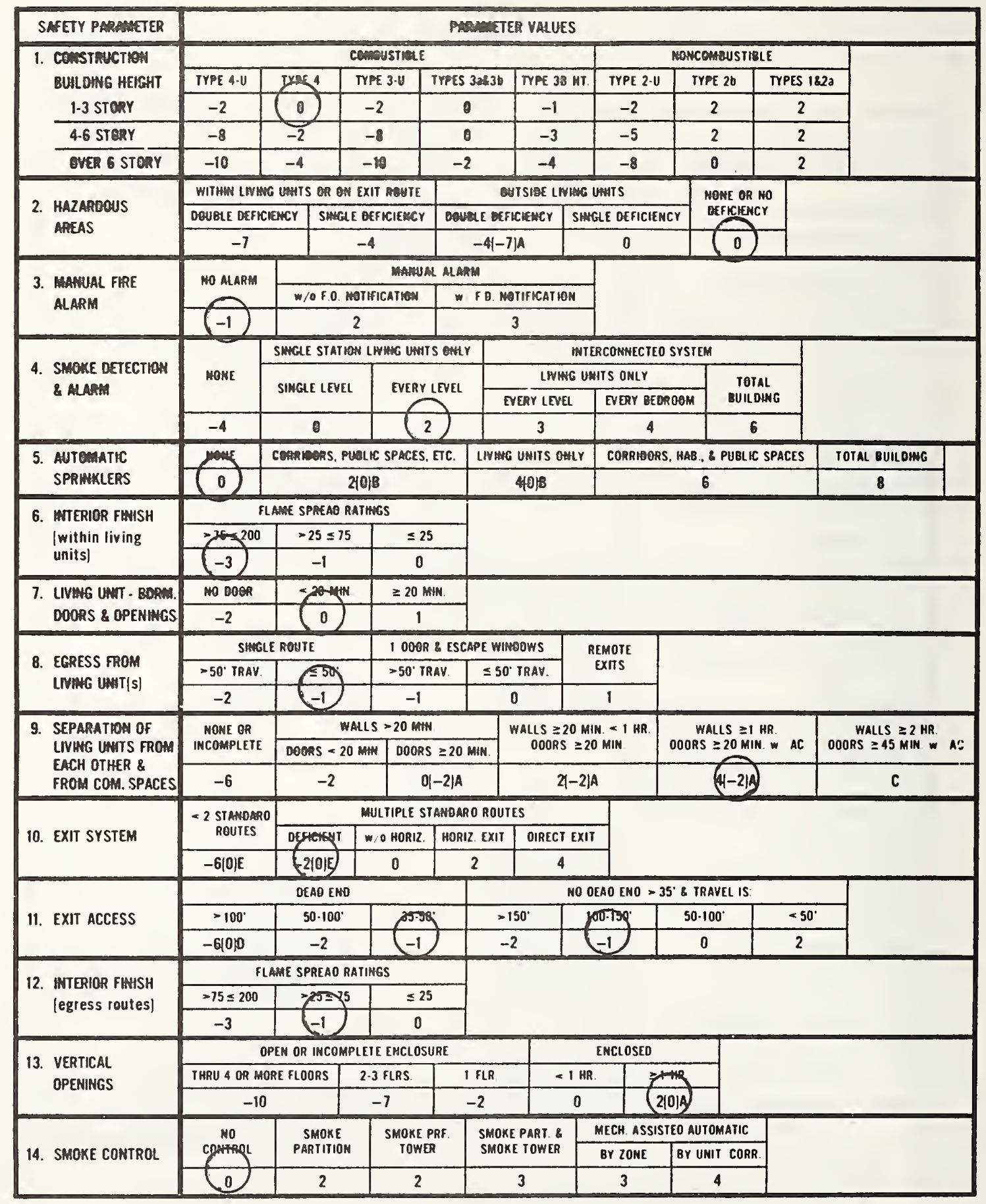

Figure 31a. MPS Requirements

Existing Multifamily and

Housing for the Elderly

3 story 


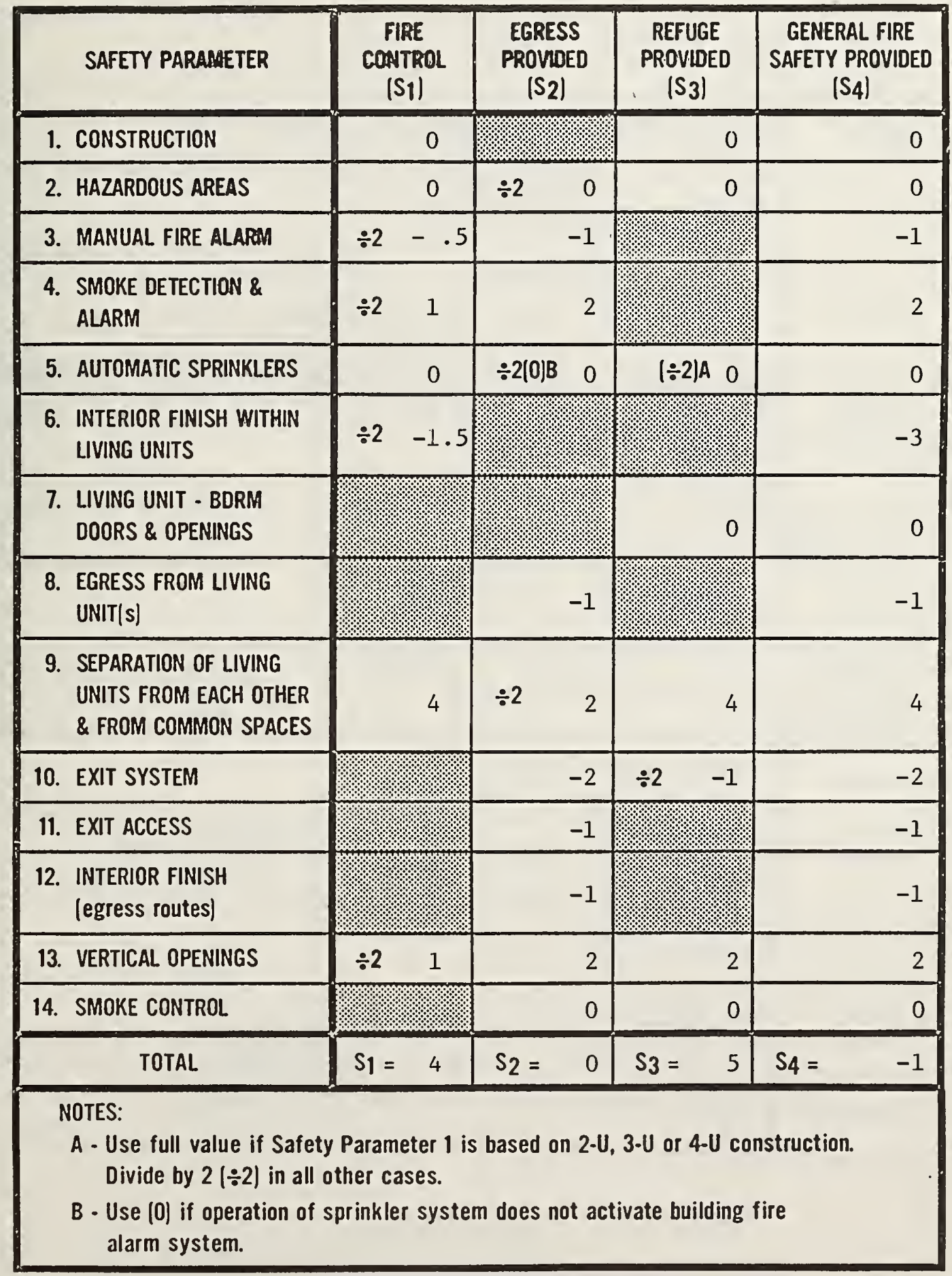

Figure 31b. Mandatory Safety Requirements

Existing Multifamily and

Housing for the Elderly

3 story 


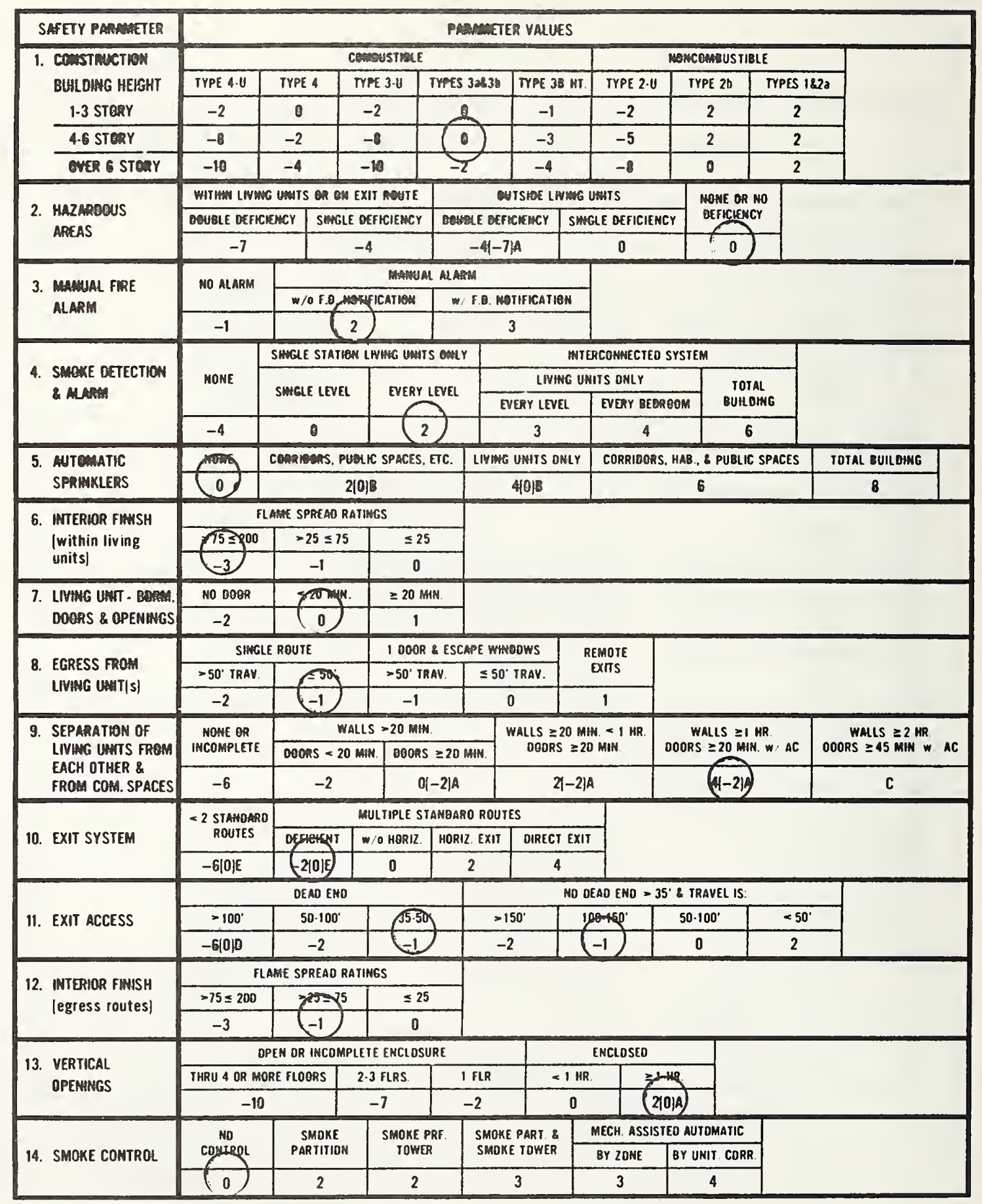

Figure 32a. MPS Requirements

Existing Multifamily and

Housing for the Elderly

4-6 story 


\begin{tabular}{|c|c|c|c|c|c|}
\hline SAFETY PARAMETER & $\begin{array}{l}\text { FIRE } \\
\text { COATROL } \\
\left|S_{1}\right|\end{array}$ & $\begin{array}{l}\text { EGRES } \\
\text { PROWD } \\
\text { [S2] }\end{array}$ & & $\begin{array}{l}\text { REFUGE } \\
\text { PROVIDED } \\
\left|S_{3}\right|\end{array}$ & $\begin{array}{l}\text { GENERAL FIRE } \\
\text { SAFETY PROVIDED } \\
\text { [S4] }\end{array}$ \\
\hline 1. CONSTRUCTION & 0 & & . & 0 & 0 \\
\hline 2. HAZARDOUS AREAS & 0 & $\div 2$ & 0 & 0 & 0 \\
\hline 3. MANUAL FIRE ALAPM & $\div 2$ & & 2 & & 2 \\
\hline $\begin{array}{l}\text { 4. SMMOKE DETECTION \& } \\
\text { ALARM }\end{array}$ & $\div 2 \quad 1$ & & 2 & & 2 \\
\hline 5. AUTOMATIC SPRIAKLERS & 0 & $\div 2(0) 8$ & 0 & $1 \div 2] \mathrm{A} \quad 0$ & 0 \\
\hline $\begin{array}{l}\text { 6. INTERIOR FINISH WITHIN } \\
\text { LIVING UNITS }\end{array}$ & $\div 2-1.5$ & & & & -3 \\
\hline $\begin{array}{l}\text { 7. LIVING UNIT - BDRM } \\
\text { DOORS \& OPENINGS }\end{array}$ & & & & 0 & 0 \\
\hline $\begin{array}{l}\text { 8. EGRESS FROM LIVING } \\
\text { UNIT(S) }\end{array}$ & & & -1 & & -1 \\
\hline $\begin{array}{l}\text { 9. SEPARATION OF LIVING } \\
\text { UNITS FROM EACH OTHER } \\
\text { \& FROM COMMON SPACES }\end{array}$ & 4 & $\div 2$ & 2 & 4 & 4 \\
\hline 10. EXIT SYSTEM & & & -2 & -1 & -2 \\
\hline 11. EXIT ACCESS & & & -1 & 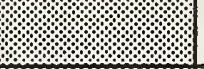 & -1 \\
\hline $\begin{array}{l}\text { 12. INTERIOR FINISH } \\
\text { (egress routes) }\end{array}$ & & & -1 & & -1 \\
\hline 13. VERTICAL OPENINGS & $\div 2$ & & 2 & 2 & 2 \\
\hline 14. SMOKE CONTROL & & & 0 & 0 & 0 \\
\hline TOTAL & $S_{1}=5.5$ & $s_{2}=$ & 3 & $s_{3}=$ & $S_{4}=$ \\
\hline \multicolumn{6}{|l|}{ Divide by $2(\div 2)$ in all } \\
\hline
\end{tabular}

Figure 32b. Mandatory Safety Requirements

Existing Multifamily and

Housing for the Elderly

4-6 story 


\begin{tabular}{|c|c|c|c|c|c|c|c|c|c|c|c|}
\hline SAFETY PARNAETER & \multicolumn{10}{|c|}{ PMANSIETER VALUES } & \\
\hline \multirow{3}{*}{$\begin{array}{l}\text { 1. CENSTAUETIOA } \\
\text { BUIDONG HEISHT } \\
1.3 \text { STERY }\end{array}$} & \multicolumn{7}{|c|}{ CemUUSTIOLE } & \multicolumn{3}{|c|}{ MOHCOMUUSTHELE } & \\
\hline & TYPE 4-U & TYPE 4 & \multicolumn{2}{|c|}{ TYPE 3.4 } & \multicolumn{2}{|c|}{ TYPES 3243b } & TYPE $3 B$ AT. & \multirow{2}{*}{$\frac{\operatorname{Irpt} 2 \cdot 0}{-2}$} & TYPE 2b & NYPES $182 a$ & \\
\hline & -2 & $\theta$ & \multicolumn{2}{|c|}{-2} & \multicolumn{2}{|c|}{ e } & -1 & & 2 & 2 & \\
\hline 4-6 STERY & -8 & -2 & \multicolumn{2}{|c|}{-8} & \multicolumn{2}{|c|}{$\theta$} & -3 & -5 & 2 & & \\
\hline GYER 6 STERY & -10 & -4 & $-k$ & & -2 & & -4 & -8 & 0 & 2 & \\
\hline & WITHAN LIVMG & 6 Uamis on & EXIT & ROUTE & & & TSIOE LWWO & Uwits & & & \\
\hline $\begin{array}{l}\text { 2. HALAFELUS } \\
\text { AREAS }\end{array}$ & DOUBLE DEFICK & ENCY & E BEF & reIENCY & 0040 & ME BEF & ICIEnCY & WGLE OEFICIEN & & & \\
\hline & -7 & & -4 & & & $-4 \mid-7$ & & 0 & 0 & & \\
\hline & MO & & & Manes & ALAR & & & & & & \\
\hline ALARM & 年 & $w / 0 F .0$ & 20451 & catsen & & Fo. Mo & JTFКСTION & & & & \\
\hline & -1 & & 2) & & & & 3 & & & & \\
\hline & & SWGLE STR & ON LN & ING UATT & $S$ OKLY & & & ILPCONHECTEO & SYSIEM & & \\
\hline 4. SHOKE DETECTION & NONE & surcicin & & & & & LNING U & UNITS ONLY & & & \\
\hline & & Sintole te & & EVent & & & VERY LEVEL & EVERY BED & BUILOH & & \\
\hline & -4 & 0 & & 2 & & & 3 & 4 & 6 & & \\
\hline 5. AUTOMATK & wing & CQRRteens & vetic & SPACES. & ETC. & LHพMั & UNITS ONLY & CORRIBOR & S. HAB., \& PUBLIC & SPACES & TOTAL BUILOHG \\
\hline SPRIAILLERS & 0 & & 2 이아B & & & & $4(0) B$ & & 6 & & 8 \\
\hline 6. MTERIOR FWUSH & & AME SPREA & RATHO & & & & & & & & \\
\hline Iwithin living & $75 \geq 9^{00}$ & $-25 \leq$ & & $\leq 25$ & & & & & & & \\
\hline units) & -3 & -1 & & 0 & & & & & & & \\
\hline 7. LIWHG UWT - BRAM. & NO OOOR & $=20 x$ & & $\geq 20 \mathrm{~m}$ & & & & & & & \\
\hline DOORS \& OPENHGS & -2 & 0 & & 1 & & & & & & & \\
\hline & SIAGLE & E ROUTE & & $1000 R$ & 2 ESCA & APE WAN & oows & REMOTE & & & \\
\hline 8. EGRESS FOM & >50' IRAY. & $\leq 5$ & & $\Rightarrow 50^{\prime} \mathrm{Th}$ & RAV. & $\leq 50^{\circ}$ & TRAV. & ExTs & & & \\
\hline 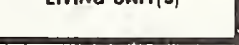 & -2 & $(-1$ & & -1 & & s & 0 & 1 & & & \\
\hline 9. SEPARATION OF & NONE OR & & IALLS & $>20$ MAN & & & WALLS $\geq 20 \mathrm{~m}$ & MIN. \& 1 HR. & WALLS $\geq 1 \mathrm{H}$ & & WALLS $\geq 2$ HR. \\
\hline LWWG UATS FROM & INCOMPLETE & DocRs - & 0 min. & OOORS & $\geq 201$ & & DOORS $\geq 2$ & 20 MIN & OOORS $\geq 20 \mathrm{M}$ & & DOORS $\geq 45$ MIN. $w$ \\
\hline FROM COM. SPACES & -6 & -2 & & & $(-2) A$ & & $21-2$ & & $(4-2)$ & & C \\
\hline & $<2$ stanoneo & & MUL & TIPLE SI & TANOAR & O ROUT & & & & & \\
\hline 10. EXIT SYSTEM & ROUTES & Descerty & $w / 0$ & O HORIZ. & HORI2 & EXIT & OIRECI EXI & & & & \\
\hline & $-6|0| E$ & -2||ㅣ & & 0 & & 2 & 4 & & & & \\
\hline & & OEAOE & & & & & & OEAO ENO > 3 & $15^{\circ}$ \& TRAVEL IS: & & \\
\hline 11. EXIT ACCESS & $>100^{\circ}$ & $50 \cdot 10$ & & $25 \times 56$ & & -1 & $50^{\circ}$ & $100-150^{\circ}$ & $50 \cdot 100^{\circ}$ & $<50^{\circ}$ & \\
\hline & $-610 \mid 0$ & -2 & & -1 & & -2 & & -1 & 0 & 2 & \\
\hline & & AWE SPREA & TATHE & & & & & & & & \\
\hline (egress routes) & $>75 \leq 200$ & $205=$ & & $\leq 25$ & & & & & & & \\
\hline & -3 & $(-1$ & & 0 & & & & & & & \\
\hline & & PEN OR INC & PLETE & ENCLOS & URE & & & ENCLOSEO & & & \\
\hline OPENANGS & THRU 4 OR MOR & RE FLOORS & $2 \cdot 3$ & FLRS. & & FLR. & $<1 \mathrm{H}$ & & & & \\
\hline & -10 & & - & 7 & & -2 & 0 & & $10 \mid 2$ & & \\
\hline & NO & SMOK & & SMOKE F & & SMOKE & E PART. 8 & MECH. ASSIS & TEO AUTOMATIC & & \\
\hline 14. SHOKE CONTROL & CONIRgL & PARTIT & & TOWE & & SMOK & E TOWER & BY ZONE & BY UNIT CORR. & & \\
\hline & 0 & 2 & & 2 & & & 3 & 3 & 4 & & \\
\hline
\end{tabular}

Figure 33a. MPS Requirements

Existing Multifamily and

Housing for the Elderly

$>6$ story 


\begin{tabular}{|c|c|c|c|c|}
\hline SAFETY PARAMAETER & $\begin{array}{l}\text { FIRE } \\
\text { CONTROL } \\
{\left[S_{1} \mid\right.}\end{array}$ & $\begin{array}{l}\text { EGRESS } \\
\text { PROVDED } \\
\text { (S2) }\end{array}$ & $\begin{array}{l}\text { REFUGE } \\
\text { PROVIDED } \\
\text { (S3) }\end{array}$ & $\begin{array}{l}\text { GENERAL FIRE } \\
\text { SAFETY PROVIDED } \\
\left(S_{4}\right)\end{array}$ \\
\hline 1. CONSTRUCTION & 2 & & 2 & 2 \\
\hline 2. HAZARDOUS AREAS & 0 & $\div 2$ & 0 & 0 \\
\hline 3. MANUAL FIRE ALARAA & $\div 2$ & 2 & & 2 \\
\hline $\begin{array}{l}\text { 4. SMOKE DETECTION \& } \\
\text { ÁLARM }\end{array}$ & $\div 2 \quad 1$ & 2 & & 2 \\
\hline 5. AUTOMATIC SPRINKLERS & 0 & $\div 2|0| \mathrm{B} \quad 0$ & $(\div 2) \mathrm{A} 0$ & 0 \\
\hline $\begin{array}{l}\text { 6. INTERIOR. FINISH WITHIN } \\
\text { LIVING UNITS }\end{array}$ & $\div 2-1.5$ & & & -3 \\
\hline $\begin{array}{l}\text { 7. LIVING UNIT - BDRM } \\
\text { DOORS \& OPENINGS }\end{array}$ & & ঝ. & 0 & 0 \\
\hline $\begin{array}{l}\text { 8. EGRESS FROM LIVING } \\
\text { UNIT(S) }\end{array}$ & & -1 & & -1 \\
\hline $\begin{array}{l}\text { 9. SEPARATION OF LIVING } \\
\text { UNITS FROM EACH OTHER } \\
\text { \& FROM COMMON SPACES }\end{array}$ & 4 & $\div 2$ & 4 & 4 \\
\hline 10. EXIT SYSTEM & & -2 & -1 & -2 \\
\hline 11. EXIT ACCESS & & -1 & & -1 \\
\hline $\begin{array}{l}\text { 12. INTERIOR FINISH } \\
\text { (egress routes) }\end{array}$ & & -1 & & -1 \\
\hline 13. VERTICAL OPENINGS & $\div 2$ & 2 & 2 & 2 \\
\hline 14. SMOKE CONTROL & & 0 & 0 & 0 \\
\hline TOTAL & $S_{1}=7.5$ & $S_{2}=3$ & $s_{3}=$ & $S_{4}=$ \\
\hline \multicolumn{5}{|c|}{$\begin{array}{l}\text { NOTES: } \\
\text { A - Use full value if Safety Parameter } 1 \text { is based on } 2-U, 3-U \text { or } 4-U \text { construction. } \\
\text { Divide by } 2(\div 2) \text { in all other cases. } \\
\text { B - Use (0) if operation of sprinkler system does not activate building fire }\end{array}$} \\
\hline
\end{tabular}

Figure 33b. Mandatory Safety Requirements Existing Multifamily and Housing for the Elderly $>6$ story 
1. Nelson, H. E., and Shibe, A. J., "A System for Fire Safety Evaluation of Health Care Facilities", NBSIR 78-1555-1, National Bureau of Standards, Washington, D. C., May, 1980.

2. National Fire Protection Association, "Life Safety Code, 1981. Appendix C", NFPA, Boston, 1981.

3. Department of Housing and Urban Development, Revision No. 9 "Minimum Porperty Standards for Multifamily Housing 4910.1", HUD, Washington; D. C., March, 1980.

4. National Fire Protection Association, "Fire Safety Concepts Tree", NFPA, Boston, 1980.

5. Watts, J.; "The Goal Oriented Systems Approach," NBS-GCR-77-103, National Bureau of Standards, Washington, D. C. , July, 1977.

6. Dalley, N. and Helmer, D., "An Experimental Application of the Delphi Method to the Use of Experts," Management of Science 9, No. 3 p. 458, April, 1963. 
APPENDIX A

NBS DELPHI GROUP

\section{Delphi Method}

The Delphi technique was developed in the 1950's for the purpose of estimating the probable effects of atomic bombing attacks on the United States. Since then it has been applied to technological forecasting as well as in areas where judgmental information is required. The Delphi technique is basically concerned with the utilization of the combined knowledge of experts to arrive at a consensus opinion where factual information is incomplete.

The NBS exercise followed a process called Policy Delphi. The basic premise of the Policy Delphi is that it acts as a precursor to a committee activity. The Policy Delphi is not a substitute for research studies, analyses, or staff work. It is, however, an organized method for correlating views and information pertaining to a specific problem area and for allowing the respondents representing such views and information the opportunity to react to and assess differing viewpoints. Because the respondents are anonymous, fear of potential repercussions or embarrassment is removed and no simple individual need commit himself publicly to a particular view until after the alternatives have been put on the table.

Turoff in "The Policy Delphi"* analyzed committee and Delphi processes. The study points out that a Delphi followed by a committee provides good results in formulating policies.

The study identifies two major areas of problems with large size committees (i.e., communication and psychological). The communication difficulties are attributed to the diverse membership. The major lack of understanding tends to be between the following groups: individuals who are not familiar with many of the new decision aids coming out of operation research and system analyses but who have an intuitive feel for the complexities of the organization, and individuals who have been trained in many of modern management techniques and who are sometimes a little too confident that these approaches can be applied to every problem. The problems associated with operation of committees that tend to reflect psychological characteristics are:

- The domineering personality, or outspoken individual that takes over committee process.

- The unwillingness of individuals to take a position on an issue before all facts are in or before it is known which way the majority is headed.

*Murray Turoff, "The Design of a Policy Delphi", Technological Forecasting and Social Changes 2, No. 2 (1970). 
- The difficulty of publicly contradicting individuals in higher positions.

- The unwillingness to abandon a position once it is publicly taken.

- The fear of bringing up uncertain ideas that may turn out to be idiotic and results in loss of face.

The above limitation may also apply to small size committees, except when the members of the small committee are given sufficient time to consider and explore the issue, and have assurance that the privacy of their respective remarks will be respected outside the committees. Under those conditions a small committee will not have any of the difficulties which have been identified for the large size committee.

Usually Delphi, whether it is to be conventional or computerized, undergoes four distinct phases. The first phase is characterized by exploration of the subject under discussion, wherein each individual contributes additional information he feels is pertinent to the issue. The second phase involves the process of reaching an understanding of how the group views the issue. If there is significant disagreement among members, the disagreement is explored in the third phase to bring out the underlying reasons for differences and possibly to evaluate them. The last phase, a final evaluation, occurs when all previously gathered information has been initially analyzed and the evaluations have been fed back for consideration.

There are two methods of gaining consensus: conventional and computerized. In the conventional form, a monitor team designs a questionnaire which is sent to a respondent group. After the questionnaire is returned, the monitor team summarizes the results, and based upon the results develops a revised questionnaire for the respondent group to answer. The respondent group is usually given at least one opportunity to revise its original answers after examing the group response.

The computerized method replaces the monitor group to a large degree with a computer which has been programmed to carry out the compilation of the respondent group results. This process has the advantage of eliminating delays in summarizing each round of Delphi, thereby turning the process into a real-time communication system. However, it does require that the information received from the respondents is in a form that can be fed into a computer and that an algorithm can be provided to analyze the data. The NBS Delphi Group used the conventional four-phase approach in its evaluation process. 
Approach Used in Developing Fire Safety Parameters and Their Values

\section{CFR Delphi Group}

Fourteen individuals from the Fire Safety Engineering Division of the Center for Fire Research were chosen to act as a "Delphi" group. The experience of the group members in areas of fire/life safety ranged from six to thirty-five years. (Table A-1 identifies the group members.) Each individual was briefed about the general nature of the life safety risk analysis system and was given a detailed description of the safety model. The individuals were encouraged to seek more information about the system or any individual parameter, if the information given to them was insufficient. No guidance was provided as to the importance of any redundancy system or individual parameter.

\section{Instructions for Completing of Forms}

Each member of the Delphi Group was given five separate but identical forms, one for each of five fire safety functions: 1) General Fire Safety; 2) Fire Development; 3) Fire Containment; 4) Emergency Egress; and 5) Emergency Refuge. Delphi members were told the safety requirements should be considered only as they apply to multifamily housing. They were also instructed that the safety impact of each safety parameter should be established on its importance to a total facility safety basis. Each form had a clear statement of the specific safety function it should evaluate. General instructions for filing the questionnaire were:

1) Evaluate the relative worth of the safety requirement, (i.e. parameter) on each of the five fire safety functions, one function at a time, and record conclusions on the appropriate questionnaires.

2) Use numerical values to express the level of safety for each subdivision of each parameter.

3) The range of numerical values should not exceed $(+10)$ for the highest level of safety of $(-10)$ for the condition presenting the most severely hazardous condition. It is not necessary to use both $(+10)$ and $(-10)$. Such should occur only if the safety value of the most important safeguard exactly compensates the risk imposed by the most detrimental element. If this is not true the maximum safety value and maximum risk number should not be identical. Where the parameter's status neither improves safety nor creates a hazardous condition, a "zero" value should be assigned.

4) Add additional safety parameters to any of the questionnaires, if required to provide a more complete safety evaluation.

5) Increase the number of parameter subdivisions, if the number shown on the forms is insufficient.

6) Remarks may be made on each of the five forms. 
Analysis of the Questionnaires

1. Forms. The completed forms were checked for completeness, illegible numbers and remarks. Where required, individuals were asked to provide additional information to complete the questionnaire. Figure A-2 shows the format agreed to by the Delphi group.

2. Preparation of Parameter Values. Each individual was requested to submit 350 values, which made the process of adjustments quite laborious. The values for each safety level were clustered to identify where major deviations occurred. An individual who supplied values significantly different from the cluster was asked for the reasoning behind his choice. Mostly the differences were generated by misinterpretations of the safety parameter functions. The values were then adjusted by the individuals and the process of preparing a consensus safety parameter table began.

3. Safety Parameter Table. Fourteen safety parameters were chosen to represent the most important areas of fire safety in multifamily buildings. The individual safety parameter values were adjusted using arithmetic means. All the values were expressed as whole numbers rounded off toward the "conservative" side.

4. Safety Parameter Selection for the Redundancy Systems. It is generally recognized that not all safety parameters are of equal importance in providing safety for a particular redundancy fire safety system. To identify those parameters which provide significant safety levels for each of the proposed redundancy systems the following method was used. For each redundancy system a set of three tables was generated. The first table had all the values of each parameter as assigned by the individual Delphi member. The second table was similar to the first, except numerical values were clustered in six ranks. The ranks are: High (10-8); Medium (7-4); Low (3-0); Negative Low (-1 to -3); Negative Medium $(-4$ to -7$)$; and Negative High $(-8$ to -10$)$. The third table ranked the safety parameters according to whether they provided high safety values or small safety values. Parameters with high safety values were included in the particular redundancy equation. The low value parameters were excluded from the equations because their ability to affect the total safety of a particular redundancy system was marginal. A number of safety parameters could not be evaluated by this system. The Delphi members could not agree on a general value for those safety parameters.

About one half of the members assigned high safety values to those parameters, where the other half assigned low safety values for the same parameters. Additional Delphi group query did not change their initial parameter values. To reflect the Delphi group split, the safety values of those parameters were divided by one-half. 
The distribution of values assigned by Delphi members to two proposed redundancy systems, Fire Development and Fire Containment, were almost identical. Therefore, the Fire Development and the Fire Containment systems were combined to form a single redundancy system, Fire Control.

Fourteen safety parameters were included into the Fire Safety Evaluation System as providing significant life/fire safety support. of those, four were assigned to all three redundancy systems (Hazardous Areas; Automatic Sprinklers; Separation of Living Units; and Vertical Openings). Five parameters were assigned to two redundancy systems (Fire Alarm; Smoke Detection; Exit System; Smoke Control; and Construction). The other fire safety parameters appear only once in the redundancy systems.

5. Delphi Group Status. The Delphi group finished its prime assignment to provide the basic system to be analyzed by the outside consultant group. The Delphi group also met several times after finishing this initial assignment, to consider adjustments or changes to the system suggested by the outside consultants or identified through NBS research. At each meeting the group analyzed the problem and suggested possible improvements to the system. 
Table A-1

NBS Delphi Group

Benjamin, Irwin

Division Chief, Structural Engineer

Bright, Richard (Retired)

Sr. Fire Protection Engineer

Budnick, Edward

Program Head, Fire Protection Engineer

Bukowski, Richard

Acting Division Chief, Electrical Engineer

Cooper, Leonard

Fire Prevention Engineer

Custer, Richard (Retired)

Division Chief, Fire Protection Engineer

Gomberg, Al

Acting Program Head, Fire Protection Engineer

Gross, Daniel

Senior Mechanical Engineer

Lee, Bill

Fire Protection Engineer

Nelson, Harold

Program Head, Fire Protection Engineer

o'Neill, John

Fire Protection Engineer

Parker, William

Physicist

Peacock, Richard

Chemical Engineer

Vogel, Bertrum (Retired)

Structural Engineer 


\section{APPENDIX B \\ PEER GROUP}

The Peer Group consisted of prominent persons in the regulation or specification of fire safety for multifamily housing. This included regulatory officials, code writing officials, fire safety consultants and architects representing a cross section of the applied field. The group membership is shown in Table $B-1$. The dates of meetings and attendance are shown in Table B-2.

\begin{tabular}{|c|c|c|}
\hline Name & Affiliation & Representation \\
\hline Austin, $W$. & $\begin{array}{l}\text { HUD, Public Housing } \\
\text { Architect }\end{array}$ & Regulatory \\
\hline Belles, D. & $\begin{array}{l}\text { Independent Consultant } \\
\text { Fire Protection Engineer }\end{array}$ & NFPA Life Safety Code \\
\hline Burgun, A. & Roger, Butler \& Burgun & NFPA Life Safety Code \\
\hline Prassas, M. & Independent Architect & $\begin{array}{l}\text { American Institute of } \\
\text { Architects (AIA) }\end{array}$ \\
\hline Gangnes, A. & Independent Architect & Public \\
\hline Gray, G. & Independent Architect & Pub1ic \\
\hline Lynch, R. & Independent Consultant & Public \\
\hline Moore, D. & HUD, Research Center & Regulatory \\
\hline Roux, H. & Armstrong Cork Company & NFPA Life Safety Code \\
\hline Sanders, J. & $\begin{array}{l}\text { Fire Marshal } \\
\text { State of Oklahoma }\end{array}$ & Code Enforcing \\
\hline Summers, H. & $\begin{array}{l}\text { Fire Marshal } \\
\text { State of Virginia }\end{array}$ & Code Enforcing \\
\hline Morehart, J. & $\begin{array}{l}\text { Department HHS, Ofc. of Sec. } \\
\text { Fire Safety Engineer }\end{array}$ & Regulatory \\
\hline
\end{tabular}


Table B-2

DATES OF MEETINGS AND ATTENDANCE

Meeting

January 31 - February 1, 1980 Washington, D.C.

\section{Attendance}

Belles, D.

Gangnes, A.

Gray, G.

Roux, $\mathrm{H}$.

Moore, D.
Burgun, A.

Lynch, R.

Morehart, J.

Sanders, J.

\section{Meeting}

June 11-12, 1980

Washington, D.C.

\section{Attendance}

Belles, D.

Burgun, A.

Gangnes, A.

Morehart, J.

Gray, G.

Lynch, R.

Roux, H.

Prassas, M.

Moore, D.

Sanders, J.

\section{Meeting}

September 8-9, 1981

Washington, D.C.

\section{Attendance}

Gangnes, A.

Gray, G.

Roux, H.

Moore, D.

Burgun, A.
Morehart, J. Lynch, $R$.

Prassas, M. Austin, W. 
Meeting

Apri1 14-15, 1981

Washington, D.C.

\section{Attendance}

Belles, D.

Gangnes, A.

Gray, G.

Burgun, A.

Prassas, M.
Austin, W.

Summers, H.

Morehart, J.

Moore, D.

Sanders, J. 


\section{Section A - Program Description}

This computer program was developed to support the proof testing of the evaluation system. It is designed to be used in determining how best to upgrade a multi-family residence to an acceptable level of fire safety. The user inputs one or more values for each of the 14 safety parameters, each value being either the value of the corresponding parameter in the current state of the facility, or a "higher" value that would result from an upgrading of the facility. The program examines all possible combinations of input values of the 14 parameters, one value per parameter, and prints up to 1000 combinations that would upgrade the safety of the facility to or above the mandatory safety requirements if implemented.

If a given configuration of values for the 14 safety parameters yields a facility the safety of which is above the mandatory fire safety requirements, then any configuration in which some of the safety parameters are given "higher" values and in which no parameter values are decreased will also satisfy the requirements. For this reason, in any set of configurations the minimal configurations of parameter values that satisfy the mandatory safety requirements will be called "basic" solutions, and these are the only ones that the computer program will print. In the remainder of this discussion unless otherwise specified all solutions mentioned will be basic without explicitly calling attention to this fact.

The solutions, or successful combinations, may be sorted by one chosen parameter if the user wishes, in which case up to 1000 solutions may be printed for each value of the sorting parameter, with the precise number being user-determined. The special sorting feature provides the capability of analyzing building configurations which are limited to certain characteristics.

As presently written, the program searches for up to 1111 solutions for each specified value of the sorting routine, limiting the output for each value to at most the first 1000 solutions. A single change in input eliminates the sorting feature, causing all solutions to be grouped together and allowing a change in the total printed output to any number less than 1000 solutions. In all cases, a limit on the available combinations, or building configurations, may be accomplished by limiting the possible combinations of the parameters on input. The program is written in non-standard Fortran, and may not run on a particular machine unless minor modifications are made. 


\section{Subsection B-1 - Card Setup and Description}

There are 19 cards in each set of data, and any number of sets may be run at one time. For sample input streams the reader should refer to Section F.

$\begin{array}{cl}\text { Card No. } & \text { Description } \\ 1 & \text { Title - To appear at top } \\ & \text { of each page of output. } \\ & \text { Number of values for the } \\ & \text { parameter Construction, } \\ & \text { followed by the values. } \\ & \text { After the last one appears } \\ & \text { the number of the specified } \\ & \text { Construction values which } \\ & \text { correspond to unprotected } \\ & \text { situations. }\end{array}$

3
Number of values for Hazardous Areas, followed by the values.

Manual Fire Alarm

Smoke Detection and Alarm

Automatic Sprinklers

Interior Finish (within Living Units)

Living Unit - Bedroom

Egress from Living Unit(s)

Separation of Living Units from Each Other and From Common Spaces

Exit System

Exit Access

Interior Finish (Egress

Routes)

Vertical Openings

Smoke Control
Doors and Openings

\section{Format of Data on Card}

In columns $1-72$.

Any characters may be used.

Data may begin in any column, must be integer, and must be separated by blanks. A minus sign is used for negative numbers. No commas or decimal points are allowed. No character may appear in column 80. Any number of blanks can be used to separate the values and ascending order of the values is required.

Same as for card 2.

See card 2.

See card 2.

See card 2.

See card 2.

See card 2.

See card 2.

See card 2.

See card 2.

See card 2.

See card 2.

See card 2.

See card 2. 
Card No.

16

17

18

19
Minimum total Safety

See card 2.

Values - Enter af ter multiplying by 10 the values from Table 3, depending on type of building and characteristics being examined. When the combinations are being examined by the program, those with any Table 2 sums less than the corresponding minimum total safety values will not be solutions.

Maximum Total Safety Values These serve to delimit the number of solutions. Each value must be greater than or equal to the corresponding minimum total safety value. When choosing a solution, the program will select only those combinations all of whose Table 2 sums are greater than or equal to their corresponding minimum total safety values, but such that not all the sums are greater than their corresponding maximum total safety values.

Number of Printed Solutions Desired - Will be the total number of solutions printed if sorting feature not used, or will be the number of solutions printed per each value of the sorting parameter if the sort feature is used. If greater than 1000 it will be changed to 1000 .

Number of the Sorting Parameter - 1-14 if the See card 2. sort routine is desired, 0 if not. If the sort is used solutions will be grouped into clusters. Each member of a cluster will have the same number for the sorting parameter, this value being higher than the one the sort parameter had in the last cluster. Sometimes the sort parameter is called the parameter under analysis.
See card 2.

See card 2. 
'l'he program output has two main formats. One format is generated when the user does not wish to use the sort feature, with the solutions appearing in one group. The other is used when the sort feature is specified, and the solutions appear grouped by ascending values of the sort parameter. The two formats and their differences will be illustrated by the output Examples 1 and 2 in Section G. The unsorted format will now be discussed, then the ways in which the sorted output differs from it.

The reader should now examine the output of Example 1. The first output page shows all of the input with the exception of the number of printed solutions desired. At the top of the page appears the desired title, followed by the parameters which are given only one input value and the values of each. If there are no parameters that are held constant the word "none" appears under the heading. Following are the parameters which have more than one input value and the values which are used. Construction is treated specially, with a "u" written after values which correspond to unprotected situations. Below the safety parameter values on the page are written the specified minimum and maximum total safety values.

The printed solutions appear next. The number on the extreme left under the heading "SOL非" is the solution number corresponding to the successful combination on the right. One may observe that the columns under the heading "cases" are blank for all 32 solutions, indicating that no solution printed satisfied a special case. The special cases and corresponding flags will be discussed in the next section on sorting routine output since there are many special situations shown in the second example. To the right of the solution number and cases columns appear the values of the safety parameters in the successful combinations, under headings which are abbreviated forms of the parameter names. Parameters which are held constant do not appear here, since their values are the same for each solution, and the reader is reminded that these constant values are an integral part of the combinations, especially in computing the four total safety sums. These four sums appear to the right of the nonconstant parameter values, and are computed from the combinations using Table 2 after its entries are multiplied by 10 .

The solutions are printed until either all solutions have been exhausted or the specified print limit has been reached, with the title appearing at the top of each page. At the end of the output a message is written in one of three forms:

XXXXX COMBINATIONS HAVE BEEN FOUND.

or XXXXX COMBINATIONS HAVE BEEN FOUND. FIRST XXXXX HAVE BEEN PRINTED.

or MORE THAN 1111 COMBINATIONS HAVE BEEN FOUND. FIRST XXXXX HAVE BEEN PRINTED.

These messages appear at the top of the page following the one on which the last output appeared. When the number of solutions is less than or equal to the number of printed solutions desired, the first message is written. Otherwise not all solutions are printed, and one of the other two messages appears. 
The reader should now refer to example 2, in which the sorting routine is used, and parameter number 7 (Living Unit Bedroom) is chosen as the sort parameter. The first page of output is identical to example 1 except that a message flagging the sorting parameter and its.values has been added at the bottom.

If one examines the entire output, taking account of the headings at the top of each page, one notices how the solutions are grouped by ascending values of the sorting parameter. Each group has a format almost identical to the total solution output of the unsorted case, with one exception, the heading between the title and the column headings. Also it may be seen that for the combinations using the first value of the sort parameter the special heading begins with the word "combinations" while for the combinations in the other groups it begins with "new combinations". This is because solutions which are successful with a certain value of the sort parameter will always be successful with a higher value of the parameter, and hence in such a situation the later "duplicate" solution is suppressed. Another fact to note is that the parameter being examined does not appear in the listing of solution parameter values; in effect it is treated as a constant parameter within each grouping.

In example 2 it can be seen that many of the solutions are flagged to the right of the solution number under the "cases" heading. These flags, the letters $A-D$, of which only $A$ and $B$ appear here, indicate the special cases that can occur. They are listed at the bottom of Table 1 .

If a solution fulfilled special case $A$ it would be flagged by an $A$ in the column under the $C$ in "Cases". If it satisfied case B it would have a B under the A in "Cases", and so on, the flag for each case being incremented in both value and column. The reason the flags need to be in distinct columns may be graphically observed in solutions 5 through 7 , of example 2, which fulfill more than one special case simultaneously. In those solutions which are flagged it is usually possible to discern one or more parameter values to the right of which an asterisk appears. This is a flag indicating that the flagged parameter value is adjusted because of the special case. It does not always appear because the parameter the value of which is modified may be a constant parameter or the sort parameter, in which case its value is not printed.

Finally, if in the non-sort case no solutions were found, or if in the sortspecified case no solutions were located for a particular value of the sort parameter, a message appears indicating that there are no combinations which satisfy the conditions.

The equivalency testing program is supplied with internal comments, and therefore the programmer should have at his disposal a copy of the source listing. Information on the inputs and outputs of the program is contained in this report. Together, these two sources supply almost all the guidance any programmer would need to understand and, if necessary, modify the existing code. This programmer's guide is therefore very general and does not dwell on each minute point of the program.

Section $D$ consists of a flowchart. Section $E$ is a listing of the main variables used and their functions. A listing of the program is included in Section F, while Section $G$ consists of the input card images for Examples 1 and 2 , followed by the example outputs. 


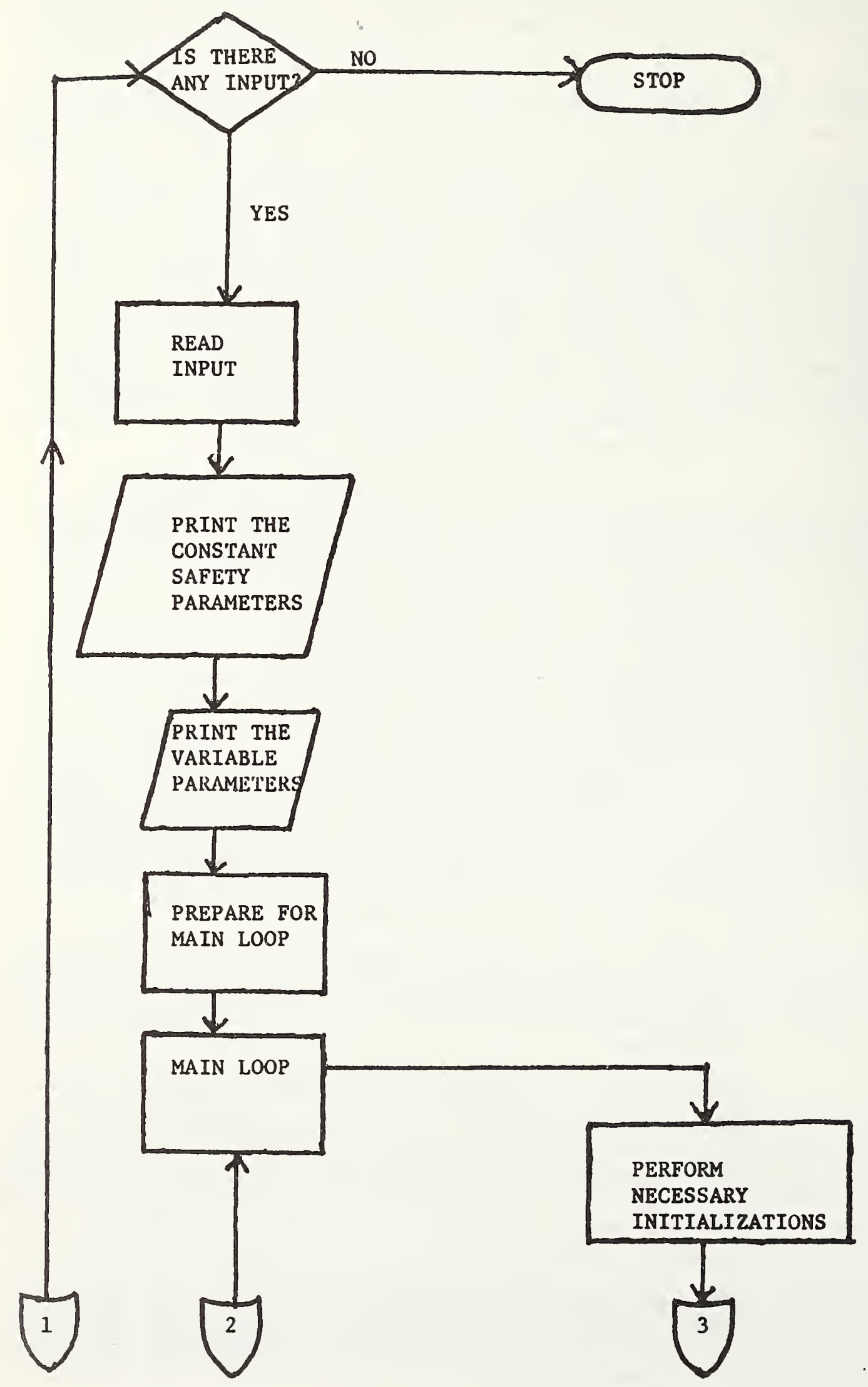




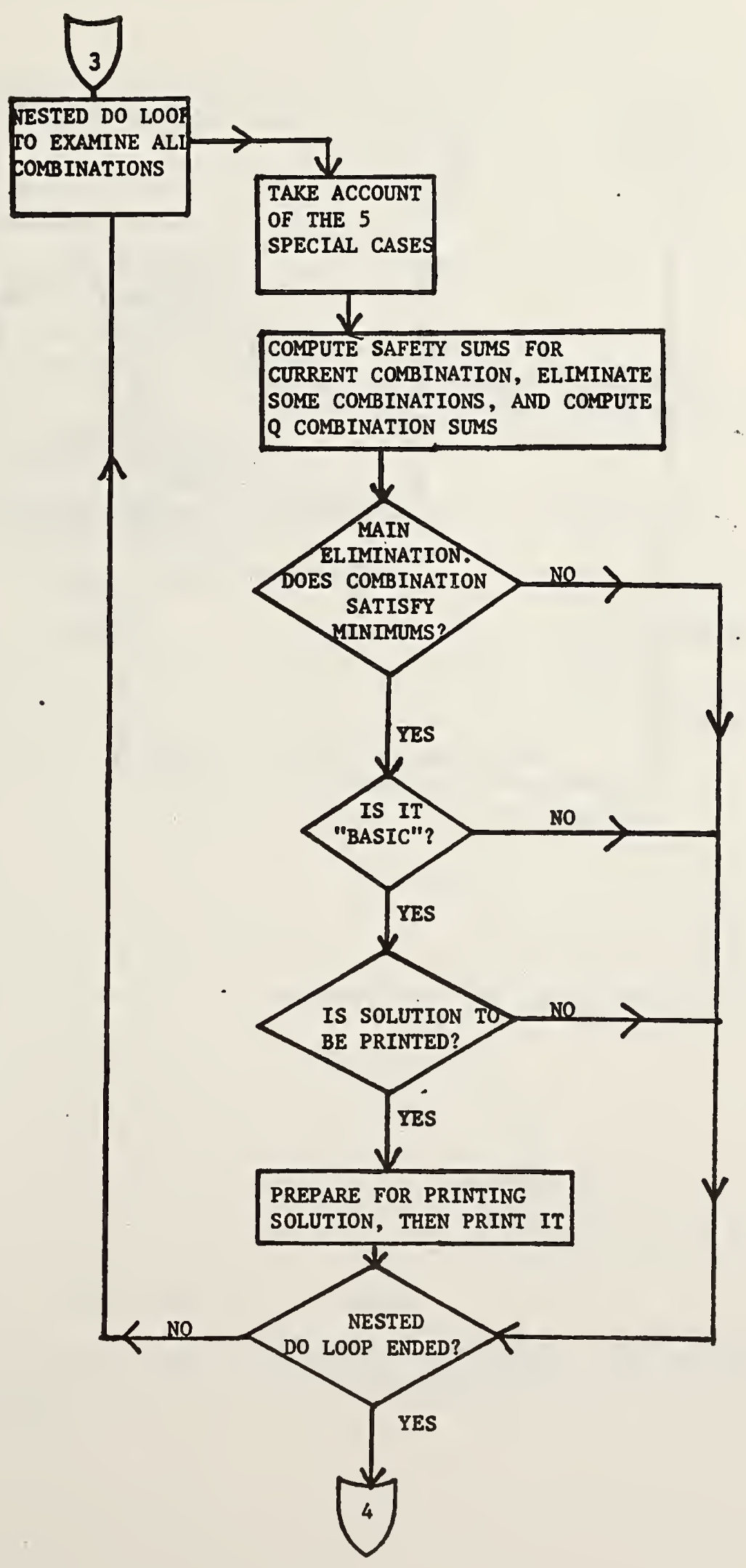




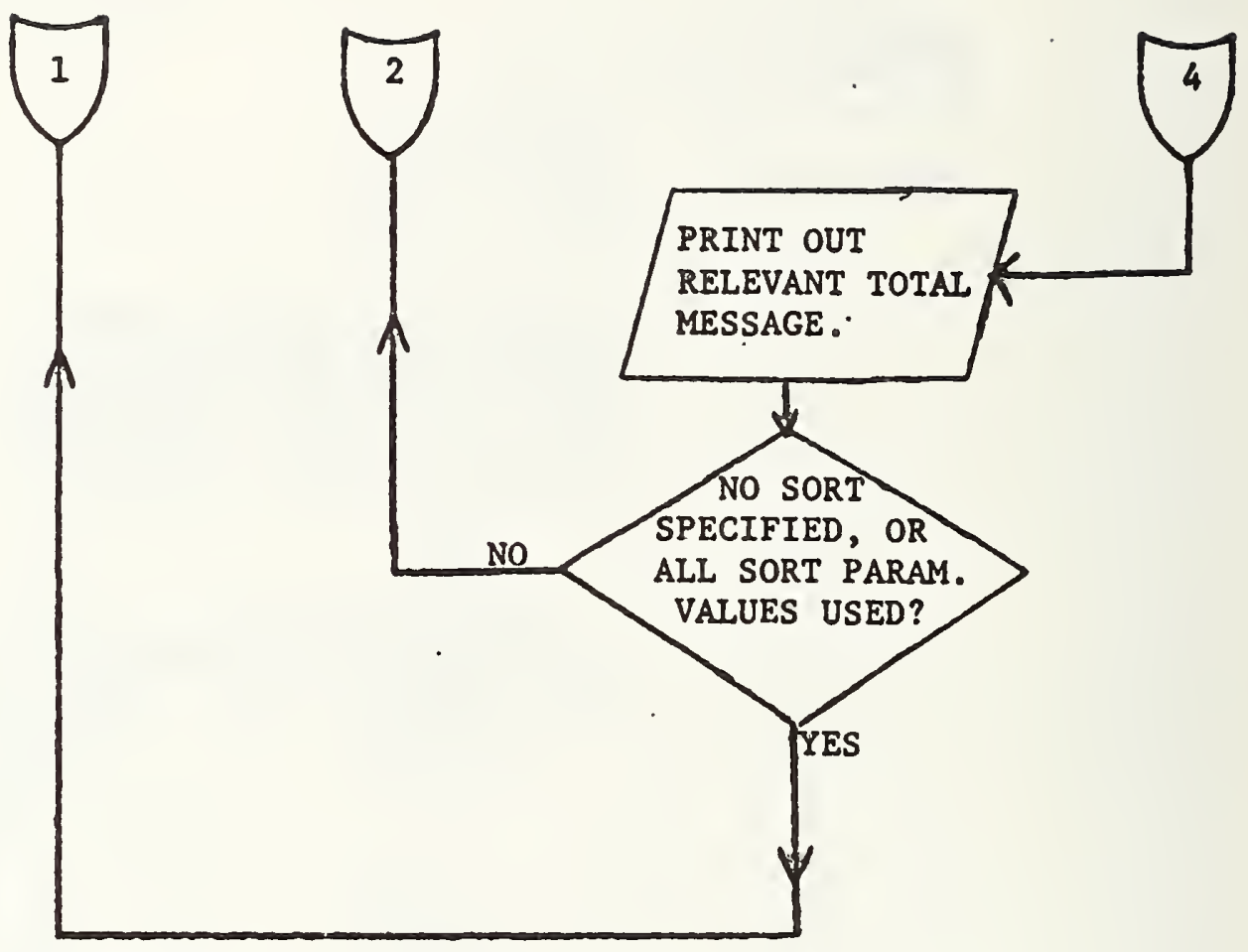


Section E - Main Variables of the Program

1. ALTER - An indicator that the last combination to be checked satisfied a special case.

2. BLANK - A variable containing a blank which is used for various purposes.

3. BLC - The number of values the sort parameter is given on input.

4. BLN - The sort parameter number.

5. CHANGE - An array of pointers to the nonconstant parameters. If CHANGE $(1)=5$, the fifth parameter is the first nonconstant one, to give an example.

6. CONDS - Print flags indicating the special case numbers 1-5.

7. CT - Number of nonconstant parameters.

8. CUR - The current values in main loop of the 14 safety parameters, initialized as $\operatorname{cur}(1)=\mathrm{P}(1, \mathrm{I} 1), \operatorname{cur}(2)=\mathrm{P}(2, \mathrm{I} 2), .$.

9. CYCLES - Number of solutions found in all if no sort is specified, or for the current sort parameter value.

10. FLAG - Used in most situations where a flag is required.

11. I - I(I) is equivalent to I1, I(2) is equivalent to I2, etc.

12. ICOUNT - Number of solutions printed for each solution grouping.

13. I1-I14 - Used to reference parameters $1-14$ in the arrays $P$ and $Q$.

14. $J-J(1)$ is equivalent to $J 1, J(2)$ to $J 2$, etc.

15. J1-J14 - The number of values of parameters 1-14.

16. KT - Number of the current execution of the main loop.

17. LAST - The value of the sort parameter during the last loop pass.

18. LINES - Lines written so far on a page.

19. LINESP - The number of the solution to be printed. For example, if LINESP $=500$, the solution to be output will be the 500 th printed. 
20. MMAX - Desired number of printed solutions for each grouping. Must be less than or equal to 1000 .

21. NA - Variable changed from $\emptyset$ to 1 if some of conditions in note $A$ which cause a safety parameter value to be changed are satisfied for last value of the sort parameter.

22. NA2 (NA9) - Variable changed for $\emptyset$ to 1 if conditions in note A causing modification of safety parameter 2 (9) are satisfied for last value of sort parameter.

23. NEWT - Parameter to record test result for current values of safety parameters to see whether or not configuration is dominated by a solution previously obtained.

24. NTA (NTB, NTC, NTD) - Variable changed from $\emptyset$ to 1 if conditions in note $A(B, C, D)$ which cause modification of a safety parameter value are satisfied.

25. P - Array containing the parameter values and the current value of the sort parameter. The first row contains the first parameter's values, etc.

26. PFLAGS - Asterisks placed next to paramater values that will be zeroed out due to special cases.

27. PN - Array containing the names of cards 2-18.

28. Q - Same as $\mathrm{P}$ except that the last value of the sort parameter is used.

29. R - Used in printing out the solutions and their safety sums.

30. Sols - Array into which the values of configurations of safety parameters that are solutions are read and maintained for subsequent reference.

31. SUMA - General safety computed for the current combination.

32. SUMA1 - Same as SUMA but uses the last value of the sort parameter.

33. SUMB - Containment safety computed for the current combination.

34. SUMB1 - Same as SUMB but uses the last sort parameter value.

35. SUMC - Extinguishment safety of the current combination.

36. SUMC1 - Same as SUMC but using the last sort parameter value.

37. SUMD - People movement safety of the current combination.

38. SUMD1 - Same as SUMD but using last sort parameter value.

39. TI - Column headings of the parameter values of the solutions. 
40. TITLE - Array containing the title to be placed on each page.

41. TJ - Column headings used in the actual printing of the solutions.

42. U - Contains the character "U" for flagging unprotected values.

43. UNPROT - The number of unprotected construction values.

44. X - Array, the minimum requirements read in.

45. $X M$ - Array, the maximum requirements read in. 


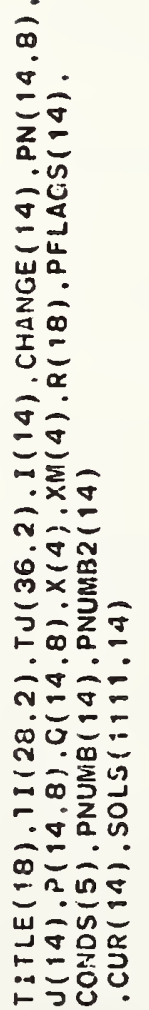

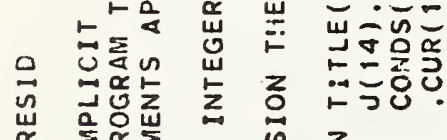

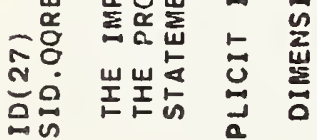

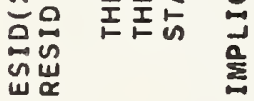

幽京

舟品

罗它

운

苮

学.

ज这

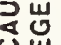

z

วิำ

㟧

은

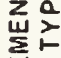
แ.

安岗皆 5 "아영

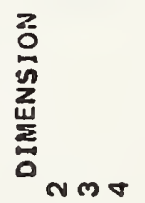

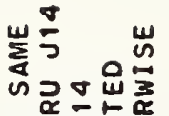

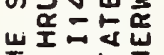

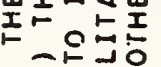
응

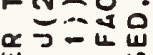

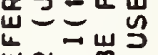
$\underset{\propto}{w}=\infty$ 응 $=0$ 아원명응

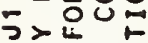
的金㟧

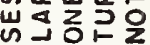
造的可 的些哭哥

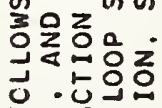
$4=4<$

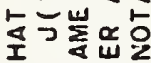
的的岁

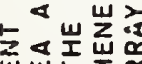

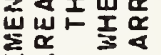

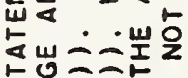
的最类的

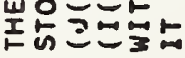

$\stackrel{0}{0}$
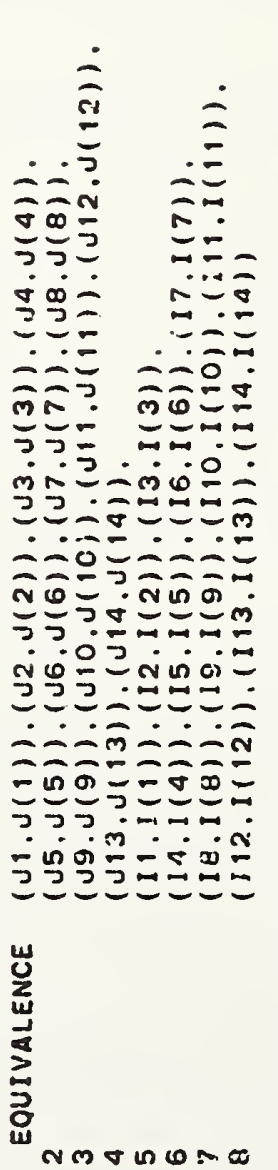

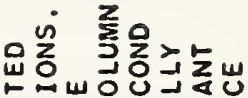

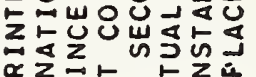

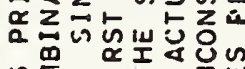

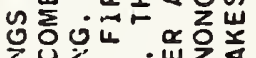

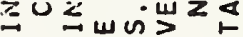

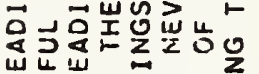

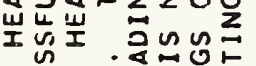

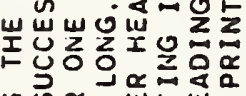
ज以屈

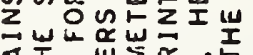

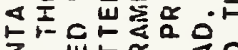

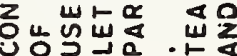

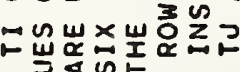

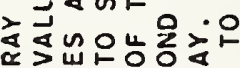
足 \&出水㟧品

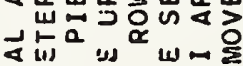

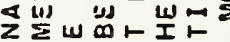

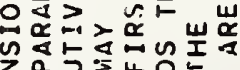

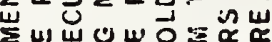

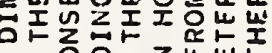

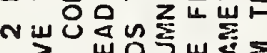
山。이몰

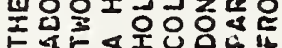

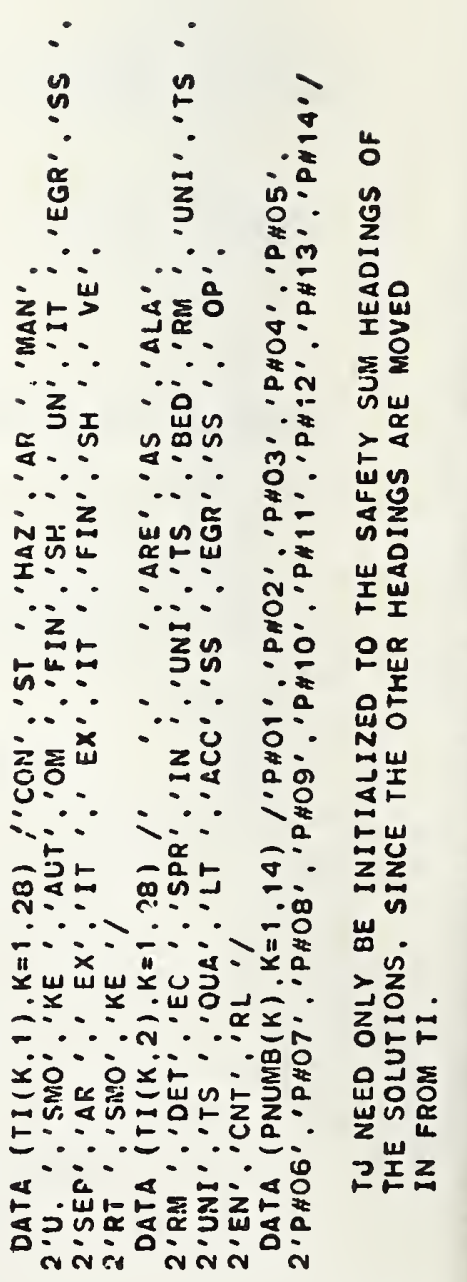

00000

$\overline{0}$

in

- ำ 


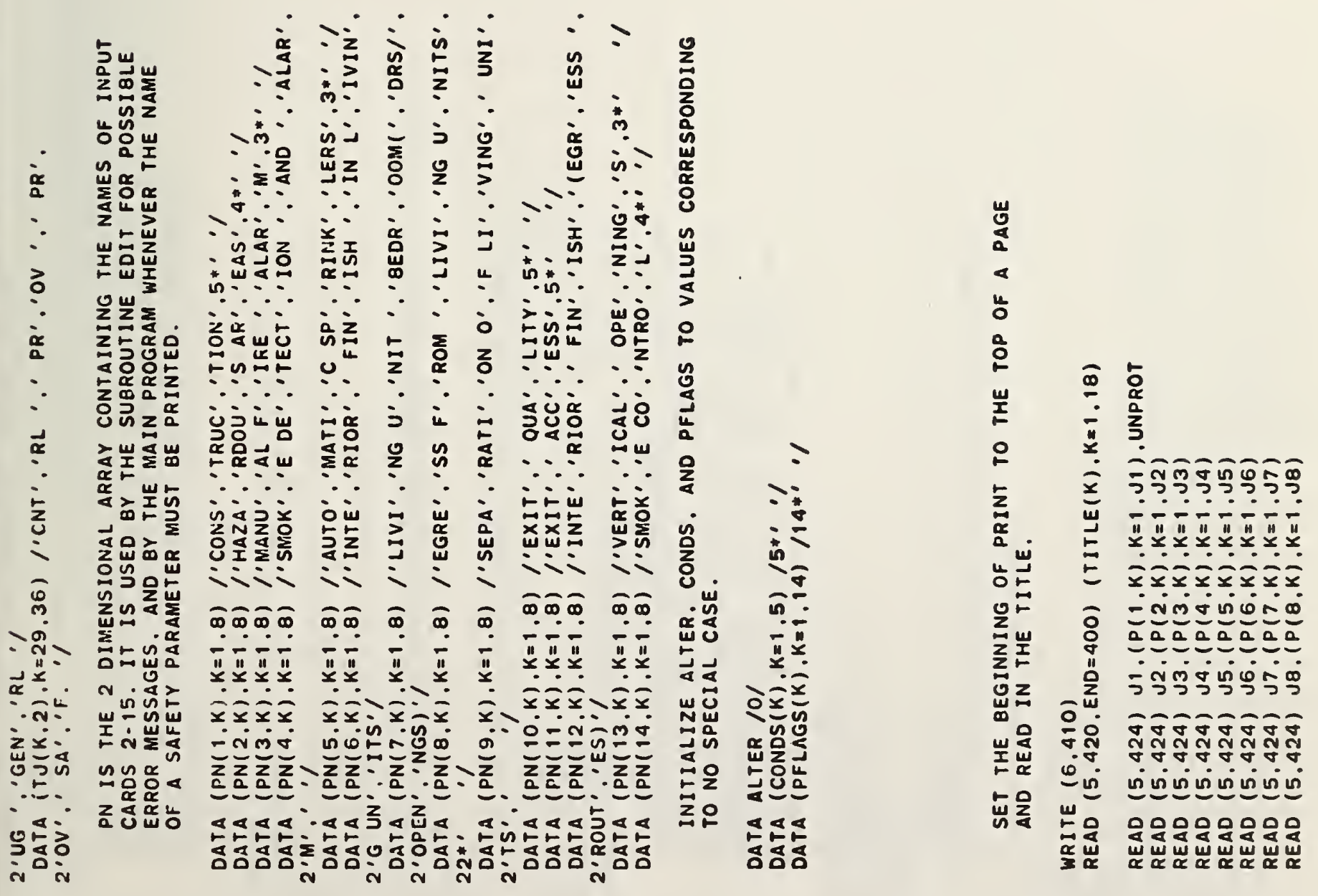

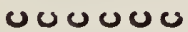

$\cup \circlearrowleft \cup \omega$

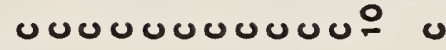

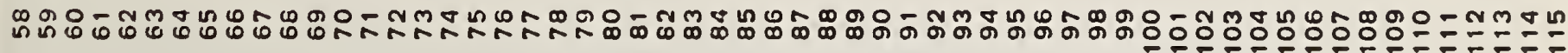



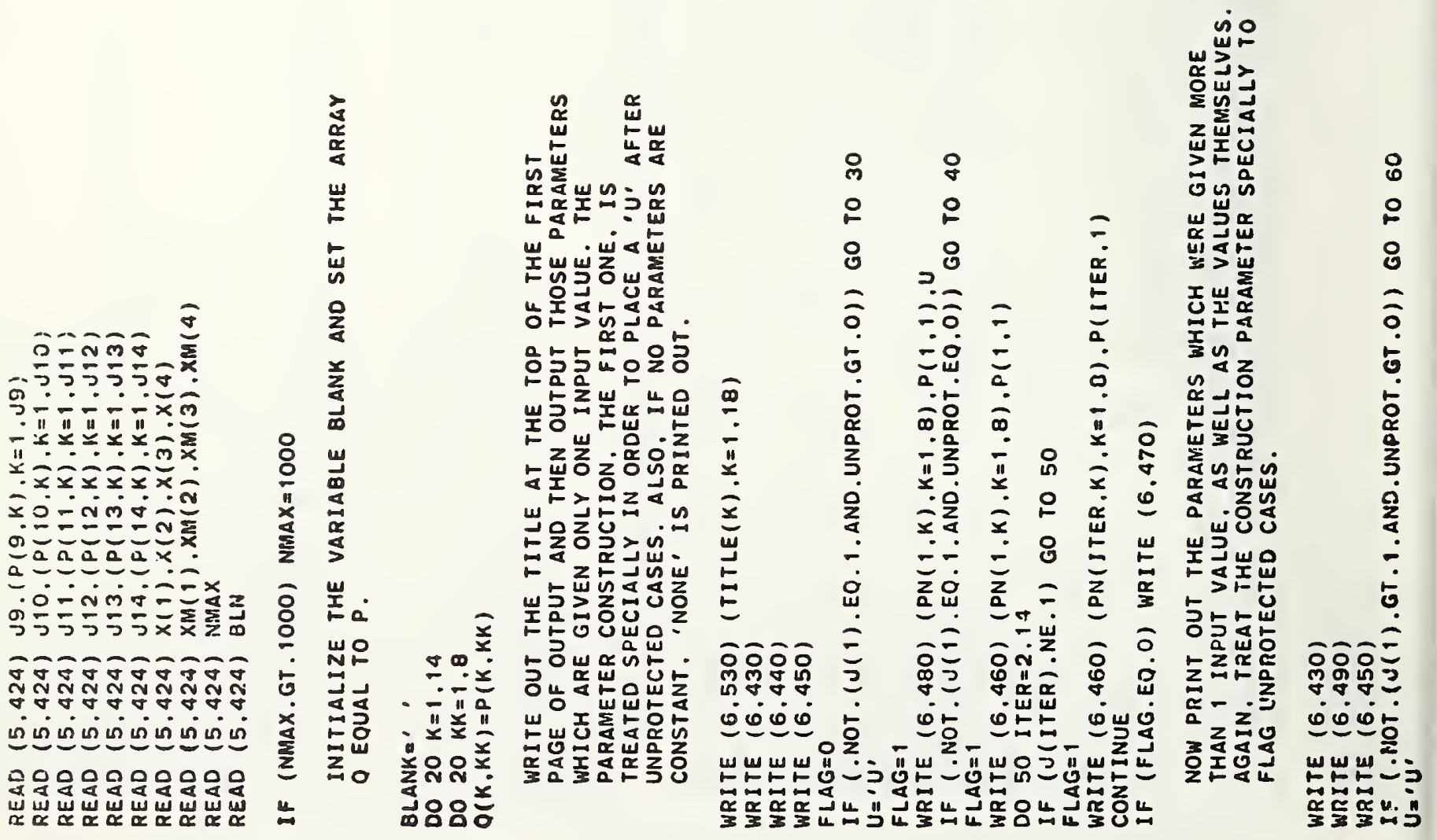

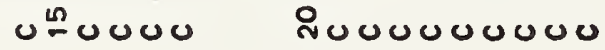

요

\&

000000 


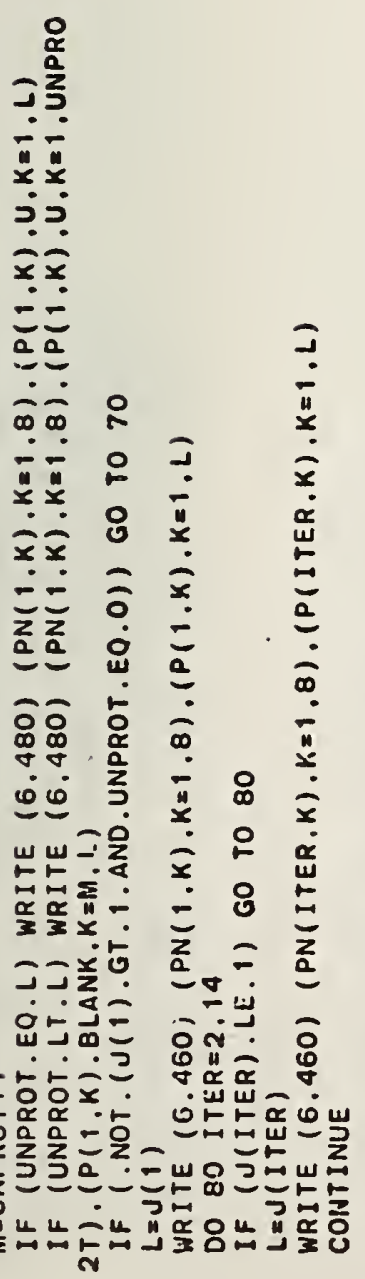

\&: §uovo $\sum_{\bar{\infty}}$

离

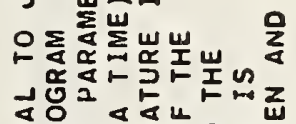

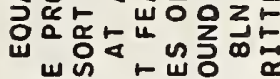

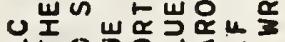

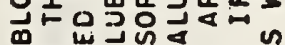

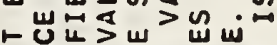

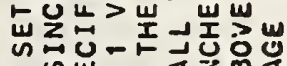

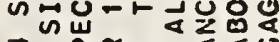

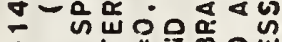
-

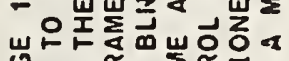
पू

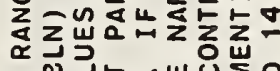

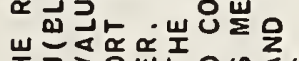

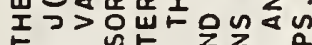

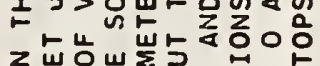

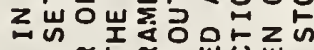

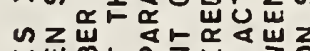

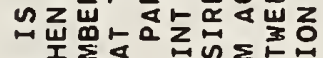
3 क

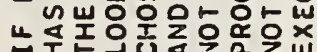

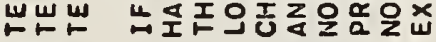
至 艝采采

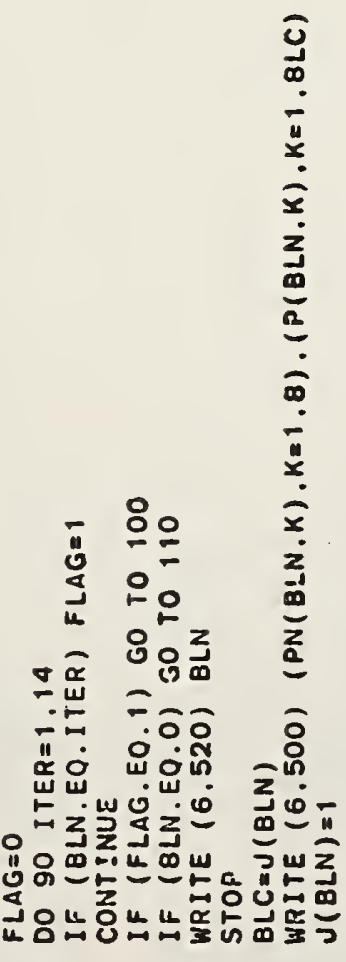



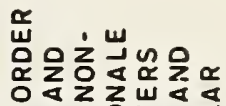

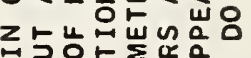

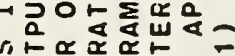

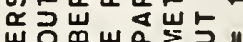

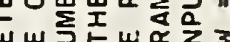

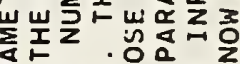

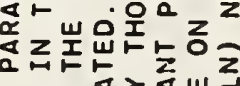

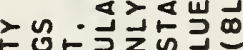

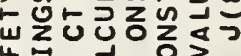
范.

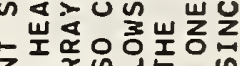

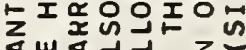

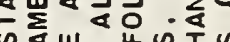

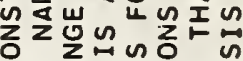

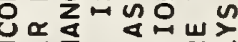
出的与器

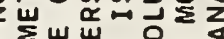

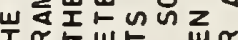

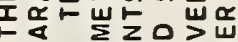

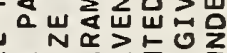

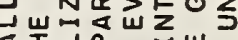

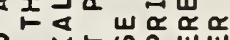

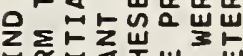

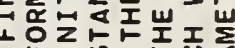

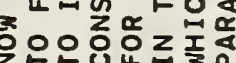

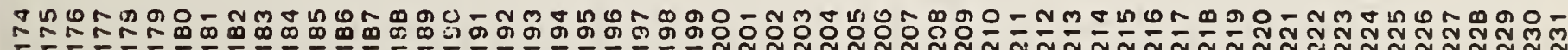



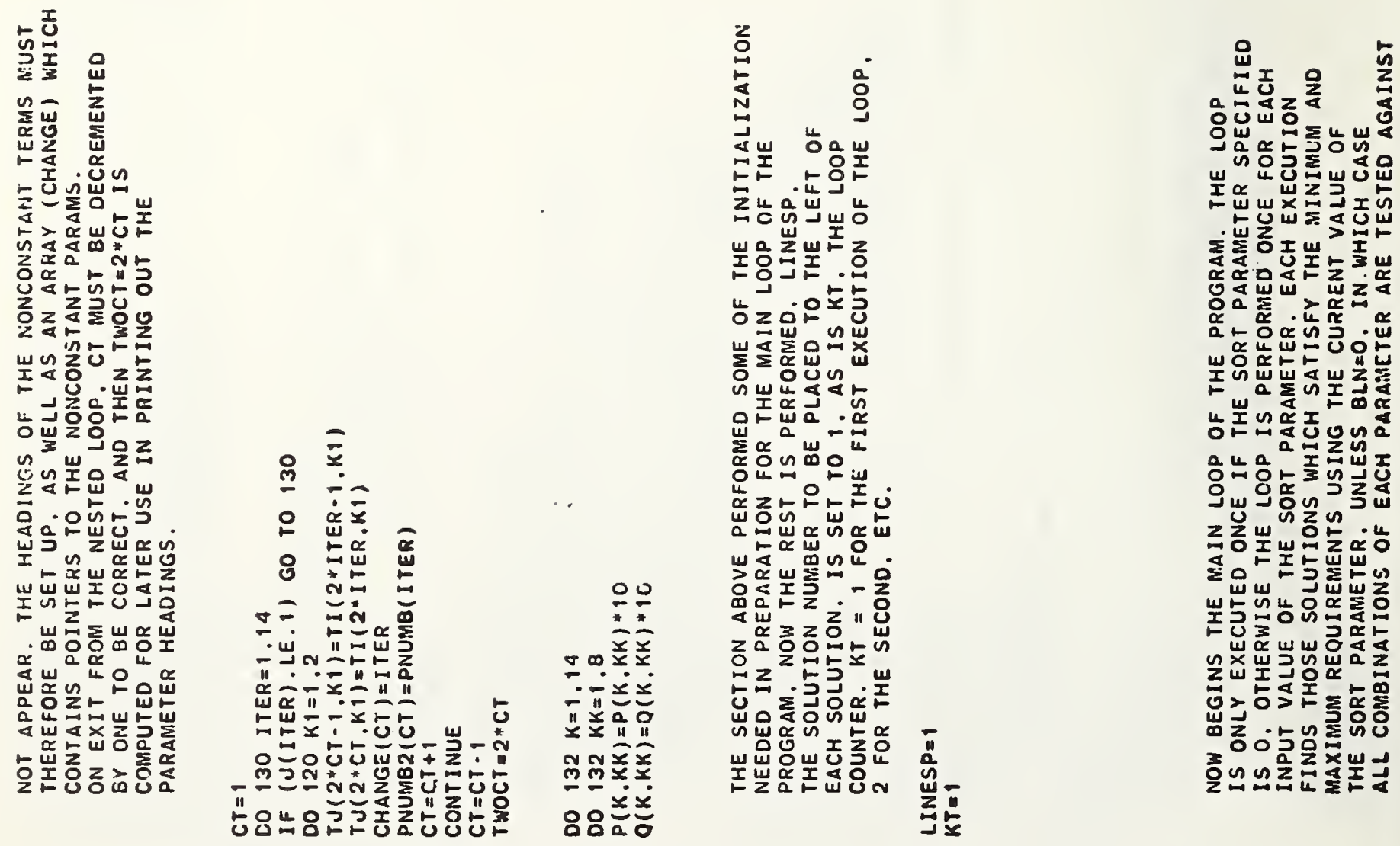

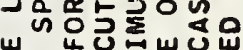

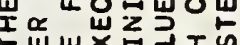

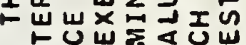
: 崖员IU 运出出

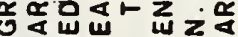

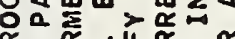

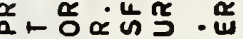

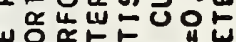
エ - a v

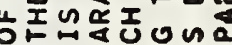

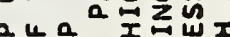
oun

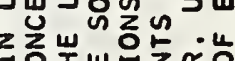

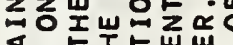

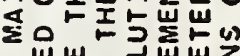

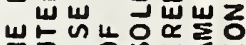
논

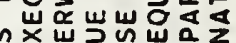

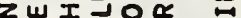
0>5ั

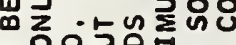

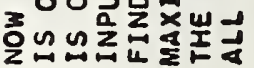

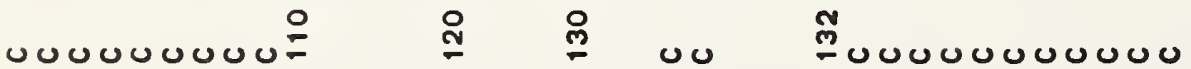

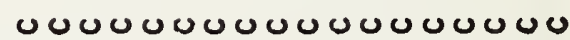

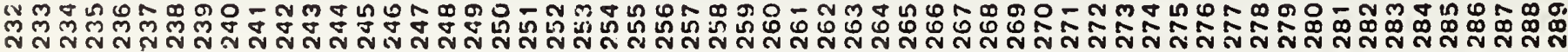




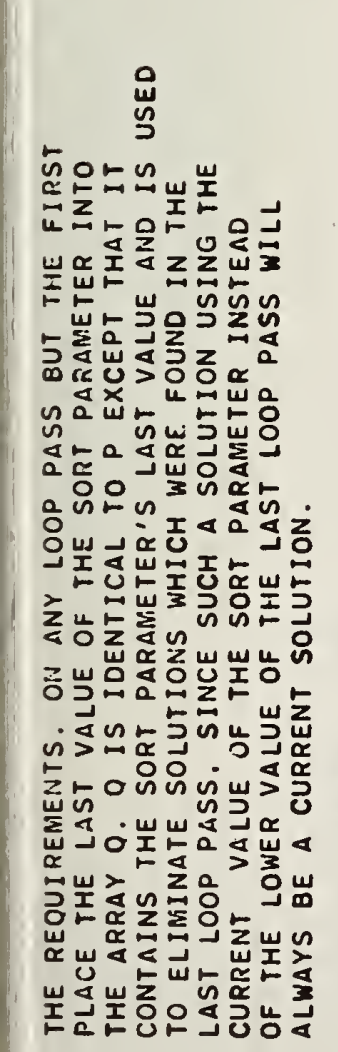

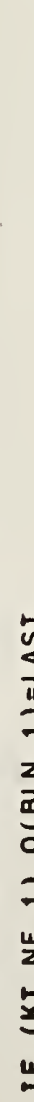

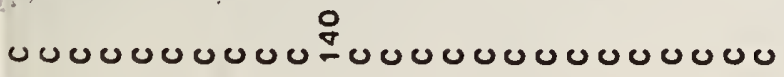

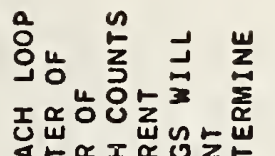

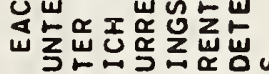

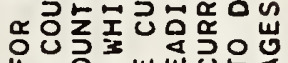

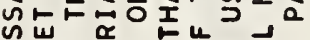

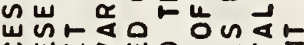
岀楸>出욛ㄴ

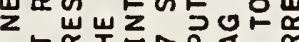

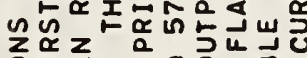

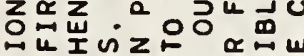

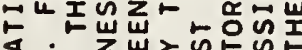

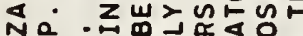

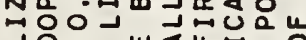
工

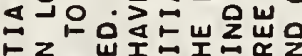

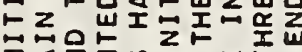

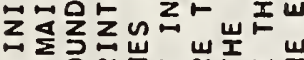

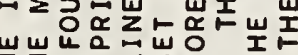

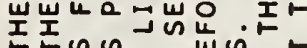

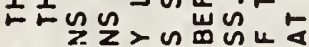

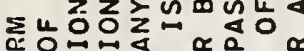

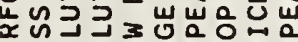

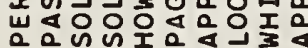

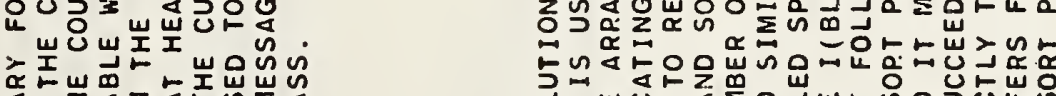

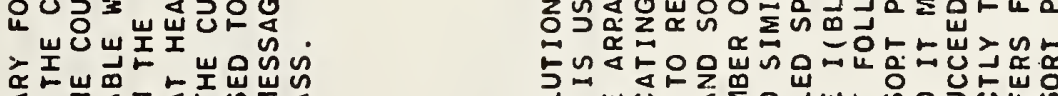

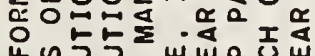

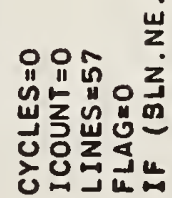

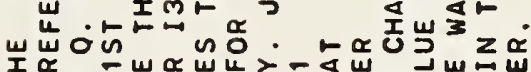

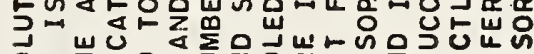

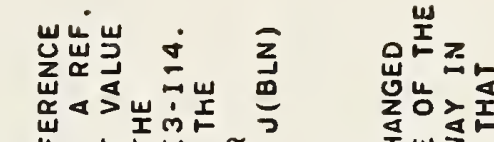

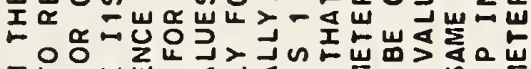

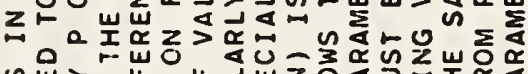

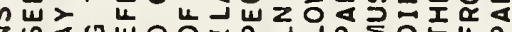

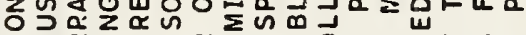

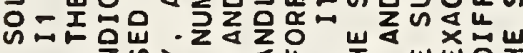

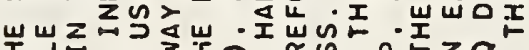

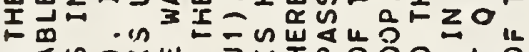

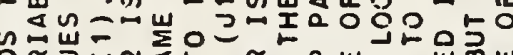

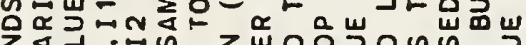

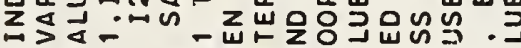

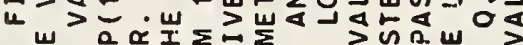

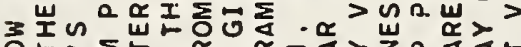

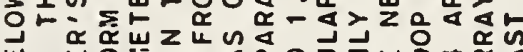

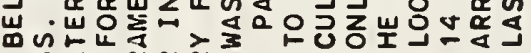

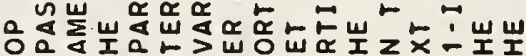

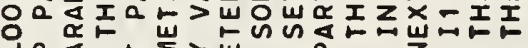

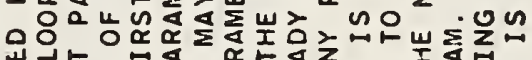
ज山山.

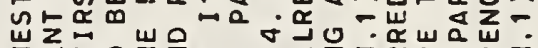

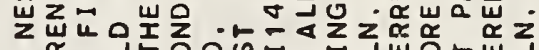

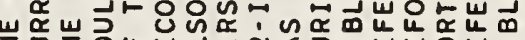

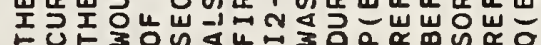

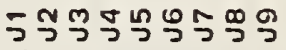
"

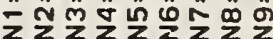

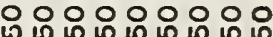

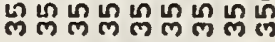

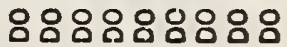

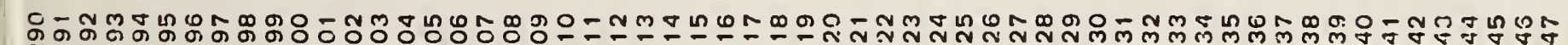

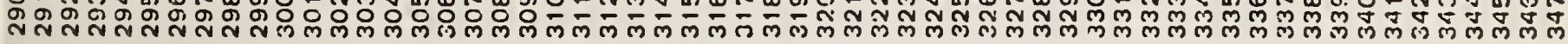




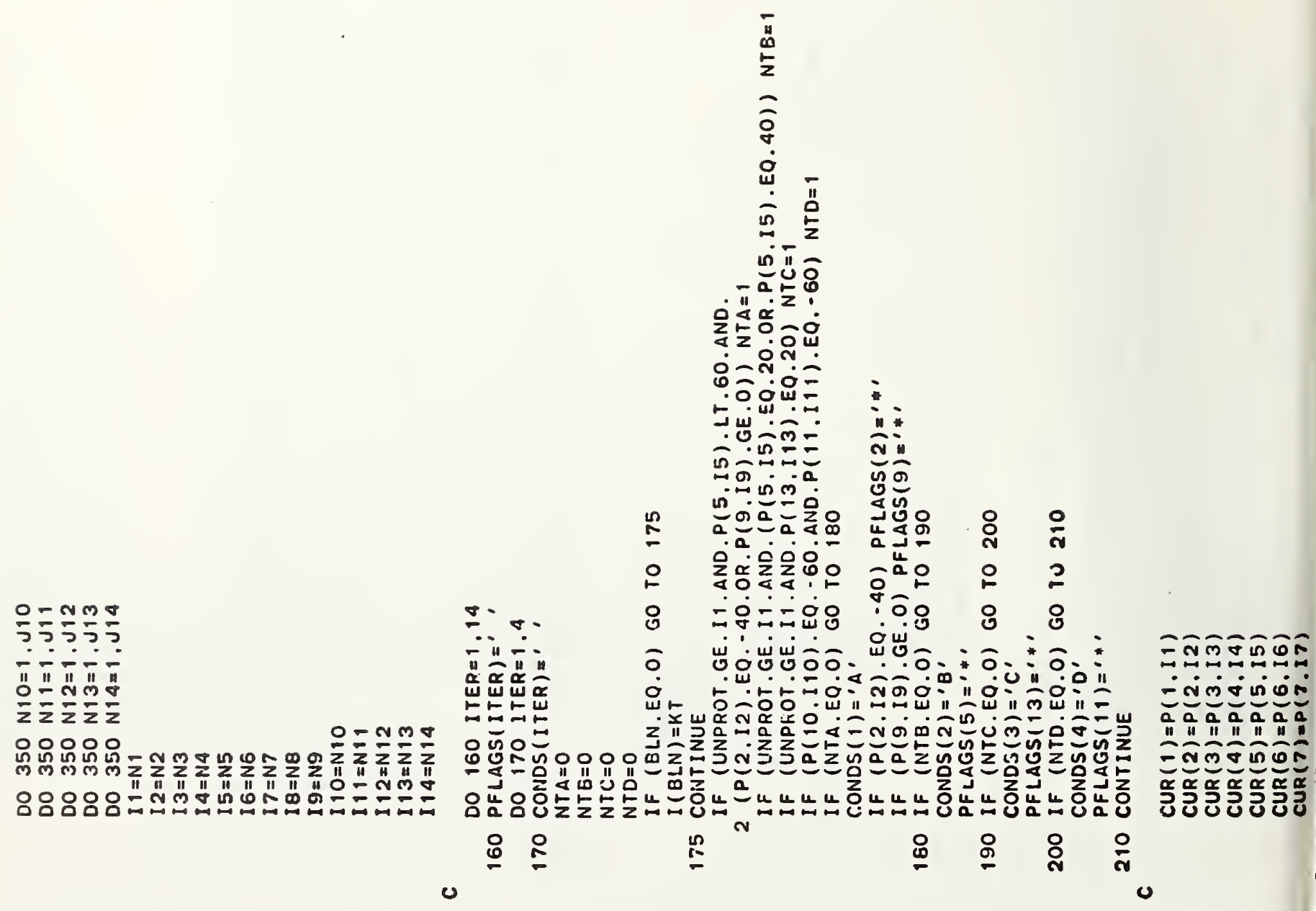

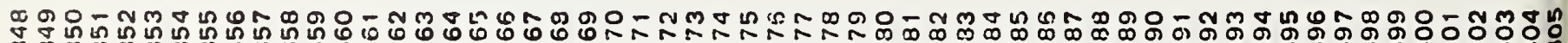

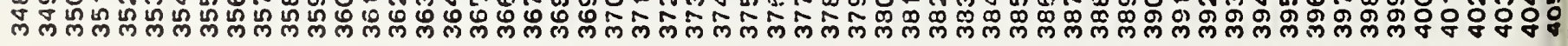




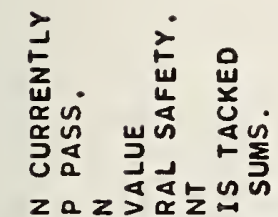

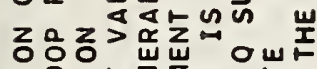

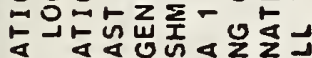

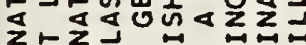
저에

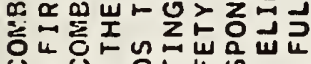

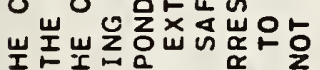

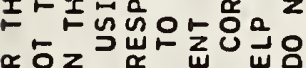

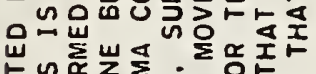

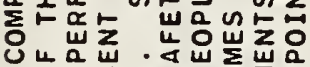

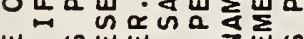

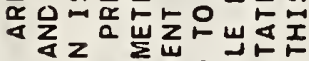

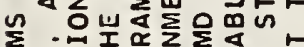

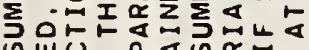

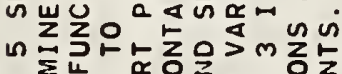
ш

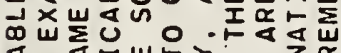

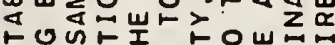

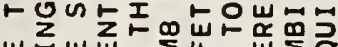

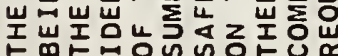
のこニこここ -

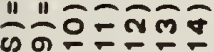
togect

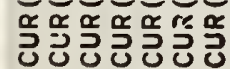

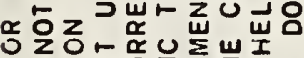

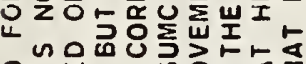

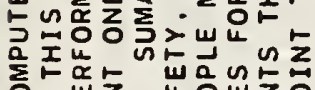

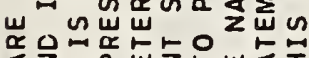

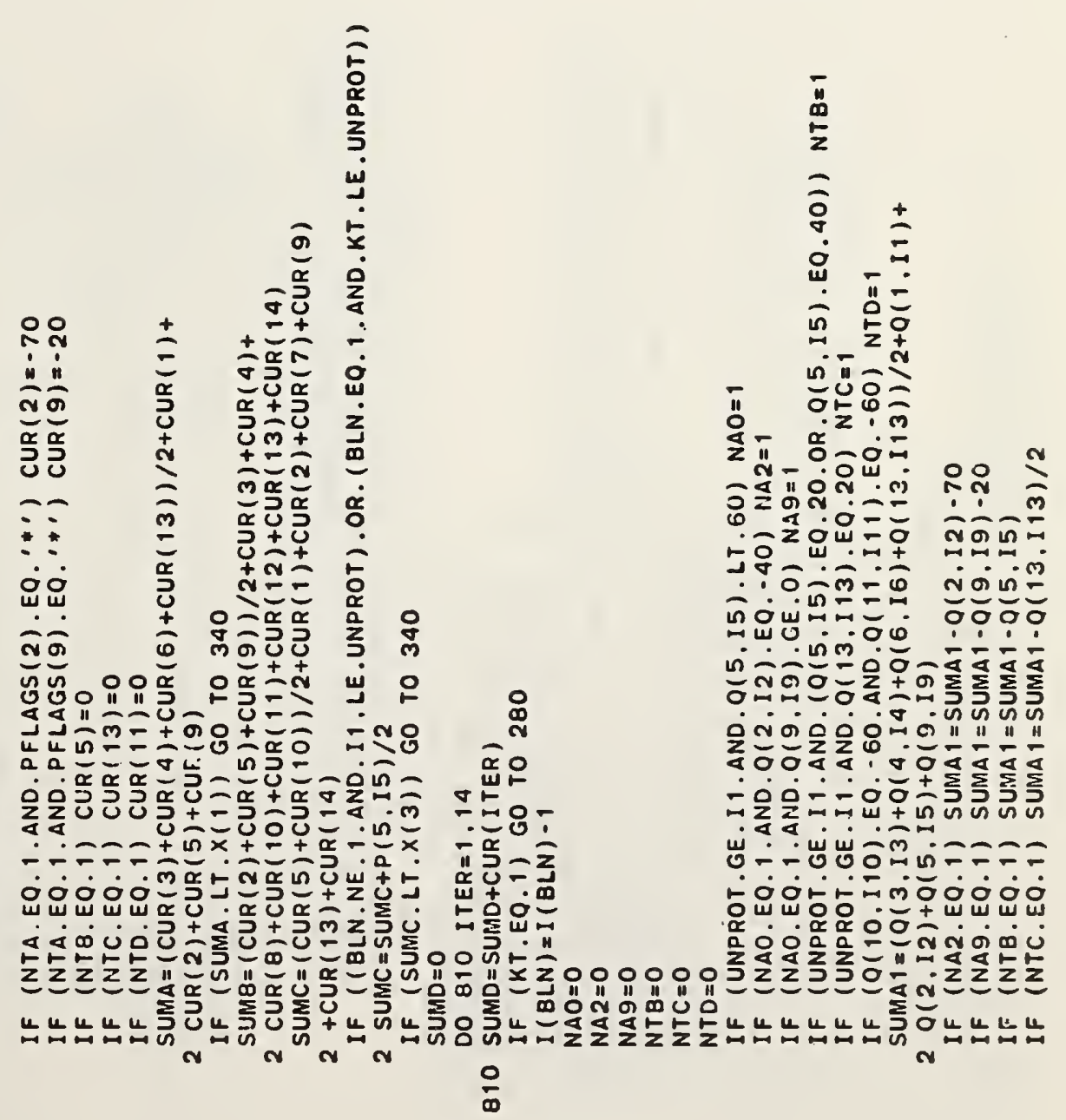

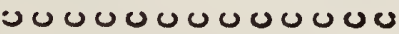

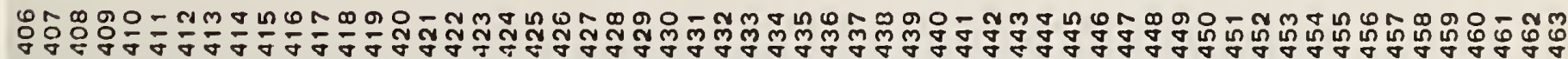




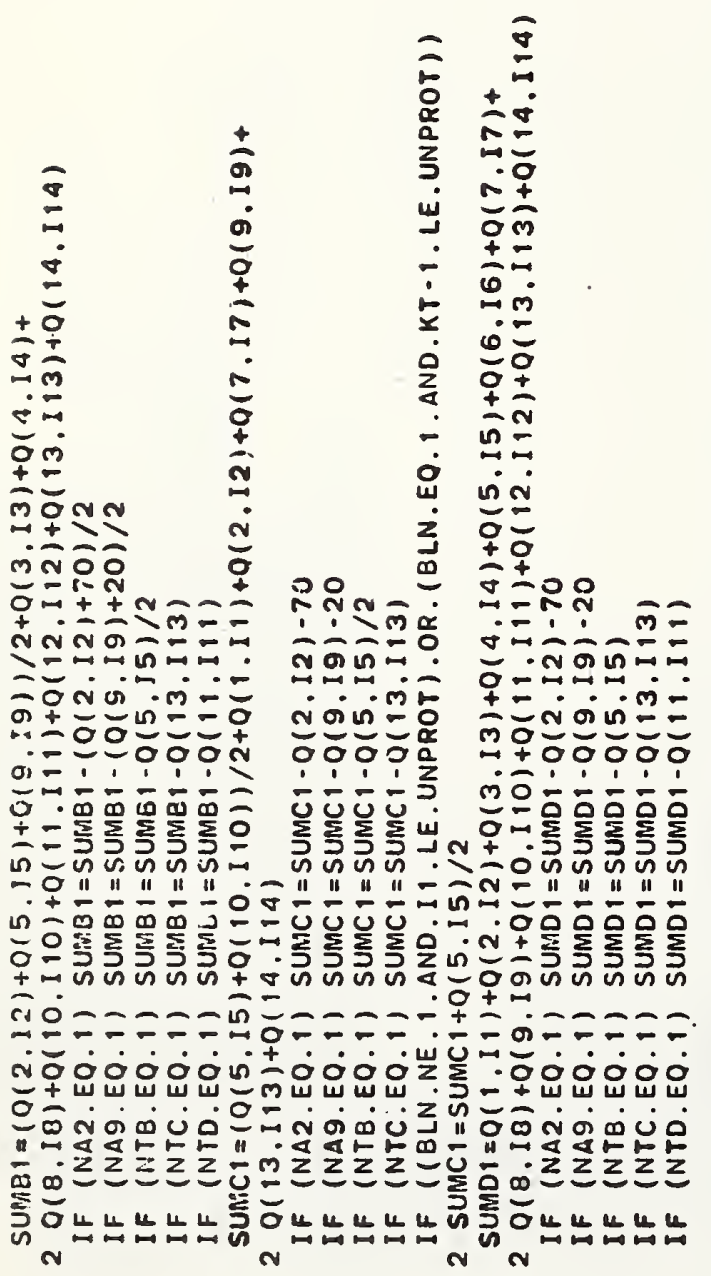

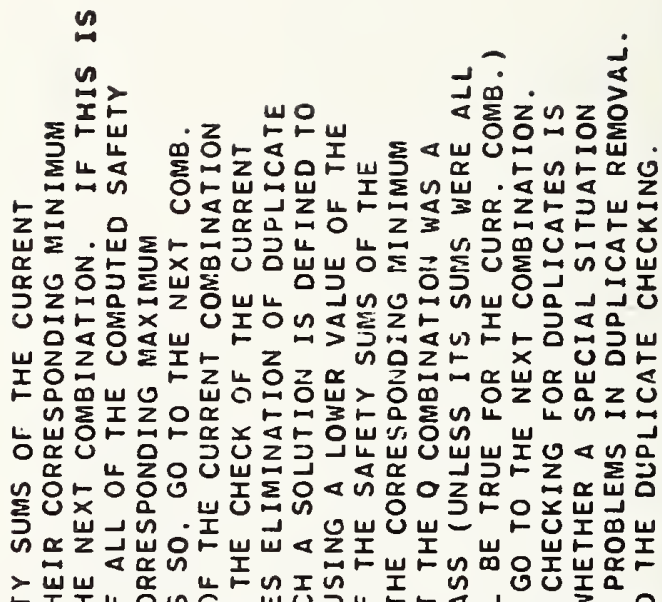

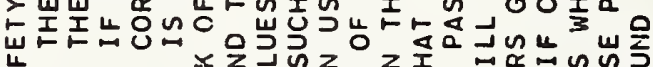

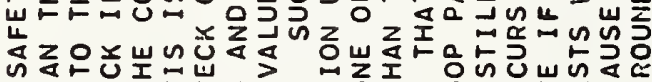

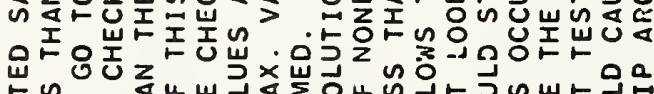

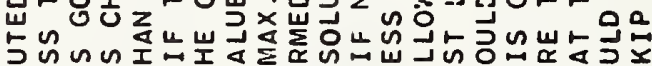

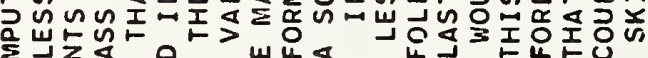

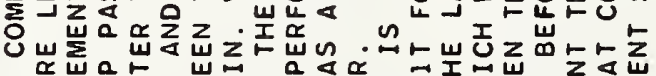

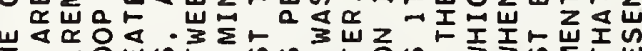

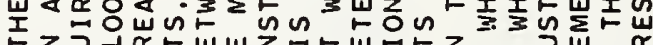

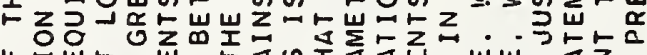

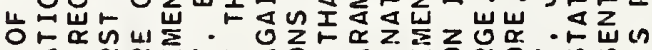

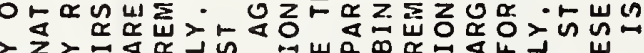

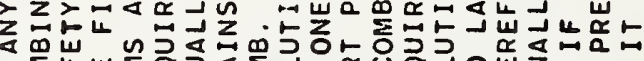

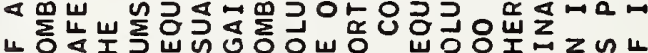

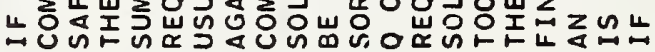

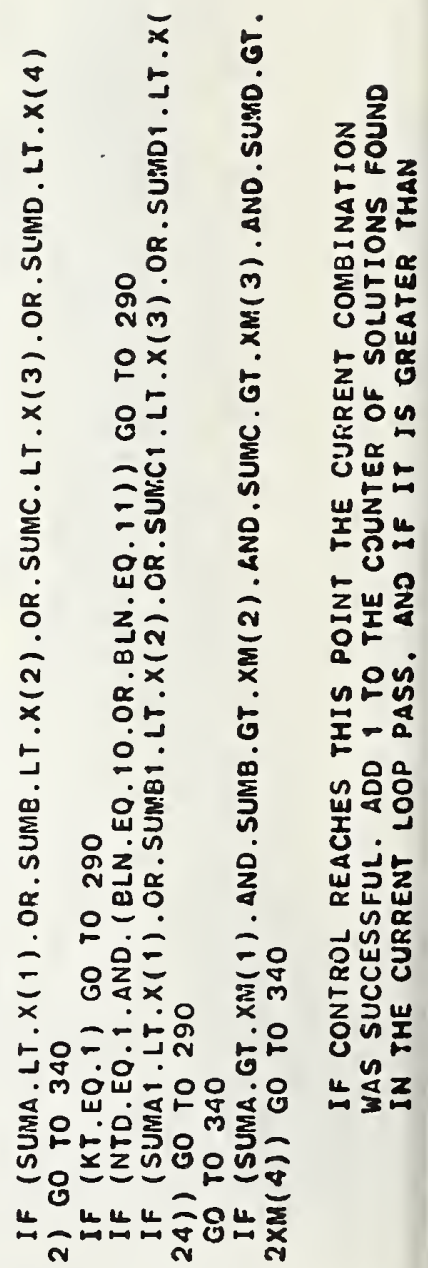

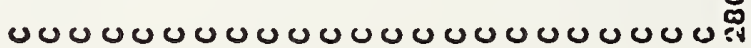

\&

טu०o

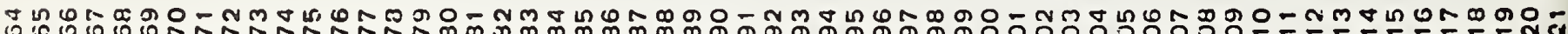

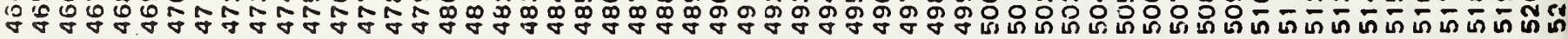




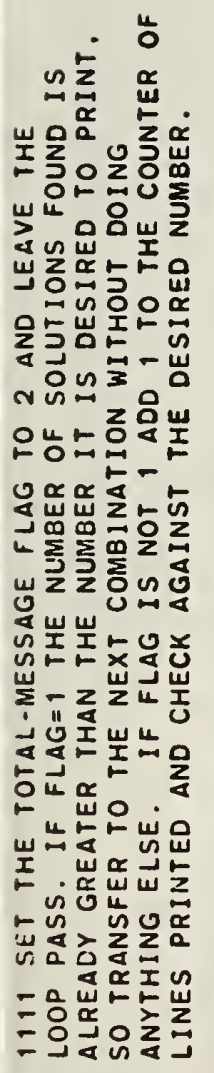

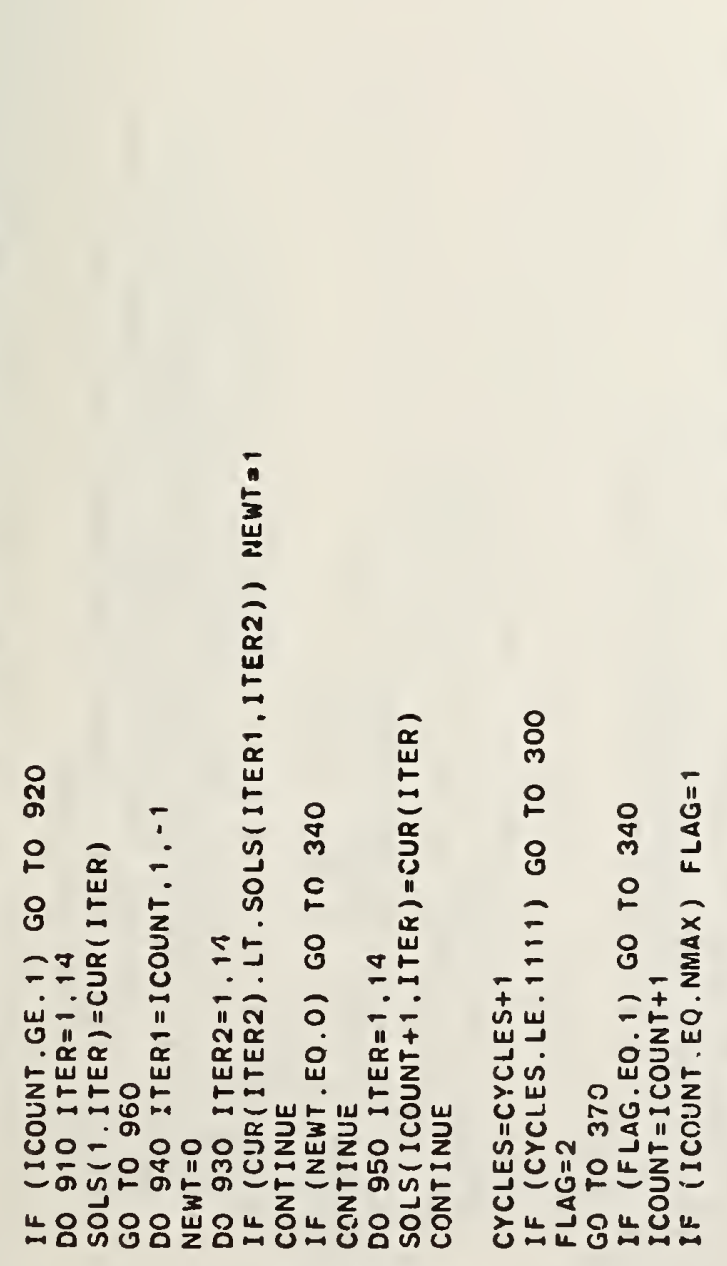

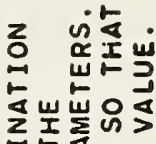

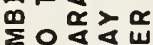

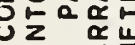

,$\frac{2}{2}$

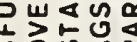

ino

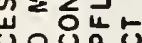

언은먼

굴일

w्.

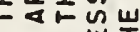

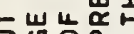

응

폰

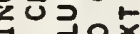

z

z

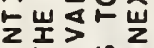

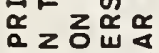

은

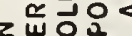

20

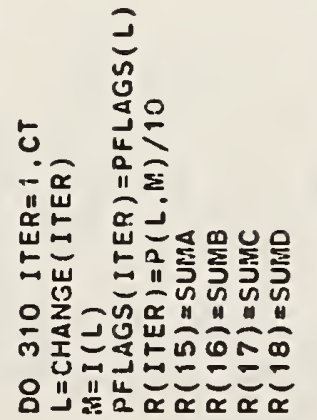

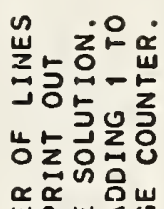

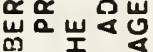

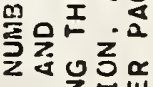

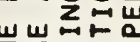

崎

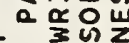

似

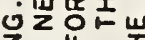

Zw㟧上I

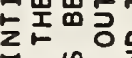

중원

$\div \geq$

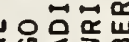

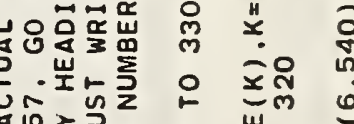

的器改

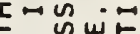

山心山?

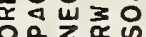

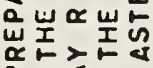

a

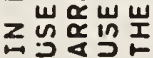

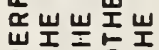

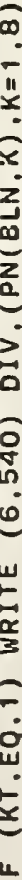

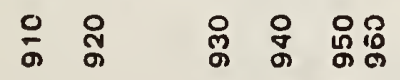

:

응

UUU⿻上丨

곡

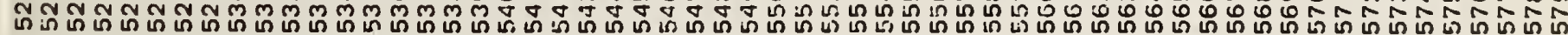



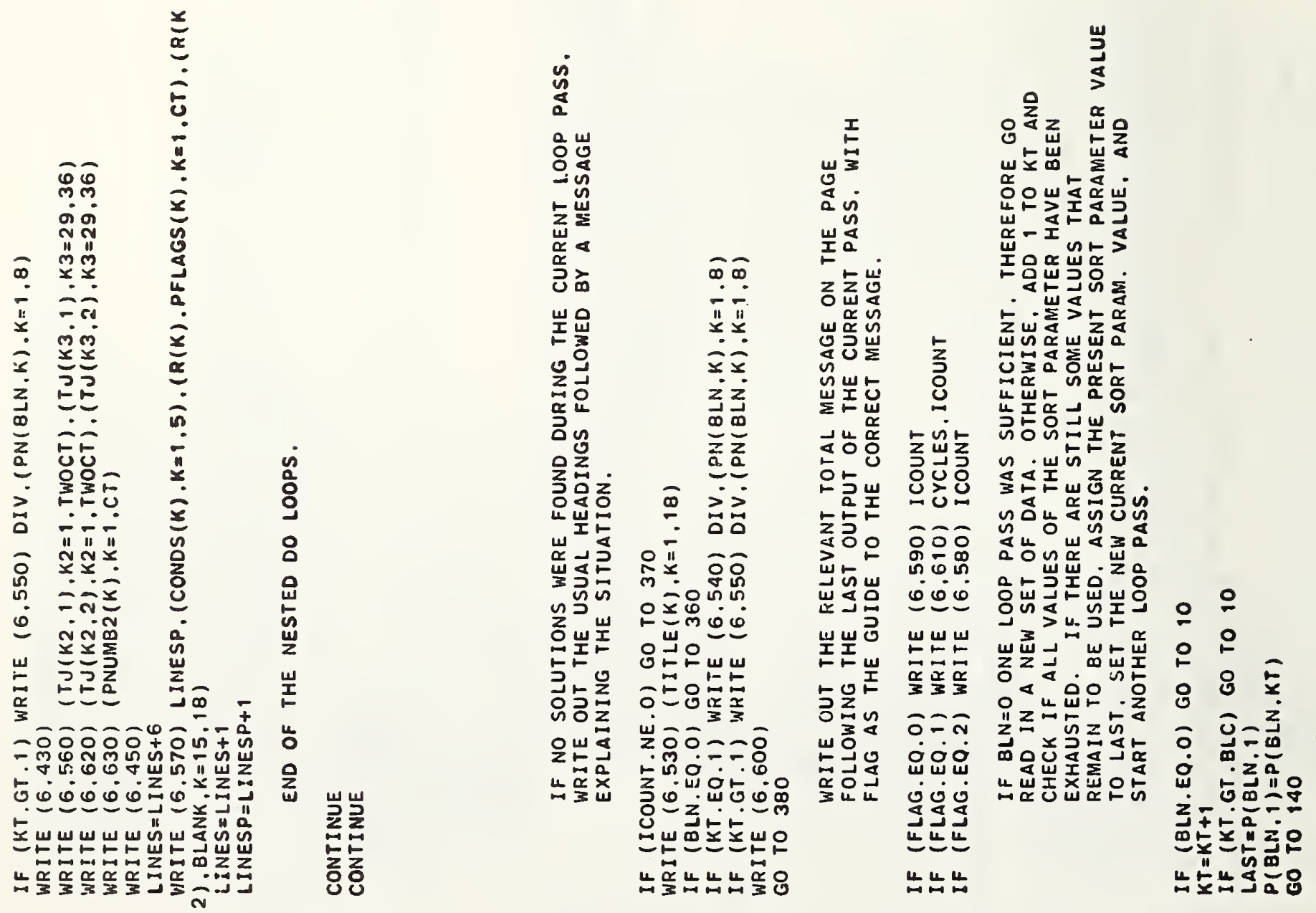

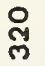

$\because$

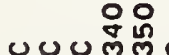

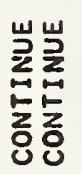

:000000

竞

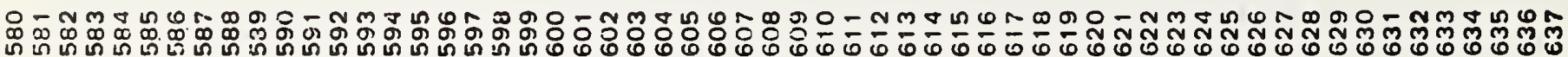




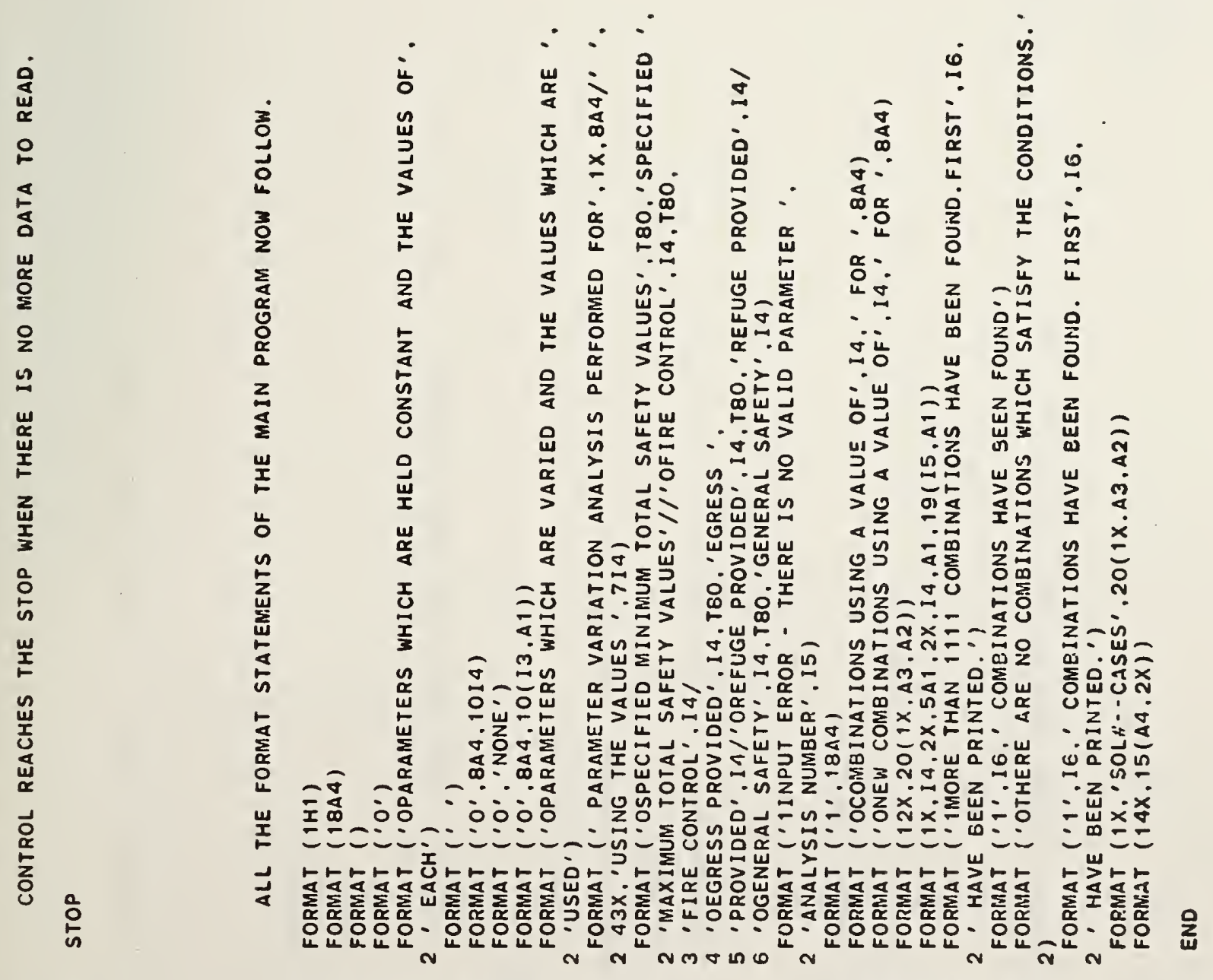

u

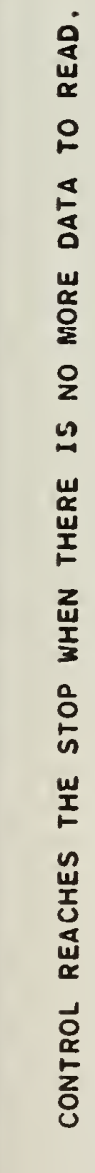

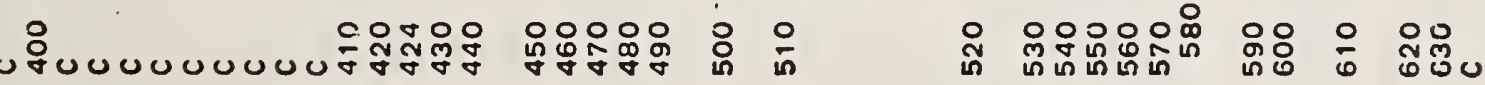

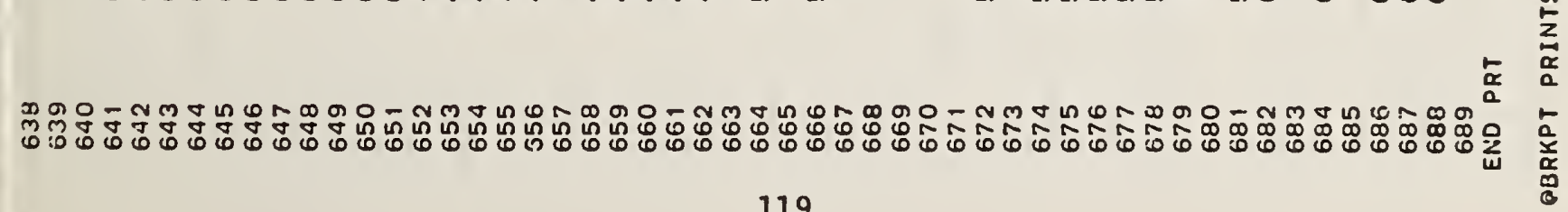


Example 1

\begin{tabular}{|c|c|c|}
\hline 1 & 2 & \multirow[t]{2}{*}{0} \\
\hline 1 & 0 & \\
\hline 2 & -1 & 2 \\
\hline 1 & 0 & \\
\hline 1 & 2 & \\
\hline 2 & -1 & 0 \\
\hline 2 & 0 & 1 \\
\hline 2 & 0 & 1 \\
\hline 2 & 2 & 4 \\
\hline 2 & -6 & -2 \\
\hline 3 & -2 & 0 \\
\hline 2 & -1 & 0 \\
\hline 2 & 0 & 2 \\
\hline 2 & 3 & 4 \\
\hline 0 & 0 & 0 \\
\hline 90 & 90 & 90 \\
\hline 00 & & \\
\hline
\end{tabular}

Example 2

$\begin{array}{llll}2 & -2 & 2 & 1\end{array}$

$\begin{array}{lll}2 & -4 & 0\end{array}$

$\begin{array}{lll}2 & -1 & 2\end{array}$

$\begin{array}{lll}2 & 0 & 2\end{array}$

$\begin{array}{lll}2 & 0 & 2\end{array}$

$\begin{array}{lll}2 & -1 & 0\end{array}$

2001

$\begin{array}{lll}2 & 0 & 1\end{array}$

$\begin{array}{lll}2 & -6 & -2\end{array}$

$\begin{array}{lll}2 & -2 & 0\end{array}$

$\begin{array}{lll}2 & -2 & -1\end{array}$

$2 \quad-1 \quad 0$

$20 \quad 2$

200

$-100-100-100-100$

$60 \quad 60 \quad 60 \quad 60$

1000 


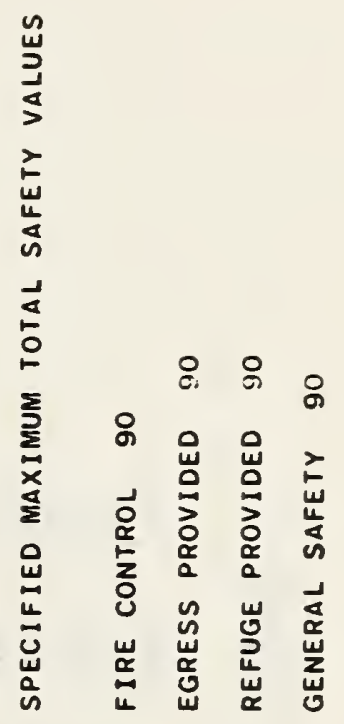

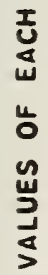

岸

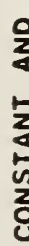

为 2000

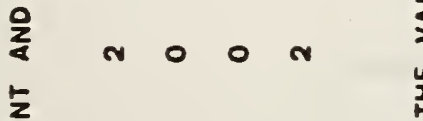

일

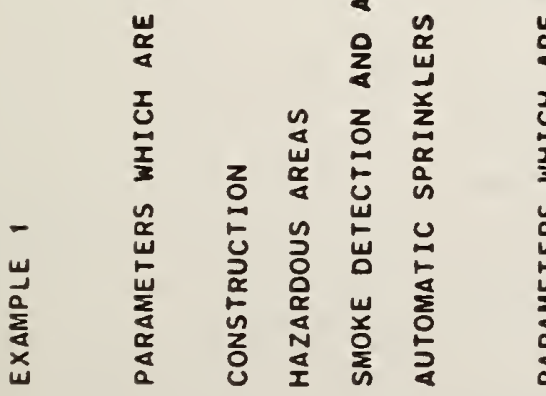

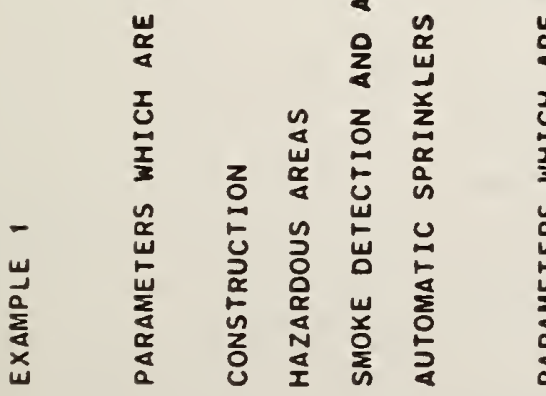

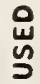

$\underset{\frac{\pi}{\alpha}}{4}$

疒 $\stackrel{\frac{\pi}{5}}{\frac{\pi}{4}}$

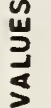

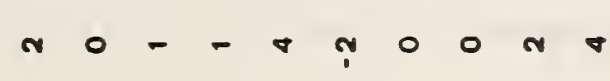

声

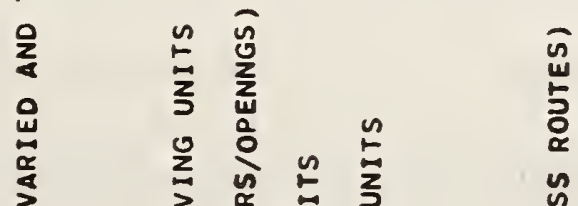

点

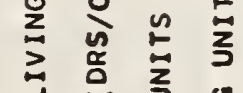

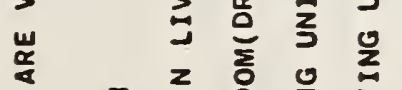

$\underset{u}{\tilde{u}}$

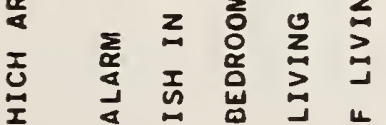

兽

$\stackrel{1}{5}$

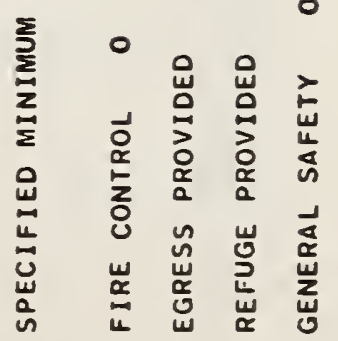




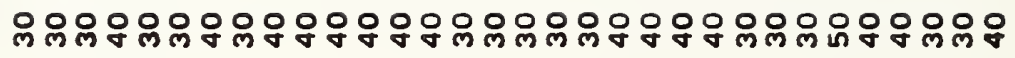

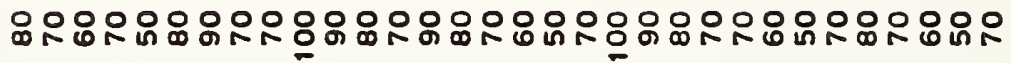
00000000000000000000000000800000

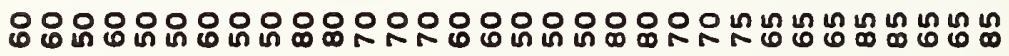

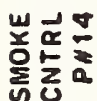

भ M

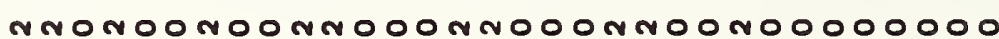

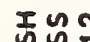
$\sum \frac{0}{4}$

$\mathfrak{n} \mathfrak{n =}$

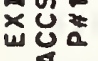

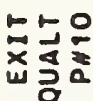

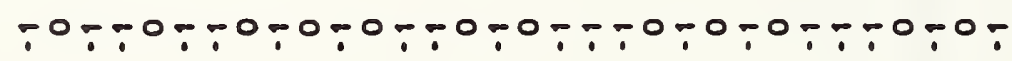

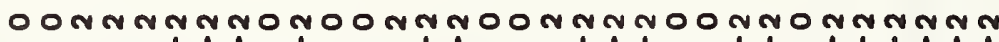

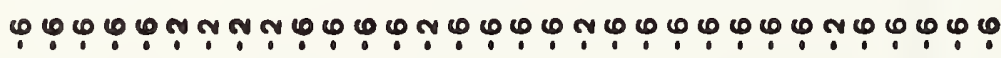
$\stackrel{\infty}{\infty} \rho$

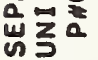

nu: $000000000000000-n-n-n 000000-n$ (5)

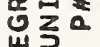

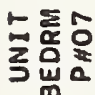

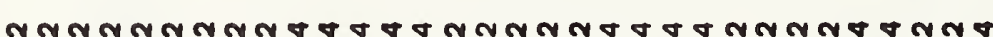
รัง $\sum_{\substack{u \\ \sum_{0}}}$ วi mo

00000000000000000000000000000000 


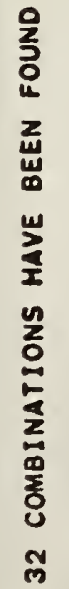




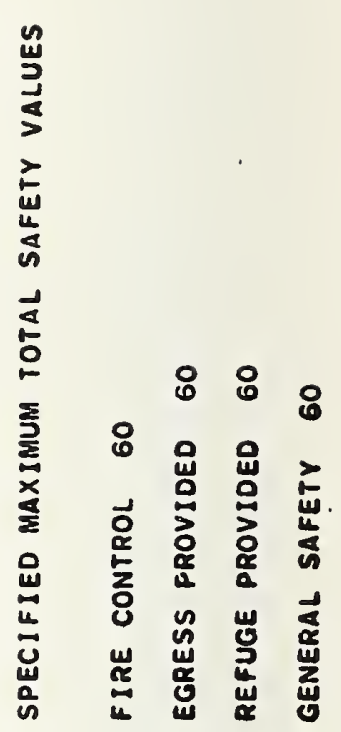

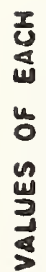

온

щᄄㅜㅇ

崖

울

妾

$\stackrel{9}{\underline{m}}$

$\underset{\alpha}{\alpha}$

돈

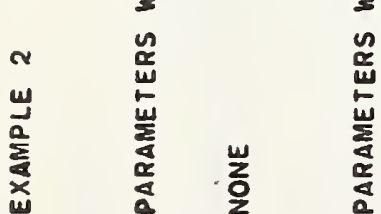

건

$\stackrel{\text { S }}{3}$

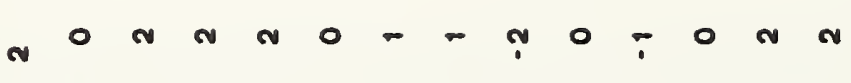

虫

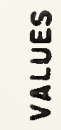

울

$\stackrel{5}{5}$

足<smiles>CCC</smiles>

峞

(3)

I

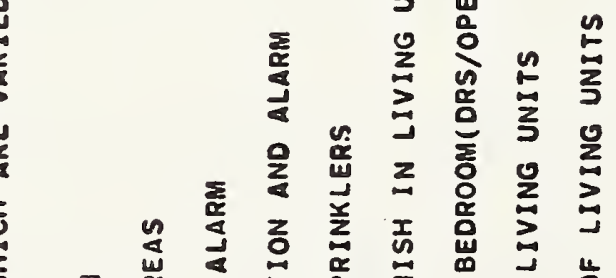

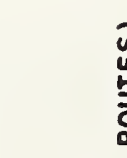

\section{点}

$\sum_{0}^{\frac{w}{4}}$

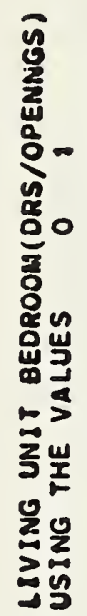

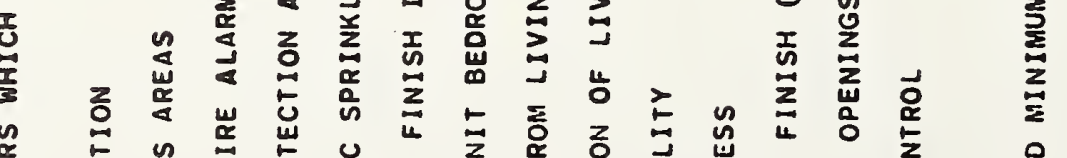

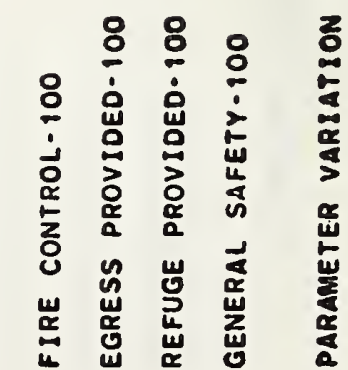




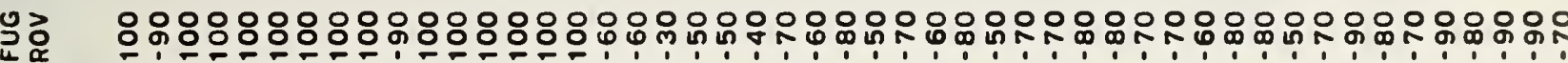

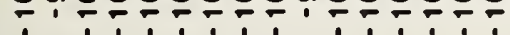

$n>$

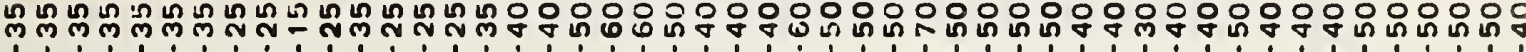

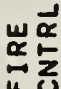

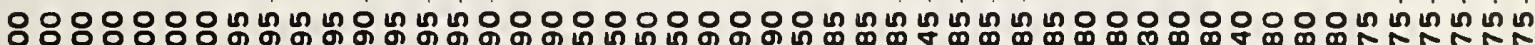

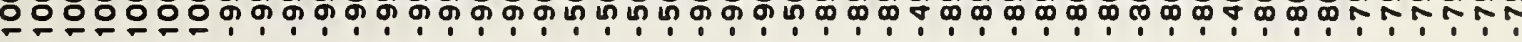

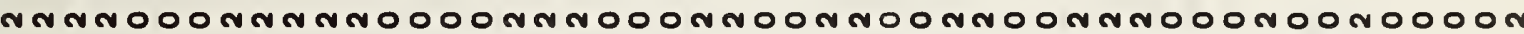

0000000000000000000000000000000000000000000000000

$0 \div 0 \div 00 \div 0 \div 5 \div 50 \div 5 \div 0 \div 50 \div 50 \div 0 \div 0 \div 0 \div 0 \div 0 \div 0 \div 50 \div 5 \div 0 \div 50 \div 0 \div 5$

u

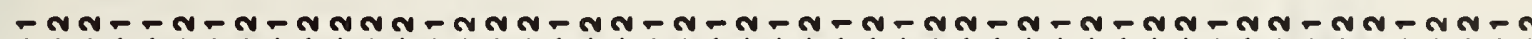

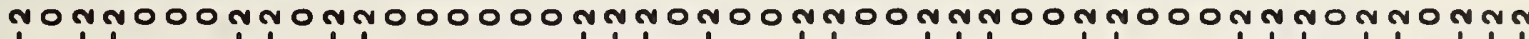
$\propto \sim g$

눙우

4 ג wวaㅁ

ตำ

นㄴㅇㅇㅇ

존은

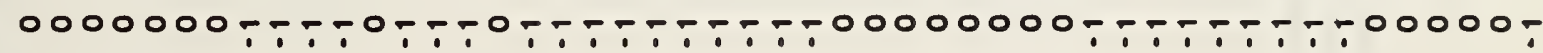
4.

son

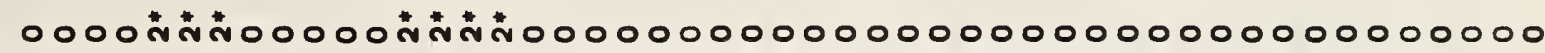
$m 00$

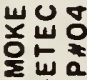

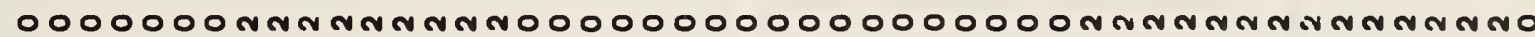
wa

○

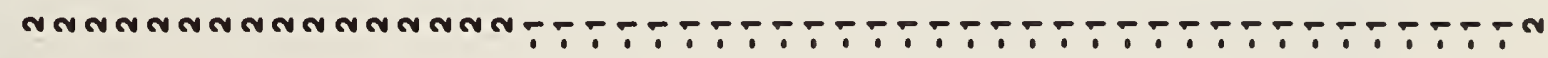

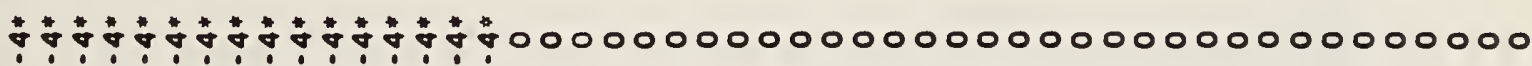

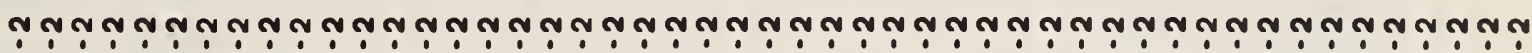
$n$
$u$
0
$\vdots$
$\vdots$
0
0

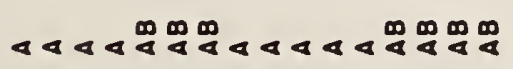

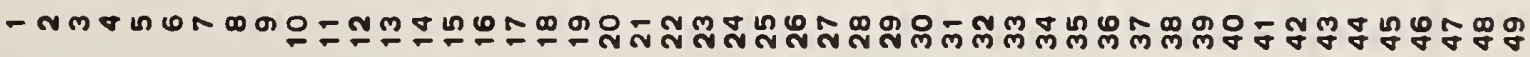




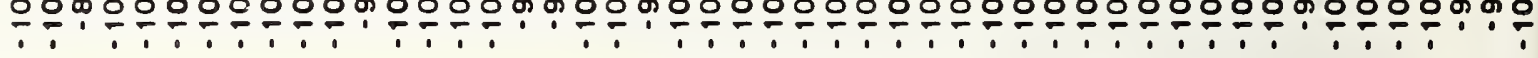

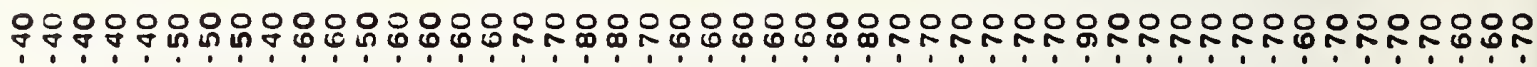

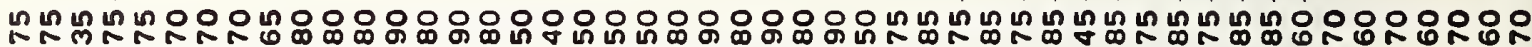

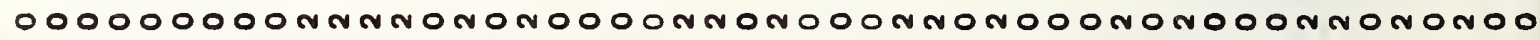

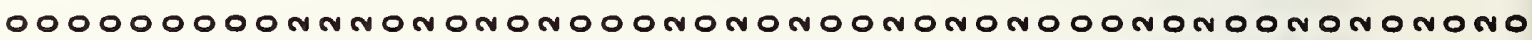

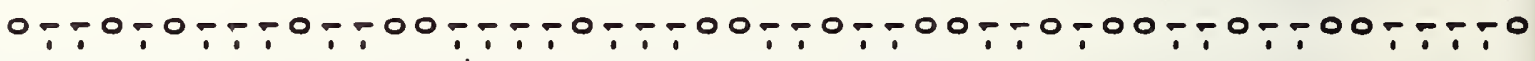

$\because \tilde{x}=$

$\underset{\omega}{x} \stackrel{0}{a}$

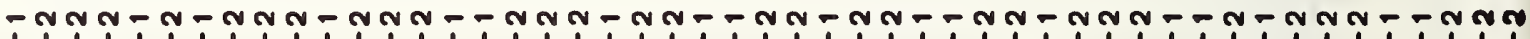

$\vdash \vdash 0$

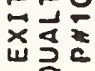

monnmw? $\propto \sim \sigma$

ato ù

$\backsim \backsim \infty$

$\stackrel{0}{0}=0$

는

Iே

$\sum_{\substack{n \\ \vdots}}$

홍릉요

둥응

넝무

๒ $000-500-0000000000000---5--0000000----500000000$

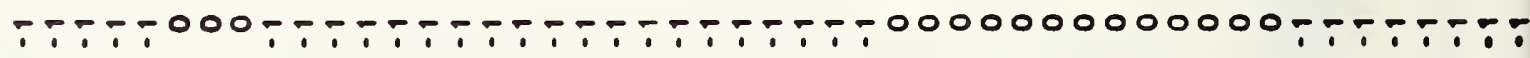
00000000000000000000000000000000000000000 nnnnnm 00000000 N0000000000000000000000000000000000000000 施

4n

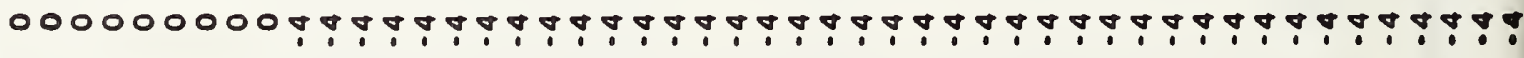

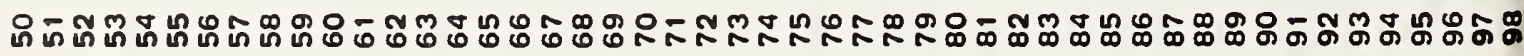


$\sum_{u}^{\alpha}$

궁 $\underset{\alpha}{\alpha}$

«ㄱ응 0

$\underset{\alpha}{\alpha} \frac{\vec{\alpha}}{2}$

씸ㅁ

인운

zm

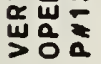

돈

芯恕。

-n-

떠ㅇㅝㅜ

トレ0

牙骂

뚀

450

岁资新

ทn

นั응

额金

亦的

는웅

릉응

눈

แบํํ

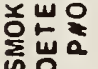

$\frac{1}{\circ}$

ऐ웡웡

ㄴ $N$

중ํํ

중우

능

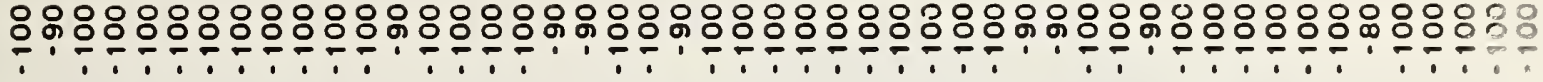

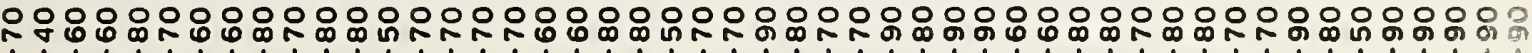

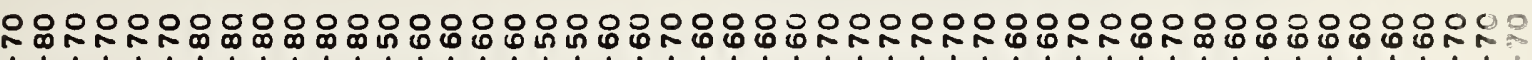

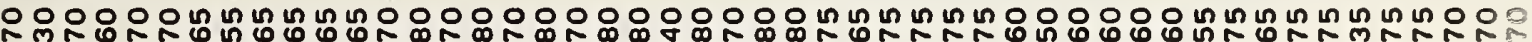

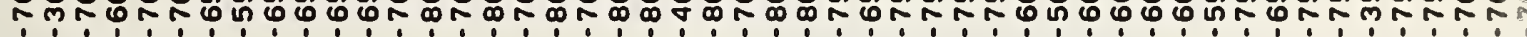

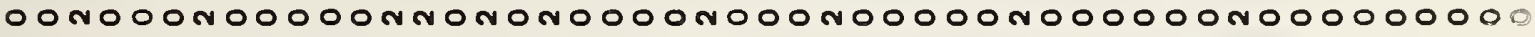

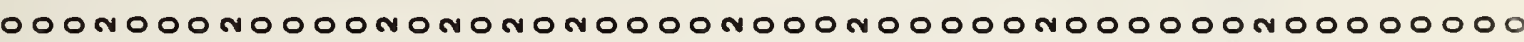
:

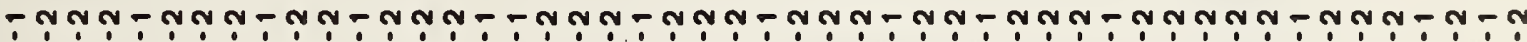

0

๑ $00---0000--0000000000--5-0000--00000-000000--00-$

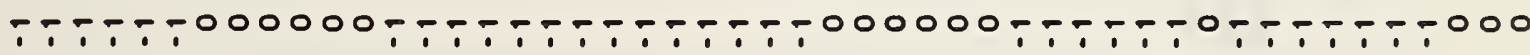

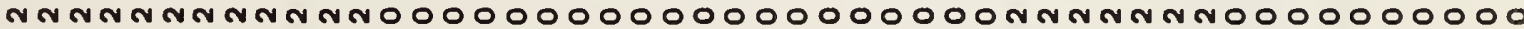

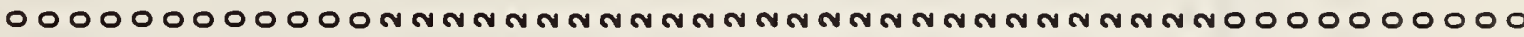

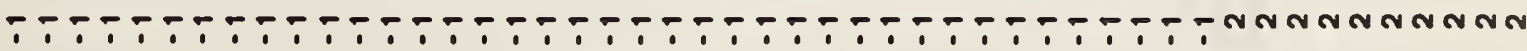

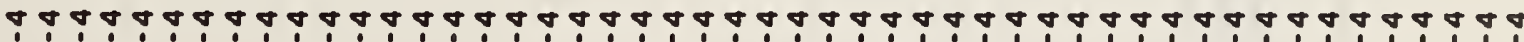

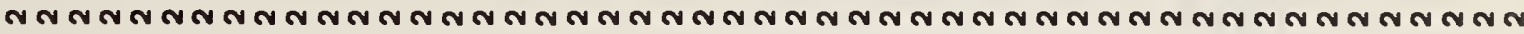

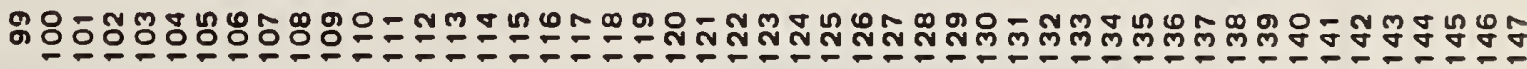




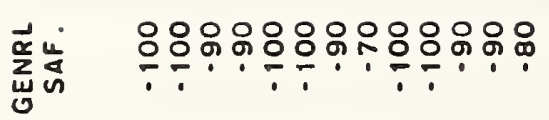

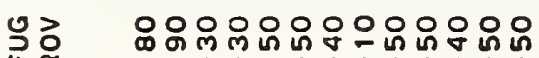

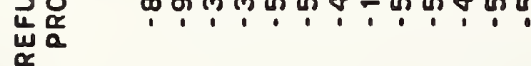

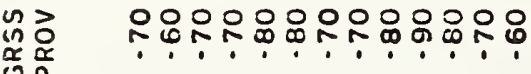

ơ

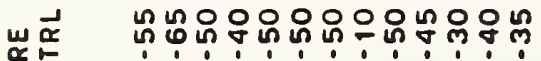

聯学

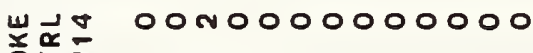
인든

tzm 000 No00000000

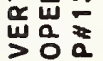

รูกับ ü a

מת

खण्व

צח

究言这

요 $0000000 \times 0000 \%$

岁枵a

นñ $00000000-0000$

ต잉

는

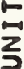

舀に

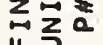

站㔯 No00000000NOO 운웅

山Uㅇ ONO00000000NO 는

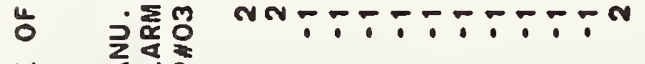
岁 这远a $\sum_{i}^{5}$ a

ज

$\$ 800000000000$

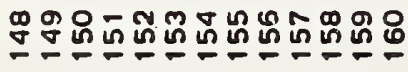




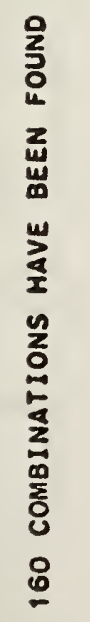


$\sum_{\substack{\alpha \\ 0}} \frac{u}{\omega}$

웡

n?

๙

$\underset{\alpha}{\alpha} \frac{d}{\alpha}$

岁市

tzm

un a

I $N$

$\sum_{0}^{n} \alpha$

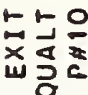

$\alpha \backsim g$

는은

un o

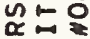

w

エ ル

ज正。

$4 z$

$\sum_{0}^{2} 0$

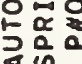

แ้ง

은

$j \stackrel{2}{2}$

$\alpha \backsim \boldsymbol{\gamma}$

눙

뜽

능

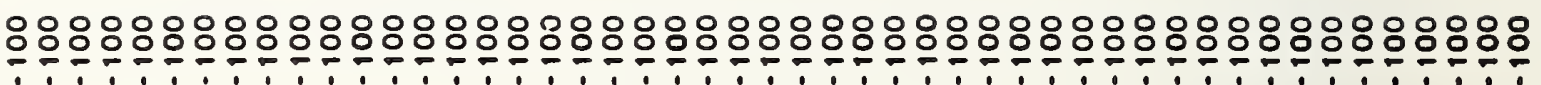

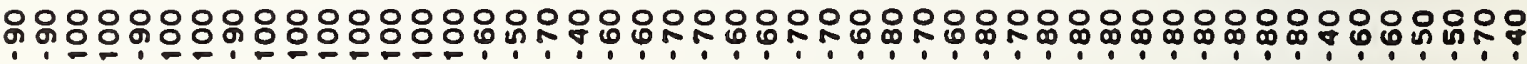

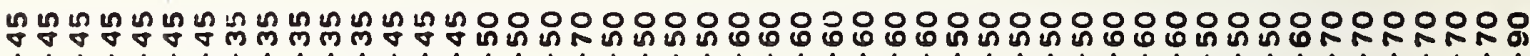

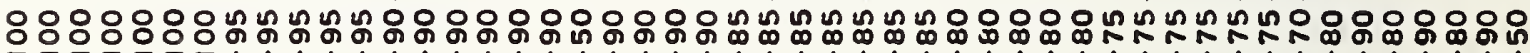

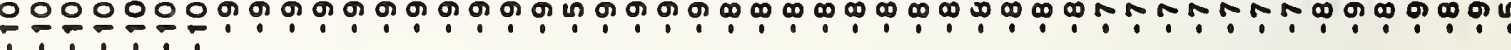

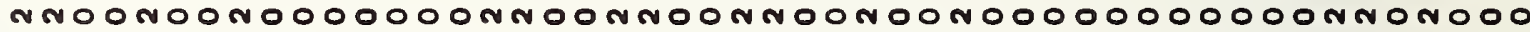
0000000000000000000000000000000000000000000000000

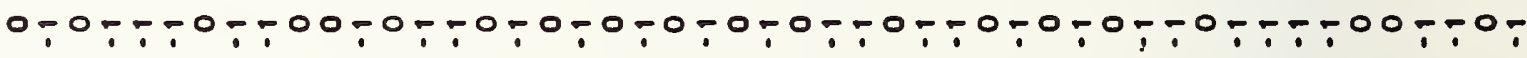

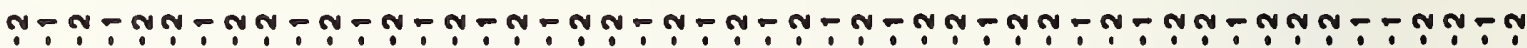
4. กง $0000--000-500-0000---0000--0000-00-00-00000000$

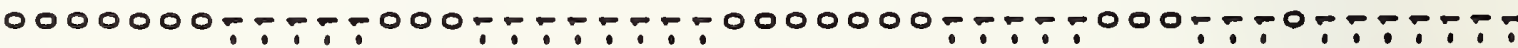

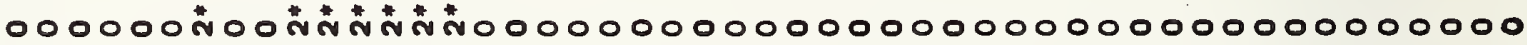

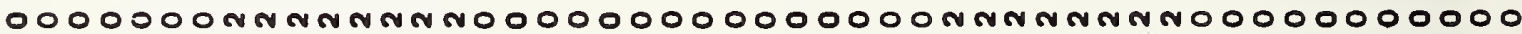

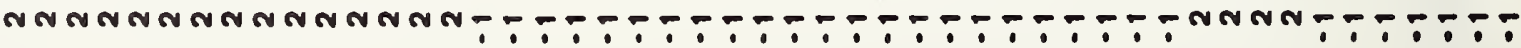
若

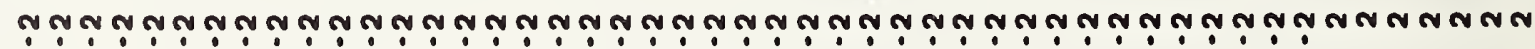
n

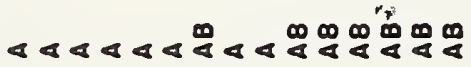

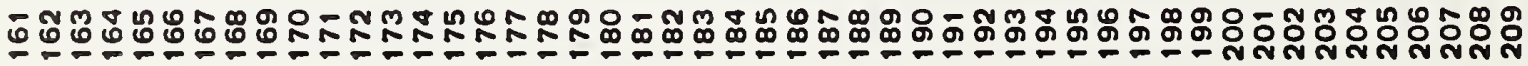


离嵌

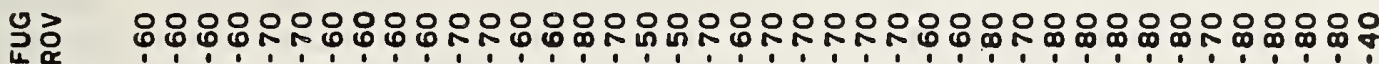
$\underset{\alpha}{\alpha}$

$n \geq$
$w$
$\alpha$
0

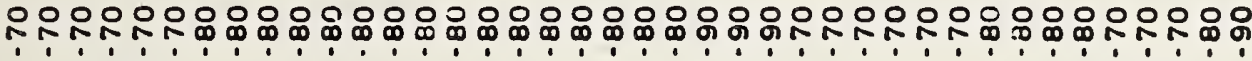

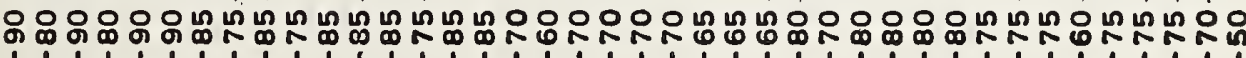

$\stackrel{-1}{u}$

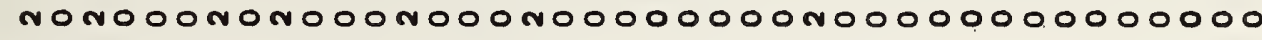

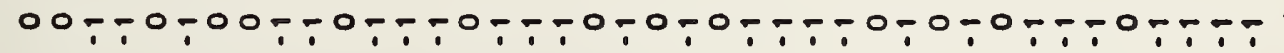

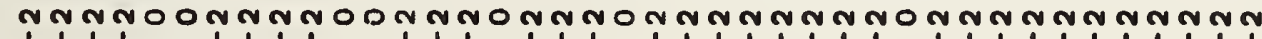

$\varphi \varphi \varphi \varphi \varphi \varphi \varphi \varphi \varphi \varphi \varphi \varphi \varphi \varphi \varphi \varphi \varphi \varphi \varphi \varphi \varphi \varphi \varphi \varphi \varphi \varphi \varphi \varphi \varphi \varphi \varphi \varphi \varphi \varphi \varphi \varphi \varphi \varphi \varphi \varphi$

- - - - $000000---0000--00-0000--00=000=00$

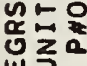

In

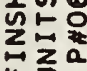

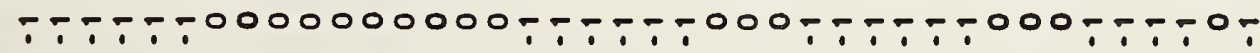

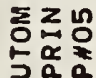

0000000000000000 nNm nNm nNo 山U大

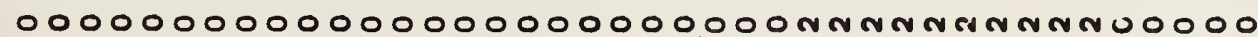

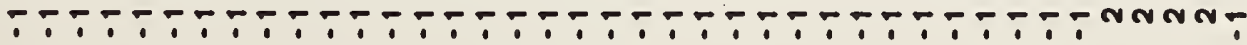

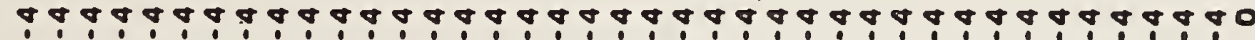

노웅

동

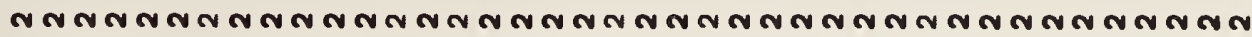

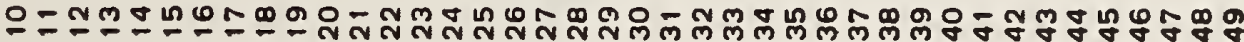

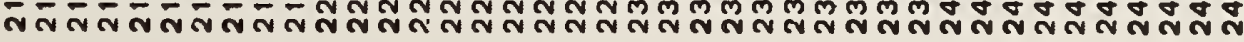




\section{APPENDIX D}

Instructional Manual

This manual is provided to assist in completing the Fire Safety Evaluation Worksheets for multifamily residences. The step-by-step instructions for the mechanisms of completing the worksheet are included in the worksheet itself. They are not repeated in this manual. This manual provides expanded discussion and definitions for the various items in the worksheet to assist the user when questions of definition or interpretation arise. To the maximum extent possible, the manual does not repeat the definitions already existing in the Minimum Property Standards (MPS) but rather references the appropriate MPS section. This manual is organized to progressively follow the format of the worksheet.

Contents

Page

Area of Application ................ . . . D2

Maintenance ................ . . . D2

Safety Parameters (General Discussion) . . . . . . . . D2

Construction .................... D2

Hazardous Areas .. . . . . . . . . . . . D3

Manual Fire Alarm ................. D5

Smoke Protection \& Alarm . . . . . . . . . . . D5

Automatic Sprinklers ................ D7

Interior Finish (Within Living limits) . . . . . . . . D7

Living Units Bedroom Doors ............... D8

Egress from Living Units ................. D9

Separation of Living Units from Each Other ......... D9

Exit System....................... D11

Exit Access . . . . . . . . . . . . . . . D13

Interior Finish (Egress Routes) . . . . . . . . . D13

Vertical Openings .................. D14

Smoke Control ..................... D14

Figure D-1 Construction Classification . . . . . . . . D17

Figure D-2 Hazardous Area - Level of Hazard . . . . . . . D18

Figure D-3 Hazardous Areas - Degree of Deficiency . . . . . D19 
Area of Application

The entire apartment building is evaluated on a single worksheet with the following options:

a. Spaces that are not used for living units, in direct utility or maintenance support of the living units, provided for tenant use, or in any way involved in tenant emergency egress may be omitted from the calculation when such space is separated from all of the tenant and tenant support spaces by two hour fire resistive construction (including any members that bear the load of tenant use space and with fire doors conforming to the requirements of section 4056.5 in any communicating opening). In such case, however, any appropriate charges under Item 2, Hazardous Areas in Table 1 shall be charged.

b. Where an apartment building is divided into two or more elements by firewall(s) meeting the relevant requirements of sections 405-4 (Fire Resistance Requirements), 405-6.5 (Door Opening Ratings), and 405-7.1 (Firewalls, and Lot-Line Wal1s) the facility may be rated as separate buildings or as a single building whichever gives the better (higher) rating.

\section{Maintenance}

Any protection system, requirements, arrangements, or procedures which are not maintained in a dependable operating condition, are used in such a manner that the intended fire safety function or hazard constraint is impaired or are not in a sufficient state of readiness should be considered as defective and receive no credit in the evaluation.

Safety Parameter Table (General Discussion)

The safety parameters are a measure of those building factors that bear upon or contribute to the safety of those persons who may be in the building at the time of a fire.

Fach of the safety parameters is to be analyzed, and the safety value for each parameter that best describes the condition in the building is to be identified. Only one value for each of the parameters is to be chosen. If two or more appear to apply, the one with the lowest point value governs.

\section{Construction}

Construction types are defined by the fire resistance and combustibility of load-bearing framing members, floor construction, and roof construction. In general, the requirements match those for the same members in section 405-4 and Table 4-5.1 of the MPS. Several additional categories however, have been added to provide a means of evaluating the impact of construction systems that include 
members or assemblies less fire resistant than the minimum listed for any of the types of construction shown in Table 4-5.1 (i.e. Unprotected structural systems). These have been designated types 2-U, 3-U, and 4-U. Figure 1 provides the appropriate data for classification of construction type in a tabular form similar to Table 4-5.1 of MPS. Figure 1, however, covers only the items necessary for classification of construction types recognized in the evaluation system.

The requirements for the elements in Table 4-5.1 that are not included under construction types are included under a variety of other parameters.

Where the building includes additions or connected structures of different construction, the rating and classification of the building is to be based on (a) separate buildings if a two hour or greater fire resistive separation exists between the portions of the building and on (b) the lower safety parameter's point score involved if such separation does not exist.

The story used to determine the parameter value is the highest story used for sleeping purposes. Story height is based on stories starting with the grade floor or the lowest floor used for sleeping purposes, whichever indicates the greater number of floors.

Any building involving conditions that fail to meet the requirements of section 405-7.2b for firestopping of concealed floor and roof spaces of combustible construction is to be classified as an unprotected type of construction (i.e., 4-U, 3-U, or 2-U as otherwise appropriate).

\section{Hazard Areas}

The assignment of charges for hazardous areas is a four-step process.

Step 1. Identify Hazardous Areas. A hazardous area is any space or compartment that contains a storage or other activity that is not a part of normal household living space arrangements and possesses the potential of producing a fully involved fire. A list of typical hazardous areas is listed under the heading, Exposure, in Figure 2.

Step 2. Determine the Level of Hazard. There are two levels of hazard as follows:

a. Structurally Endangering. A hazardous occupancy with sufficient fire or explosion potential to defeat the basic integrity of all exposed elements of the building framing as defined in Parameter No. 1 .

b. Not Structurally Endangering. A hazardous occupancy with sufficient fire potential to build to full involvement and presents a danger of propagating through openings or wall 
partitions but not possessing sufficient total potential to endanger any exposed elements of the structural framing or floor decking as defined in Parameter No. 1.

NOTE: Where a hazardous area is exterior to the building (an exterior exposure) the fire resistance of the exposed portions of the building shall govern the classification as shown in Figure 2. Where an in-building hazardous area is enclosed within a portion of the building having fire resistance above that credited for the building in Parameter No. 1, the minimum fire resistance of bearing walls, bearing partitions, columns, beams, girders, trusses, and floor-ceiling assemblies exposed to the hazardous area shall be used in determining whether or not the area is structurally endangering.

Where the vertical separation of exterior openings does not meet the requirements of Section 405-10.2, the facility shall be charged as having "Not Structurally Endangering" hazardous areas "Outside Living Units".

Figure 2 provides an analysis of typical types of hazardous areas relative to inherent potential structural danger to different classes of structural systems.

Step 3. Determine the Fire Protection Provided. The parameter value for hazardous areas is based on the presence or absence of the fire protection necessary to control or confine the hazard. Two different types of fire protection are considered. Th first consists of automatic sprinklers or other appropriate extinguishing system covering the entire hazard.* The second is a complete fire resistive enclosure including any bearing members in the space, partitions separating the hazardous area from all other spaces, and doors to the space sufficient to exceed the potential of the fire load involved. Any hazardous space that has either protection system is classified as having single protection. Any hazardous space that is both fully enclosed in a capable fire resistive enclosure and sprinklered is classified as having both (i.e. double level protection). On this basis, any fuel load that has the potential of overwhelming the available structural capability could as a maximum have single protection.

* When the hazardous area is within a living unit or abuts an egress route (exit access or exit) addressed in Parameters 10, 11 , or 12, the credit for sprinklers is not to be given unless the hazardous area is separated from the rest of the living unit or the egress route by reasonably smoke resisting partitions and doors.

Step 4. Determine Degree of Deficiency and Assign Parameter Values. The parameter value is finally determined on the basis of the degree of deficiencies that the hazardous area has in terms of the level of protection needed. 
Figure 3 provides a matrix type table to assist in determining degree of deficiency to be assessed.

In some situations, more than one hazardous area with the same or differing levels of deficiency will exist. The charge is based on the single most serious charge for hazardous area found.

\section{Manual Fire Alarm}

Manual fire alarm systems shall conform to the applicable requirements of NFPA 72A. The manual alarm system categories are as follows:

a. None. There is no manual fire alarm system, or the system is incomplete and does not meet the requirements necessary for a higher scored category.

b. W/O F.D. Notif. There is a manual fire alarm system with at least one manual fire alarm box per floor located in each credited path of egress and at least one sounding device per floor which is clearly audible in the sleeping areas of each apartment. Operation of any manual box shall cause the operation of all sounding devices. Buildings may have a zoned, non-coded alarm system which sounds the initial alarm only on the fire floor, and the floors directly above and below the fire floor with provision at a central monitoring point to activate a general fire alarm.

c. W/ F.D. Notif. There is a manual fire alarm system which complies with the requirements of $b$ above and, in addition, automatically transmits a signal to the fire department which is legally committed to serve the area in which the building is located, through a direct connection, an approved central station, or through other means acceptable to the authority having jurisdiction. No credit is given for F.D. Notif. in buildings higher than six stories if an annuciator which indicates the location of the fire by floor is not provided.

\section{Smoke Detection and Alarm}

A detection system as used herein is one based on the use of smoke detectors. No recognition is given for thermal detectors. The detection system categories are as follows:

a. None. There are no smoke detectors in the building or if any are. present they do not meet the requirements necessary for a higher scored category. 
b. Single Station Detectors. Single station detectors are those detectors that sound the alarm only at the detector itself. The operation of single station units does not involve the transmission of the alarm beyond the sounding of the alarm device in the unit itself.

(1) Single Level (Sin. Lev.). This value applies only where the facility includes one or more multi-level living units that do not have one or more single station detectors on each level of the living unit but at least has one detector in the immediate vicinity of each separate sleeping area.

(2) Every Level (Ev. Lev.). This parameter applies to those situations where there is one detector in each single leve1 living unit and one detector on each level of any multi-level living units.

c. Interconnected Systems. Interconnected systems are those systems so arranged that the operation of any detector within the living unit sounds alarm devices on other detectors or other separate alarm systems sufficient to alert all of the occupants. Where the systems are of the total building variety, or where the parameter value for Parameter 9, Separation of Living Units is a negative value, the credit can be given only if the building has a manual fire alarm system and the operation of the detection system sounds the manual fire alarm as though a fire alarm box on that floor had been operated. Interconnected systems for living units must provide sounding devices that are sufficient in location and loudness to assure the awakening of normally sleeping persons.

(1) Every Level (Ev. Lev.). This parameter applies to those situations where there is one detector in each single level living unit and one detector on each level of any multi-level living units.

(2) Plus Every Bedroom ( + Ev. Bedroom). To be credited for every bedroom a single level living unit must have a smoke detector in every bedroom and at least one additional detector in the common living space not involved in the bedroom. In a multi-level living unit there must also be at least one detector on each level in addition to the preceding requirement. 
(3) Total Building Systems. This system includes detector locations meeting the every bedroom standard throughout each living unit and also provides detector coverage throughout all corridors, common spaces, and hazardous areas with the system either incorporated within the manual fire alarm system or meeting the requirements for an automatic fire alarm system in accords with NFPA Standard No. 72 .

\section{Automatic Sprinklers}

a. None. No credit is given if there are no sprinklers or if sprinklers, though present are not sufficient to qualify for one of the other categories listed herein.

Note: Any space that is to be credited as being protected by automatic sprinklers that abuts a hazardous area that is deficient in accordance with Parameter 2, Hazardous Areas, will not be considered as sprinkler protected unless that hazardous area is also sprinkler protected.

b. Corridors and Public Spaces. Sprinkler protection covers all of the corridors and public spaces that separate, directly expose, or are in the egress path from the living units (except fire resistive enclosed non-combustible stairwells).

c. Living Units Only. All living units have sprinkler protection complying with the requirements for light hazard protection in NFPA Standard No. 13.

d. Corridors and Public Habitable Spaces. Meets the combined requirements for $b$ and $c$, above.

e. Total Building. The building is totally sprinkler protected in accordance with NFPA Standard No. 13 for light hazard occupancy (or higher hazard occupancy for any spaces classified as higher hazard by NFPA Standard No. 13) and is equipped with an automatic alarm initiating device that will activate the the building manual fire alarm system as credited in Item 3, Manual Fire Alarm.

6. Interior Finish (Within Living Units)

The interior finish within the living units is evaluated separately from the interior finish in the corridor and egress routes and other public space. The types of surfaces covered, exemptions and flame spread tests, are as defined in Section 405-8. 
No consideration is given for any interior finish with a flame spread rating $>200$ or for any material not rationally measured by the ASTM E84 Test. Materials not rationally measured include: foam plastics, asphalt impregnated paper and/or materials capable of inducing extreme rates of fire growth and rapid flashover. In any case where these materials are involved the resultant risk is considered beyond the capacity of this evaluation system and will require individual appraisal.

In assessing the charge for interior finish, use the most combustible interior finish as defined by Section 405-8.

NOTE: $1 / 4$ inch plywood can be considered as having a flame spread of 200 or less.

\section{Living Unit Bedroom Doors}

a. No Door. The charge for no door shall be assessed if any bedroom or other room used for sleeping purposes does not have a door; has a door but there is some mechanism or obstruction which prevents closing of the door or otherwise leaves a significant opening between the door and the corridor; the door has open louvers; or has ordinary glass lights or transoms.* Doors that have been blocked open by doorstops, chocks, tiebacks, "or other devices that require manual unlatching or releasing action to close the door shall be classified as "No Door". Also doors that are not provided with a latch or other device suitable for keeping the door tightly closed shall be classified as "No Door".

* Ordinary glass lights shall not be considered as requiring the "No Door" classification in locations where both sides of the glass light are protected by automatic sprinklers.

b. Doors With Less Than 20 Minute Fire Resistance ( $<20$ Min.). Doors which are not deficient as described in a, but do not meet the requirements for $\mathrm{c}$, below, will be classified as less than 20 minute fire resistance.

c. 20 Minute or More Fire Resistance. Doors shall be considered as having 20 minute or greater fire resistance if they are $1-3 / 4$ inch solid core wood construction or any other arrangement of equal or greater stability in fire integrity. The thermal insulation capability of the door is not considered. Hollow or sheet steel doors therefore sha11 be considered to meet the 20 minute requirement. 
a. Single Route. The single route charge is assessed in any location where there is only one emergency exit route from either a single or multi-level unit with no alternatives meeting any of the requirements in the following subparagraphs. A living unit with two doors may still be charged with a single route if the two doors are in such proximity to each other in terms of the living unit that they do not provide alternative routes.

b. One Door Plus Escape Windows. This value is credited when the unit has a door providing a means of unobstructed travel out of the living unit and there are outside windows operable from the inside without the use of tools that provide a clear opening of not less than 20 inches in width, 24 inches in height and $5.7 \mathrm{sq}$. ft. in area. The bottom of the window shall not be more than 44 inches above the floor.

c. Remote Exits. Remote exits exist when there are two exit doorways from the living unit leading to direct or stairway access to the street so located that occupants of any bedrooms or sleeping areas have an option for two alternative directions of egress immediately upon leaving the bedrooms or sleeping area.

d. Greater Than 50 Foot Travel (>50 Ft. Travel). The charge for $>50 \mathrm{ft}$. travel is to be assessed if any living units assessed as either "Single Route" (a, above) or "One Door Plus Escape Windows" ( $b$, above) if the distance within a living unit between the door to the most remote room on a doorway to an exit or exit corridor exceeds 50 feet.

\section{Separation of Living Units from Each Other and from Common Spaces}

Separation of living units from each other and from common spaces shall be based on the wall partition making the separation and the protection of the openings in those partitions. Ceiling and floor partitioning will not be considered in this category. These elements are covered under Parameter No. 1, Construction.

Duct penetrations where the duct is open on one side only of the partition and is of sheet steel construction shall be considered as equivalent to doors having a fire resistance of at least 20 minutes. Where there are duct openings on both sides of the partition the opening shall be considered unprotected unless there is a fire damper in the duct opening or the duct otherwise meets the requirements for omission of fire dampers as specified in NFPA Standard No. 90A. 
The partition shall be considered as "None or Incomplete" if it has unprotected openings (louvers, gaps, transfer grills, plain glass windows, or plain glass transoms) between the floor and the ceiling. If openings exist above the ceiling level (or even if the partition stops at the ceiling level), the walls shall be considered as complete if the ceiling itself is a complete membrane (such as plasterboard or lathe and plaster). In which case, the fire resistance rating shall be based on that of the wall or ceiling system whichever is less.

Walls shall be considered to have less than 20 minute ratings (Walls <20 Min) if they are not equivalent to $1 / 2$ inch gypsum wal1board on both sides of studs well nailed or fastened to the studs with appropriate taping and finishing of joints and fasteners. Walls shall be considered as equal to or greater than 1 hour (Walls $\geq 1 \mathrm{Hr}$ ) if they are of any of the established systems recognized as having 1 hour or greater fire resistance in accordance with recognized tests or approved listings.

Walls are considered to have fire resistance ratings of $1 \mathrm{Hr}$ or greater when they are of a construction demonstrated to have such resistance by ASTM E-119 or equivalent qualifications.

Doors shall be considered as "None or Incomplete" if any living unit does not have a door; has a door but there is some mechanism or obstruction which prevents closing of the door or otherwise leaves a significant opening between the door and the corridor; the door has open louvers; or has ordinary glass lights or transoms.* Doors that have been blocked open by doorstops, chalks, tiebacks, or other devices that require manual unlatching or releasing action to close the door shall be classified as "None or Incomplete". A1so doors that are not provided with a latch or other device suitable for keeping the door tightly closed shall be classified as "No Door".

* Ordinary glass lights shall not be considered as requiring the "No Door" classification in locations where both sides of the glass light are protected by automatic sprinklers.

The separation shall be considered as "None or Incomplete" if either the partitions or doors involved are so classed.

Doors shall be considered as having 20 minute or greater fire resistance (Doors $\geq$ Min) if they are a 1-3/4 inch thick solid core wood construction or arrangement of equal or greater stability in fire integrity. The thermal insulation capability of the door is not considered. Hollow or sheet steel doors therefore meet the 20 minute requirement. 
Doors are to be considered as having 45 minute or greater fire resistance if they are so listed or labeled by an acceptable testing laboratory or otherwise shown to meet the requirements for such resistance in accordance with NFPA Standard No. 80.

Automatic closing doors (W/A.C.) shall be considered automatic closing if they are provided with devices that either provide the traditional self-closing mechanisms or a smoke detector closing mechanism. In the case of doors separating living units from each other or from common spaces, self-closing doors shall be accepted whether or not they are equipped with devices that can be used to hold them in the open position so long as the normal operating mode of the living unit is to keep the door closed, particularly after the occupants have retired for the night.

NOTE: This parameter does not cover the charges for dead end conditions, travel distance, interior finish in the exits or exit access routes, or enclosure of stairways or other exit routes that pass from floor to floor. These elements are separately covered in Parameters 11, Exit Access; 12, Interior Finish (Egress Routes); and 13, Vertical Openings.

\section{Exit System}

Exit routes are the paths of travel from the living unit to the outside of any of the types and arrangements described in Sections 402-1 through 402-8 of the Minimum Property Standards.

Where subdivision of concealed floor and roof spaces of combustible construction fails to meet the criteria of 405-7.2b, the evaluation is based on the resultant conditions with the maximum credit based on less than 1 hour walls $(<1 \mathrm{Hr}$ ).

a. Multiple Routes. Multiple routes exist when the occupants of any living unit have either from the living unit or through access in a corridor adjacent to the living unit a choice of 2 separate exit routes to the outside of the types described in Sections 402-2 through 402-7, 403 or 405-6.

b. Deficient. An exit route is deficient if it fails to meet any of the applicable criteria in Sections 402, or 405-6, of the Minimum Property Standards except for those covered in Parameters 11,12 , and 13 . The exit system is also graded as deficient if there is not at least one horizontal exit on each floor of any building that is graded $2 \mathrm{U}, 3 \mathrm{U}$, or $4 \mathrm{U}$ construction or that exceeds the area limitations in Table 4-5.2. 
c. Without Horizontal Exits (W/O Horiz.). Egress system is based on this charge if there are multiple routes that are not deficient but the arrangement does not include a horizontal exit as defined below or have acceptable direct exit from each living unit as defined below.

(1) In addition this category shall apply to special cases meeting the following:

(a) A one story building containing a maximum of 8 living units.

(b) A 2 story building containing a maximum of 8 living units and not more than 4 units per floor with one hr. fire resistive enclosed stairway immediately accessible to all living units.

(c) A 3 or 4 story building having not more than 4 living units per floor with a smokeproof tower, or a fire resistive enclosed stairway with a $2 \mathrm{hr}$. rating for a four-story building and a one hr. rating for a three-story building immediately accessible to all living units.

d. Horizontal Exit (W/ Horiz. Exit). The credit for horizontal exit is to be given if each floor containing living units has at least one horizontal exit meeting all of the following:

(1) The horizontal exit meets the definition of such in Appendix A of the MPS.

(2) Each refuge area formed by a horizontal exit has access to at least one exit from the building without recrossing the line of the horizontal exits.

(3) The space in each refuge area formed by a horizontal exit provides at least $3 \mathrm{sq}$. feet of accessible space for all of the potential occupants already present in or evacuating to it.

e. Direct Exits. To be credited with direct exits each living unit shall have within that unit a door that opens to the exterior at grade or onto an exterior balcony with direct access to an exterior exit or smoke proof tower. Where such openings are directly onto grade in a location where any person egressing can move directly away from the building without further exposure the credit for direct exit is applicable even if there are no other exit routes from the involved living unit. (The assessment of charges for Parameter 8, Egress from Living Units is not altered by this allowance.) 
11. Exit Access

Exit access is a measurement of the travel from the living unit to the outside or to an enclosed interior stairway or other exit (e.g. horizontal exit) or smoke partition as defined in Parameter 14, whichever is shorter.

The charge for dead-end (D.E.) access shall be charged when any corridor affords access in only one direction to a required exit from that corridor. The calculation of the distance to determine the level of charge is the measurement from the centerline of the doorway exiting the most remote living unit to the point offering two or more directions of exit. Exit travel is the distance from the door of the living unit to the point where the building is exited or a stairwell is entered whichever is less. Where the distance to the stairwell is shorter that distance shall be based on the distance to the door enclosing the stairwell if the stairwell is enclosed or to the top tread if the stairwell is open.

\section{Interior Finish (Corridors and Egress Routes)}

The interior finish within the living units is evaluated separately from the interior finish in the corridor and egress routes and other public space. The types of surfaces covered, exemptions and flame spread tests, are as defined in section 405-8.

No consideration is given for any interior finish with a flame spread rating $>200$ or for any material not rationally measured by the ASTM E84 Test. Materials not rationally measured include: foam plastics, asphalt impregnated paper and/or materials capable of inducing extreme rates of fire growth and rapid flashover. In any case where these materials are involved the resultant risk is considered beyond the capacity of this evaluation system and will require individual appraisal.

In assessing the charge for interior finish, use the most combustible interior finish as defined in Section 405-8.

NOTE: $1 / 4$ inch plywood can be considered as having a flame spread of 200 or less.

The corridors and egress route, for purposes of this parameter, is the complete system-corridors, stairs, lobbies and any other spaces open to the primary or alternate routes of exit from every living unit to the exterior. A space shall be considered open to the exit route if it is not a living unit and is not separated by interior partitions with self-closing door having fire resisting capabilities equal to that credited by item 9, or item 13, whichever is greater. 
13. Vertical Openings

These values apply to vertical openings and penetrations including exit stairways, ramps and any other vertical exits, pipeshafts ventilation shafts, duct penetrations and laundry and incinerator chutes. The charge for vertical openings shall be based on the presence or lack of enclosure and the fire resistance of enclosure if present.

A vertical opening or penetration shall be classified as open (or Incomplete Enclosure) if it is: (a) unenclosed; (b) is enclosed but does not have doors; (c) is enclosed but has openings other than doorways; (d) is enclosed with cloth, paper or similar materials without any sustained fire stopping capabilities.

If a shaft other than in a credited exit route (i.e. credited as one of the multiple routes required in Parameter 10 or in determining travel distance in Parameter 11) is enclosed on all floors but one and this results in an unprotected opening between that shaft, and one and only one floor, the parameter value assigned to that shaft shall be 0 . If a required egress route is contained in that shaft the parameter value shall be -2 .

If firestopping fails to conform with the requirements of 405-7.2a, the vertical opening so created shall be evaluated using the above criteria.

\section{Smoke Control}

Smoke control definitions are as follows:

a. No Control. There are no smoke barriers (or horizontal exits) on the floor, the floor is not served by a smoke proof stairtower, and there are no mechanically assisted smoke control systems serving the floor.

b. Smoke Partitions. Smoke partitions consist of one-hour fire rated partitions extending across the entire width of the building or so arranged as to combine a partition in the corridor with existing building elements and subdividing partitions and walls to effectively completely partition the building into two separate units. The smoke partition must be equipped with 3/4-hour fire doors in the corridor that are closed upon detection by smoke detectors located at the door arches or by smoke detector systems that have been credited as the 6 point value in Parameter 4, Smoke Detection and Alarm. Each smoke compartment must also be serviced by an elevator as described in Section 405-9. Horizontal exits will act as a smoke partition and when it exists it is credited as both a smoke partition in Item 14 and a horizontal exit in Item 10 . 
c. Mechanically Assisted Systems - By Zone. Mechanically assisted smoke control on a zone basis must include a smoke partition (or a horizontal exit) supported by a mechanism of automatic control fans, smoke vent shafts, or a combination thereof to provide a pressure differential that will assist in confining smoke to the compartment of origin. Fans involved may be special smoke control fans or special adjustments of the normal building air movement fans.

d. Mechanically Assisted Systems - By Unit. Mechanically assisted smoke control on a living unit basis are systems so designed as to provide a mechanism of automatically controlled fans, smoke vent shafts, or combination thereof to insure a positive pressure differential that will prevent intrusion of smoke into any living unit not involved in fire. On this basis, the living unit will have a pressure differential higher than the corridor and higher than any living unit where fire has been detected. Such systems must be so arranged that there is detection in each living unit that will prevent a living unit that is involved in fire from becoming positively pressurized.

e. Mechanically Assisted Systems - Corridors. Mechanically assisted smoke control on a corridor basis is a system initiated by a method of smoke detection that will assure operation of the smoke control system before significant smoke has entered into the corridor involved. The mechanism must be capable of pressurizing the corridor sufficiently to prevent smoke from the living unit or space of origin from entering the corridor through the entire course of fire. Such a system must be able to hold back the smoke through the expected maximum severity of the fire. It must also be capable of evacuating smoke from the corridor on the presumption that the emergency evacuation procedures and other activities involving the opening and closing of doors will cause occasional brief periods of overpowering the smoke control system and movement of the smoke into the fire area of the corridor. (This would normally be accomplished by having an exhaust fan from the corridor of lower capacity than the fan supplying air for pressurization. The net pressurization force would occur from the effect of the pressurizing fan minus the effect of the removal or purging fan.) To be effective the corridor's pressurizer system would normally necessitate early warning smoke detection, automatic closing of all living unit doors, and/or sprinklered protection. Where these additional protection devices are provided in order to effect such a smoke control system the individual credits for each of the involved protection devices are in addition to the credits for the smoke control system. 
f. Smoke Proof Towers. Credit for smoke proof towers may be given if either the stairways meet the requirements for a smoke proof tower specified in Section 5-2.3 of the Life Safety Code (NFPA 101-1976) or has an acceptably designed smoke pressurization system maintaining a positive pressure in the stairwell sufficient to prevent intolerable contamination of the stairwell by smoke or other fire effects. 


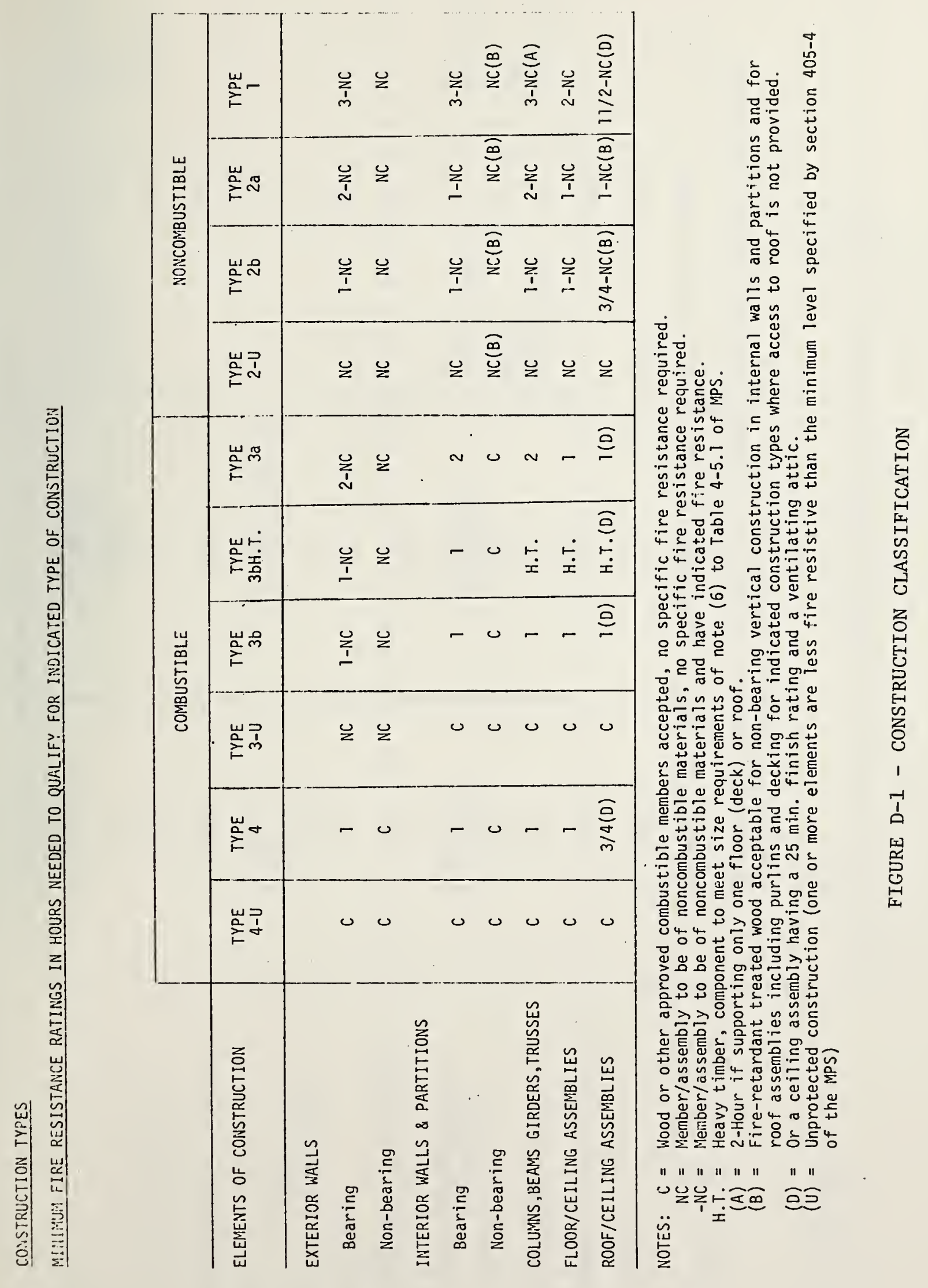



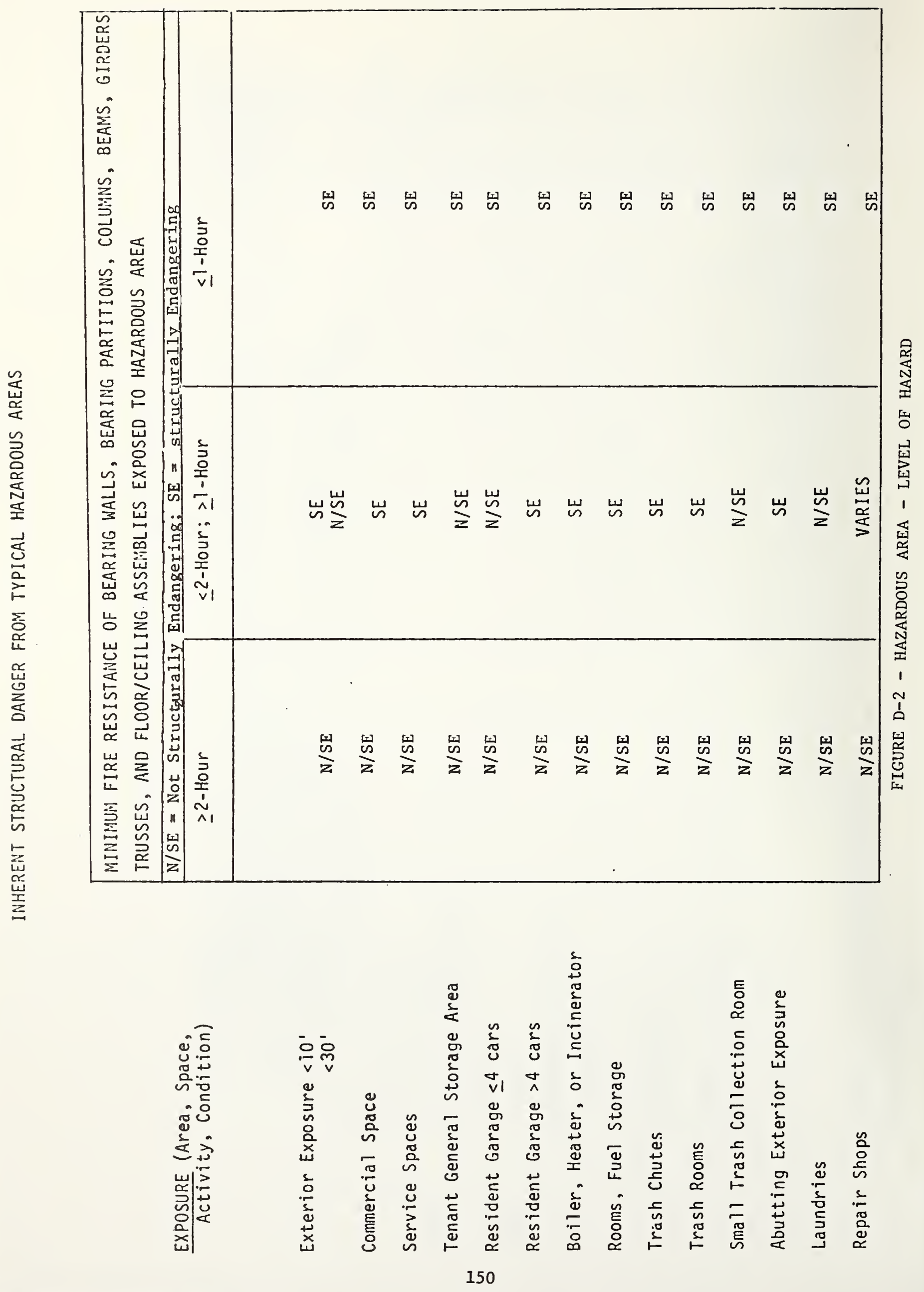


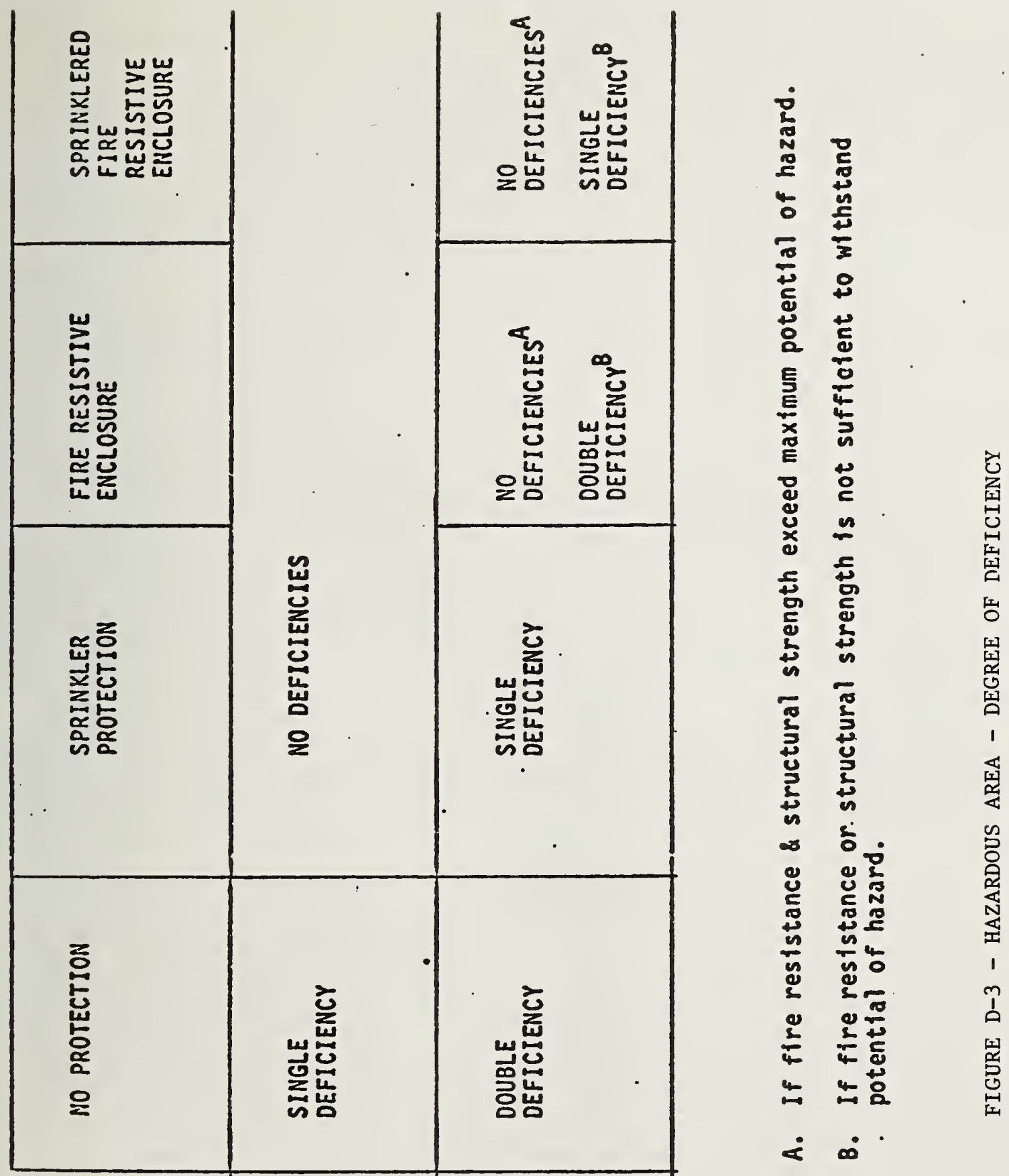


NBS-1 I4A (REV, 2-BC)

\begin{tabular}{|c|c|c|c|}
\hline $\begin{array}{l}\text { U.S. DEPT. OF COMM. } \\
\text { BIBLIOGRAPHIC DATA } \\
\text { SHEET (See instructions) }\end{array}$ & $\begin{array}{l}\text { 1. PUBLICATION OR } \\
\text { REPORT NO. } \\
\text { NBSIR } 82-2562\end{array}$ & 2. Performing Organ. Report No. & $\begin{array}{l}\text { 3. Publication Date } \\
\text { September } 1982\end{array}$ \\
\hline
\end{tabular}

4. TITLE AND SUBTITLE

A System for Fire Safety Evaluation for Multifamily Housing

5. $\operatorname{AUTHOR}(\mathrm{S})$

H.E. Nelson and A.J. Shibe

6. PERFORMING ORGANIZATION (If joint or other than NBS, see instructions)

7. Contract/Grant No.

NATIONAL BUREAU OF STANDARDS

DEPARTMENT OF COMMERCE

WASHINGTON, D.C. 20234

8. Type of Report \& Period Covered

Interim

9. SPCNSCRHIG ORGAHIZATION NAME AND COMPLETE ADDRESS (Street, City. Stote, ZIP)

Department of Housing and Urban Development

Washington, D.C. 20410

10. SUPPLEMENTARY NOTES

Document describes a computer program; SF-185, FIPS Software Summary, is attached.

11. ABSTRACT (A 200-word or less factual summary of most significant information. If document includes a significant bibliography or literature survey. mention it here)

A qualitative evaluation system for grading multifamily housing in terms of fire safety has been developed and is ready for testing in Department of Housing and Urban Development field offices. The system is designed to be used to identify a variety of combinations of widely accepted fire safety equipment and building construction features that provide a level of safety equal or greater than that achieved by conformance to the explicit requirements of the HUD Minimum Property Standards. In this evaluation, equivalent safety performance is gauged in terms of overall level of safety provided rather than a component by component comparison.

12. KEY WORDS (Six to twelve entries; alphabetical order; capitalize only proper names; and separate key words by semicolons) Building codes; building construction; Delphi Method; fire safety; interior finishes; Life Safety Code; Minimum Property Standards; multifamily housing; risk analysis; safety equivalency; safety evaluation; smoke detection; sprinkler systems.

13. AVAILABILITY

$\mathrm{x}$ Unlimited

For Official Distribution. Do Not Release to NTIS

Order From Superintendent of Documents, U.S. Government Printing Office, Washington, D.C. 20402.

14. NO. OF

PRINTED PAGES

$$
159
$$

15. Price

$\$ 15.00$

$\mathrm{X}$ Order From National Technical Information Service (NTIS), Springfield, VA. 2216I 

